\title{
Combining LC-MS/MS and genetic analysis to unravel plant hormone metabolism in Arabidopsis thaliana
}

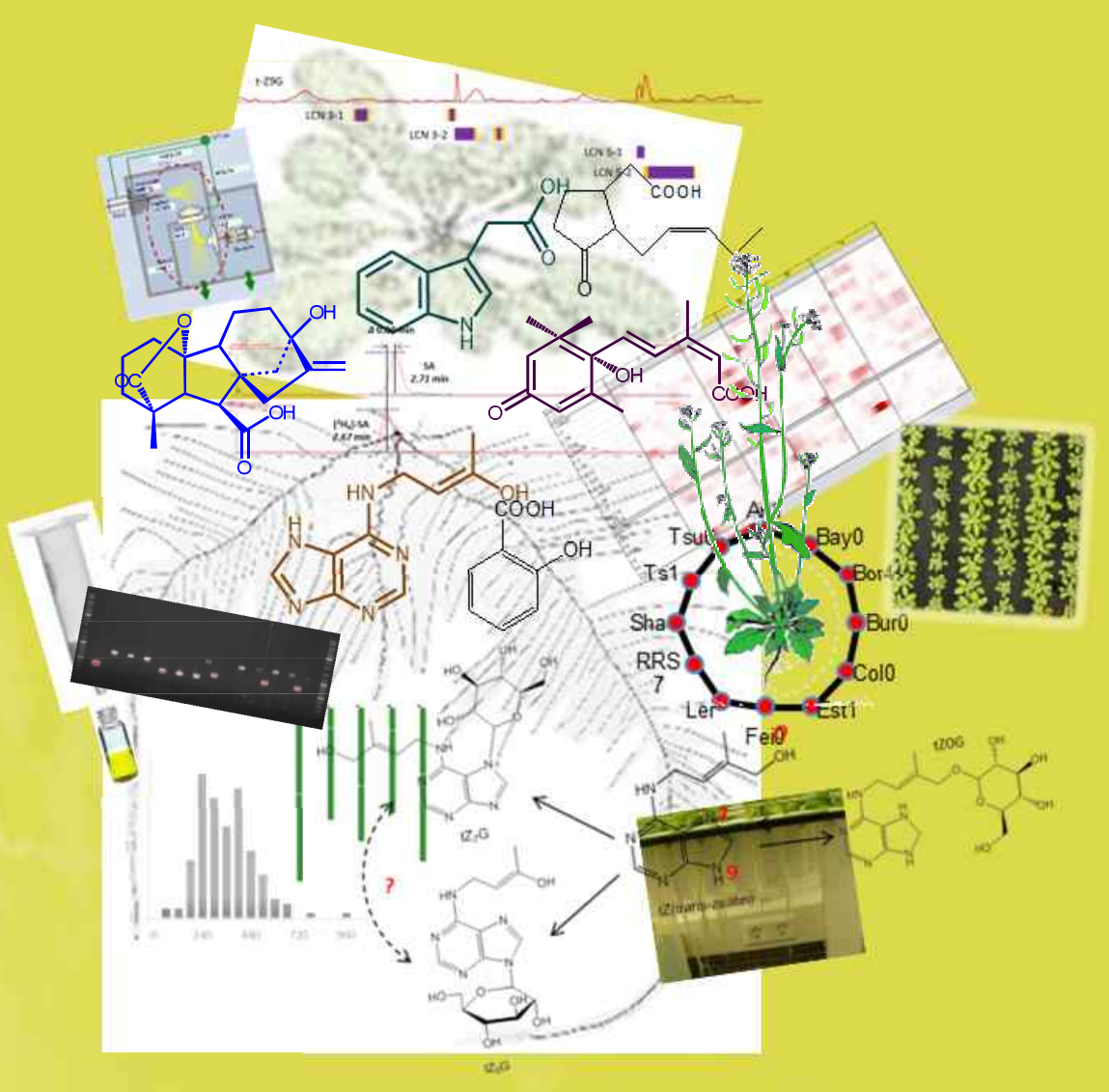

Sangseok Lee 


\section{Propositions}

1. Solving matrix effects is crucial for generating reliable data in quantitative analysis on LC-MS.

(this thesis)

2. Detection of QTLs for ratios between different groups of hormones may reflect complex molecular mechanisms underlying hormone crosstalk. (this thesis)

3. Map studies for interactome networks, such as done by the Arabidopsis Interactome Mapping Consortium (2011, Science 333, 601-607), provide splendid graphics but ambiguous interpretation.

4. The term 'evolutionary' in relation to natural variation incorrectly implies that this variation explains species diversity.

5. An individual's political disposition depends on which media he/she is exposed to.

6. The reason that organic farming in South Korea contributes only about $1 \%$ to agricultural food production is that it is not profitable.

Propositions belonging to the thesis, entitled

Combining LC-MS/MS and genetic analysis to unravel plant hormone metabolism in Arabidopsis thaliana

Sangseok Lee

Wageningen, 21 January 2020 
Combining LC-MS/MS and genetic analysis to unravel plant hormone metabolism in Arabidopsis thaliana 


\section{Thesis committee}

\section{Promotor}

Prof. Dr Harro J. M. Bouwmeester

Professor of Plant Physiology

Wageningen University \& Research

Chair of Plant Hormone Biology

University of Amsterdam

\section{Co-promotors}

Dr Dick Vreugdenhil

Associate professor, Laboratory of Plant Physiology

Wageningen University \& Research

Dr Lidiya I. Sergeeva

Laboratory of Plant Physiology

Wageningen University \& Research

\section{Other members}

Prof. Joost Keurentjes, Wageningen University \& Research

Prof. Michel Haring, University of Amsterdam

Dr Ivo Rieu, Radboud University, Nijmegen

Dr Gerard van der Linden, Wageningen University \& Research

This research was conducted under the auspices of the Graduate School of Experimental Plant Sciences (EPS). 


\title{
Combining LC-MS/MS and genetic analysis to unravel plant hormone metabolism in Arabidopsis thaliana
}

\author{
Sangseok Lee
}

\author{
Thesis \\ submitted in fulfilment of the requirements for the degree of doctor \\ at Wageningen University \\ by the authority of the Rector Magnificus, \\ Prof. Dr A.P.J. Mol, \\ in the presence of the \\ Thesis Committee appointed by the Academic Board \\ to be defended in public \\ on Tuesday 21 January 2020 \\ at 1:30 p.m. in the Aula
}


Sangseok Lee

Combining LC-MS/MS and genetic analysis to unravel plant hormone metabolism in Arabidopsis thaliana

142 pages,

$\mathrm{PhD}$ thesis Wageningen University, Wageningen, the Netherlands (2020)

With references, with summary in English

ISBN: 978-94-6395-240-8

DOI: http://doi.org/10.18174/508861 


\section{CONTENTS}

Chapter 1

p. 7

General Introduction

Chapter 2

p. 17

Methodological validation: quantitative analysis of various classes of plant hormones in leaves and roots of Arabidopsis using UPLC-ESI-MS/MS

Chapter 3

p. 35

Natural variation of hormone levels in Arabidopsis roots and correlations with

complex root architecture

Chapter 4

p. 61

Quantitative trait loci analysis of hormone levels in Arabidopsis roots

Chapter 5

p. 87

Quantitative trait loci for hormone levels in Arabidopsis leaves

Chapter 6

p. 117

General Discussion

References

Summary

p. 138 

Chapter 1

General Introduction 
As sedentary organisms, plants are exposed to enormous environmental challenges during growth and development. In plants, environmental conditions are translated into cellular responses through internal signals, including plant hormones. The interplay between external cues and internal signals results in plant adaptation. Furthermore, plant hormones, alone or in interactions, play important roles in rapid adjustment of development via changes in their levels and/or changes in their perception. This thesis contains a number of studies on plant hormones: how quantitative levels of diverse plant hormones can be determined reliably; what extent of variation in endogenous hormones exists across Arabidopsis natural accessions; is it possible to detect quantitative trait loci for hormone levels using a set of recombinant inbred lines in Arabidopsis thaliana. The findings described in this thesis contribute to a better understanding of the genetic basis of the regulation of hormone levels in the experimental plant model, $A$. thaliana.

\section{Roles of plant hormones in growth and development}

Plant hormones are a group of organic substances present in low concentrations that mediate an array of physiological processes. In 1928, Went in the Netherlands first isolated a chemical compound from wheat coleoptiles, showed curvature of the coleoptile towards light a phenomenon called phototropism. This substance was originally named Wuchsstoff by Went, and later this was changed to indole-3-acetic acid (IAA), also known as auxin (Went and Thimann, 1937). Other investigations led to a series of discoveries of other hormones: studies in plant-pathogen interactions resulted in the finding of gibberellins (GAs); attempts to culture tissues in vitro led to the discovery of cytokinins (CKs); studies on dormancy and abscission led to the discovery of abscisic acid (ABA) (although abscission was later shown not to be regulated by ABA but by ethylene); the effects of gas used in streetlights on nearby trees led to the discovery of the gaseous hormone ethylene (ET) (Vanstraelen and Benkova, 2012).

Auxin is by far the most abundant and physiologically significant in plants. IAA moves mainly from the apical (shoot meristem) to the basal end (root tip), so called polar transport. Auxin stimulates the rate of cell elongation, although in roots it can also inhibit cell elongation at the higher concentrations. Phototropism and gravitropism are also closely linked to the action of auxin; both tropisms are mediated by the lateral redistribution of auxin.

It is recognized that $\mathrm{ABA}$ plays an important role in stomatal closure, seed maturation and dormancy. An increase in cytosolic calcium is responsible for ABA-induced stomatal closure. Desiccation tolerance is also acquired under ABA control. Seed dormancy is controlled by the $\mathrm{ABA} / \mathrm{GA}$ balance, in which DELLA family proteins are degraded under elevated ABA levels.

The GAs, a family of compounds based on the ent-gibberellane structure, is known for its stem elongation stimulation. Among more than 125 chemical members of the GAs, $\mathrm{GA}_{1}$ and $\mathrm{GA}_{4}$ are the 
most important in plants as bioactive compound, with biological activities that differ depending on the plant species. GA promotes seed germination and stimulates growth of stem and root. Pollen development and male fertility are also regulated by bioactive GAs. One of the molecular mechanisms is the activation of $\alpha$-amylase gene expression by GA through a MYB transcription factor (Kaneko et al., 2004).

Although many previous studies revealed substances to be active for cell division, such as kinetin, trans-zeatin was the first cytokinin isolated from maize in the early 1960s. Together with auxin, CK is generally accepted to regulate the cell division cycle, and it has been known that the balance between these two hormones governs a large range of developmental aspects in plants. There are many naturally occurring CK metabolites, including sugar-conjugates and the basic outline of the metabolic pathways leading to these diverse CK species has been elucidated.

Jasmonic acid (JA) and salicylic acid (SA) play important roles in plant defence against insect feeding, and in resistance to pathogens involved in the systemic acquired resistance response (SAR), respectively. Other hormones, such as ET, brassinosteroids (BRs) and strigolactones, also play a significant role in plants but they are out of scope of this thesis, mainly because of limitations in their detection in complex matrices of plant extracts.

In recent years remarkable progress in our understanding of the plant hormone biosynthesis and signal transduction pathways have brought us a big step forward to understanding the complicated cross-talk (interactions) between hormones and how this is coordinated at the molecular level. The hormone-mediated plant response to environmental changes or challenges results from integrated signalling networks of multiple hormones, which are unlikely to be linked by linear pathways (Chandler, 2009). A large array of functional interactions exists among the individual hormones: e.g., auxin interacts with $\mathrm{CK}, \mathrm{ET}, \mathrm{GA}, \mathrm{ABA}, \mathrm{BR}, \mathrm{JA}$, and the recently discovered strigolactones (Davis, 2010; Moubayidin et al., 2009; Stirnberg et al., 2010; Swarup et al., 2002; Teale et al., 2008). For example, subtle spatial differences in the concentrations of auxin and CK determine root formation and its development (Bishopp et al., 2011). Such interconnectedness of plant hormone signalling pathways is also found in responses to environmental stresses. SA, JA, ABA and ET interact in the regulation of plant defence to biotic (pathogens and pests) and abiotic stresses (temperature and wounding) (Bari and Jones, 2009). Moreover, the signal-transduction pathways of different hormones show several similarities: e.g., the ubiquitin-proteasome pathway plays a key role in most hormonesignalling pathways (Nemhauser et al., 2006; Santner and Estelle, 2009). To shed more light on these cross-talk mechanisms underlying the regulation of plant responses by hormones, I decided to target a range of different hormones in a natural variation study and QTL analyses. 


\section{Significance of hormone analysis}

Plants synthesize a huge array of metabolites. Like many other metabolites, plant hormones display structural diversity possibly as a consequence of their homeostasis pathways. Within a certain hormone group, usually only a few are biologically active, such as $\mathrm{GA}_{1}, \mathrm{GA}_{3}$ and $\mathrm{GA}_{4}$, while many others are inactive in physiological processes. Now a wealth of information of hormone metabolism has become available for the classical groups of plant hormones. Common features found in hormone pathways are considerable redundancy and adaptive plasticity in the network of their pathways leading to homeostasis.

Enzymes in metabolic pathways play a pivotal role in chemical reactions and their feedback loops between substrates (precursors) and products, eventually maintaining homeostasis in a series of intermediates. However, metabolic flux (the flow of molecules through a metabolic pathway) may also be regulated by the metabolites themselves (Farre et al., 2014). Therefore, it is better to target a wide range of hormone metabolites rather than a single active compound, in order to find diverse regulatory genetic loci in plant hormone metabolism when genetic mapping analysis is applied.

\section{Figure 1. Plant hormones targeted in this PhD study}<smiles>O=C(O)Cc1c[nH]c2ccccc12</smiles>

$\operatorname{auxin}(\mathrm{IAA})$<smiles>C/C(=C\CNc1ncnc2nc[nH]c12)CO</smiles>

trans-zeatin (CK)

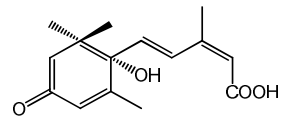

abscisic acid (ABA)<smiles>O=C(O)c1ccccc1O</smiles>

salicylic acid (SA)

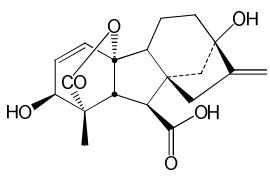

Gibberellic acid $\mathrm{A}_{3}(\mathrm{GA})$

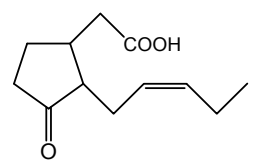

jasmonic acid (JA) 
The analysis of plant hormones is challenging due to their low concentrations. Most plant hormones are present in concentrations of $10^{-6} \mathrm{M}$ to $10^{-9} \mathrm{M}$ and are rapidly decomposed by light and enzymatic reactions unless samples are carefully kept in a controlled manner. Moreover, each hormone has distinctive physico-chemical properties that limit extraction efficiency in a given solvent. These obstacles have partially been overcome by the advancement of instrumentation technologies, which allowed the simultaneous analysis of a wide range of hormones with high sensitivity and selectivity. For hormone profiling analyses, several mass spectrometry-based methods have been developed with state-of-the-art instruments such as GC-MS or UPLC-ESI-MS/MS that enable the quantitative analysis of secondary metabolites (Chiwocha et al., 2003). By virtue of these analytical tools, more sophisticated studies can be designed to investigate regulatory networks existing between different endogenous hormones in plants. For my thesis I decided to work on three groups of hormones - IAA, ABA and CK - which are known to display cross-talk and interact in determining root system architecture (RSA) (Figure 1).

\section{Natural variation studies in Arabidopsis thaliana}

Genetic variation can be defined as the molecular polymorphism that is present within wild as well as in domesticated plants species (Alonso-Blanco et al., 2009). The analysis of natural variation in plant species may help to elucidate loci (genes) that are associated with important quantitative traits (Koornneef et al., 2004; Price, 2006). Due to the advances of molecular markers and statistical genomics, linkage mapping analysis has become an important tool to exploit genetic components responsible for polygenic traits. To facilitate these genetic analyses, many mapping populations have been developed in a variety of species (Brachi et al., 2010; Huang et al., 2011; Keurentjes et al., 2011; Kover et al., 2009). The principle of QTL mapping has also been applied to studies of transcript abundance (expression QTLs) and other intermediate molecular phenotypes (e.g., metabolic QTLs) (Carreno-Quintero et al., 2012; Cubillos et al., 2012; Feng et al., 2012; Keurentjes et al., 2008b; Kliebenstein, 2009; Lisec et al., 2009; Mackay et al., 2009; Takehisa et al., 2012; Toubiana et al., 2012; Wang et al., 2010). Metabolic profiling can be used for the identification of natural variants and genetic entities that contribute to shaping phenotypes and versatile biosynthetic pathways (Keurentjes et al., 2008a; Kliebenstein et al., 2001a; Kliebenstein et al., 2001b).

In the early 2000s, there was a period of rapid scientific progress in the extension of genomic resources. Rapid advances of whole genome sequencing (WGS) technology gave plant scientists access to full coverage of genetic information in model plants, such as Arabidopsis (2000) and important grain crop, such as rice (2002). Consequently, statistical approaches to find quantitative genetic elements in a species have been developed: association or linkage disequilibrium (LD) mapping using longstanding recombination outcomes (Clark et al., 2007; Kim et al., 2007; Nordborg et al., 2002; Nordborg and Innan, 2002). With the advent of high-throughput technologies for re- 
sequencing and high-density single nucleotide polymorphism (SNP) genotyping, genome-wide association (GWA) studies are rapidly becoming a leading method for dissecting the genetic architecture of complex traits in plants (Ingvarsson and Street, 2011; Weigel and Mott, 2009). Owing to high-density haplotype map information, present GWA studies have been applied to the model plant, Arabidopsis thaliana and some crop plants (Atwell et al., 2010; Buntjer et al., 2005; Ganal et al., 2009; Huang et al., 2012).

Advantages of using GWAS for mapping are that many alleles for each gene may be present, thus increasing the variation, and that the resolution of the mapping is higher. A disadvantage is the demand for a larger population size (it is estimated that at least 350 400 lines are required to obtain reliable data), which is a major drawback especially when expensive and/or time consuming analyses have to be done, and population structure may make the interpretation of data complicated (Atwell et al., 2010). Advantages and drawbacks of the GWA approach have been outlined in detail in various reviews (Alonso-Blanco et al., 2009; Koornneef et al., 2004; Weigel, 2012). As an alternative to GWAS, elaborate multi-parent based recombinant inbred line (RIL) populations were constructed (Huang et al., 2011; Keurentjes et al., 2011; Yu et al., 2008). They include an Arabidopsis multiparent RIL (AMPRIL) population and multi-parent advanced generation inter-cross (MAGIC). A draw

back of using multi-parent lines still lies in the required large population size needed to cover all recombination events $(\geq 500)$.

Bi-parental RILs are often chosen as an initial experimental material for the identification of QTLs. In Arabidopsis, advantages of linkage mapping using RILs are: 1. the availability of various populations, 2. the relatively small size of the population to be investigated (100 150 lines is sufficient for QTL mapping in Arabidopsis) and 3. such populations have already been studied for a wide range of phenotypic traits, thus allowing integration of prior data (Borevitz et al., 2002; Sergeeva et al., 2006). More than 60 Arabidopsis RIL populations are available from the world stock centres, and other types of inbred families, such as near isogenic lines (NILs) are also available (Weigel, 2012). Therefore, I chose the approach of using a RIL population, selected based on natural variation analysis of levels of endogenous hormones.

\section{Challenges in this thesis}

Despite extensive studies of quantitative phenotypic traits, several research topics have seldom been explored, for example, the variation of levels of plant hormones and application of that data to quantitative genetic analysis in plants. With regard to variation in the levels of hormones, two aspects should be prioritised when studied: determining the levels of hormones in different organs (roots and shoots) and possible interactions between hormones for regulating plant development. 


\section{A concern of quantitative analysis using UPLC-ESI-MS/MS: matrix interference}

Numerous reports describe simultaneous quantification of multiple plant hormones (Durgbanshi et al., 2005; Kojima et al., 2009; Pan et al., 2010). However, there is a limitation to the application of these methods directly in the present study, because I pursued the concomitant analysis of a range of different metabolic intermediates of hormones preferably with a simple purification step, which assures acceptable recoveries of the analytes. In this approach a serious drawback of the LC-MS detector is the so called matrix effect or matrix interference caused impurities in the sample. Matrix effects are observed as either ion suppression or ion enhancement compared to the mass/charge $(\mathrm{m} / \mathrm{z})$ signal of the analyte without any interference of impurity. The matrix effect occurs early in the ionization process in the LC-MS interface as the result of co-eluted matrix components, affecting the sensitivity and the detection capability. This is presented as a loss or gain in response, leading to unreliable quantitative results. Therefore, I set out to develop a series of proper methods, especially for extraction and purification. For this task, commercially available SPE sorbents retaining acidic and/or basic compounds with different ionic strengths were compared to establish a method that purifies targeted hormones from the crude extract (Chapter 2).

\section{Phenotyping of complex Arabidopsis roots}

The root system plays a pivotal role in the physical support for the shoot and nutrient uptakes from the soil. The root system is plastic and dynamic. Roots consist of various cells/tissues that build up different structures such as root apical meristem and root cap, where the biosynthesis of various hormones arises. Nothing is known about natural variation in plant hormone content in roots and how this affect root architecture. Therefore, I first focused on the root system to study the interaction between hormones and root phenotypic traits.

Root initiation and branching are well-known examples of plant developmental processes, controlled by plant hormones. In order to analyse correlations between hormone levels and root phenotypic traits in a relatively mature root stage, I introduced the term, "mature root unit (MRU), as a descriptive developmental unit in complex root structure. Subsequently, complex root architecture was described in newly conceptualized phenotypic traits from two dimensional root images. A set of ecotypes was selected, and target hormones were analysed in roots at one well-defined stage. Using these two sets of data, possible roles of plant hormones mediating root system architecture (RSA) are illustrated (Chapter 3).

\section{Linkage mapping analysis for QTLs of hormone levels}

The strength of my study is the use of genetic resources and natural variation to unravel the regulation of hormone levels and their metabolism. In order to understand genetic components underlying 
metabolism in a range of hormones, as a newly adopted tool I concentrated on the classical linkage mapping analysis. QTL analysis at the 50\% flowering stage allowed me to distinguish developmental specificity of loci in a single experiment. In addition, QTLs for metabolite-ratios in the biosynthetic pathways were analysed and provide new insights into the genetic regulations of plant hormone metabolism (Chapter 4 and Chapter 5).

\section{Outline of the thesis}

The thesis consists of six chapters including four experiment chapters. In Chapter 1 I introduce the importance of plant hormones for the regulation of plant responses to the environment and how my $\mathrm{PhD}$ study will contribute to furthering our understanding of this. Chapters 2 to 5 , as research articles, describe the major findings of the research.

In Chapter 2 I describe the methodological validation: how to quantitatively analyse various classes of plant hormones in leaves and roots of Arabidopsis using UPLC-ESI-MS/MS.

In Chapter 3 I analyse the natural variation in hormone levels in Arabidopsis roots and correlations with complex root architecture.

In Chapter 4 I use quantitative trait loci analysis of hormone levels in Arabidopsis roots and in Chapter 5 I use quantitative trait loci analysis of hormone levels in Arabidopsis leaves and pinpoint loci that are underlying several aspects of hormone metabolism in Arabidopsis.

Finally, in Chapter 6, the General discussion, all findings are discussed and integrated. 



\section{Chapter 2}

Methodological validation: quantitative analysis of various classes of plant hormones in leaves and roots of Arabidopsis using UPLC-ESI-MS/MS 


\begin{abstract}
The analysis of natural variation in a plant species sheds light on understanding the molecular bases of phenotypic differences related to adaptation and ecological significance. A study of natural variation in plant hormone levels demands high through-put and concise methods to handle the required large numbers of lines/accessions with sufficient precision. UPLC-ESI-MS/MS is a suitable instrumental tool to achieve this, but extraction and purification should be optimized prior to its application on a population scale. Here, we validated several key- points, critical for determining the levels of a wide range of plant hormones in Arabidopsis extracts based on single solid phase extraction (SPE) purification. A commercially available hydrophilic-lipophilic balance (HLB) sorbent was chosen to recover a chemically wide range of hormone analytes. Initially, substantial matrix interference was observed in the form of ion suppression and ion enhancement, which hindered the acquisition of reliable results. Dilution using a smaller starting sample (e.g., $2.5 \mathrm{mg}$ ) reduced the matrix effects considerably but additional measures were required for most of the analytes. Flushing the HLB-SPE column with acidic methanol was more effective to reduce matrix effect than acetonitrile. The present approach, based on a single SPE purification step, provides a methodological perspective for the analysis of a wide range of plant hormones in partially purified Arabidopsis samples with minor interferences of the matrix.
\end{abstract}

Key words: plant hormone analysis, matrix effect, LC-ESI-MS/MS, ion suppression, ion enhancement 


\section{Introduction}

Plant hormones are small organic molecules in plants, present at low concentration, that act as chemical messengers mediating a plethora of developmental processes. Plant hormones consist of different groups of diverse chemical structures that have a wide range of polarity, from strong acids (e.g., salicylic acid) to volatiles (e.g., ethylene). The amounts of endogenous hormones in plants are low and quantitative measurements are not easily achieved because of interference by impurities present in extracts. In addition, most hormone classes are metabolically complex due to the activity of anabolic and catabolic pathways (Hedden and Thomas, 2012; Weng, 2014). In many instances, plant hormones regulate developmental processes in complex interactions rather than acting alone. Thus, simultaneous analysis of various hormones is desirable to understand their integrative physiological roles in plant development (Jones et al., 2010; Unterholzner et al., 2015).

Crude plant extracts contain thousands of chemically diverse compounds, challenging researchers to develop reliable quantitative methods for the targeted analysis of hormonal compounds, usually through chromatographic separation (Verpoorte, 1998). In general, after the initial extraction process, two main steps are required to conduct hormone analysis for multiple targeted compounds: (i) purification using solid-phase extraction (SPE) columns and (ii) detection in the proper analytical instrument, such as a tandem mass spectrometry coupled with liquid chromatography (LC).

SPE is a reliable and fast method to purify extracts, to reduce interference of the targeted analysis of compounds of interest by other chemical constitutes. Appropriate SPE sorbents should be chosen by considering the chemical properties of the target compounds and the contaminants. To increase purification efficiency SPE purification on one column is often combined with another type of column with different chemical affinity. Fractionation using different polarity and ionic strength of aqueous eluates can be another way to retrieve the compounds of interest. Various purification schemes using these methodological choices have been developed to attain both satisfactory recovery rates and simultaneous quantification (Kojima et al., 2009; Svacinova et al., 2012). Since such stepwise approaches of fractionations based on multiple SPE columns generate large numbers of fractions to be analysed, it is costly and time-consuming. Such issues are increasingly prohibiting research progress when large numbers of plant samples have to be analysed, e.g., in genetic studies (Chen et al., 2014; Matsuda et al., 2015).

Electrospray ionization (ESI) mass spectrometry coupled with LC has been used to analyse a wide range of biological compounds in many research fields. Due to its ultra-high sensitivity to monitor ion fragments based on mass-to-charge ratios of organic compounds, tandem mass spectrometry has been applied to the simultaneous detection of multiple target compounds in 
phytochemical studies (Schafer et al., 2016; Tarkowska et al., 2014). However, during the vaporization stage in the mass analyser, co-eluting impurities can cause ion suppression or ion enhancement, so called matrix effects (MEs) (Taylor, 2005). These results in either decrease or increase, respectively, of the signal of the target compound compared to that of a chemical standard in pure solvent in the same concentration, resulting in unreliable quantitative data. ME has been evaluated for animal plasma and fluids (Van Eeckhaut et al., 2009), but it has been very little studied for plant extracts, although these are chemically complex, even after SPE purification, and thus have a high potential for displaying ME.

In the model plant Arabidopsis thaliana, the wealth of knowledge from natural variation studies has led to the understanding of molecular mechanisms for plant development, adaptation and speciation (Mitchell-Olds and Schmitt, 2006). Unravelling the genetic and biochemical bases of metabolic traits has been accelerated by recent natural variation studies coupled with advances of genotyping/sequencing technology (Alonso-Blanco et al., 2009; Chen et al., 2014), but little has been done on variation of hormone levels, even in Arabidopsis.

The study of natural variation for levels of endogenous plant hormones and their metabolites necessitates a high through-put purification strategy to deal with the large number of samples required in quantitative genetic studies. Variation in hormone levels may be relatively small compared to those in gene expression (Delker et al., 2010). Moreover, many of the compounds of interest are chemically unstable in aqueous solutions, requiring simplified and rapid quantitative analytical methods. Hence, we set out to develop and validate a simple purification scheme for simultaneous extraction and quantification of plant hormones, which can be used in quantitative genetics analyses. In this study we assessed several key-issues such as extraction efficacies, affinity properties of SPE sorbents to chemically diverse hormones, and evaluation of MEs occurring in partially purified samples, aiming to enable the study of natural variation in plant hormones in A. thaliana. Our results based on a single HLB-SPE purification platform provide a methodological perspective for the simultaneous analysis of a wide range of different hormones with satisfactorily reduced levels of matrix interferences in UPLC-ESI-MS/MS analysis.

\section{Materials and Methods}

\section{Plant materials and sampling}

For germination, Arabidopsis seeds (Columbia-0) were placed on wet filter paper in a Petri-dish at $4{ }^{\circ} \mathrm{C}$ for 4 days and subsequently sown in a $0.5 \mathrm{~mL}$ cylindrical plastic tube, from which the bottom had been cut off and that was filled with $0.5 \%$ agar in half strength Hoagland nutrient solution ( $\mathrm{pH} 5.5$ ). Seedlings were grown in hydroponics containers ( 70 plants per 10 litres, renewing the solution once a 
week). Plants were grown at $21^{\circ} \mathrm{C}$ during the light period $(10 \mathrm{~h})$ and at $18^{\circ} \mathrm{C}$ during the dark period (14 h). Light intensity and humidity were fixed at $125 \mu \mathrm{Mol} / \mathrm{m}^{2} \mathrm{~s}$ and $70 \%$ respectively. After 21 days of culture, rosette leaves and roots were harvested between the 5th and 8th hour during the 10 hours daytime for hormone analysis. The two largest rosette leaves of 4 plants were selected and pooled for a replicate. The roots were carefully dried with paper tissue and five to six root systems were combined for a replicate. Pooled samples were immediately frozen in liquid nitrogen, ground and freeze-dried for 24 hours.

\section{Hormone extraction}

A carefully weighed amount of lyophilized powder of leaves or roots was sonicated with $2 \mathrm{ml}$ of methanol : water : formic acid $(15: 4: 1, \mathrm{v} / \mathrm{v} / \mathrm{v})$, containing isotope-labelled internal standards (100 nM final concentration, OlChemIm Ltd, Czech) for $15 \mathrm{~min}$ and extracted by shaking for 2 hours at $4^{\circ} \mathrm{C}$ in darkness. After centrifugation at 2,000 rpm for $10 \mathrm{~min}$ (swinging bucket rotor-type, Model-The Centaur 2, MSE, UK), the supernatant was collected, and the pellet was re-extracted with $2 \mathrm{ml}$ of ethylacetate : formic acid $(19: 1, \mathrm{v} / \mathrm{v})$ for 2 hours at $4{ }^{\circ} \mathrm{C}$ in darkness. For the extraction efficacy test, isotope-labelled internal standards were dissolved in the first extraction solvents (either methanol or ethylacetate based). Pooled supernatants were evaporated to dryness under vacuum (SpeedVac Concentrator, Savant SC210A, Savant RVT5105, Thermo, US). The extract was suspended in $1 \mathrm{ml}$ of methanol : water : formic acid $(15: 4: 1, \mathrm{v} / \mathrm{v} / \mathrm{v})$ and purified on SPE columns.

\section{SPE purifications for recovery rate test}

All SPE columns were manufactured by Waters (30 mg cartridge ${ }^{-1}$, Oasis ${ }^{\circledR}$, US), except C18 (500 mg cartridge $^{-1}$, GRACE $^{\circledR}$, US. The different SPE sorbents were eluted as follows:

HLB and C18: $4 \mathrm{ml}$ of methanol was used for conditioning, followed by $4 \mathrm{ml}$ of water for equilibrating. After loading of the aqueous sample, the column was washed with $1 \%$ methanol in water. The compounds were eluted from the column with $4 \mathrm{ml}$ of $5 \%$ formic acid in methanol and evaporated to dryness under vacuum. For the purification of plant extracts using HLB (hydrophiliclipophilic balance), after loading of aqueous sample, the column was flushed with $1.5 \mathrm{ml}$ of water. The elution was done with $2 \mathrm{ml}$ of $1 \%$ formic acid in methanol, followed by $1.7 \mathrm{ml}$ of $0.05 \%$ formic acid in acetonitrile. In order to retrieve all the target hormone analytes, the two eluates were combined into the same tube and evaporated to dryness under vacuum.

$M C X: 2 \mathrm{ml}$ of methanol was used for conditioning, followed by $1 \mathrm{ml}$ of water for equilibrating. After loading of the aqueous sample, the column was washed with $1 \mathrm{ml}$ of $1 \mathrm{M}$ formic acid (pH 1.4) in water. The column was eluted with three eluents, $1 \mathrm{ml}$ of methanol, $1 \mathrm{ml}$ of $0.35 \mathrm{M} \mathrm{NH}_{4} \mathrm{OH}(\mathrm{pH}$ 
11.0 ) in water, and $1 \mathrm{ml}$ of $0.35 \mathrm{M} \mathrm{NH}_{4} \mathrm{OH}$ in $60 \%$ methanol. The three fractions were combined and evaporated to dryness under vacuum.

MAX: $1 \mathrm{ml}$ of $1 \mathrm{M}$ formic acid was used for conditioning, followed by $2 \mathrm{ml}$ of methanol for equilibrating. After loading the aqueous sample, the column was washed with $1 \mathrm{ml}$ of $10 \mathrm{mM}$, $\mathrm{KH}_{2} \mathrm{PO}_{4}(\mathrm{pH}$ 7.0) in water and followed by $1 \mathrm{ml}$ of water. The column was eluted with two eluents, $1 \mathrm{ml}$ of methanol and $1 \mathrm{ml}$ of $2 \%$ formic acid in methanol. The two fractions were combined and evaporated to dryness under vacuum.

WCX: $2 \mathrm{ml}$ of methanol was used for conditioning, followed by $1 \mathrm{ml}$ of water for equilibrating. After loading of the aqueous sample, the column was washed with $1 \mathrm{ml}$ of $5 \% \mathrm{NH}_{4} \mathrm{OH}$ in water. The column was eluted with two eluents, $1 \mathrm{ml}$ of methanol and $1 \mathrm{ml}$ of $2 \%$ formic acid in methanol. The two fractions were combined and evaporated to dryness under vacuum.

$W A X: 2 \mathrm{ml}$ of methanol was used for conditioning, followed by $1 \mathrm{ml}$ of water for equilibrating. After loading of the aqueous sample, the column was washed with $1 \mathrm{ml}$ of $2 \%$ formic acid in water. The column was eluted with two eluents, $1 \mathrm{ml}$ of methanol and $1 \mathrm{ml}$ of $5 \% \mathrm{NH}_{4} \mathrm{OH}$ in methanol. The two fractions were combined and evaporated to dryness under vacuum.

\section{Quantitative analysis of plant hormones}

Quantitative analysis of hormones was conducted using an Acquity UPLC ${ }^{\circledR}$ System (Waters, US) coupled with a triple quadrupole mass spectrometer (Xevo ${ }^{\mathrm{TM}} \mathrm{TQ}$, Waters). Dried samples were resuspended in $200 \mu \mathrm{l}$ of acetonitrile : water : formic acid (5:95:0.1, v/v/v) and filtered through $0.45 \mu \mathrm{m}$ PTFE membrane (Phenomenex, US). Each sample was injected twice, onto an Acquity UPLC BEH $\mathrm{C}_{18}$ column (100x2.1 mm, $1.7 \mu \mathrm{m}$; Waters), with two different mobile phase schemes to separate target compounds: one for IAA, ABA and gibberellins (GAs), the second for cytokinins (CKs). For IAA, $\mathrm{ABA}$, and GAs, $20 \mu \mathrm{l}$ was injected and eluted by a binary gradient, consisting of $0.1 \%$ formic acid in water (A) and $0.1 \%$ formic acid in acetonitrile (B), for $11 \mathrm{~min}$ at constant flow rate $\left(0.5 \mathrm{ml} \mathrm{min}^{-1}\right)$ at $40^{\circ} \mathrm{C}$ of analytical column temperature. The linear gradient was performed as follows: $0 \sim 1.0 \mathrm{~min}, 5 \%$ of eluent B; $1.0 \sim 6.67 \mathrm{~min}, 5$ to $50 \%$ of eluent B; $6.67 \sim 7.33 \mathrm{~min}, 50$ to $90 \%$ of eluent $\mathrm{B} ; 7.33 \sim 9.0 \mathrm{~min}$, $90 \%$ of eluent $\mathrm{B} ; 9.0 \sim 9.5 \mathrm{~min}, 90$ to $5 \%$ of eluent $\mathrm{B}$. At the end of the gradient, the column was equilibrated to initial conditions for $1.5 \mathrm{~min}$. For CKs, $20 \mu \mathrm{l}$ was injected and eluted using a gradient with the same $\mathrm{A}$ and $\mathrm{B}$ mobile phases as above and at constant flow rate $\left(0.6 \mathrm{ml} \mathrm{min}^{-1}\right)$ at $40^{\circ} \mathrm{C}$ of column temperature for $14 \mathrm{~min}$. The linear gradient elution was performed as follows: $0 \sim 1.5 \mathrm{~min}, 0.2 \%$ of eluent B; $1.5 \sim 8.5 \mathrm{~min}, 0.2$ to $20 \%$ of eluent $\mathrm{B}$; $8.5 \sim 9.5 \mathrm{~min}, 20$ to $70 \%$ of eluent $\mathrm{B}$; $9.5 \sim 10.2 \mathrm{~min}$, $70 \%$ of eluent B; $10.2 \sim 10.5 \mathrm{~min}, 70$ to $0.2 \%$ of eluent B. At the end of gradient, the column was equilibrated to initial conditions for $3.5 \mathrm{~min}$. The effluent was introduced into the electrospray ion 
source (ESI) of the mass spectrometer with operating parameters: capillary voltage, $3 \mathrm{kV}$; cone voltage, $22 \mathrm{~V}$; source and desolvation temperature, $150{ }^{\circ} \mathrm{C}$ and $650{ }^{\circ} \mathrm{C}$, respectively; cone and desolvation gas flow, 50 and $1000 \mathrm{~L}$ hour $^{-1}$; MS mode collision energy, $2 \mathrm{~V}$; MS/MS mode collision energy, $10 \mathrm{~V}$. Per analyte, two selective transitions were used for multiple reaction monitoring (MRM). The MRM channels for each compound were grouped into time window clusters to reduce loss-of-signal during monitoring. Data were processed by TargetLynx in MassLynx ${ }^{\mathrm{TM}}$ Software (Version 4.1, Waters). The quantification of each target analyte was determined by a linear calibration curve that covered the range of concentrations of compounds in the samples, and then corrected by the recovery rate of deuteriumor ${ }^{13} \mathrm{C}$ isotope-labelled internal standards.

\section{Evaluation of MEs}

The MEs were evaluated as follows: spike solutions containing the same concentrations of both isotope-labelled internal standard and non-labelled standard (100 nM final concentration) for each target hormone were added into purified eluates in volumetric ratio of 1 to 1 . Ten microliters of the spiked solution were injected onto the UPLC system with above-given instrumental conditions. Peak areas of both the analyte and its internal standard were obtained and the percentage of ME was calculated with the following formula: Percentage of matrix effect $=\left(\frac{S-A}{C}\right) * 100$

where $\mathrm{S}=$ peak area of analyte in the spiked sample; $\mathrm{A}$ = peak area of analyte in the sample without the spike solution; $\mathrm{C}=$ peak area of standards in pure solvent.

\section{Results and Discussion}

\section{Comparison of SPE columns}

To analyse recovery rates, mixtures of 4 different groups of hormone standards (Fig. 1) were purified with seven kinds of SPE columns (see Materials and Methods for details). The HLB cartridge had a similar recovery rate as the $\mathrm{C} 18$ sorbent for the tested compounds except for $\mathrm{tZ}$ and DZR, which displayed higher and lower recovery rates on HLB, respectively (Fig. 2). The wide range of affinities of HLB to diverse hormone compounds results from the chemistry of its sorbent, consisting of a pyrrolidine moiety that provides both positive and negative charges. Because of these ambivalent chemical properties, HLB has been widely used to purify plant extracts containing diverse chemicals (Fontanals et al., 2007; Jeong et al., 2017; Kanaujia et al., 2007). In addition, HLB is advantageous to recover $\mathrm{tZ}$, a compound not retained by the $\mathrm{C} 18$ sorbent. Increasing the size of the $\mathrm{C} 18$ cartridge improved recovery rates for some of the tested hormones, for example, IAA, but not proportional to the increased size. 
Figure 1. Chemical structures of plant hormones analysed in this study.

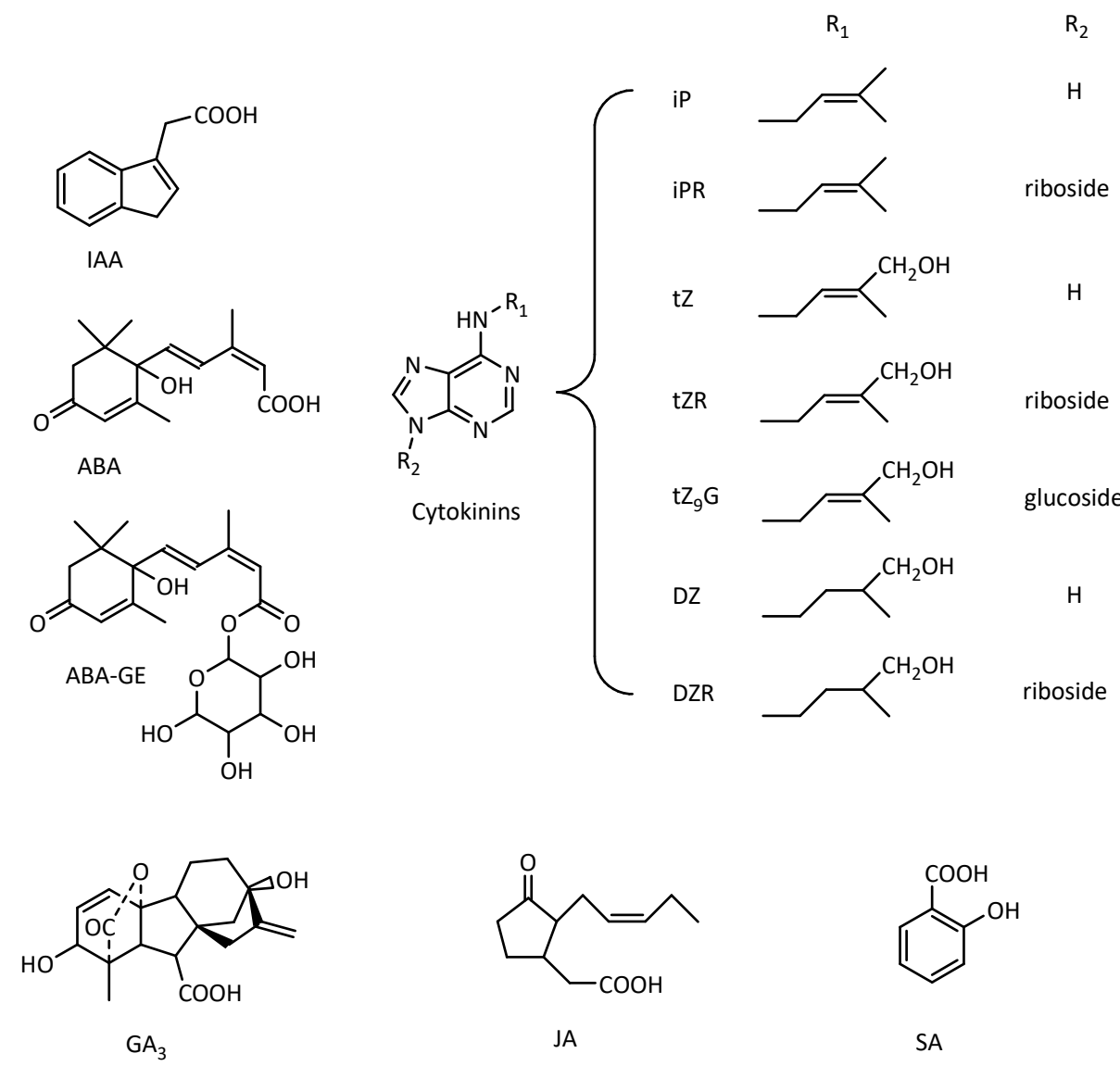


WCX, a mixed-mode weak cation exchange sorbent for strong bases and quaternary amines, displayed a recovery of about $50 \%$ for all of the six tested CK metabolites, but a very low recovery for acidic compounds, such as auxin and ABA. Both MAX and WAX that have anion exchange sorbent for acids presented substantial recovery rates (75 95\%) for IAA but had only weak affinity for $\mathrm{ABA}$ and $\mathrm{CK}$ metabolites. The poor recovery rate of MAX for ABA is in contrast to the study by Dobrev et al. (2005) who reported a high recovery rate (above $90 \%$ ) for ABA analysed with diode array UV-detector. Considering the differences of methodological conditions, it is assumed that the difference of recovery might be due to eluent conditions of used solvents and matrix interference, e.g., ion suppression in the mass analyser.
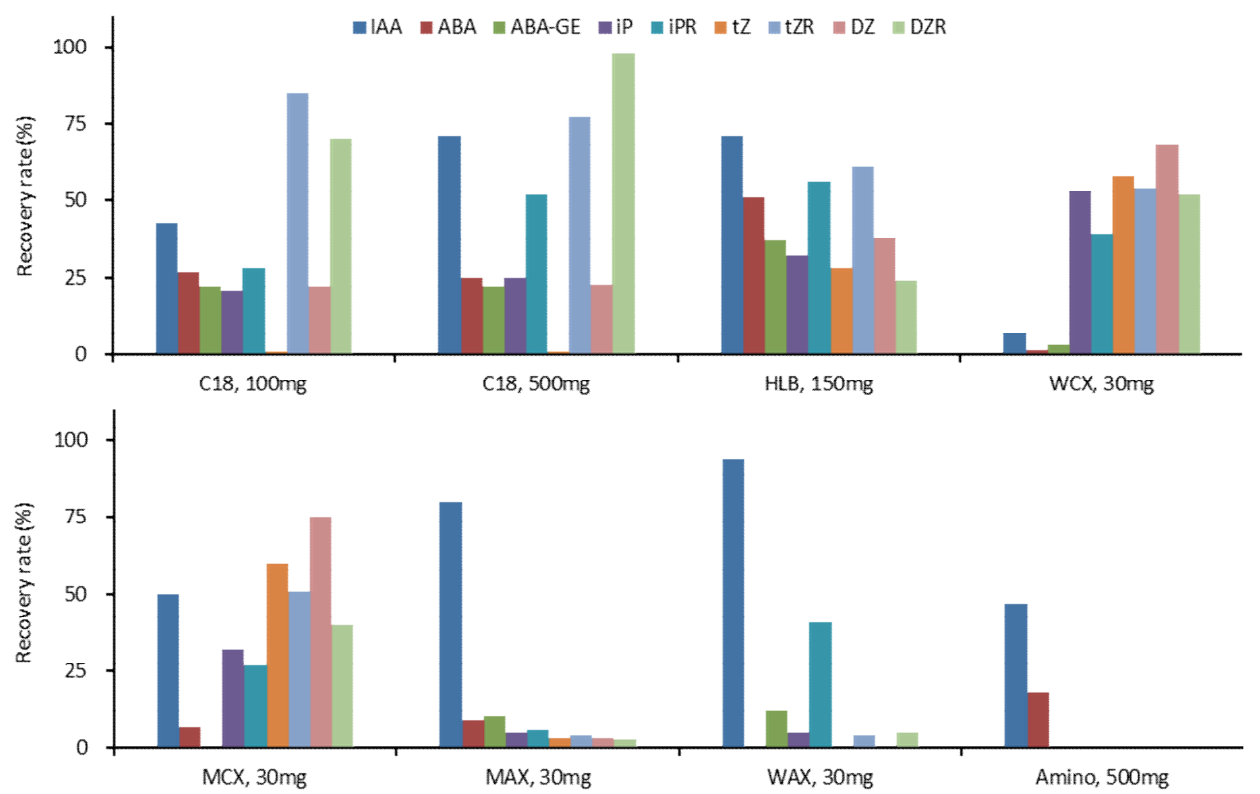

Figure 2. Recovery rates of hormones upon purification with various SPE sorbents. X-axes indicate SPE sorbents and their weights of packing material.

Since ABA and its catabolites are recovered well with hydrophilic SPE sorbents such as silica (Bai et al., 2018), a choice within polar sorbents is recommended for ABA although it belongs to the acid plant hormones. The amino SPE column only retained acidic compounds, such as IAA and ABA. Over all, these results show that HLB is the most versatile for the wide variety of target hormones and their metabolites, since a single purification strategy was desired. A one-step purification procedure will allow to analyse large series of plant samples, e.g. from accessions or segregating populations. 


\section{Efficiencies of extraction solvents}

For the extraction of a wide range of hormones from plant materials, usually acidified solvents are used. Moderate acids such as formic acid and acetic acid provide acidic forms of analytes, which are favourable for ionization in the mass spectrometer. For example, Bieleski buffer has been chosen as a standard extraction solvent in plant hormone analyses, especially for CKs (Chen et al., 2010; Hoyerova et al., 2006). In order to find an effective extraction solvent for a wide range of hormones, a number of different extraction procedures using Bieleski buffer and acidic ethylaceate as well as combinations were compared (Fig. 3). Two consecutive extractions with Bieleski buffer showed similar quantitative results with that of acidic ethylacetate in four different hormone groups, except for IAA. However, an extraction using Bieleski buffer followed by acidic ethylacetate resulted in higher recoveries of various hormones, such as ABAGE and some of the CKs. We accordingly concluded that two successive extractions using Bieleski buffer and acidic ethylacetate are recommended to increase yields of various hormones and their metabolites.

$\mathbf{E} \rightarrow \mathrm{B} \quad \mathrm{B} \rightarrow \mathrm{E} \quad \mathrm{E} \rightarrow \mathrm{E}$
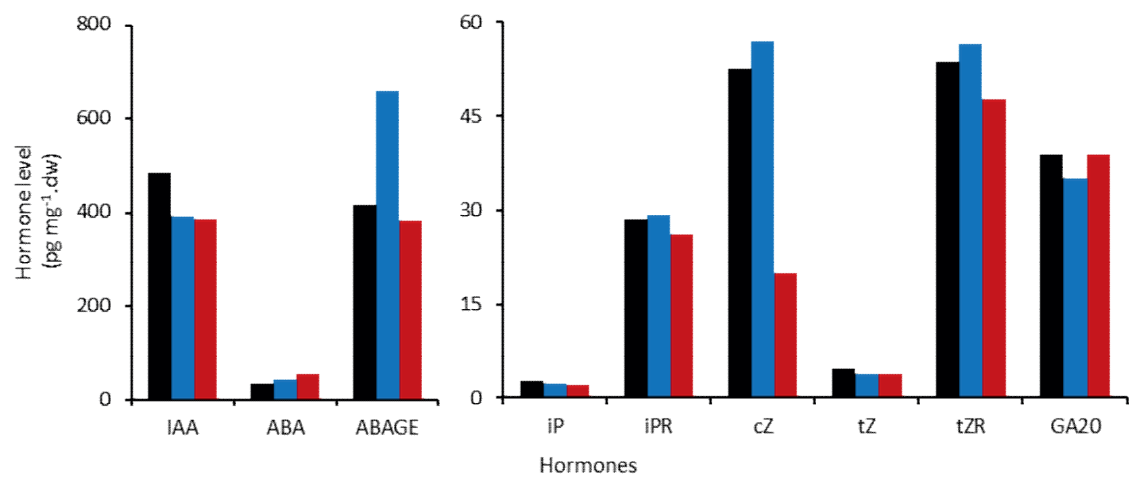

Figure 3. Comparison of extraction efficacies of different solvents. Lyophilized powder $(12.5 \mathrm{mg})$ of Arabidopsis roots (Columbia-0) was extracted and purified on an HLB column (packing size: 150mg). B indicates Bieleski buffer (based on methanol) and $\mathrm{E}$ indicates ethylacetate with $0.1 \%$ formic acid. See Materials and Method for details.

\section{ME interferences in LC-ESI-MS/MS analysis}

In LC-MS analysis, even after SPE purification, the presence of remaining impurities can cause signal distortion of analytes in the mass analyser, the so-called, matrix effect (ME), inducing responsive suppression or enhancement of ion fragments in the gas phase of droplets at the early stage of ionization, and resulting in unreliable quantitative results (Annesley, 2003; Jessome and Volmer, 
2006). Figure 4 shows two contrasting MEs, ion suppression and enhancement, for two isotopelabelled hormones, when they were added to a crude extract of Arabidopsis roots. In case of ABA, the signal value of the same amount of isotope-labelled internal standard in the crude extract was reduced by nearly $70 \%$ compared to that in the mobile phase solution (Fig. 4A). On the contrary, the internal standard of IAA represented ion enhancement, with an up to 1.8 times increased signal compared to that in the mobile phase solution (Fig. 4B). These two opposite results show that when plant extract samples are not properly purified, the signals of analytes in LC-ESI-MS/MS may not be representative of the real quantitative levels. When ion suppression and enhancement occur at target analytes, recovery rates of internal standards that elute closely to the target analytes may also be distorted.
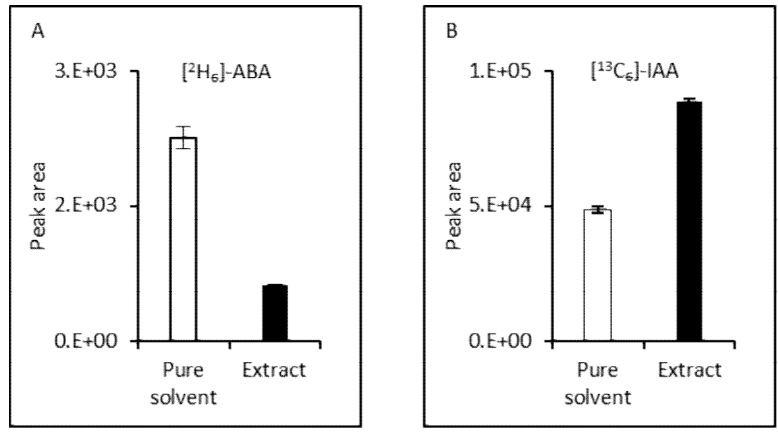

Figure 4. The influence of matrix effect on hormone quantification in Arabidopsis extract. (A) Ion suppression for the internal standard of ABA. (B) Ion enhancement for the internal standard of IAA. Isotopelabelled internal standards were added to pure solvent (methanol : water : formic acid $=5: 95: 0.1$, v/v/v) or a crude extract of Arabidopsis roots (Bayreuth-0, $15 \mathrm{mg}$ ). The root powder was extracted with Bieleski buffer and acidic ethyl acetate $(0.1 \%$ formic acid) consecutively. The dried extract was dissolved in a mixture of methanol : water $(1: 60, \mathrm{v} / \mathrm{v})$ with sonication for 30 minutes. Peak areas were obtained using UPLC-ESI-MS/MS analysis (see Materials and Methods for details).

Several strategies to reduce MEs in LC-MS analysis have been suggested. Chambers et al. (2007) validated that polymeric mixed-mode SPE purification using a combination of reversed and ion exchange sorbents strikingly reduced MEs in biological plasma samples. Dilution of samples can also be a useful method to reduce MEs. It was reported that the reduction of MEs for pesticide residues in plant extracts correlated to the logarithm of the concentration of co-eluting matrix components, suggesting that the higher the dilution, the better (Li et al., 2016; Stahnke et al., 2012). We analysed how the reduction of sample weight can alleviate MEs. To do this, we used HLB for purification and examined changes in quantitative levels using three different sample weights (Fig. 5). 
If there is any reduction in ME by reducing sample weight from 13 to 6.5 and $3.25 \mathrm{mg}$, quantifications of hormones will change by virtue of the reduction in impurities. For all four hormones tested (IAA, $\mathrm{ABA}, \mathrm{iP}$ and $\mathrm{tZ}$ ) higher quantities (expressed per $\mathrm{mg}$ tissue) were obtained with decreased sample weights. In cases of $\mathrm{ABA}$ and $\mathrm{tZ}$, the quantity increased 3 -fold when $3.25 \mathrm{mg}$ instead of $13 \mathrm{mg}$ was used.

It was surprising that the higher hormone quantities were obtained from lower sample weights, despite that the quantities were commonly presented as pico-gram per milligram of sample weight. In addition, except for IAA, recovery rates of $\mathrm{ABA}, \mathrm{iP}$ and $\mathrm{tZ}$ did not increase as sample weights were reduced (Fig. 5). To proof whether these results were caused by MEs, not by analytical inaccuracy, we evaluated MEs for individual compounds in the semi-purified extracts.
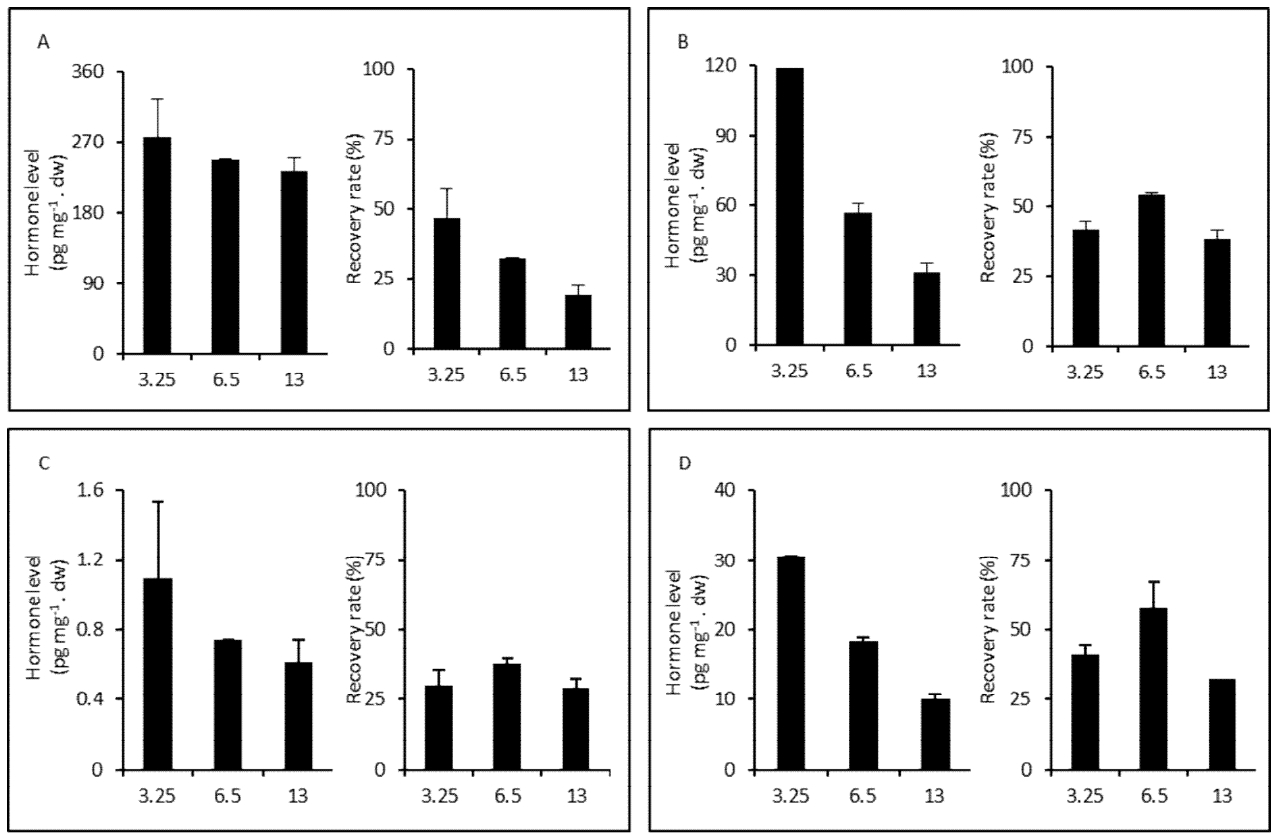

Figure 5. Effects of sample weight on hormone quantities and recovery rates in Arabidopsis root extracts.

(A) IAA. (B) ABA. (C) iP. (D) tZ. X-axes indicate sample weight. Hormone levels are presented as pg $\mathrm{mg}^{-1}$, dry weight of roots. Recovery rates were calculated using isotope-labelled internal standards for each hormone, for example $\left[{ }^{13} \mathrm{C}_{6}\right]$-IAA for IAA. 


\section{Evaluation of MEs based on post-extraction spiking}

Two leading methods have been suggested to evaluate MEs on quantitative analyses of phytochemicals using LC-MS: post-extraction addition and post-column infusion (Annesley, 2003; Taylor, 2005). We assessed the degree of ME present in leaf extracts using the post-extraction addition method (Van Eeckhaut et al., 2009). With this method, the relative abundances of ion fragments for exogenous analytes and their isotope-labelled internal standards added after SPE purification are compared to those in the mobile phase solution at the same concentration (Taylor, 2005). Figure 6 shows the degree of ME for the four different sample weights. As described above, the types of ME were different for the different analytes: ion suppression for $\mathrm{ABA}$ and $\mathrm{GA}_{3}$ and ion enhancements for IAA, $\mathrm{tZ}$ and $\mathrm{tZ9G}$.

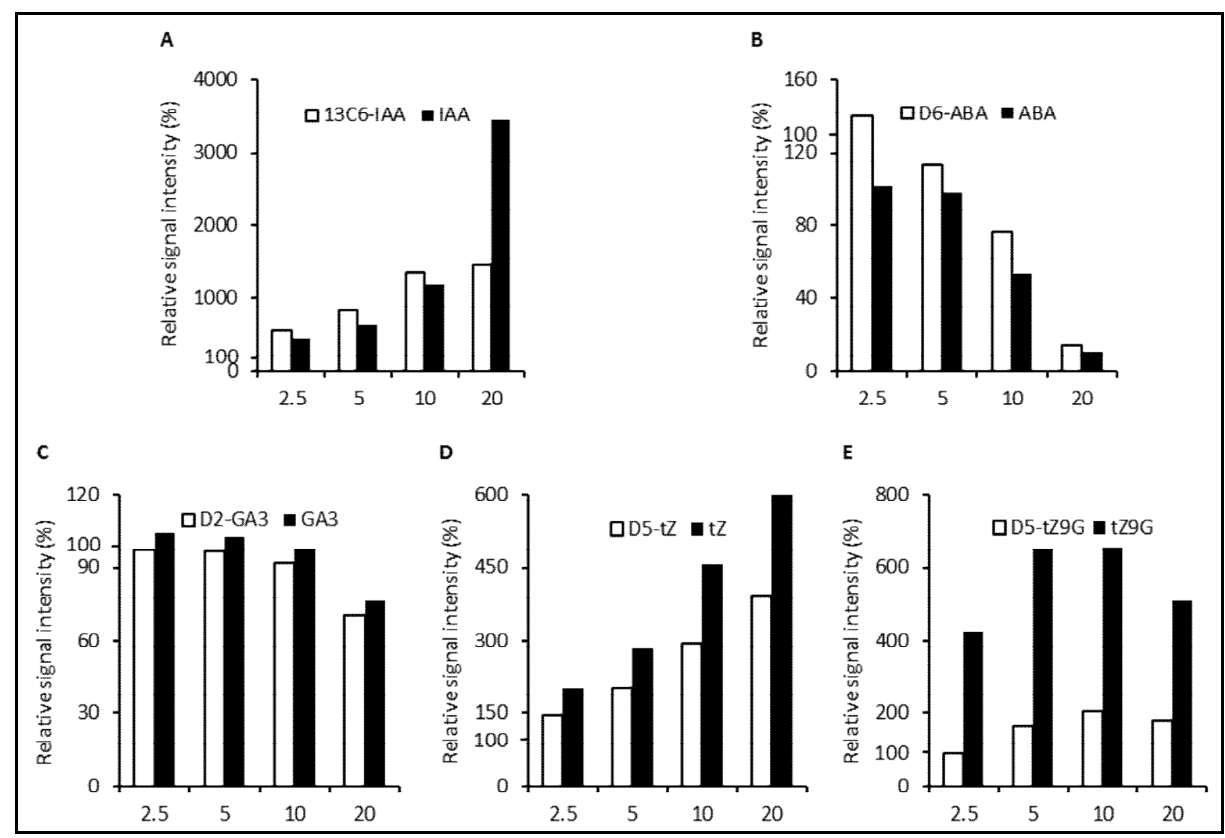

Figure 6. Effect of sample weight on matrix effect. (A) IAA. (B) ABA. (C) GA3. (D) tZ. (E) tZ9G. X-axes indicate sample weight (mg) used for extraction. Rosette leaves of Arabidopsis (Columbia-0) were used. The signal intensity of the analytes in pure solvent (methanol : water : formic acid $=5: 95: 0.1$, v/v/v) was set at $100 \%$.

In case of $20 \mathrm{mg}$ of leaf sample, signal intensities for IAA and $\mathrm{tZ}$ were 30 and 6 times higher, respectively, than those in pure solvent. These MEs were strikingly reduced as lower sample weights were used. For tZ9G, the degree of ion enhancement maximized when $10 \mathrm{mg}$ was used but rather decreased for $20 \mathrm{mg}$. This reduced signal at $20 \mathrm{mg}$ might be caused by the decrease of sensitivity in 
the mass analyser. For most analytes, the ME degrees differed between the analyte and its internal standard even though they eluted very closely together. These results show that degree of ME might be sensitive and changeable with co-eluted impurities within a deci-second RT frame: RTs for IAA and $\left[{ }^{13} \mathrm{C}_{6}\right]$ isotope-labelled internal standard were virtually the same at 2.54 minute but nevertheless there was a large difference in ME degree with the highest sample weight (Fig. 6). The effects of sample weigh on ion suppression were similar for $\mathrm{ABA}, \mathrm{GA}_{3}$ and their isotope-labelled standards, and close to zero when sample weights below 5 and $10 \mathrm{mg}$, respectively, were used. These results show that ion suppression can be substantially reduced through dilution of sample solution. The ion enhancement on IAA is more difficult to be solved effectively.
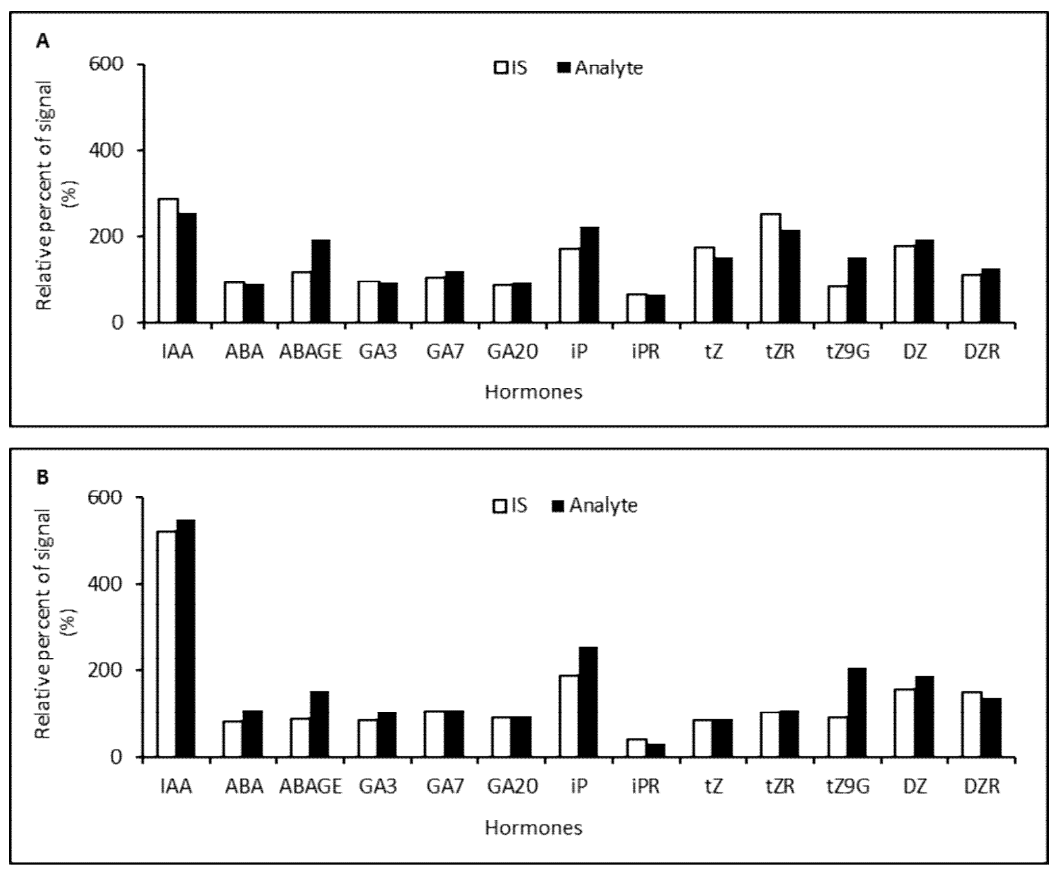

Figure 7. Degree of matrix effect observed in extracts of leaves and roots. (A) Degrees of matrix effect (ME) for hormone analytes and their internal standards observed in the extract of rosette leaves. (B) Degrees of ME for hormone analytes and their internal standards observed in the extract of roots. The signal intensity of the analytes in extracts is shown relative to the signal intensity of the analytes in the pure solvent (methanol : water : formic acid $=5: 95: 0.1, \mathrm{v} / \mathrm{v} / \mathrm{v}$ ), which was set at $100 \%$.

\section{Effects of different plant tissues on matrix interferences}

The chemical composition of plants varies according to plant species, organ and growing conditions (Shahidi et al., 1999; Tine et al., 2017; Wajs-Bonikowska et al., 2012). Since we are interested in hormone analysis in both roots and shoots we decided to analyse how ME differs between root and 
leaf extracts. Figure 7 shows that the over-all patterns and degrees of ME for each analyte in leaf extracts were similar to those in root extracts, except IAA, tZ and tZR. This suggests that the distinctively increased and decreased MEs result from differences in the concentration of co-eluting impurities. Hence, there is no guarantee that a purification/analytical LC-ESI-MS method optimised to reduce ME to zero for a particular extract will be equally effective for extracts prepared from different tissues/organs. With respect to determining quantification of analytes by using internal standards, serious errors may be introduced if the degree of ME for an analyte and its internal standard differ. In addition, since different genotypes of the same plant species may have different background of impurities in extracts, it is necessary to develop a proper purification method for the alleviation of ME.
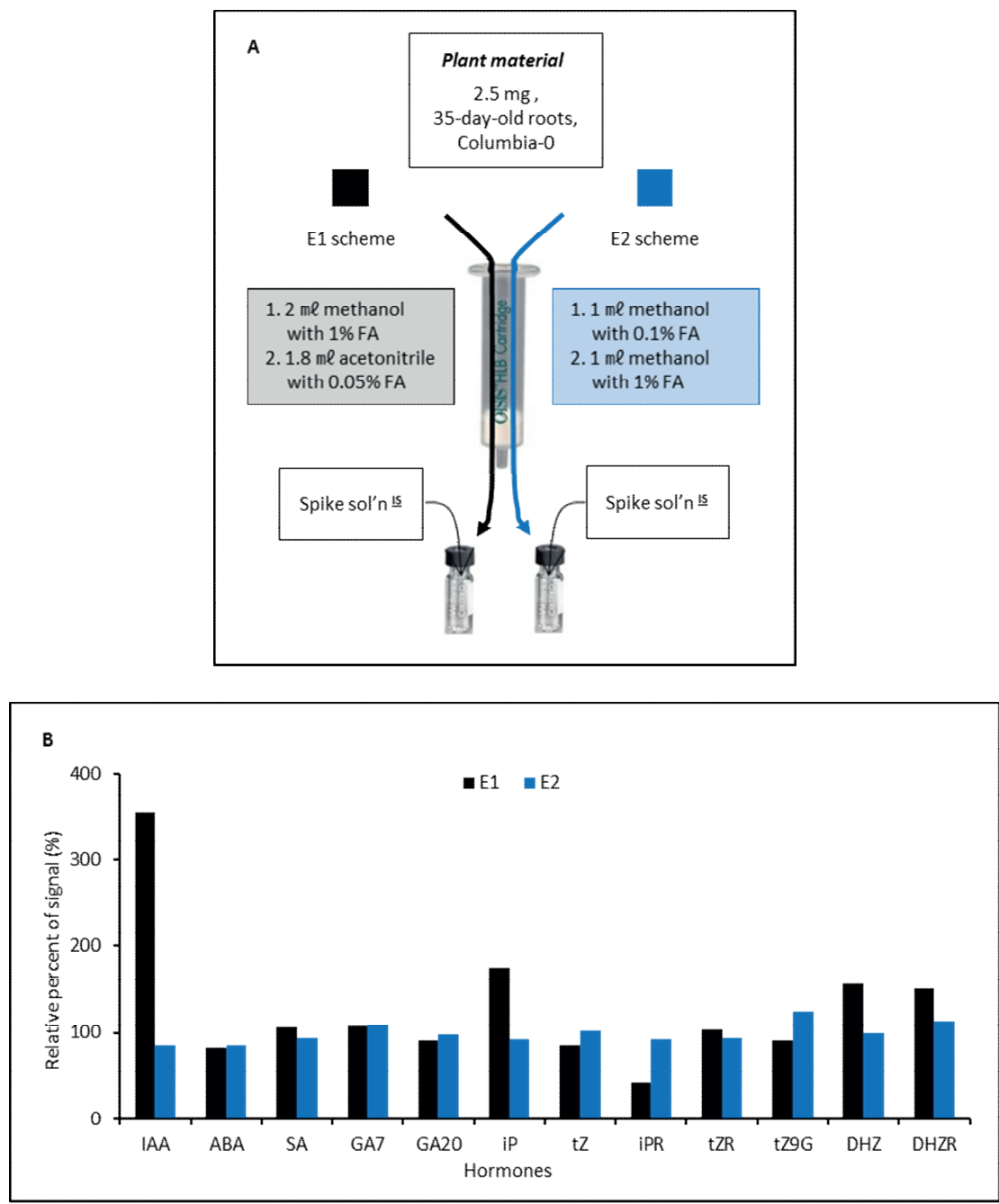
Figure 8. Effect of eluent solvent conditions on matrix effect. (A) Schematic representation of sample preparation. For E1, two sequential solvents based on methanol and acetonitrile were used. For E2, both solvents were methanol-based. For each elution scheme, the two sequential eluates were combined an then analysed on ESI-MS/MS. (B) Relative matrix effect (ME) observed in the two elution schemes (black bars for E1 and blue bars for E2). The signal intensity of the internal standard in pure solvent (methanol : water : formic acid $=5: 95: 0.1, \mathrm{v} / \mathrm{v} / \mathrm{v}$ ) was set at $100 \%$. The degree of ME was assessed based on the post-extraction spike method using internal standards.

\section{Effects of elution solvents on matrix interference}

Because impurities in the sample cause ME, the solvent used to elute the SPE cartridges will considerably affect the degree of ME. To assess this effect, we compared two elution schemes on the HLB column: E1 consisted of two sequential elutions with acidic methanol and acetonitrile (routinely used in the present study) and E2 which only used acidic methanol eluents (Fig. 8A). Despite that the polarity index of methanol (5.1) is similar to that of acetonitrile (5.8), this moderate decrease in the polarity of the eluent resulted in a considerably reduced matrix interference, with both ion enhancement and suppression being alleviated (Fig. 8B). This suggests that exclusion of polar compounds in the eluent is desirable to attain reliable response signals with low ME. Hence, it is recommended that when a SPE is used to purify plant extracts, eluting solvent condition should be carefully chosen by comparing degrees of ME according to different solvents on polarity

\section{Conclusion}

In this study, we have shown several methodological approaches related to extraction and purification aiming at reliable quantitative determination of diverse hormones in Arabidopsis extracts using UPLC-ESI-MS/MS. A key factor to make quantitative data of hormone levels reliable and accurate is the degree of ME (ion suppression and ion enhancement) that should ideally be close to zero. Here we propose that when a sample preparation protocol is being developed with regard to sample weight, extraction and SPE purification, also the degrees of ME for the target analytes should be evaluated and optimized. The following procedure can be recommended: i) select solvents for the elution of analytes from a SPE sorbent such that it results in negligible ion suppression/enhancement of target analytes, ii) adjust sample weight to ensure the lowest ME in combination with sufficient signal intensity of the target analytes, and iii) consider recovery rates only when the degree of ME is close to zero. We conclude that matrix effects in plant extracts, described as the "Achilles heel" of LC-ESItandem mass spectrometry (Taylor, 2005), should be critically assessed and suppressed, for which the present study give a number of suggestions. 


\section{[Supplementary Information]}

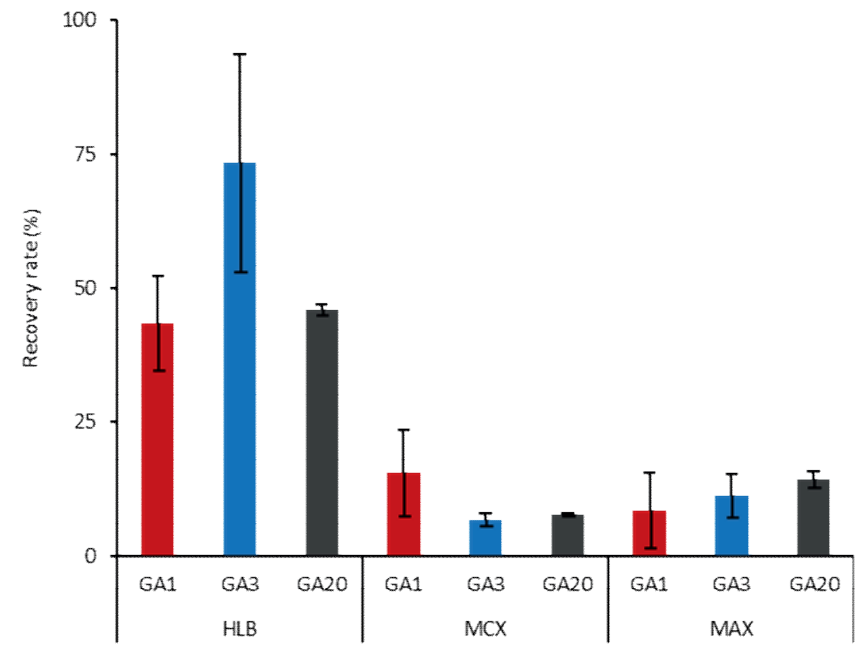

Figure S1. Recovery rates of isotope-labelled gibberellin standards on SPE sorbents. 



\section{Chapter 3}

\section{Natural variation of hormone levels in Arabidopsis roots and correlations with complex root architecture}

Sangseok Lee, Lidiya I. Sergeeva and Dick Vreugdenhil

Published in Journal of Integrative Plant Biology (2018), doi: 10.1111/ jipb.12617 


\begin{abstract}
Studies on natural variation are an important tool to unravel the genetic basis of quantitative traits in plants. Despite the significant roles of phytohormones in plant development, including root architecture, hardly any studies were done to investigate natural variation in endogenous hormone levels in plants. Therefore, in the present study a range of hormones were quantified in root extracts of thirteen Arabidopsis thaliana accessions using a UPLC triple quadrupole mass spectrometer. Root system architecture of the set of accessions was quantified, using a new parameter (mature root unit) for complex root systems, and correlated with the phytohormone data. Significant variations in phytohormone levels among the accessions were detected, but were remarkably small, viz., less than three-fold difference between extremes. For cytokinins, relatively larger variations were found for ribosides and glucosides, as compared to the free bases. For root phenotyping, length-related traitslateral root length and total root length - showed larger variations than lateral root number-related ones. For root architecture, antagonistic interactions between hormones, for example, IAA to transzeatin were detected in correlation analysis. These findings provide conclusive evidence for the presence of natural variation in phytohormone levels in Arabidopsis roots, suggesting that quantitative genetic analyses are feasible.
\end{abstract}

KEY WORDS: Phytohormones, natural variation, root system architecture, correlations, Arabidopsis thaliana 


\section{Introduction}

As sessile organisms, plants have to respond adequately to the environment they are growing in. Environmental cues are perceived by plants and translated into internal signals including phytohormones. These hormones, alone, or in interactions, regulate the growth and development of the plant, ensuring optimal survival and reproduction. Within a species, natural variation occurs for many traits, due to adaptation of populations to local environments (Alonso-Blanco et al., 2009). Natural accessions and sets of recombinant inbred lines (RIL), derived from such ecotypes, vary considerably for primary and secondary metabolite contents, such as for instance flavonoids in Arabidopsis seeds (Kliebenstein et al., 2001b; Routaboul et al., 2012). It thus seems logical to assume that natural variation will also be present for hormone contents in plants. Although some reports describe variation for the response to exogenous hormone application (Dobon et al., 2011; Kanno et al., 2010; Novakova et al., 2005; Pilet and Saugy, 1987) and for hormone levels in leaves and root exudates (Cardoso et al., 2014; Monchgesang et al., 2016; Nam et al., 2017), data on natural variation in endogenous hormone concentrations in Arabidopsis roots are absent.

Root initiation and branching are well-known examples of plant developmental processes, mediated by plant hormones. Lateral root initiation is controlled by hormone interactions (GarayArroyo et al., 2012; Tanimoto, 2005). Specifically, cytokinins (CK) and auxin are key phytohormones that regulate root development, vascular differentiation and gravitropism (Aloni et al., 2006). The observed auxin maximum in the root apical region, resulting from specific cellular localization of auxin efflux carriers (PIN proteins), suggests that an IAA gradient plays a significant role in the dynamics of root growth (Petersson et al., 2009; Tanimoto, 2005). CKs, acting as negative regulators of lateral root initiation, are known to perturb the auxin gradient by controlling PIN-dependent transport (Laplaze et al., 2007; Marhavy et al., 2011; Marhavy et al., 2014). Recently concentration gradients for different $\mathrm{CK}$ metabolites were found in the primary root zones and different cells/tissues (Antoniadi et al., 2015; Bielach et al., 2012). Despite a wealth of studies aimed at unravelling biological functions of phytohormones in various root developmental processes, at least the following points have not been answered yet, and will be the focus of the present study: 1. Is natural variation present for hormone contents, and if so, to what extent?; 2. How does this natural variation of phytohormone levels correlate with root system architecture (RSA)?

Recently many studies were published addressing effective methods for quantitative highthroughput root phenotyping, coupled with computational software (Clark et al., 2013; De Smet et al., 2012; Iyer-Pascuzzi et al., 2010; Slovak et al., 2014). In order to describe and quantify root traits in dicotyledonous species, for instance Arabidopsis thaliana, most descriptive traits are determined by 
two key components - the primary root and the lateral roots - concerning both lengths and numbers (Smith and De Smet, 2012; Tian et al., 2014).

Up to now, most studies on RSA in Arabidopsis have been conducted at seedling stages to avoid the complexity of measuring root traits in mature plants (Dubrovsky and Forde, 2012). The vertical agar plate, a common in vitro culture method for Arabidopsis, results in two-dimensional patterns of root growth and allows relatively easy scanning of roots. Such root phenotypings have been conducted exclusively to seedlings - younger than two weeks after germination - because of the space limitation in Petri dishes and difficulties to separate each individual root from others in case of a complex root system. Little attention has been given to more mature roots (older than 3 weeks), or root traits beyond the seedling stages.

Analysing the natural variation in phytohormone levels will be an important tool to increase our understanding of the genetic variation governing the molecular mechanisms of hormonal regulation in developmental processes (Korstanje and Paigen, 2002). Here we report the variation in endogenous phytohormone levels in roots of $13 \mathrm{~A}$. thaliana accessions. Simultaneously, the RSA was analysed using a new descriptive phenotypic trait coined Mature Root Unit (MRU), as a descriptive developmental unit. Our finding that natural variation in root phytohormones in Arabidopsis exists, suggests that quantitative genetic approaches are feasible to dissect the molecular elements responsible for phytohormone levels and their effect on changes in root architecture.

\section{Materials and Methods}

\section{Plant growth and sampling}

Thirteen Arabidopsis thaliana L. Heynh. accessions (An-1, Bayeuth-0, Bor-4, Bur-0, Columbia-0, Cvi-0, Est-1, Fei-0, Ler-0, RRS-7, Shahdara-0, Ts-1, Tsu-0) from the Arabidopsis Seed Stock of the Laboratory of Genetics at Wageningen University were used. For germination, seeds were placed on wet filter paper in a Petri dish at $4{ }^{\circ} \mathrm{C}$ in darkness for 4 days, seeds were then sowed on the top of 0.5 $\mathrm{ml}$ tubes, with the bottom cut off, and filled with $0.5 \%$ agar in half strength Hoagland's nutrient solution ( $\mathrm{pH}$ 5.5). Tubes with germinating seeds were placed in a hydroponics tank filled with half strength of Hoagland's nutrient solution ( $\mathrm{pH} 5.5,9$ L), renewing the solution once a week and approximately 70 plants per tank). Plants were kept at $21{ }^{\circ} \mathrm{C}$ during the light period $(10 \mathrm{~h})$ and at $18{ }^{\circ} \mathrm{C}$ during the dark period $(14 \mathrm{~h}$ ), from sowing (day 0) until harvesting. Light intensity and humidity were fixed at $125 \mu \mathrm{mol}$ photons $\mathrm{m}^{-2}$ per second and 70 percent, respectively.

To avoid that individual root systems get entangled with roots from neighbouring plants, each whole root system was comparted individually in a plastic column (diameter $3 \mathrm{~cm}$, depth $6 \mathrm{~cm}$, open at top and bottom) in the hydroponics tank (Figure S1). After 23 days of culture, roots were harvested 
between the 5th and 8th hour during the 10 hours light period for hormone analysis and phenotyping. Root length and weight were measured. Thereafter, roots of five to six plants were pooled for a biological replicate. Four biological replicates of each accession were used for hormone analyses. Pooled roots were ground in liquid nitrogen and freeze-dried for 24 hours. Ten intact roots of each accession, which were grown at the same batch as for hormone analyses, were also harvested, put to Petri dishes filled with water and preserved at $-20^{\circ} \mathrm{C}$ until two-dimensional phenotyping.

\section{Two-dimensional root image and phenotyping}

Frozen roots were de-frosted in two steps to prevent roots from being broken into pieces: at $4{ }^{\circ} \mathrm{C}$ for two hours and after that at room temperature $\left(\sim 22{ }^{\circ} \mathrm{C}\right)$. Roots were spread in square Petri-dishes $(12.5$ $\times 12.5 \mathrm{~cm}$ ), containing $0.1 \%$ Tween 20 in water. Each branch root was unravelled from other roots with a brush in such a way that the whole root system was kept intact without any overlapping among roots. Two-dimensional root images were generated by a photo scanner (Epson, Perfection V700). The software package, WinRhizo (Regent Instruments Inc., Canada), was used to measure total root length (TRL, cm) from the resulting TIF file image. Total root-tip number (TRTN) was counted based on all emerged roots that were longer than $0.5 \mathrm{~mm}$ in length. In most cases identifying the primary root was not possible, since lateral roots had formed, which were indistinguishable from the primary root in length and diameter. Therefore, we introduced a new descriptor, as a basic unit of phenotyping, "mature root unit (MRU)" to measure complex root architecture (Figure 2). Lateral root number (LRN) and lateral root length (LRL) were assessed separately for each MRU. Within each MRU, lateral roots were further divided into four sections of equal length from top to bottom, and numbers $(\mathrm{N})$ and lengths $(\mathrm{L})$ were determined, resulting in these parameters: LRN-1Q/2Q/3Q/4Q and LRL$1 \mathrm{Q} / 2 \mathrm{Q} / 3 \mathrm{Q} / 4 \mathrm{Q}$. Traits of the secondary lateral roots attached to the lateral roots were designated as $2^{\prime}-$ LRN and 2'-LRL. To measure real length of roots in scanned images, the ruler dimension of ImageJ was corrected based on dimensions of the Petri dish. After blotting the roots with soft paper tissues, root fresh weight (RFW, mg root $^{-1}$ ) was determined in three to four replicates, each replicate consisting of roots from five to six plants. For the experiment on the dynamics of hormone concentrations during development, also root dry weight (RDW, mg root $^{-1}$ ) was measured after 24 hours of freeze-drying.

\section{Sensitivity test of primary root growth towards exogenous IAA}

For germination, seeds (Ler-0, Col-0, Cvi-0) were placed on wet filter paper in a petri-dish at $4{ }^{\circ} \mathrm{C}$ in darkness for 4 days, germinated seeds were then sowed on vertical plates with half strength of MS media with $0.8 \%$ agar and $1 \%$ sucrose, containing IAA $(0.01 \mu \mathrm{M}$, or $0.05 \mu \mathrm{M})$. Plants were kept at $23{ }^{\circ} \mathrm{C}$ during the light period $(16 \mathrm{~h})$ and at $20{ }^{\circ} \mathrm{C}$ during the dark period $(8 \mathrm{~h})$, from sowing (day 0 ) 
until harvesting. Light intensity and humidity were fixed at $125 \mu \mathrm{mol}$ photons $\mathrm{m}^{-2}$ per second and 70 percent, respectively. Primary root lengths were measured after 10 days.

\section{Hormone extraction and purification}

For each accession, four biological replicates were used to extract and quantify endogenous hormones. Powder of lyophilized roots $(2.5 \mathrm{mg})$, as a replicate, was sonicated with $2 \mathrm{ml}$ of methanol : water : formic acid $(15: 4: 1, \mathrm{v} / \mathrm{v} / \mathrm{v})$, containing isotope-labelled internal standards (each chemical, $100 \mathrm{nM}$ final concentration, OlChemIm Ltd, Czech, see supplementary data, Table S1) for $15 \mathrm{~min}$ and extracted by shaking for 2 hours at $4{ }^{\circ} \mathrm{C}$ in darkness. After centrifugation at $1200 \mathrm{~g}$ for $10 \mathrm{~min}$ (swinging bucket rotor-type, Model-The Centaur2, MSE, UK), the supernatant was collected, and the pellet was re-extracted with $2 \mathrm{ml}$ of ethylacetate : formic acid $(19: 1, \mathrm{v} / \mathrm{v})$ for 2 hours at $4{ }^{\circ} \mathrm{C}$ in darkness. Pooled supernatants were evaporated to dryness under vacuum (SpeedVac Concentrator, Savant SC210A and refrigerated vapour trap, Savant RVT5105, Thermo, US). The extract was suspended in $1 \mathrm{ml}$ of methanol : water : formic acid (15:4:1, v/v/v) and was purified on an Oasis-HLB column (150 mg, Waters, US) using the following procedure: the column was activated with $6 \mathrm{ml}$ of methanol, followed by $4 \mathrm{ml}$ of water. After loading the sample, the column was washed with $1 \mathrm{ml}$ of water. Phytohormones were eluted with two sequential eluents; $1 \mathrm{ml}$ of methanol : formic acid (99.9:0.1, v/v) and $2 \mathrm{ml}$ of methanol : formic acid (99:1, v/v). Both fractions were combined and dried under vacuum.

\section{Quantitative analysis of phytohormones}

Quantitative analysis of hormones was conducted using an Acquity UPLC ${ }^{\circledR}$ System (Waters, US) coupled with a triple quadrupole mass spectrometer (Xevo ${ }^{\mathrm{TM}} \mathrm{TQ}$, Waters). Purified samples were suspended in $200 \mu \mathrm{l}$ of acetonitrile : water : formic acid (5:95:0.1, v/v/v), filtered through $0.45 \mu \mathrm{m}$ PTFE membrane (Phenomenex, US) and injected onto an Acquity UPLC BEH $\mathrm{C}_{18}$ column $(100 \times 2.1$ $\mathrm{mm}, 1.7 \mu \mathrm{m}$; Waters). Two independent injections were chromatographed with different mobile phase schemes to separate targeted compounds: one for IAA, ABA and gibberellins, the second for CKs. For IAA, ABA and gibberellic acids, $20 \mu \mathrm{l}$ of sample were injected and eluted by a binary gradient, consisting of $0.1 \%$ formic acid in water (A) and $0.1 \%$ formic acid in acetonitrile (B), for $11 \mathrm{~min}$ at constant flow rate $\left(0.5 \mathrm{ml} \mathrm{min}^{-1}\right)$ at $50{ }^{\circ} \mathrm{C}$ of analytical column temperature for $11 \mathrm{~min}$. The linear gradient elution was performed as follows: $0-1.0 \mathrm{~min}, 5 \%$ eluent $\mathrm{B}$; $1.0-6.67 \mathrm{~min}, 5$ to $50 \%$ eluent $\mathrm{B}$; 6.67-7.33 $\mathrm{min}, 50$ to $90 \%$ eluent B; 7.33-9.0 $\mathrm{min}, 90 \%$ eluent B; $9.0-9.5 \mathrm{~min}, 90$ to $5 \%$ eluent $\mathrm{B}$. At the end of gradient, the column was equilibrated to initial conditions for $1.5 \mathrm{~min}$. For CKs, $20 \mu 1$ of sample was eluted by another mobile phase gradient with the same A and B mobile phases at constant flow rate $\left(0.6 \mathrm{ml} \mathrm{min}{ }^{-1}\right)$ at $50{ }^{\circ} \mathrm{C}$ of analytical column temperature for $14 \mathrm{~min}$. The linear gradient elution was performed as follows: $0-1.5 \mathrm{~min}, 0.2 \%$ eluent $\mathrm{B}$; $1.5-8.5 \mathrm{~min}, 0.2$ to $20 \%$ eluent $\mathrm{B}$; $8.5-9.5 \mathrm{~min}, 20$ to 
$70 \%$ eluent $\mathrm{B} ; 9.5-10.2 \mathrm{~min}, 70 \%$ eluent $\mathrm{B} ; 10.2-10.5 \mathrm{~min}, 70$ to $0.2 \%$. At the end of gradient, the column was equilibrated to initial conditions for $3.5 \mathrm{~min}$. The effluent was introduced in electrospray ionization source (ESI) of mass spectrometer with operating parameters: capillary voltage, $3 \mathrm{kV}$; cone voltage, $22 \mathrm{~V}$; source and desolvation temperature, $150{ }^{\circ} \mathrm{C}$ and $650{ }^{\circ} \mathrm{C}$; cone and desolvation gas flow, 50 and $1000 \mathrm{~L}^{\text {hour }}{ }^{-1}$; MS mode collision energy, $2 \mathrm{~V}$; MS/MS mode collision energy, $10 \mathrm{~V}$. Two selective transitions were used to perform multiple reaction monitoring (MRM) detections (Table S1). MRM for each compound was grouped into a few of distinct functions in order to reduce loss-of-signal during monitoring times. Data were processed by TargetLynx in MassLynx ${ }^{\mathrm{TM}}$ Software (Version 4.1, Waters). The quantification of each targeted analyte was determined using a linear calibration curve that covered the range of concentrations of compounds in samples and corrected by the recovery rate of the deuterium-labelled internal standard $\left(\left[{ }^{13} \mathrm{C}_{6}\right]\right.$ for IAA).

\section{Statistical analysis}

Correlations were calculated using XLSTAT (http://www.xlstat.com). A linear model of Pearson's coefficient was used for principal component analysis (PCA). Hierarchical cluster analysis was performed in R-program using hclust package.

\section{Results}

\section{Accessions and hydroponic culture}

Thirteen accessions were selected based on genetic variation (Horton et al., 2012), and on earlier observed variations in growth patterns and primary metabolites (El-Lithy et al., 2010). The set of accessions largely overlapped with collections used for natural variation studies of various traits (McKhann et al., 2004; Sutka et al., 2011). The first objective was to develop a reliable method of cultivation, in order to uniformly grow roots, suitable for phenotyping, and yielding sufficient biomass for the hormone analyses. $0.5 \mathrm{~mL}$ Eppendorf tubes, of which the tip and the lid were removed, were filled with $0.5 \%$ agar in half strength Hoagland's solution and used as seed support. Roots of seedlings easily grew through the agar and subsequently reached the hydroponic solution underneath (Figure S1). In comparison with spray-type aeroponics, hydroponics turned out to be more suitable for uniform root development (data not shown). In order to extend the period of vegetative development and to avoid physiological changes due to initiation of flowering, plants were grown under short days. Under the applied culture conditions, none of the 13 accessions showed visible signs of flowering transition before 40 days after germination. In a more extended time-course experiment with Col-0 and Ler-0, no sign of flowering was observed until 62 days. Twenty three day-old plants were used as they have a root system with multiple-order branching, but were still suitable for two- 
dimensional root phenotyping. At that time point, the accessions displayed a wide diversity in root architecture (Figure 1).

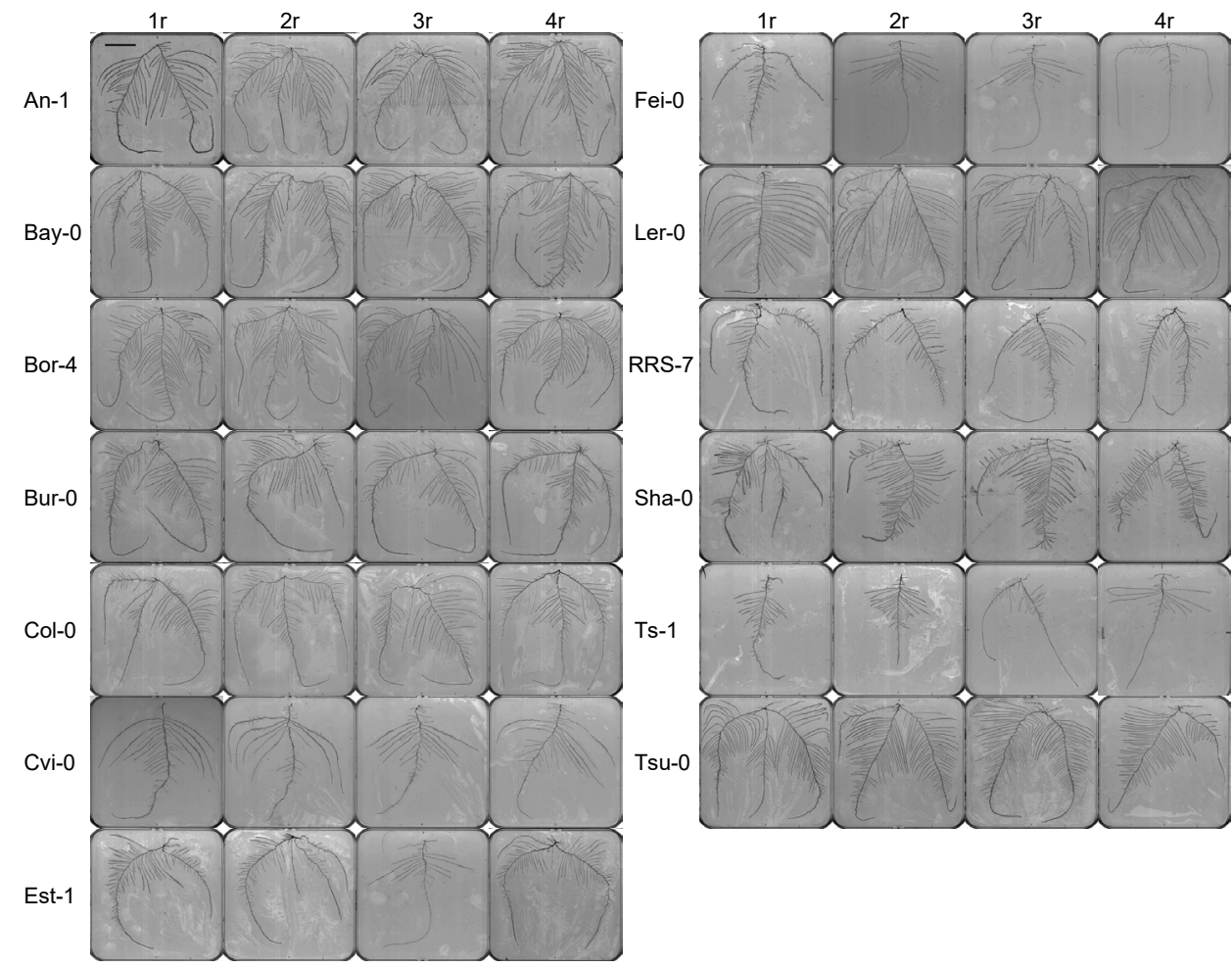

Figure 1. 2D-root images of 13 Arabidopsis accessions after 23 day of culture on hydroponics. From left to right, four replicates of each accession are presented. At the upper-left corner in the first replicate of An-1, the length of scale bar is $3 \mathrm{~cm}$.

\section{Parameters to quantify root traits}

Identifying the primary root with certainty was only possible for three accessions (Cvi-0, Fei-0, Ts-1), since the other accessions had several main roots with similar characteristics. Hence, we introduced the term "mature root unit (MRU)" as a basic unit for phenotyping RSA (Figure 2; the MRU number (MRUN) is 3, indicated with the ellipses). In this article, the term "main root" will be used for the longest root observed in each MRU, the term "lateral root" (LR) for the first-order branch roots attached to the main root in individual MRUs and the term "secondary lateral root" (2'-LR) for the second-order branch roots attached to "lateral root" in individual MRUs. Since in most accessions lateral roots were not evenly distributed along the main root, we quantified the distribution of lateral 
roots in individual MRUs, by dividing them into four sections of equal length, 1Q-4Q (Figure 2) and in each of these categories determined lateral root length (LRL) and number (LRN) (Figure 3).

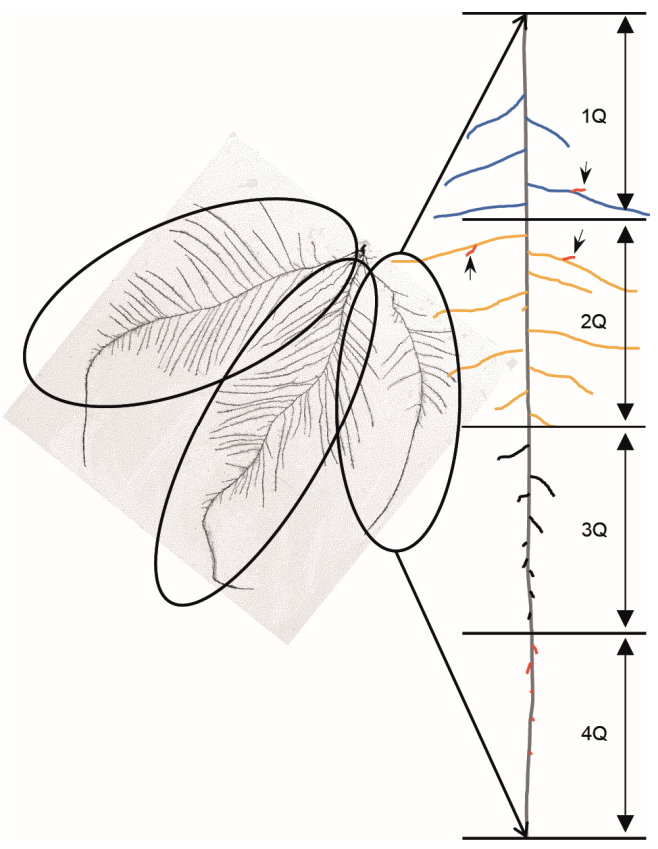

Figure 2. Schematic concept of mature root unit (MRU) and the quarterly distribution of lateral roots. In this example image, the root system consists of three mature root units (MRUs), indicated by the ellipses. A MRU on the right side is magnified in a linear way. Lateral roots alongside the main root axis are coloured differently (blue, yellow, black, red) in four quarterly panels from root base (1Q) to root tip (4Q). Secondary lateral roots ( $2^{\prime}-\mathrm{LR}$, orange colour, indicated by short arrows) are roots originating from lateral roots in MRUs.

\section{Variations of complex RSA}

In the quarterly division analysis of lateral root branching, LRL generally showed larger variation than LRN (Figure 3). This was observed in all four quarters and there was a tendency that the variation of these traits increased in the lower sections, where lateral roots have emerged later.

Other root phenotypic traits also varied between accessions, but not all to the same degree (Figure 4). Root fresh weight (RFW) showed more than five-fold difference between extremes (Cvi, Tsu), ranging from 0.5 to nearly $3 \mathrm{mg}$. Also mature root unit number (MRUN), total root length (TRL), total root-tip number (TRTN), LRL and secondary lateral root length (2'-LRL) displayed large variation. Smaller variation was observed for primary root length (PRL), LRN and lateral root density traits. Just as 2'-LRL showed larger variation than LRL, also 2'-LRN displayed a larger variation than LRN. Secondary lateral roots were present in most accessions, except in Ts-1, which also showed the 
shortest TRL. Among root traits, primary root length (PRL) showed the smallest variation. Lateral root density (LRD) showed limited variation since it was closely linked to lateral root number, which also showed small variation.
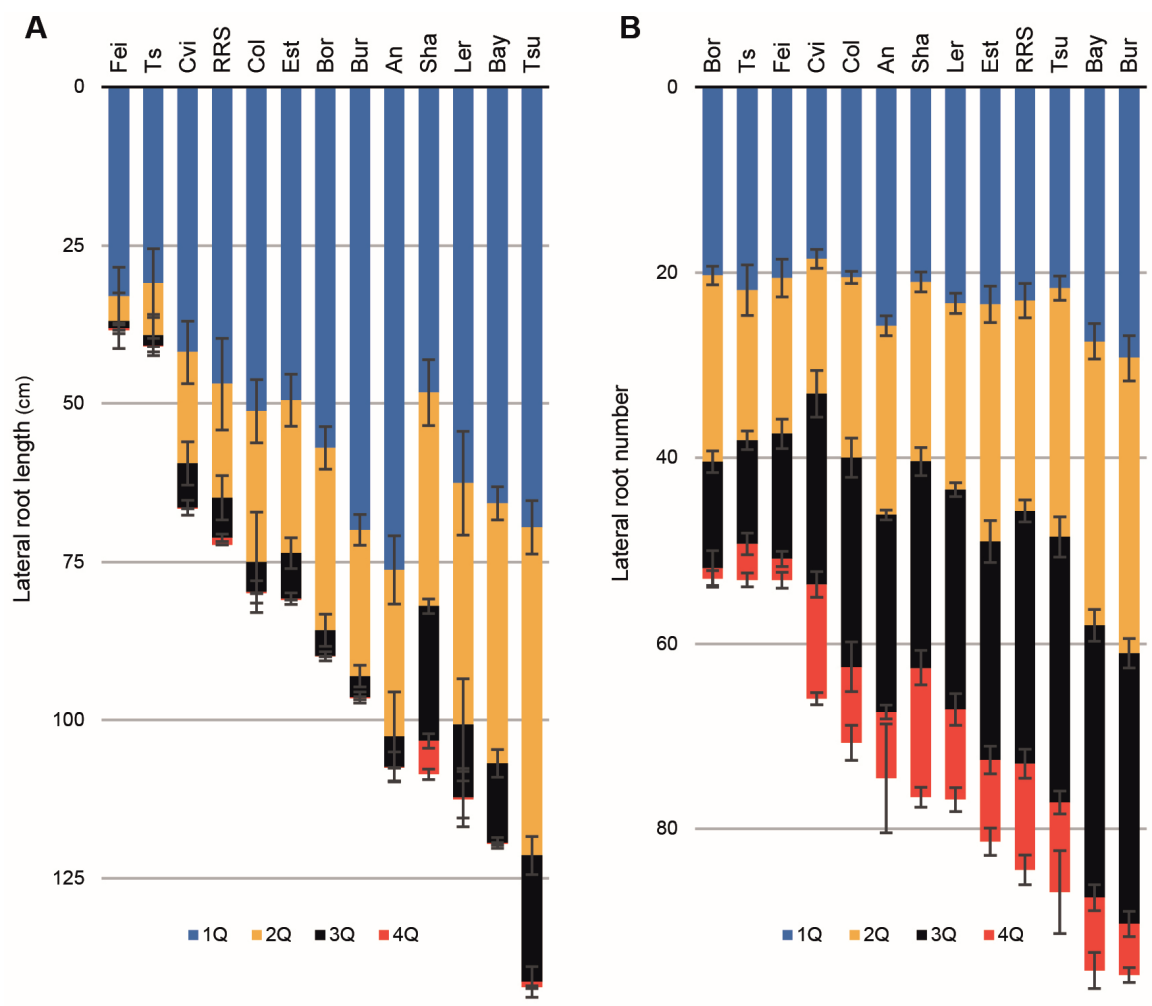

Figure 3. Quarterly distribution of lateral root length and lateral root number in 13 Arabidopsis accessions. (A) Lateral root length (LRL). (B) Lateral root number (LRN). Vertical bars on columns indicate standard errors $(\mathrm{n}=6 \sim 8)$, see legends in Fig. 2 for different colours.

RSA and their hierarchical relationships of 13 accessions in this study were obviously different from those of accessions analysed in previous studies, in which roots were grown on agar plates (Figure S2) (Armengaud et al., 2009; Kellermeier et al., 2013). Selection of accessions, method of culture and plant age in the present report differ too much from the cited studies to allow direct comparison.

Table 1 shows correlations between root phenotypic traits observed in 23-day-old roots of 13 natural accessions. Several obvious correlations were observed, e.g., between TRL and RFW. Our data allowed the study of more complex traits, including lateral-root related ones, and we will therefore focus on traits describing the complexity of the root system. RFW was highly correlated 
with the second quarter of lateral root length (LRL-2Q, $r^{2}=0.82$ ), followed by LRL-3Q $\left(r^{2}=0.7\right)$. TRL that is a determinant of RFW was the highest correlated with LRL-2Q, followed by LRL-1Q. These correlations imply that LRL-1Q and LRL-2Q contribute most to TRL. LRN was the highest correlated with LRL-3Q, followed by LRL-2Q, showing that Arabidopsis develops more lateral roots in the middle than in the top section of the main root. TRTN, an indicator of branch complexity in the whole root system, was the highest correlated $\left(r^{2}=0.97\right)$ with $2^{\prime}$-LRN, showing that phenotyping of mature root systems such as used in the present study requires sophisticated measurements including secondary and tertiary lateral root traits.
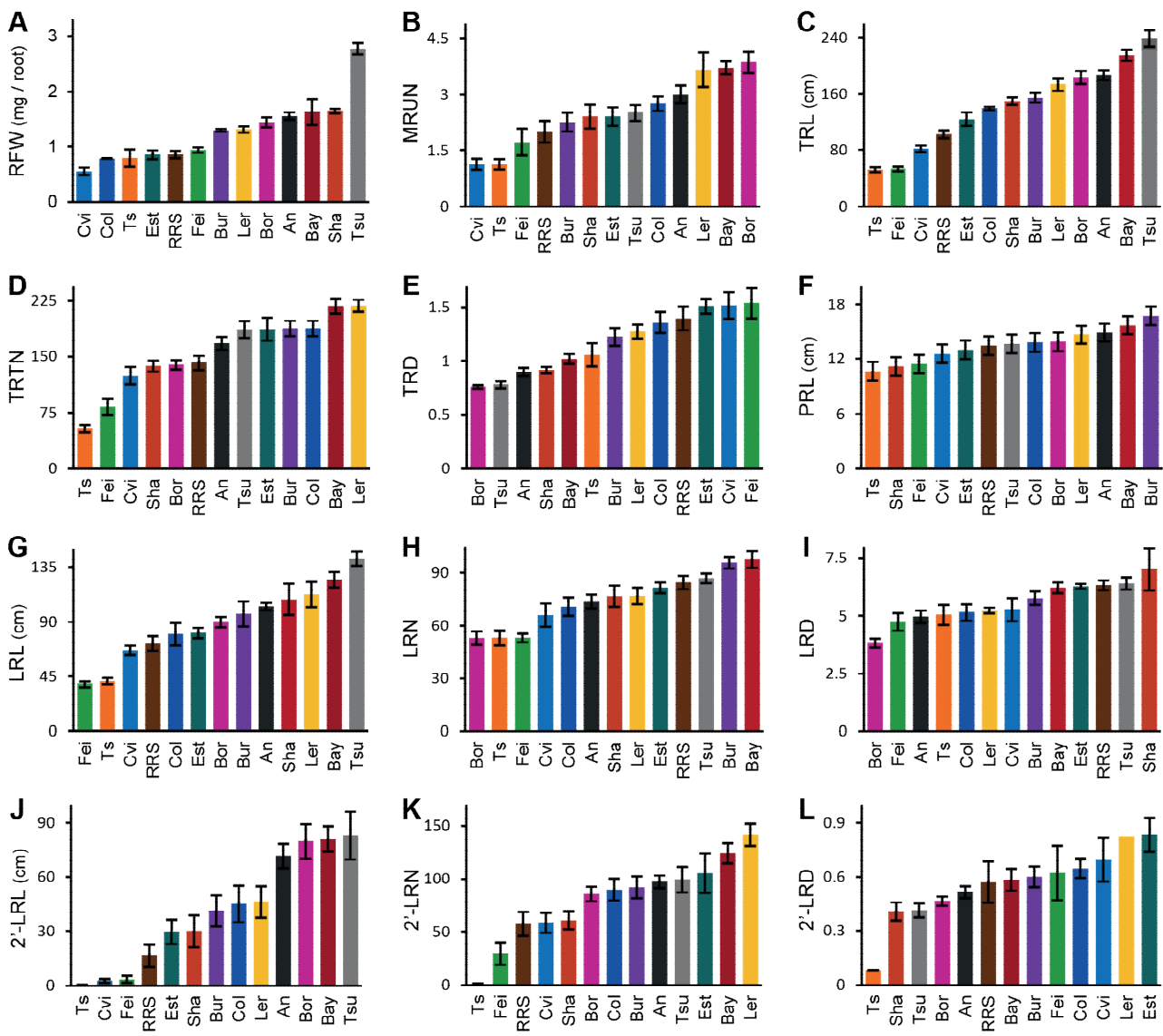

Figure 4. Natural variations of root system architecture (RSA) of 23-d-old roots. (A) Root fresh weight (RFW). (B) Mature root unit number (MRUN). (C) Total root length (TRL). (D) Total root-tip number (TRTN). (E) Total root density $\left(T R D=T R T N T^{-1}\right)$. (F) Primary root length $(P R L$, as the linear stretch of whole root system). (G) Lateral root length (LRL). (H) Lateral root number (LRN). (I) Lateral root density (LRD = LRN $\left.P R L^{-1}\right)$. (J) Secondary-lateral root length $\left(2^{\prime}\right.$-LRL). (K) Secondary-lateral root number ( $2^{\prime}$-LRN). (L) Secondarylateral root density $\left(2^{\prime}-\mathrm{LRD}=2^{\prime}\right.$-LRN TRL $\left.{ }^{-1}\right)$. 
Table 1. Correlations between root phenotypic traits. Significant correlations $(\mathrm{p}<0.01)$ are marked with an asterisk superscript. Abbreviations are: RFW, root fresh weight; MRUN, mature root unit number; TRL, total root length $(\mathrm{cm})$; TRTN, total root-tip number; TRD, total root density; PRL, primary root length as the linear stretch of whole root system; LRL, lateral root length; LRN, lateral root number; LRD, lateral root density; 2'LRL, secondary-lateral root length; 2'-LRN, secondary-lateral root number; 2'-LRD, secondary-lateral root density.

\begin{tabular}{|c|c|c|c|c|c|c|c|c|c|c|c|c|c|c|c|c|c|c|c|}
\hline variables & RFW & MRUN & TRL & TRTN & TRD & PRL & LRL & LRN & LRD & $2^{\prime}-\mathrm{LRL}$ & $2^{\prime}-$ LRN & $2^{\prime}-$ LRD & LRL-1Q & LRL-2Q & LRL-3Q & LRL-4Q & LRN-1Q & LRN-2Q & LRN-3Q \\
\hline$\overline{M R U N}$ & 0.45 & & & & & & & & & & & & & & & & & & \\
\hline TRL & $0.82^{*}$ & $0.79^{*}$ & & & & & & & & & & & & & & & & & \\
\hline TRTN & 0.45 & $0.71^{*}$ & $0.79^{*}$ & & & & & & & & & & & & & & & & \\
\hline TRD & $-0.72^{*}$ & -0.45 & $-0.68^{*}$ & -0.15 & & & & & & & & & & & & & & & \\
\hline PRL & 0.29 & 0.61 & 0.66 & $0.80^{*}$ & -0.17 & & & & & & & & & & & & & & \\
\hline LRL & $0.83^{*}$ & 0.66 & $0.96^{*}$ & $0.82^{*}$ & -0.59 & 0.58 & & & & & & & & & & & & & \\
\hline LRN & 0.40 & 0.29 & 0.58 & $0.79^{*}$ & -0.05 & 0.68 & $0.69^{*}$ & & & & & & & & & & & & \\
\hline LRD & 0.31 & -0.13 & 0.21 & 0.35 & 0.04 & -0.01 & 0.44 & $0.73^{*}$ & & & & & & & & & & & \\
\hline $2^{\prime}-\mathrm{LRL}$ & $0.75^{*}$ & $0.84^{*}$ & $0.95^{*}$ & 0.68 & $-0.73^{*}$ & 0.65 & $0.82^{*}$ & 0.40 & -0.02 & & & & & & & & & & \\
\hline $2^{\prime}-\mathrm{LRN}$ & 0.42 & $0.80^{*}$ & $0.80^{*}$ & $0.97^{*}$ & -0.18 & $0.77^{*}$ & $0.79^{*}$ & 0.63 & 0.16 & $0.72^{*}$ & & & & & & & & & \\
\hline $2^{\prime}-$ LRD & -0.20 & 0.32 & 0.16 & 0.64 & 0.55 & 0.45 & 0.20 & 0.36 & 0.08 & 0.09 & 0.67 & & & & & & & & \\
\hline LRL-1Q & 0.66 & 0.68 & $0.89^{*}$ & $0.82^{*}$ & -0.51 & $0.85^{*}$ & $0.84^{*}$ & 0.65 & 0.13 & $0.85^{*}$ & $0.80^{*}$ & 0.27 & & & & & & & \\
\hline LRL-2Q & $0.82^{*}$ & 0.66 & $0.93^{*}$ & $0.78^{*}$ & -0.59 & 0.47 & $0.98^{*}$ & 0.60 & 0.41 & $0.80^{*}$ & $0.76^{*}$ & 0.18 & $0.74^{*}$ & & & & & & \\
\hline LRL-3Q & $0.70^{*}$ & 0.26 & 0.59 & 0.44 & -0.41 & -0.02 & $0.76^{*}$ & 0.48 & $0.71^{*}$ & 0.38 & 0.38 & 0.02 & 0.33 & $0.80^{*}$ & & & & & \\
\hline LRL-4Q & 0.24 & -0.08 & 0.04 & -0.11 & -0.24 & -0.42 & 0.21 & 0.10 & 0.60 & -0.13 & -0.18 & -0.23 & -0.13 & 0.20 & 0.67 & & & & \\
\hline LRN-1Q & 0.23 & 0.33 & 0.40 & 0.56 & -0.12 & $0.75^{*}$ & 0.39 & $0.71^{*}$ & 0.26 & 0.37 & 0.45 & 0.11 & 0.64 & 0.23 & -0.05 & -0.20 & & & \\
\hline LRN-2Q & 0.48 & 0.41 & 0.62 & $0.71^{*}$ & -0.19 & $0.74^{*}$ & 0.62 & $0.86^{*}$ & 0.47 & 0.56 & 0.58 & 0.21 & 0.66 & 0.53 & 0.26 & -0.12 & $0.80^{*}$ & & \\
\hline LRN-3Q & 0.38 & 0.23 & 0.55 & $0.78^{*}$ & 0.03 & 0.64 & 0.68 & $0.97^{*}$ & $0.73^{*}$ & 0.35 & 0.62 & 0.44 & 0.62 & 0.60 & 0.50 & 0.09 & 0.57 & $0.76^{*}$ & \\
\hline LRN-4Q & 0.08 & -0.13 & 0.13 & 0.33 & 0.14 & -0.06 & 0.38 & 0.49 & $0.75^{*}$ & -0.12 & 0.23 & 0.31 & 0.09 & 0.38 & 0.67 & 0.56 & -0.11 & 0.01 & 0.60 \\
\hline
\end{tabular}

\section{Natural variation in hormone concentrations in roots}

In total, 33 phytohormones and related metabolites in four different classes were targeted for hormone quantification (Table S1). Eleven phytohormones—auxin, abscisic acid (ABA), gibberellin $\mathrm{A}_{9}\left(\mathrm{GA}_{9}\right)$ and eight CKs - could reliably be quantified in 23-day-old roots of all 13 accessions. Among 12 targeted gibberellins, four $\mathrm{GAs}\left(\mathrm{GA}_{1}, \mathrm{GA}_{3}, \mathrm{GA}_{7}, \mathrm{GA}_{20}\right)$ were detected in some accessions, while $\mathrm{GA}_{9}$ was commonly determined in all accessions.

Overall, phytohormone levels showed a limited range of variation among accessions (Figure 5). However, the extent of the variation strongly depended on the type of hormone and metabolites. Variations in the levels of auxin and $\mathrm{ABA}$ were within $\pm 25 \%$ from the average values for all accessions, and the difference between the extremes was less than twofold (Table S2). For CKs, the levels of isopentenyl adenine (iP) and trans-zeatin (tZ) were maintained in a very narrow range ( \pm $10 \%$ ), whereas CK ribosides showed relatively larger variations, viz., $\pm 50 \%$. Trans-zeatin glucosides (tZG) showed around $\pm 30 \%$ variation, larger than for the free bases. The variation in cis-zeatin (cZ) 
level was within $\pm 50 \%$, similar to the ribosides. GA9 displayed larger variation, up to threefold, between the extremes.
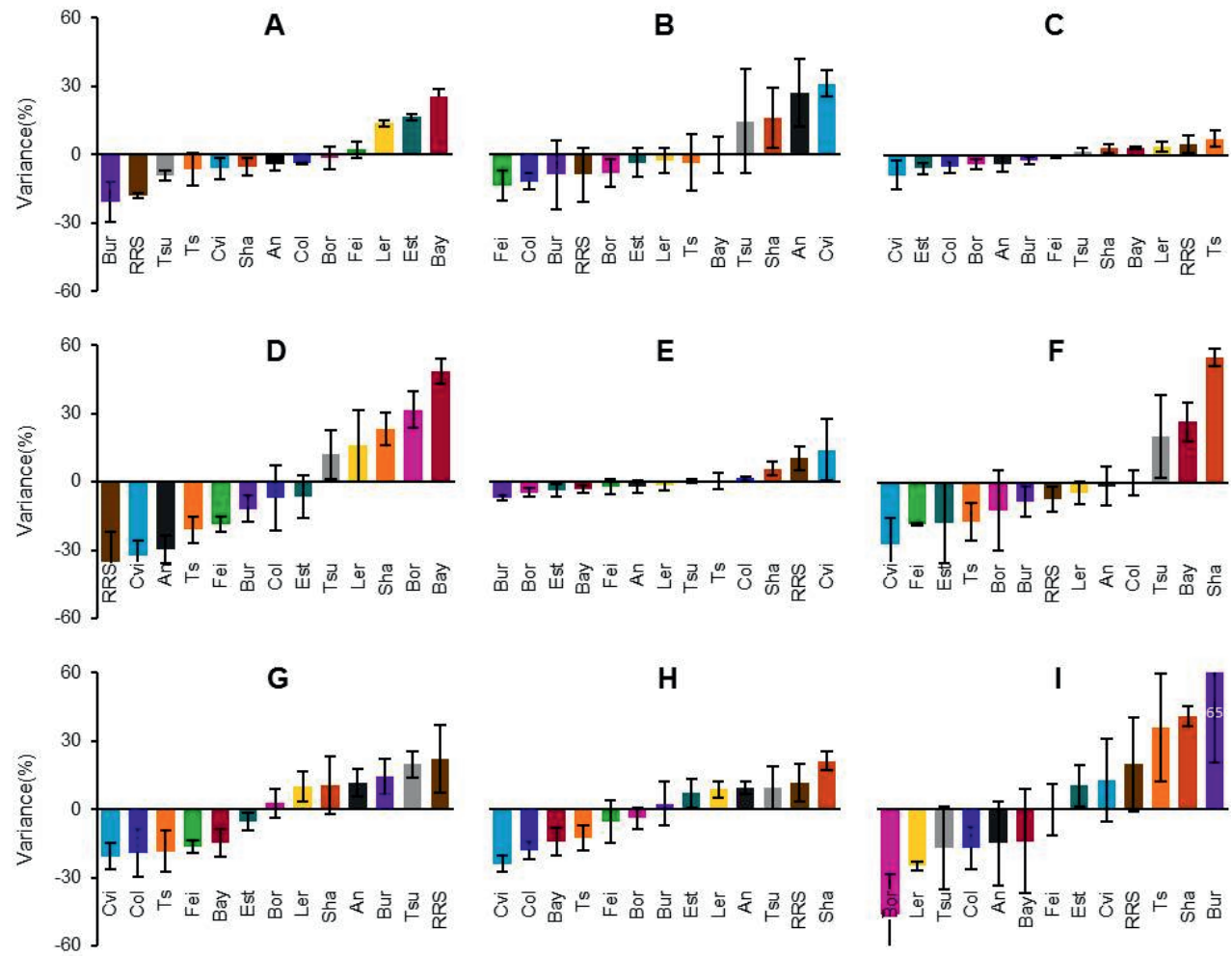

Figure 5. Natural variations of endogenous hormone levels in 23-d-old roots of Arabidopsis. (A) Indole-3acetic acid (IAA). (B) Abscisic acid (ABA). (C) Isopentenyl adenine (iP). (D) Isopentenyl adenine riboside (iPR). (E) Trans-zeatin (tZ). (F) Trans-zeatin riboside (tZR). (G) Trans-zeatin-7-glucoside (tZ7G). (H) Transzeatin-(O and 9)-glucosides $(\mathrm{tZ}(\mathrm{O}, 9) \mathrm{G})$. (I) Gibberellin A9 $\left(\mathrm{GA}_{9}\right)$. Y-axis is percentage of variance, obtained from the formula, $\mathrm{Y}=((\mathrm{X}-\mathrm{A}) / \mathrm{A}) 100: \mathrm{X}$ is the hormone concentration in a given accession and $\mathrm{A}$ is the average of concentration of hormone found in all accessions. Vertical bars on columns indicate standard errors $(n=3 \sim 4)$. tZOG and tZ9G were quantified together because peaks of compounds were overlapped in the chromatograms. See Table S2 for absolute quantities of hormones.

\section{Correlations between phytohormone levels and root system architecture}

Several significant correlations between phytohormone levels and RSA traits were found (Table 2). MRUN and TRTN, describing root maturity and global complexity, positively correlated with IAA level although the correlation was not significant. Secondary lateral root traits also positively correlated with auxin. However, RFW showed little correlation with auxin. These relationships reflect 
the important role of auxin in shaping the root system, particularly regarding lateral root branching and elongation.

Table 2. Correlations between hormone levels and RSA. Significant correlations are marked with an asterisk on bold figures: single and double asterisks indicate significance levels at $\mathrm{p}<0.05$ and $\mathrm{p}<0.01$, respectively. Blue and red colours indicate negative and positive correlations, respectively. Darker colours show higher correlations. Abbreviations of hormones are: IAA, indole-3-acetic acid; ABA, abscisic acid; iP, isopentenyl adenine; iPR, isopentenyl adenine riboside; cZ, cis-zeatin; tZ, trans-zeatin; tZR, trans-zeatin ribosides; tZ7G, trans-zeatin-7-glucoside; tZ(O,9)G, trans-zeatin-O and 9-glucosides; GA9, gibberellin A9. Refer to as the description of abbreviations for root phenotypic traits in Table 1.

\begin{tabular}{|c|c|c|c|c|c|c|c|c|c|c|c|c|c|c|c|c|c|c|c|c|}
\hline & RFW & MRUN & TRL & TRTN & TRD & PRL & LRL & LRN & LRD & $2^{\prime}-\mathrm{LRL}$ & 2-LRN & $2^{\prime}-$ LRD & $\begin{array}{c}\text { LRL- } \\
1 \mathrm{Q}\end{array}$ & $\begin{array}{c}\text { LRL- } \\
2 Q\end{array}$ & $\begin{array}{c}\text { LRL- } \\
3 Q\end{array}$ & $\begin{array}{c}\text { LRL- } \\
4 Q\end{array}$ & $\begin{array}{c}\text { LRN- } \\
1 \mathrm{Q}\end{array}$ & $\begin{array}{c}\text { LRN- } \\
2 Q\end{array}$ & $\begin{array}{c}\text { LRN- } \\
3 Q\end{array}$ & $\begin{array}{c}\text { LRN- } \\
4 Q\end{array}$ \\
\hline IAA & -0.01 & 0.49 & 0.21 & 0.38 & 0.10 & 0.07 & 0.18 & 0.07 & -0.01 & 0.24 & 0.47 & 0.37 & 0.04 & 0.26 & 0.14 & -0.17 & 0.09 & 0.10 & -0.01 & -0.05 \\
\hline$A B A$ & 0.07 & -0.30 & 0.04 & -0.05 & -0.03 & -0.12 & 0.14 & -0.02 & 0.11 & -0.06 & -0.05 & 0.03 & 0.08 & 0.12 & 0.28 & 0.15 & -0.27 & -0.32 & 0.06 & 0.51 \\
\hline iP & 0.26 & 0.01 & 0.01 & -0.11 & -0.25 & -0.19 & 0.10 & 0.13 & 0.33 & -0.06 & -0.19 & -0.54 & -0.12 & 0.13 & 0.29 & 0.32 & 0.20 & 0.10 & 0.06 & 0.04 \\
\hline iPR & 0.55 & $0.74^{* *}$ & $0.66^{*}$ & 0.48 & -0.54 & 0.24 & $0.63^{*}$ & 0.25 & 0.14 & $0.65^{*}$ & 0.52 & 0.02 & 0.35 & $0.69^{* *}$ & $0.56^{*}$ & 0.23 & 0.14 & 0.40 & 0.15 & -0.05 \\
\hline$c z$ & $0.69^{* *}$ & 0.06 & 0.45 & 0.22 & -0.48 & -0.12 & $0.63^{*}$ & 0.30 & $0.57^{*}$ & 0.23 & 0.16 & -0.23 & 0.28 & $0.66^{*}$ & $0.86^{* *}$ & $0.64^{*}$ & -0.10 & 0.08 & 0.35 & $0.59^{*}$ \\
\hline tz & -0.37 & -0.53 & -0.37 & -0.26 & 0.38 & -0.31 & -0.24 & -0.16 & 0.06 & -0.46 & -0.28 & 0.14 & -0.36 & -0.20 & 0.05 & 0.08 & -0.52 & -0.49 & -0.02 & 0.53 \\
\hline $\mathrm{tZR}$ & $0.64^{*}$ & 0.36 & $0.56^{*}$ & 0.33 & $-0.57^{*}$ & 0.04 & $0.67^{*}$ & 0.45 & $0.64^{*}$ & 0.43 & 0.25 & -0.19 & 0.34 & $0.65^{*}$ & $0.84^{* *}$ & $0.76^{* *}$ & 0.16 & 0.32 & 0.42 & 0.46 \\
\hline $\mathrm{tZ7G}$ & $0.57^{*}$ & 0.29 & 0.50 & 0.41 & -0.34 & 0.36 & 0.56 & 0.47 & 0.36 & 0.38 & 0.33 & 0.04 & $0.58^{*}$ & 0.47 & 0.41 & 0.31 & 0.34 & 0.42 & 0.47 & 0.27 \\
\hline $\mathrm{tz}(0,9) \mathrm{G}$ & 0.48 & 0.23 & 0.33 & 0.28 & -0.23 & 0.04 & 0.43 & 0.33 & 0.48 & 0.20 & 0.23 & 0.03 & 0.35 & 0.36 & 0.49 & $0.56^{*}$ & 0.24 & 0.26 & 0.30 & 0.30 \\
\hline $\mathrm{GA}_{9}$ & -0.28 & $-0.65^{*}$ & -0.45 & -0.27 & 0.30 & -0.15 & -0.28 & 0.27 & 0.51 & $-0.59^{*}$ & -0.46 & -0.24 & -0.27 & -0.38 & -0.04 & 0.37 & 0.29 & 0.15 & 0.22 & 0.27 \\
\hline
\end{tabular}

Most of the root traits, except TRD and LRN-4Q, negatively correlated with tZ, for which an antagonistic interaction with IAA in root development has been described (Pernisova et al., 2009; Ruzicka et al., 2009). Another active free base, iP showed different correlations with various root traits, viz., RFW, TRD, 2'-LRD and LRN-1Q/2Q, indicating that each free base may have different physiological effects on RSA. Cis-zeatin showed significant positive correlations with RFW, LRD, LRL-2Q/3Q/4Q and LRN-4Q, and its overall correlation pattern was similar with those of the ribosides and glucosides.

ABA negatively correlated with most of the root traits, except LRN-4Q, where very short lateral roots (shorter than $0.5 \mathrm{~mm}$ ) were dominant. Overall correlations of $\mathrm{GA}_{9}$ to each root trait were similar with those of $t Z$, except $L R N$ in the upper main root axis.

In order to have a global view of interactions between phytohormone levels and RSA, we performed a principal component analysis (PCA) based on Pearson's coefficients (Figure 6). Approximately, $58 \%$ of the variation was explained by the first two principal components (PC1, PC2), 
reaching $71 \%$ cumulative explanation with PC3 (Table S3). The first principal component (PC1) is composed mainly of all root traits and some CKs, such as tZR, iPR and tZ 7 G. The other phytohormones mainly contributed to PC2. CK ribosides and glucosides clustered closely with some of the root phenotypic traits, for example, RFW to $\mathrm{tZ}_{7} \mathrm{G}$. Trans-zeatin clustered with ABA and $\mathrm{GA}_{9}$, which were diagonally opposite to IAA.

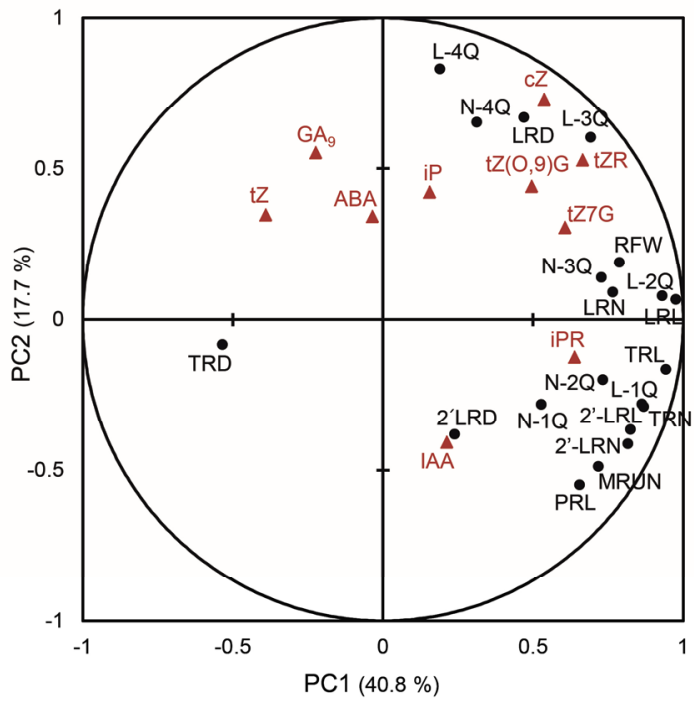

Figure 6. Principal component analysis of hormone levels and root system architecture (RSA) traits in 23d-old roots of Arabidopsis. Red-triangles indicate hormone traits and black circles show root phenotypic trait. Meanings of abbreviations are given in the legends of Figure 4 (root traits) and Figure 5 (hormones). For simplicity, LRL and LRN for quarterly distribution are abbreviated as L-1Q/2Q/3Q/4Q and N-1Q/2Q/3Q/4Q, respectively.

\section{Hormone levels versus hormone sensitivity}

The response of a plant developmental process to hormones is the result of hormone level and the sensitivity towards that hormone. In a pilot experiment we assayed the sensitivity for auxin of three accessions, which have been widely used for the generation of recombinant inbred lines (RILs). Since long term application under non-sterile hydroponic conditions will result in rapid breakdown of IAA, we used a sterile plate-assay instead. Figure 7 shows that roots of Ler and Col, in the absence of exogenous IAA, are longer than Cvi roots. This is consistent with various root-length related traits as measured for the same accessions in later developmental stages from the hydroponic system (Figure 4). The addition of IAA to the plates resulted in shorter roots. This would imply that IAA inhibits root 
elongation, a well-known phenomenon (Rahman et al., 2007; Swarup et al., 2007). Thus, if root elongation would only be controlled by the level of endogenous IAA, levels in Ler and Col would be expected to be lower than in Cvi. This is obviously not consistent with the data presented in Figure 5. Then, can differences in root length between these three accessions be explained by differences in sensitivities towards IAA? Figure 7 shows that Ler, and especially Col are much less sensitive to exogenous IAA than Cvi: within the tested range of concentrations, Col hardly responds, whereas Cvi shows a $30 \%$ decrease in root length already at the lowest concentration tested. Ler showed an intermediate behaviour.

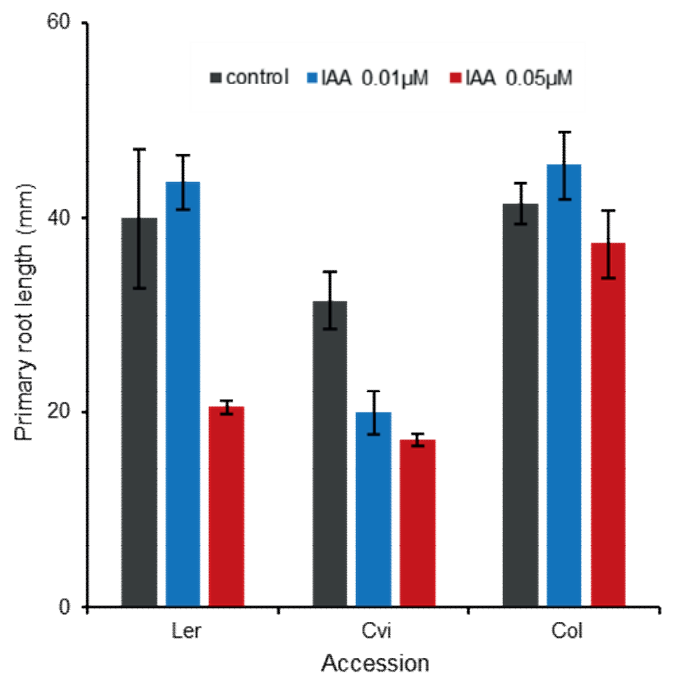

Figure 7. Effect of indole-3-acetic acid (IAA) on primary root length in Arabidopsis seedlings. Plants were grown on vertical plates with half strength of Murashige and Skoog media, containing IAA. Primary root lengths were measured after $10 \mathrm{~d}$ of culturing.

\section{Quantitative changes in hormone contents during mature root development}

Quantitative changes and profiling of phytohormones in organs/tissues have been reported in some plants (Kanno et al., 2010; Powell et al., 2013; Taylor and vanStaden, 1997). In Arabidopsis, most studies on hormone quantification have focused on young seedlings, and hence data on dynamic changes during rapid root growth in later stages are missing. Information on quantitative changes in hormones, if occurring, may help to understand their biochemical and functional roles in root growth. Therefore, we measured quantitative changes in phytohormones in the roots of two representative accessions (Col-0, Ler-0), which showed vigorous vegetative growth during five weeks (23-58 days, before transition to flowering) (Figure S3). 
Overall, each phytohormone showed similar time-course patterns in both accessions (Figure 8), although there were some minor differences. Auxin level decreased between 30 and 58 days in both accessions, with a $40 \%$ decrease in Col- 0 and $32 \%$ in Ler. ABA sharply decreased in both accessions from 23 days to around 40 days. Thereafter, ABA levels became below the detection limit. Levels of CK free bases were fairly stable for the whole period, although $\mathrm{tZ}$ in Col-0 slightly decreased (22\%) between 23 and 44 days. CK ribosides (iPR, tZR, DZR) did not change much in both lines, while levels of glucosides $(\mathrm{tZ7G}, \mathrm{tZ}(\mathrm{O}, 9) \mathrm{G})$ gradually increased after 37 days: $\mathrm{tZ} 7 \mathrm{G}, 73 \%$ in $\mathrm{Col}-0$ and $156 \%$ in Ler; $\mathrm{tZ}(\mathrm{O}, 9) \mathrm{G}, 112 \%$ in Col-0 and $274 \%$ in Ler. Levels of dihydrozeatin (DZ) and gibberellins, which could be measured in 23-day-old roots, were below the detection limit after 30 days.
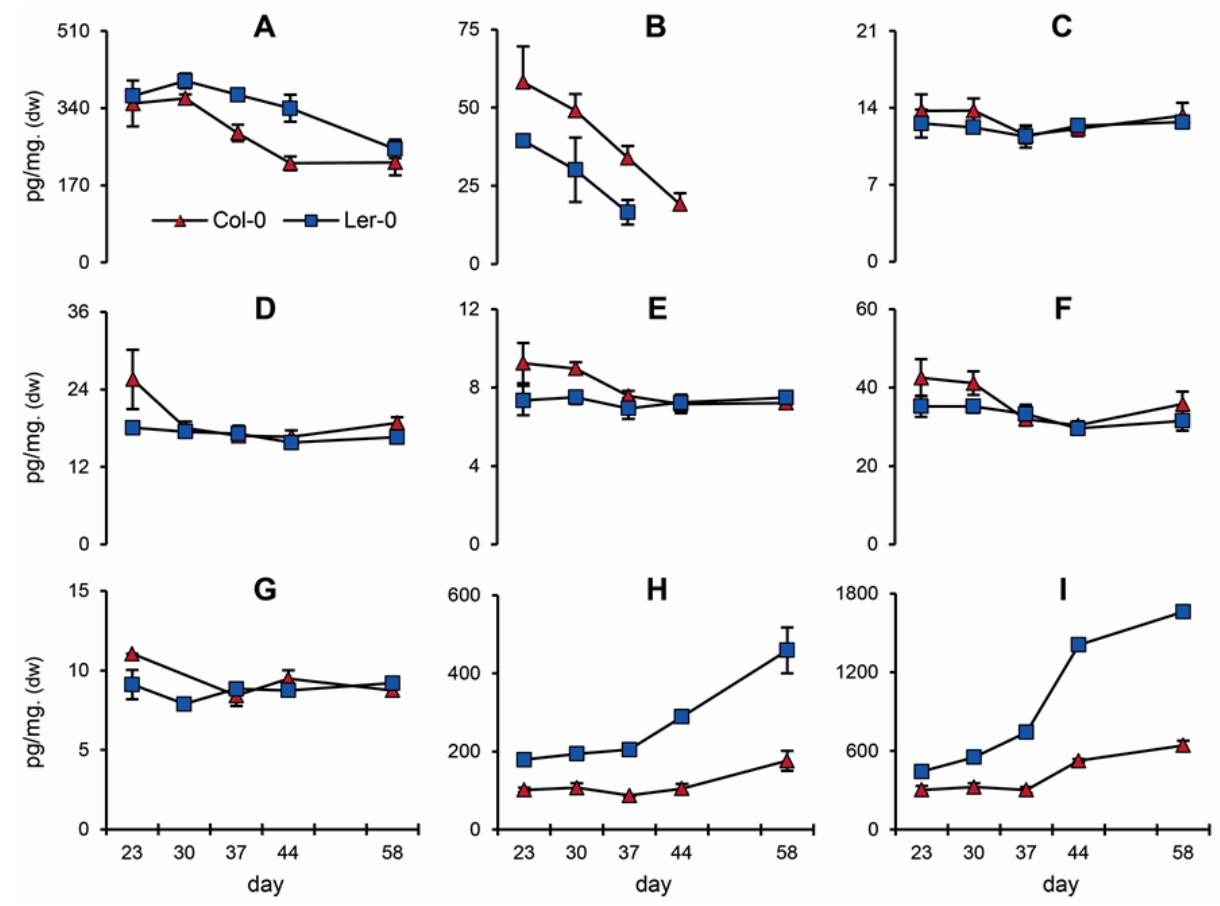

Figure 8. Temporal changes of hormone levels in Arabidopsis roots during mature root development. (A) Indole-3-acetic acid (IAA). (B) Abscisic acid (ABA). (C) Isopentenyl adenine (iP). (D) Isopentenyl adenine riboside (iPR). (E) Trans-zeatin (tZ). (F) Trans-zeatin riboside tZR). (G) Dihydrozeatin riboside (DZR). (H) Trans-zeatin-7-glucoside (tZ7G). (I) Trans-zeatin-(O and 9)-glucosides $(\mathrm{tZ}(\mathrm{O}, 9) \mathrm{G})$. Red triangles and blue squares indicate Columbia- 0 and Ler- 0 , respectively. Unit of Y-axes is $\mathrm{pg} \mathrm{mg}^{-1}$ in dry weight commonly. Vertical bars on markers indicate standard errors $(n=4)$. Data of $51^{\text {st }} \mathrm{d}$ were missed. ABA levels were below the detection limit after $37 \mathrm{~d}$ for Ler- 0 and $44 \mathrm{~d}$ for Col- 0 . 


\section{Discussion}

Roots are vital for plant growth and survival, and their development should adequately respond to environmental cues. Hormones are supposed to be essential in this process, transducing the external signals to internal ones. Different accessions of one species may have adapted to a specific local environment, thus resulting in natural variation within the species for root-related traits. We tried to answer the question whether, and to what extent, endogenous hormones are involved in such adaptations, by determining endogenous levels of a series of hormones, and how they correlate with root phenotypic traits. Since the root architecture in later stages of the development plays an important role in the adaptation to the environment, we decided to focus on mature plants, rather than seedlings that have been investigated in many other studies related to root architecture (Slovak et al., 2014; Zhu et al., 2011).

\section{Evaluation of mature RSA requires proper root phenotypic traits}

Variation in phenotypic traits in 23-day-old mature roots was large and different for each trait, similar as found in growth-related traits in the shoot (El-Lithy et al., 2004). The larger variation in LRL traits, as compared to LRN, for example, shows that Arabidopsis displays a smaller genetic variation for lateral root numbers than for length. The obvious differences in lateral root lengths, despite the homogeneous hydroponic culture, suggest that phenotypic plasticity of Arabidopsis root system to environmental constraints. e.g., drought, may be considerable. A large variation was also observed in root weight as was shown earlier for young seedlings (Clark et al., 2013; Slovak et al., 2014). However, PRL and LRD, important traits to evaluate phenotypes of seedling roots, were less variable in the present study, suggesting that phenotypic traits of interest in mature roots should be carefully chosen for proper description and quantification.

\section{Arabidopsis roots show homeostasis for phytohormone levels}

The natural variation in the levels of hormones in the present study was surprisingly low, differences being less than two-folds between extremes, except for $\mathrm{GA}_{9}$. These conserved levels of hormones are in stark contrast with other studies on primary and secondary metabolites in plants. The concentrations of flavonoids in seeds of 41 Arabidopsis ecotypes showed large variation: 39-fold for quercetin-3-rhamnoside and 9.6-fold for biflavonols (Routaboul et al., 2012). There was a twenty-fold difference for total aliphatic glucosinolate levels among leaves of 39 Arabidopsis ecotypes (Kliebenstein et al., 2001b). Also in a metabolite study in tomato, the variation in levels of primary and secondary metabolites was large, for instance, tocopherol contents showed 10-fold differences between extreme lines (Sauvage et al., 2014). 
Earlier reports described large natural variation in the response to hormones and stresses, as detected in transcriptome studies (Delker et al., 2010; Sofo et al., 2013). The limited variation in hormone levels, including biologically inactive conjugates and other products of inactivation that we found in the present study, would imply that levels of phytohormones are controlled by sophisticated homeostasis mechanisms, presumably involving conjugation, oxidation, transport and synthesis. More fine-tuned homeostatic control can be found for active hormone compounds, e.g., CK free bases, as described in the present study, which are regulated in coordination with complex signalling mechanisms in non-linear pathways (Vanstraelen and Benkova, 2012).

It should be noted, however, that hormone levels in our study were determined in a whole-root extract, thus not including information on possible differential concentrations, which have been founded at the cellular or tissue level (Antoniadi et al., 2015; Bielach et al., 2012). Though incomplete, the present data are still valuable to advance our understanding of the natural variation in homeostatic regulation of phytohormones between active forms and intermediates in Arabidopsis roots.

The variation in auxin level $( \pm 25 \%)$ in our study can be a consequence of two features: genetic polymorphism for genes involved in auxin metabolism, and variation for transporters, such as PINs/ABCBs and AUXs (Kramer and Bennett, 2006; Petersson et al., 2009), resulting in local differences of levels in the root system. The larger variation in the level of GA9 may be a feature of an inactive GA as a precursor for the biologically active $\mathrm{GA}_{4}$ (Hedden and Thomas, 2012) but more quantitative information for other GA species is required to understand the natural variation for this large class of hormones, which are relatively more abundant in leaves than in roots (Nam et al., 2017).

\section{Correlation analysis between hormones and RSA may help to understand the hormonal cross-} talk that determines root architecture

Positive correlations of auxin level to TRTN and TRL were consistent with previous findings in seedlings. This would imply that the local maxima for auxin in the root apices, as observed in seedlings, are also present in mature roots. In our study, RSA seems to be controlled mainly by antagonistic relationships between IAA on the one hand, and CKs (tZ, iP), ABA and GA, on the other hand (Figure 6). Cross-talks between CK signalling and PIN-FORMED (PIN) expression occur in the early embryonic root development (Bishopp et al., 2011; Muller and Sheen, 2008). The underlying mechanism may be that $\mathrm{CK}$ suppresses the expression of PIN1, thus depleting polarly localized PIN proteins at the basal membrane, consequently decreasing directional auxin flow (Marhavy et al., 2014). Auxin-CK balance also regulates the root meristem size in Arabidopsis, through triggering of CK signalling, thus affecting root biomass (Dello Ioio et al., 2008; Ruzicka et al., 2009). 
Another antagonistic hormone interaction for mature root development that showed up in the PCA was that between GA9 and auxin (Figure 6). According to Moubayidin et al. (2010), gibberellin $\left(\mathrm{GA}_{3}\right)$ induces PIN expression via repression of ARABIDOPSIS RESPONSE REGULATOR 1 (ARRI) and IAA3/SHY2 transcription but attenuates CK activity. This positive regulation of auxin by GA conflicts with the present study that $\mathrm{GA}_{9}$ clustered with $\mathrm{tZ}$ rather than with IAA. This discrepancy may be due to the large differences between different GAs, with the biologically active GAs $\left(\mathrm{GA}_{3}\right.$, $\mathrm{GA}_{4}$ ), showing opposite behaviour from the inactive ones such as $\mathrm{GA}_{9}$, similar to the opposite behaviour between $\mathrm{CK}$ ribosides (iPR, tZR) and $\mathrm{tZ}$ as seen in the PCA (Figure 6).

In the present study, clustering of $\mathrm{ABA}$ with $\mathrm{tZ}$ could be compatible with the results presented by Shkolnik-Inbar and Bar-Zvi (2010), showing that LR growth can be altered by the ABSCISIC ACID INSENSITIVE 4 (ABI4) transcription factor, whose expression is enhanced by ABA and CK, and reduces polar auxin transport, finally causing inhibition of LR formation. Overall, these possible hormonal cross-talks seem also compatible with previous results, showing comparable phenotypic plasticity in wild-type (Col-0) and a number of Arabidopsis mutants (axr4, abi4, crel) under various treatments (IAA, ABA, CK) (Ristova et al., 2013).

The experiment with exogenously applied hormone (Figure 7) shows the importance of hormone-sensitivity in controlling root growth. However, the various significant positive and negative correlations between hormone levels and root traits (Table 2) also indicate a prominent role for the endogenous hormone levels themselves.

\section{Changes in hormone concentrations during root growth may reflect changes in spatial distribution and localization}

Based on quantitative changes during root development (Figure 8), phytohormones can be divided into three groups: decreasing (IAA, ABA), constant (tZ, tZR, iP, iPR) and increasing (tZ7G, tZ(O, 9)G). Auxin levels show local maxima in root apices (Grieneisen et al., 2007, Petersson et al. 2009); thus the gradual decrease in the auxin level during root development and elongation likely reflects a dilution effect, with relatively less apices as compared to total root mass in older roots. Hydroponic culture provides a homogenous environment for roots that may normally be sensitive to dryness in the rhizosphere, inducing local ABA accumulation as observed in potatoes (Puertolas et al., 2015). In maize, younger roots have higher ABA levels than older roots (Zhang and Tardieu, 1996), which is consistent with the decrease in ABA levels during root maturation in the present study.

In a current model of CK biosynthesis in plants, ribosides and free bases are synthesized through two paths; one is from tZRMP to tZR and finally to tZ, including two enzymatic steps and the other is from tZRMP directly to tZ catalysed by LONELY GUY (LOG) without tZR as an intermediate (Kamada-Nobusada and Sakakibara 2009). Studies using multiple mutants and overexpression lines 
for $L O G$ genes, show that the levels of CK ribosides and free bases are cumulatively affected by LOG homologues, each of them having a small additive effect (Kuroha et al. 2009, Tokunaga et al. 2012). Both non-linear biosynthetic pathways and polygenic regulation for a given enzymatic conversion may support long-term homeostasis of ribosides and free bases as found in the present study of Arabidopsis roots.

CK $\mathrm{N}$-glucosides are products of a linear metabolic pathway and are synthesized irreversibly from free bases by $\mathrm{N}$-glucosyltransferases, followed by $\mathrm{CK}$ oxidases/dehydrogenases $(C K X)$ for degradation. The significant positive correlation of $\mathrm{tZ7G}$ with RFW and TRL in the 23-day-old roots and the accumulation of $\mathrm{tZ}(7,9) \mathrm{G}$ during rapid root growth are consistent with the findings of Kollmer et al. (2014) that decreased levels of $N$-glucosides, due to overexpression of $C K X 7$, result in an early termination of the primary root growth, complete suppression of lateral root initiation and aberration of root vascular development. It is yet unknown how the accumulation of CK $N$-glucosides is controlled, their levels depending on two enzymatic reactions, between $\mathrm{N}$-glucosylation and oxidation. Presumably, CK $N$-glucosides may have a physiological role in Arabidopsis root growth, different from $O$-glucosides that are relevant for homeostatic regulation of biologically active CK.

In summary, here we report that endogenous hormone levels in roots of natural accessions of Arabidopsis are maintained within a narrow range of concentrations. Complex RSA in mature plants, consisting of multiple-order lateral roots, should be studied using appropriate phenotypic traits, since the use of only two components - the primary and the lateral roots - structuring young roots, is not suitable to effectively describe mature root systems. In correlation analyses we showed that some phenotypic traits in mature roots can be explained by hormone cross-talk. Since natural variation is an important premise to unravel genetic elements through quantitative traits analyses (Koornneef et al., 2004), this study suggests that quantitative trait loci (QTL) analysis for phytohormone levels is feasible, using mapping populations derived from divergent accessions, for example, Ler and Cvi (Figure S4). This way, the molecular mechanisms by which root architecture in plants is determined, will be further unravelled.

\section{Acknowledgements}

We thank the Provincial Government of Gyeongsangbuk-do in South Korea for overseas-training program to Sangseok Lee, Dr. Kristyna Flokova for advice for hormone analysis and Dr. Harro Bouwmeester for proof-reading the manuscript. 


\section{[Supplementary Information]}

Table S1. Summary of multiple reaction monitor (MRM) transitions used for hormone quantification in ESI-TQ mass spectrometer.

\begin{tabular}{|c|c|c|c|c|c|c|c|c|c|c|c|c|}
\hline Analytes & $\begin{array}{c}\text { UPLC } \\
\text { Gradient }\end{array}$ & RT & ESI & $\begin{array}{c}\text { MRM } \\
\text { Function }\end{array}$ & $\begin{array}{l}\text { Transition } \\
(\mathrm{m} / \mathrm{z})\end{array}$ & $\begin{array}{c}\text { Cone } \\
\text { voltage }\end{array}$ & $\begin{array}{l}\text { Collision } \\
\text { energy }\end{array}$ & $\begin{array}{l}\text { Internal } \\
\text { standard }\end{array}$ & RT & $\begin{array}{l}\text { Transition } \\
(\mathrm{m} / \mathrm{z})\end{array}$ & $\begin{array}{c}\text { Cone } \\
\text { voltage }\end{array}$ & $\begin{array}{c}\text { Collision } \\
\text { energy }\end{array}$ \\
\hline $\mathrm{tZ}$ & 1 & 3.78 & + & 2 & $220.1>136.1$ & 20 & 18 & {$\left[{ }^{2} \mathrm{H}_{6}\right] \mathrm{tZ}$} & 3.75 & $225.1>136.9$ & 20 & 18 \\
\hline tZR & 1 & 5.04 & + & 3 & $352.1>136.1$ & 28 & 20 & {$\left[{ }^{2} \mathrm{H}_{5}\right] \mathrm{tZR}$} & 5.01 & $357.1>136.9$ & 28 & 20 \\
\hline iP & 1 & 6.15 & + & 1 & $204.1>136.1$ & 20 & 18 & {$\left[{ }^{2} \mathrm{H}_{6}\right] \mathrm{iP}$} & 6.11 & $210.1>137.1$ & 20 & 18 \\
\hline iPR & 1 & 7.60 & + & 1 & $336.1>204.1$ & 28 & 18 & {$\left[{ }^{2} \mathrm{H}_{6}\right] \mathrm{iPR}$} & 7.55 & $342.2>210.2$ & 28 & 18 \\
\hline$c Z$ & 1 & 4.01 & + & 2 & $220.1>136.1$ & 20 & 18 & {$\left[{ }^{2} \mathrm{H}_{6}\right] \mathrm{tZ}$} & 3.75 & $225.1>136.9$ & 20 & 18 \\
\hline CZR & 1 & 5.31 & + & 3 & $352.1>136.1$ & 28 & 16 & {$\left[{ }^{2} \mathrm{H}_{5}\right] \mathrm{tZR}$} & 5.01 & $357.1>136.9$ & 28 & 20 \\
\hline tZOG & 1 & 3.84 & + & 2 & $382.1>220.1$ & 30 & 16 & {$\left[{ }^{2} \mathrm{H}_{5}\right] \mathrm{tZ} 9 \mathrm{G}$} & 4.02 & $387.2>225.2$ & 30 & 20 \\
\hline $\mathrm{tZ7G}$ & 1 & 3.86 & + & 2 & $382.1>220.1$ & 30 & 20 & {$\left[{ }^{2} \mathrm{H}_{5}\right]$ tZ9G } & 4.02 & $387.2>225.2$ & 30 & 20 \\
\hline tZ9G & 1 & 4.04 & + & 2 & $382.1>220.1$ & 30 & 20 & {$\left[{ }^{2} \mathrm{H}_{5}\right] \mathrm{tZ9G}$} & 4.02 & $387.2>225.2$ & 30 & 20 \\
\hline DZ & 1 & 3.95 & + & 2 & $222.1>136.1$ & 20 & 18 & {$\left[{ }^{2} \mathrm{H}_{3}\right] \mathrm{DZ}$} & 3.91 & $225.1>136.1$ & 20 & 18 \\
\hline DZR & 1 & 5.10 & + & 3 & $354.1>136.1$ & 28 & 22 & {$\left[{ }^{2} \mathrm{H}_{3}\right] \mathrm{DZR}$} & 5.06 & $357.2>149.1$ & 28 & 22 \\
\hline DZ7G & 1 & 4.07 & + & 2 & $384.1>136.1$ & 30 & 20 & & & & & \\
\hline DZ9G & 1 & 4.17 & + & 2 & $384.1>136.1$ & 30 & 20 & & & & & \\
\hline DZROG & 1 & 5.12 & + & 3 & $516.1>222.1$ & 34 & 20 & & & & & \\
\hline tZRMP & 1 & 3.58 & + & 2 & $432.1>220.1$ & 34 & 22 & & & & & \\
\hline tZROG & 1 & 4.94 & + & 3 & $514.1>382.2$ & 34 & 22 & & & & & \\
\hline $\mathrm{mT}$ & 1 & 4.92 & + & 3 & $242.1>77.0$ & 20 & 18 & & & & & \\
\hline mTR & 1 & 6.45 & + & 1 & $374.1>242.1$ & 30 & 20 & & & & & \\
\hline oT & 1 & 5.94 & + & 1 & $242.1>136.1$ & 20 & 18 & & & & & \\
\hline OTR & 1 & 7.55 & + & 1 & $374.1>242.1$ & 28 & 20 & & & & & \\
\hline IAA & 2 & 4.15 & + & 1 & $176.1>130.1$ & 18 & 16 & {$\left[{ }^{13} \mathrm{C}_{6}\right]$ IAA } & 4.14 & $182.1>136.1$ & 18 & 16 \\
\hline $\mathrm{ABA}$ & 2 & 4.80 & - & 5 & $263.1>219.1$ & 18 & 14 & {$\left[{ }^{2} \mathrm{H}_{6}\right] \mathrm{ABA}$} & 4.77 & $269.1>225.1$ & 18 & 14 \\
\hline $\mathrm{GA}_{1}$ & 2 & 3.67 & + & 4 & $349.2>285.2$ & 20 & 16 & {$\left[{ }^{2} \mathrm{H}_{2}\right] \mathrm{GA}_{1}$} & 3.65 & $351.2>287.2$ & 20 & 16 \\
\hline $\mathrm{GA}_{3}$ & 2 & 3.61 & - & 7 & $345.2>239.2$ & 28 & 14 & {$\left[{ }^{2} \mathrm{H}_{2}\right] \mathrm{GA}_{3}$} & 3.60 & $347.2>241.2$ & 28 & 14 \\
\hline $\mathrm{GA}_{4}$ & 2 & 6.15 & - & 6 & $331.2>257.2$ & 28 & 14 & {$\left[{ }^{2} \mathrm{H}_{2}\right] \mathrm{GA}_{4}$} & 6.13 & $333.2>259.2$ & 28 & 14 \\
\hline $\mathrm{GA}_{5}$ & 2 & 4.83 & + & 3 & $331.2>285.2$ & 12 & 10 & & & & & \\
\hline $\mathrm{GA}_{7}$ & 2 & 6.06 & - & 6 & $329.2>223.2$ & 26 & 14 & {$\left[{ }^{2} \mathrm{H}_{2}\right] \mathrm{GA}_{7}$} & 6.04 & $331.2>225.2$ & 26 & 14 \\
\hline $\mathrm{GA}_{8}$ & 2 & 2.69 & + & 4 & $365.2>301.2$ & 14 & 10 & & & & & \\
\hline $\mathrm{GA}_{9}$ & 2 & 7.18 & + & 2 & $317.2>271.2$ & 16 & 14 & {$\left[{ }^{2} \mathrm{H}_{2}\right] \mathrm{GA}_{4}$} & 6.13 & $333.2>259.2$ & 28 & 14 \\
\hline $\mathrm{GA}_{19}$ & 2 & 4.80 & + & 3 & $363.2>299.2$ & 14 & 12 & & & & & \\
\hline $\mathrm{GA}_{20}$ & 2 & 4.95 & + & 3 & $333.2>287.2$ & 18 & 12 & {$\left[{ }^{2} \mathrm{H}_{2}\right] \mathrm{GA}_{20}$} & 4.93 & $335.2>289.2$ & 18 & 12 \\
\hline $\mathrm{GA}_{44}$ & 2 & 5.05 & + & 3 & $347.2>301.2$ & 22 & 14 & & & & & \\
\hline $\mathrm{GA}_{53}$ & 2 & 5.65 & + & 6 & $347.2>329.2$ & 48 & 22 & & & & & \\
\hline
\end{tabular}

Table S2. Hormone levels in 23-d-old roots of 13 Arabidopsis accessions (unit: pg mg-1 .dry weight).

\begin{tabular}{|c|c|c|c|c|c|c|c|c|c|c|c|c|c|c|c|c|}
\hline & IAA & $A B A$ & & iP & & iPR & & $c Z$ & & tZ & & tZR & tZ7G & $\mathrm{tZ}(0,9) \mathrm{G}$ & & $A_{9}$ \\
\hline$A n-1$ & $385.8 \pm 11.8$ & 22.3 & 14.1 & \pm 0.5 & 10.3 & \pm 0.9 & 60.2 & \pm 2.6 & 21.5 & \pm 0.6 & 50.0 & \pm 9.2 & $194.3 \pm 10.4$ & $307.8 \pm 7.9$ & 107.6 & \pm 28.9 \\
\hline Bay-1 & $504.9 \pm 14.2$ & $17.5 \pm 2.0$ & 15.1 & \pm 0.1 & 21.8 & \pm 0.8 & 55.7 & \pm 1.4 & 21.2 & \pm 0.4 & 64.4 & \pm 6.5 & $148.4 \pm 10.4$ & $240.5 \pm 16.7$ & 108.9 & \pm 14.1 \\
\hline Bor-4 & $396.4 \pm 19.4$ & $16.2 \pm 1.1$ & 14.1 & \pm 0.3 & 19.4 & \pm 1.2 & 53.9 & \pm 4.4 & 20.9 & \pm 0.4 & 44.5 & \pm 2.9 & $178.6 \pm 11.1$ & $269.4 \pm 13.2$ & 68.4 & \pm 2.5 \\
\hline Bur-0 & $318.2 \pm 35.6$ & $16.0 \pm 2.6$ & 14.3 & \pm 0.2 & 13.0 & \pm 0.8 & 58.1 & \pm 1.4 & 20.4 & \pm 0.3 & 46.6 & \pm 2.5 & $199.2 \pm 13.7$ & $287.8 \pm 26.8$ & 209.0 & \pm 56.0 \\
\hline Col-0 & $387.6 \pm 2.2$ & $15.5 \pm 0.6$ & 13.8 & \pm 0.4 & 13.7 & \pm 2.1 & 53.2 & \pm 6.9 & 22.3 & \pm 0.2 & 50.8 & \pm 4.3 & $140.7 \pm 17.9$ & $229.2 \pm 10.5$ & 105.2 & \pm 23.2 \\
\hline Cvi-0 & $377.7 \pm 18.7$ & $28.0 \pm 2.0$ & 13.4 & \pm 0.9 & 10.0 & \pm 1.0 & 58.7 & \pm 3.7 & 32.0 & \pm 4.0 & 37.1 & \pm 5.9 & $138.2 \pm 10.0$ & $212.5 \pm 10.5$ & 142.9 & \pm 23.1 \\
\hline Est-1 & $468.6 \pm 6.1$ & $16.3 \pm 1.1$ & 13.8 & \pm 0.3 & 13.8 & \pm 1.4 & 52.1 & \pm 4.3 & 21.1 & \pm 0.6 & 41.8 & \pm 4.2 & $164.9 \pm 6.6$ & $300.5 \pm 18.4$ & 139.8 & \pm 11.4 \\
\hline Fei-0 & $410.9 \pm 14.2$ & $15.2 \pm 1.1$ & 14.6 & \pm 0.1 & 12.0 & \pm 0.5 & 46.7 & \pm 5.4 & 21.4 & \pm 0.7 & 41.5 & \pm 0.3 & $145.7 \pm 4.8$ & $265.4 \pm 26.7$ & 121.5 & \pm 19.1 \\
\hline Ler-0 & $457.6 \pm 6.0$ & $16.6 \pm 1.0$ & 15.2 & \pm 0.3 & 17.0 & \pm 2.3 & 74.3 & \pm 2.8 & 21.5 & \pm 0.4 & 48.6 & \pm 2.8 & $191.4 \pm 11.8$ & $305.4 \pm 9.8$ & 95.0 & \pm 23.0 \\
\hline RRS-7 & $329.0 \pm 4.1$ & $16.0 \pm 2.1$ & 15.4 & \pm 0.6 & 9.5 & \pm 1.9 & 63.4 & \pm 5.2 & 24.2 & \pm 1.2 & 47.1 & \pm 4.4 & $212.7 \pm 26.0$ & $314.0 \pm 23.7$ & 168.6 & \pm 28.8 \\
\hline Sha-0 & $381.1 \pm 15.7$ & $20.4 \pm 2.3$ & 15.1 & \pm 0.3 & 18.1 & \pm 1.0 & 87.8 & \pm 2.0 & 23.2 & \pm 0.6 & 78.8 & \pm 10.6 & $192.5 \pm 22.1$ & $339.7 \pm 13.8$ & 178.3 & \pm 5.6 \\
\hline Ts-1 & $377.2 \pm 28.7$ & $17.0 \pm 2.1$ & 15.7 & \pm 0.5 & 11.6 & \pm 0.8 & 61.1 & \pm 5.9 & 22.0 & \pm 0.8 & 42.1 & \pm 3.4 & $142.0 \pm 16.1$ & $244.8 \pm 15.3$ & 172.3 & \pm 30.0 \\
\hline Tsu-0 & $360.5 \pm 8.9$ & $20.1 \pm 4.0$ & 14.9 & \pm 0.2 & 16.5 & \pm 1.6 & 90.9 & \pm 1.9 & 22.0 & \pm 0.2 & 61.1 & \pm 2.0 & $208.5 \pm 9.8$ & $307.9 \pm 26.4$ & 104.9 & \pm 11.7 \\
\hline Median & 391.8 & 16.4 & 14.7 & & 13.5 & & 58.7 & & 21.9 & & 46.8 & & 172.1 & 286.8 & 120.5 & \\
\hline Mean & 402.7 & 17.8 & 14.6 & & 14.7 & & 63.1 & & 22.2 & & 50.7 & & 174.1 & 280.7 & 126.7 & \\
\hline S.D & 57.6 & 4.3 & 0.9 & & 4.4 & & 14.8 & & 2.6 & & 13.7 & & 34.1 & 45.1 & 49.8 & \\
\hline
\end{tabular}


Table S3. Contribution of variables (hormones and root phenotypic traits) on PCA (unit: \%).

\begin{tabular}{|c|c|c|c|c|c|}
\hline variables & PC1 & PC2 & $\mathrm{PC} 3$ & PC4 & PC5 \\
\hline RFW & 5.07 & 0.68 & 4.07 & 0.04 & 2.45 \\
\hline MRUN & 4.20 & 4.48 & 2.45 & 0.32 & 1.28 \\
\hline TRL & 7.26 & 0.50 & 0.95 & 0.48 & 1.36 \\
\hline TRTN & 6.16 & 1.54 & 3.03 & 0.63 & 0.49 \\
\hline TRD & 2.34 & 0.12 & 12.94 & 0.20 & 4.89 \\
\hline $\mathrm{RL}$ & 3.50 & 5.66 & 3.33 & 0.83 & 2.72 \\
\hline LRL & 7.77 & 0.08 & 0.07 & 0.90 & 0.41 \\
\hline LRN & 4.79 & 0.15 & 8.40 & 1.11 & 0.35 \\
\hline LRD & 1.79 & 8.45 & 4.41 & 0.26 & 4.55 \\
\hline $2^{\prime}-\mathrm{LRL}$ & 5.55 & 2.50 & 2.88 & 0.20 & 1.89 \\
\hline $2^{\prime}-\mathrm{LRN}$ & 5.43 & 3.18 & 1.13 & 2.05 & 0.42 \\
\hline $2^{\prime}-$ LRD & 0.46 & 2.71 & 10.31 & 5.54 & 1.98 \\
\hline LRL-1Q & 6.07 & 1.46 & 0.18 & 0.05 & 6.85 \\
\hline LRL-2Q & 7.06 & 0.11 & 0.56 & 2.29 & 0.01 \\
\hline LRL-3Q & 3.90 & 6.85 & 0.38 & 3.24 & 1.34 \\
\hline LRL-4Q & 0.29 & 13.02 & 0.73 & 0.03 & 2.42 \\
\hline LRN-1Q & 2.27 & 1.48 & 3.26 & 11.65 & 0.01 \\
\hline LRN-2Q & 4.38 & 0.73 & 2.61 & 5.92 & 0.31 \\
\hline LRN-3Q & 4.32 & 0.37 & 10.08 & 0.10 & 0.01 \\
\hline LRN-4Q & 0.79 & 8.05 & 5.85 & 5.93 & 0.30 \\
\hline IAA & 0.36 & 3.10 & 0.27 & 4.09 & 25.55 \\
\hline$A B A$ & 0.01 & 2.21 & 0.87 & 14.09 & 10.86 \\
\hline iP & 0.19 & 3.37 & 2.54 & 8.51 & 5.09 \\
\hline iPR & 3.32 & 0.28 & 5.61 & 0.70 & 10.00 \\
\hline$c z$ & 2.34 & 10.06 & 1.13 & 0.39 & 0.97 \\
\hline tz & 1.25 & 2.28 & 3.59 & 13.72 & 3.06 \\
\hline tZR & 3.60 & 5.27 & 1.82 & 0.01 & 3.08 \\
\hline $\mathrm{tZ7G}$ & 2.99 & 1.76 & 0.08 & 4.07 & 7.20 \\
\hline $\mathrm{tZ}(0,9) \mathrm{G}$ & 1.99 & 3.68 & 0.03 & 3.71 & 0.04 \\
\hline $\mathrm{GA}_{9}$ & 0.41 & 5.76 & 6.31 & 8.80 & 0.01 \\
\hline
\end{tabular}



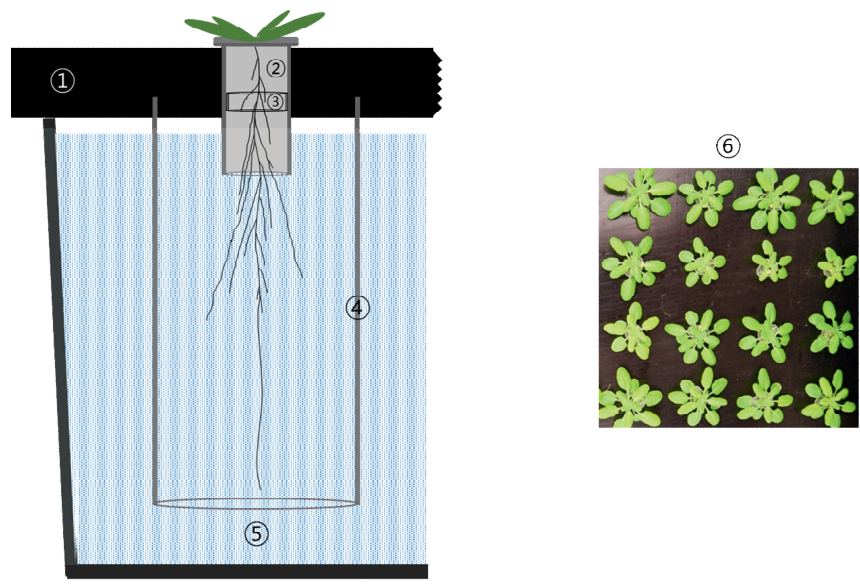

Figure S1. Illustration of hydroponic culture. The black plate (1) is placed and covered on the hydroponic tank. A sprout on the top of holder-tube $(0.5 \mathrm{ml})$ (2) filled with $0.5 \%$ agar in half of Hoagland's nutrient solution, grows and develops root system in the hydroponic tank. A small ring (3) inside the tube was tightly placed to prevent agar medium from being slipped down out of the tube during culture, with no physical hindrance for root development. In order to avoid roots to be entangled with roots of neighbour plants, a polypropylene column (diameter $3 \mathrm{~cm}$, height $5.5 \mathrm{~cm}$ ) (4) was equipped underneath the black plate, allowing nutrient solution and roots not to be blocked on the bottom (5). Right picture (6) shows how upper shoots settle and grow on the black plate.

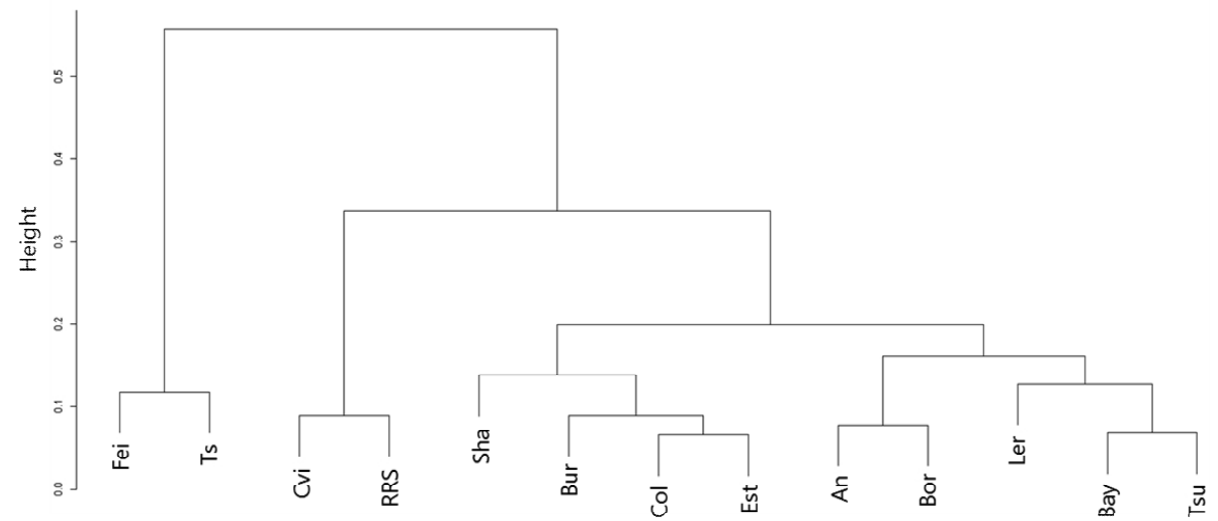

Figure S2. Hierarchical cluster analysis of RSA traits in 13 Arabidopsis accessions. 


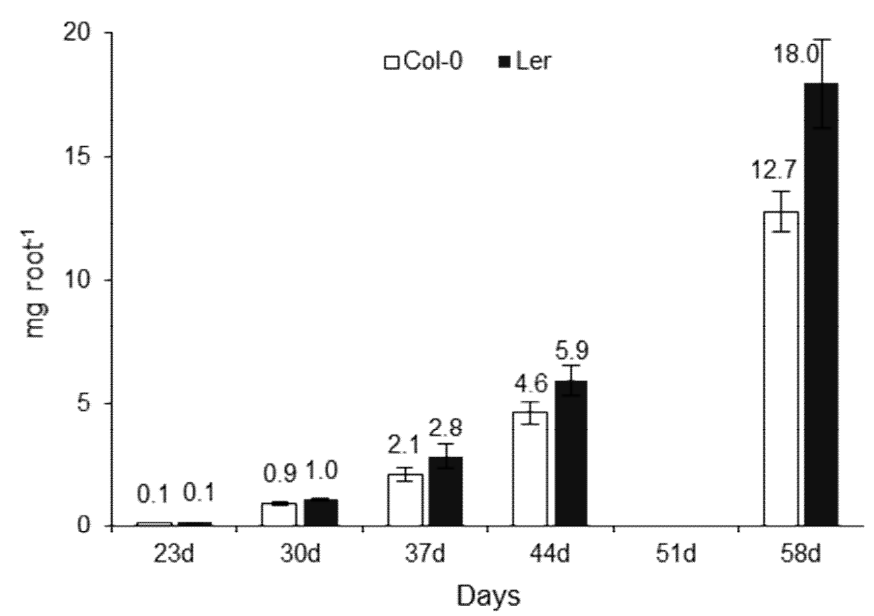

Figure S3. Dry root weights of Col-0 and Ler-0 during 5 weeks of root development. Data of $51^{\text {th }}$ day was missed.

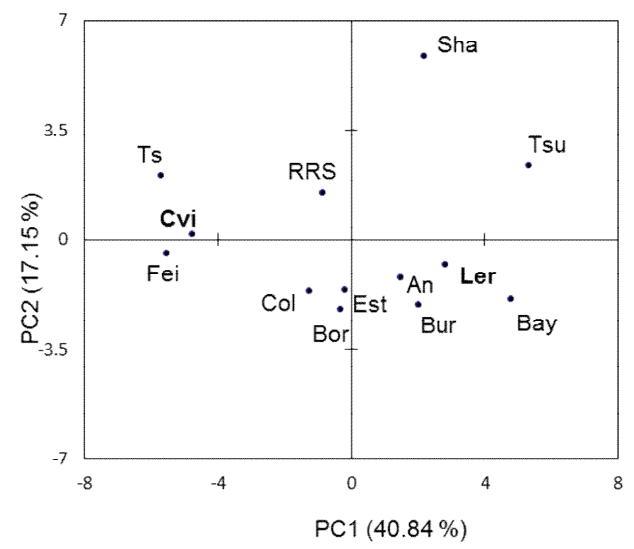

Figure S4. Re-partition of 13 Arabidopsis accessions in PCA of hormone levels and RSA traits. 



\section{Chapter 4}

\section{Quantitative trait loci analysis of hormone levels in Arabidopsis roots}

Sangseok Lee, Lidiya I. Sergeeva and Dick Vreugdenhil 


\begin{abstract}
Quantitative trait loci (QTL) analyses for five groups of hormones, including cytokinins in Arabidopsis roots were performed using recombinant inbred lines (Ler $\times$ Cvi). Significant QTLs were detected for cytokinins, jasmonic acid and salicylic acid. Separate analysis of two sub-populations, viz., vegetative and flowering plants revealed that many of the QTLs were development-specific. Using near-isogenic lines, several significant QTLs were confirmed; three co-localized QTL regions were responsible for determining several cytokinin metabolites. Using a knock-out plant, a functional role of zeatin $\mathrm{N}$-glucosyltransferase gene (UGT76C2) underlying a large-effect QTL for levels of tZ$\mathrm{N}$-glucosides and tZRMP was evaluated in the metabolism of cytokinins. Pleotropic effects of this gene were found for cytokinin levels in both roots and leaves, but significant changes of morphological traits were observed only in roots. Hormone QTL analysis reveals developmentspecific and organ-dependent aspects of the regulation of plant hormone content and metabolism.
\end{abstract}

Key words: Arabidopsis thaliana, quantitative trait loci (QTLs), plant hormones, recombinant inbred lines (RILs), near-isogenic lines (NILs) 


\section{Introduction}

Plant hormones are naturally occurring organic substances that influence complex processes in plant development at extremely low concentrations. Hormone levels vary during plant development, between organs, e.g., roots and shoots, and between vegetative and flowering stages (Alvim et al., 1978; Novakova et al., 2005; Powell et al., 2013). Within a species, hormone concentrations may also vary between different varieties (Powell et al., 2013). Such variations of plant hormones are obviously determined by genes involved in anabolic and catabolic pathways, transport facilitators and/or signalling components (Mashiguchi et al., 2011; Spiess et al., 2014).

Naturally occurring variations among different accessions for a particular trait are due to allelic diversity and insertion/deletion (INDELs) of bases including gene duplication in genomes in a species (Cao et al., 2011). Even allelic difference at a single locus can contribute to pleiotropic differences in growth and fitness (Todesco et al., 2010). Such allelic variations may induce changes in metabolic profiles, as shown by Chayut et al. (2015); allelic variation of the melon's Or gene (CmOr) resulted in an increase of beta-carotene accumulation in melon fruit. Hence, the quantitative variation of plant hormones within a species is also expected to be due to genetic heterogeneity.

The quantitative variation of hormone levels can be considered as a complex phenotypic trait, determined by multiple loci, which may interact with each other. Quantitative trait loci (QTL) analysis has been used to identify genomic regions responsible for polygenic traits. In comparison with direct-mutagenesis approach, QLT analysis is more likely to identify genes encoding regulatory proteins or rate-determining enzymes, which will reveal important biochemical targets (Korstanje and Paigen, 2002).

Natural genetic variation controlling various traits has intensively been studied using recombinant inbred lines (RILs) in plants, especially in the model species Arabidopsis thaliana (Alonso-Blanco et al., 2009). Several genes regulating glucosinolate contents have been elucidated through linkage mapping in Arabidopsis (Pfalz et al., 2009; Zhang et al., 2006). Except two studies about loci related to levels of abscisic acid (ABA) in maize and salicylic acid (SA) in Arabidopsis (Dobon et al., 2011; Tuberosa et al., 1998), to our knowledge, there are at present no QTL-based data yet on genetic elements determining hormone contents in Arabidopsis or other plant species, despite the crucial roles of hormones in plant growth and development.

In this study, we investigated if, and to what extent, a quantitative genetic approach based on hormone levels in roots, may reveal genes involved in regulatory or metabolic pathways. We chose a RIL population, derived from the parental lines Landsberg erecta (Ler-0) and Cape Verde Island (Cvi0 ), since these lines showed divergent traits for root hormone contents in our previous study (Lee et al., 2017). Several QTLs for levels of cytokinins (CKs) and jasmonic acid (JA) were confirmed using 
near-isogenic lines (NILs). Further, by using a loss-of-function mutant of a CK $N$-glucosyltransferase gene situated at a QTL region of CK metabolites, its functional role was evaluated.

The present study shows that genetic elements controlling hormone levels in plants can be unravelled through quantitative genetic analysis, providing a powerful method to understand hormone metabolism.

\section{Materials and methods}

\section{Plant materials and phenotyping}

Ler $\times$ Cvi RILs, developed and genotyped by Alonso-Blanco et al. (Alonso-Blanco et al., 1998b), were chosen based on our previous study on the natural variation of hormone levels in roots of Arabidopsis accessions (Lee et al., 2017). Seeds of 149 lines were placed on wet filter paper in a Petri-dish at $4{ }^{\circ} \mathrm{C}$ for 4 days in darkness and after that sown on the top of $0.5 \mathrm{~mL}$ cylindrical plastic tube, from which the bottom had been cut off, and that was filled with $0.5 \%$ agar in half strength of Hoagland's nutrient solution ( $\mathrm{pH}$ 5.5). Tubes with seedlings of each line were grown in hydroponic containers (70 plants per 10 litres, renewing the nutrient solution once a week). Plants were grown at $21^{\circ} \mathrm{C}$ during the light period $(10 \mathrm{~h})$ and at $18^{\circ} \mathrm{C}$ during the dark period $(14 \mathrm{~h})$. Light intensity and humidity were fixed at $125 \mu \mathrm{Mol} \mathrm{m} \mathrm{s}^{-1}$ and $70 \%$ respectively.

After 5 weeks of culture, roots were harvested between the 5th and 8th hour within the 10 hours daytime period. For hormone analysis of RILs, NILs and knockout (KO) plants, six to seven roots of each line were pooled for one biological replicate. Pooled roots were immediately ground in liquid nitrogen and freeze-dried for 24 hours. For hormone analysis in leaves (Columbia-0 and KO plants), two largest rosette leaves in each plant were chosen and leaves of four plants were pooled for one biological replicate. On the day of harvest, the developmental stage (vegetative or flowering) and root phenotypic traits of each line were recorded. If any of the replicates within a line showed visible bolting, the line was scored as 'flowering'. Before phenotyping of root fresh weight, the drops of liquid on roots were removed with paper tissue.

\section{Hormone extraction and purification}

For each RIL, one biological replicate was used to extract endogenous hormones and further analysed for hormone quantification. For parental lines, NILs and KO plants, 4 to 5 biological replicates were used. Powder of lyophilized roots $(2.5 \mathrm{mg})$ was extracted and purified with the same methods used in our previous study (Lee et al., 2017). 


\section{Quantitative analysis of plant hormones}

For IAA, ABA, CKs, JA and SA, all of ultra-pressure liquid chromatography (UPLC)-tandem mass spectrometer methods were the same as those of our previous study (Lee et al., 2017). In order to achieve better chromatographic separation of isomers of CK glucoside (e.g., tZ7G, tZ9G and tZOG) in NILs and KO plants, ammonium formate was used as described by Novak et al. (2008). Ten microliter of sample was injected onto an Acquity UPLC HSS column (50 x $2.1 \mathrm{~mm}, 1.8 \mu \mathrm{m}$; Waters) and eluted by binary mobile phases, A (15mM ammonium formate, $\mathrm{pH} 4.0)$ and $\mathrm{B}(100 \%$ methanol), with a constant flow rate $\left(0.25 \mathrm{ml} \mathrm{min}{ }^{-1}\right)$ at $40^{\circ} \mathrm{C}$ for $14 \mathrm{~min}$. The linear gradient elution was performed as follows: $0 \sim 0.01 \mathrm{~min}, 10 \%$ eluent $\mathrm{B}$; $0.01 \sim 8.0 \mathrm{~min}, 10$ to $50 \%$ eluent $\mathrm{B}$; 8.0 8.5 min, 50 to $100 \%$ eluent $\mathrm{B}$; $8.5 \sim 9.5 \mathrm{~min}, 100 \%$ eluent B; 9.5 10.5 min, 100 to $10 \%$ eluent $\mathrm{B}$. At the end of gradient, the column was equilibrated to initial conditions for $3.5 \mathrm{~min}$. The effluent was introduced in electrospray ion (ESI) source of mass spectrometer with operating parameters: capillary voltage, 3 $\mathrm{kV}$; cone voltage, $22 \mathrm{~V}$; source and desolvation temperature, $150^{\circ} \mathrm{C}$ and $650^{\circ} \mathrm{C}$; cone and desolvation gas flow, 50 and $1000 \mathrm{~L} \mathrm{hour}^{-1}$; MS mode collision energy, $2 \mathrm{~V}$; MS/MS mode collision energy, $10 \mathrm{~V}$. Two selective transitions were used to perform multiple reaction monitoring (MRM) detections (S1 Table). All data were processed by TargetLynx in MassLynx ${ }^{\text {TM }}$ Software (Version 4.1, Waters, USA). The quantification of each targeted analyte was based on a linear calibration curve that covered the range of concentrations of compounds in samples, and corrected by the recovery rates of the isotopelabelled internal standards.

\section{QTL analysis}

To map QTLs using the RIL population, a set of 99 markers spaced over the Arabidopsis genetic map was selected from the previous published RIL Ler/Cvi map (Alonso-Blanco et al., 1998b). These markers spanned $482 \mathrm{cM}$, with an average distance between consecutive markers of $5 \mathrm{cM}$ and the largest genetic distance being $12 \mathrm{cM}$. QTL analysis was performed using the computer program MapQTL version 6.0 (Ooijen, 2004) as described by Bentsink et al. (Bentsink et al., 2003). Both interval mapping and multiple QTL model (MQM) methods were used to locate QTLs linked to the molecular markers as described in the reference manual. The estimated additive effect and the percentage of variance explained by each QTL affecting a trait, were obtained with MapQTL in the final MQM model. For this, different cofactor markers were tested around the putative QTL positions (Ooijen, 2004), selecting as final cofactors the closest marker to each QTL. A logarithm of odds (LOD) threshold of 2.5 was applied to declare a significant QTL, which corresponds to a general genome-wide significance of $\mathrm{P}<0.05$, as determined by permutation tests ( 1,000 repetitions). QTL regions for $95 \%$ confidence were determined by 2 -LOD support interval that constructs two positions, left and right of the point estimate of the QTL, which have a LOD score of two less than the maximum. 
For the purpose of revealing developmental stage-specific QTLs, all RILs were sorted into two groups according to the transition of plants for flowering at day 35, i.e., the day of sampling (the ratio between vegetative and flowering line was 48.9 : 51.1). For each trait in the two groups, QTL regions were independently determined by MQM analysis with automated suggested cofactors.

\section{QTL confirmation analysis}

A selected set of NILs carrying small Cvi introgressions in Ler background, which was developed by Keurentjes et al. (2007a), was tested to confirm some chosen QTLs in the Ler $\times$ Cvi RIL population. Significant differences of tested traits were compared between Ler and NILs through analysis of variance (ANOVA, $\mathrm{p}<0.05)$.

\section{PCR to confirm homozygous KO plants}

Candidate T-DNA (KO) plants were chosen from TAIR (www.arabidopsis.org) website and obtained from the European Arabidopsis Stock Centre (NASC, UK). The PCR was performed to screen TDNA insertion and its homozygosity for the gene of interest. Forward and reverse primers were designed on the website, T-DNA Primer Design (http://signal.salk.edu/tdnaprimers.2): for SAIL 801B03 and SAIL 1151A08, forward 5'-TCGAAAAACGTCAACAAAACC-3', reverse 5'AGAGTCCTCTGCTTCCGATTC-3'; SALK 102337, forward 5'GCAGATCATAGGAACCCCTTC3', reverse 5'- TCCGAACCAAGGGATATCTTC-3'. Reaction mixtures were prepared with reagents: $1 \mu \mathrm{l}$, d'NTPs $(5 \mathrm{mM}) ; 0.4 \mu 1$, forward and reverse primer (10 pM); $0.15 \mu 1$, Firepol (1U); $7.15 \mu 1$, water; $1.2 \mu 1$, PCR buffer; $1.5 \mu 1, \operatorname{MgCl}(2.5 \mathrm{mM}) ; 1.0 \mu 1$, DNA. The following conditions were performed to amplify DNA fragments: denaturation at $95{ }^{\circ} \mathrm{C}$ for $5 \mathrm{~min}$ followed by $30 \mathrm{~s}$ at $95{ }^{\circ} \mathrm{C}$, annealing at $55^{\circ} \mathrm{C}$ for $30 \mathrm{~s}$ and extension at $55^{\circ} \mathrm{C}$ for $2 \mathrm{~min}$, which was cycled 30 times and ended with final amplification at $72{ }^{\circ} \mathrm{C}$ for $10 \mathrm{~min}$.

\section{Results}

\section{Variation of hormone levels in roots of Ler $\times$ Cvi RIL population}

To identify the genetic loci affecting endogenous hormone levels in Arabidopsis roots, hormone levels were determined in 35-day-old-roots of a Ler $\times$ Cvi RIL population. For most hormones and their metabolites, transgressive segregation was found within the population (Fig 1), the levels ranging 10 - to 30 -fold between extremes. It indicates that both parental lines carry allelic variants that increase or decrease values of traits. For ABA, only a limited range of concentration variation was observed, viz., three-fold between extremes. In most cases, normal distributions were found, although the distribution ranges were skewed for several compounds, e.g. cis-zeatin riboside (cZR) and JA. Weight phenotypic traits showed transgressive segregations below the parental values. 

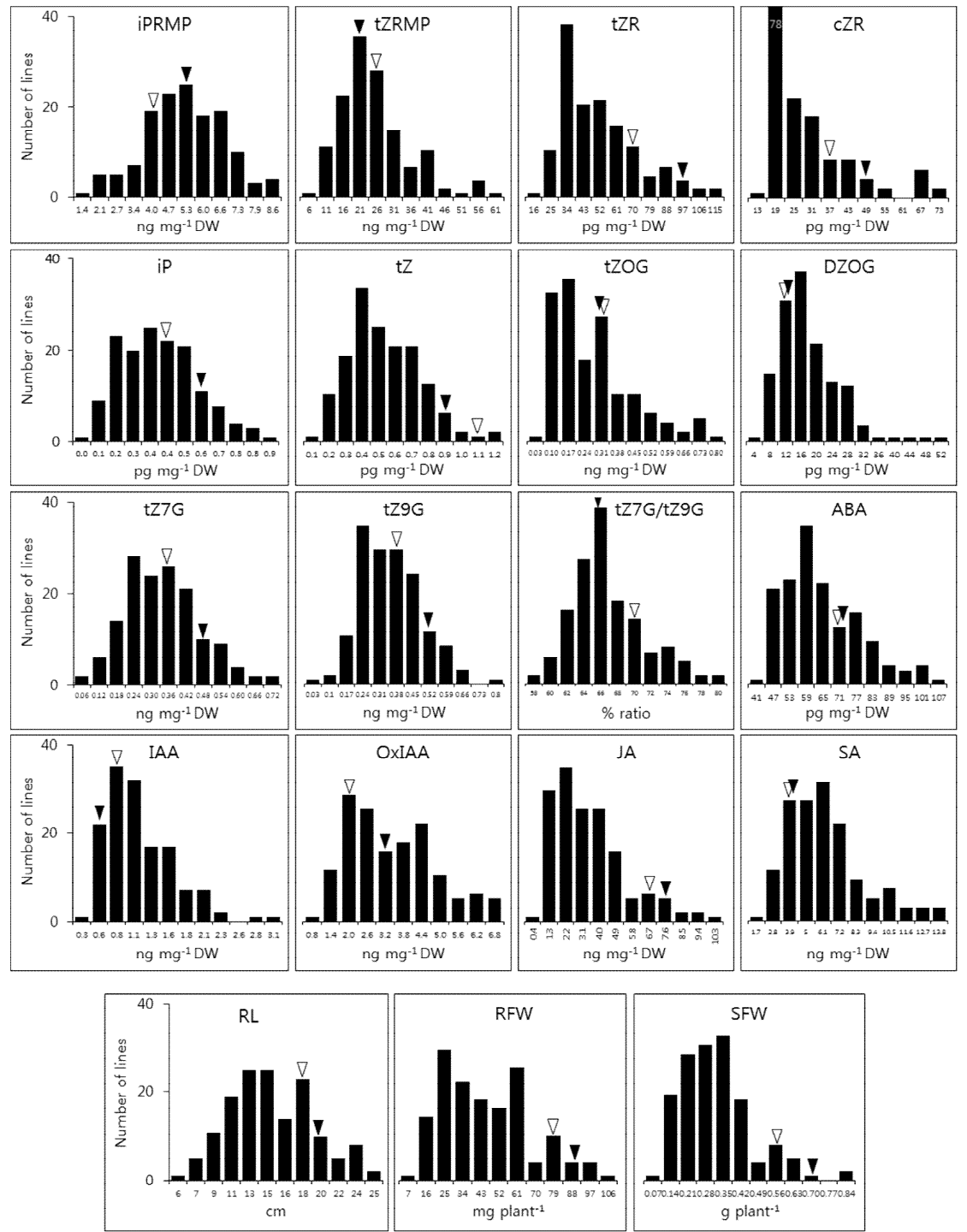

Figure 1. Frequency distributions of root hormone levels and phenotypic traits in the Ler $\times$ Cvi RIL population. Arrows indicate levels of hormones in parental lines: Ler, black; Cvi, white. Abbreviations for hormone compounds and phenotypic traits are: isopentenyl riboside monophosphate (iPRMP), trans-zeatin riboside monophosphate (tZRMP), ), trans-zeatin riboside (tZR), cis-zeatin riboside (cZR), isopentenyl ladenine (iP), trans-zeatin (tZ), trans-zeatin-O-glucoside (tZOG), dihyro-zeatin-O-glucoside (DZOG), trans-zeatin-7,9glucoside (tZ7,9G), abscisic acid (ABA), indole-3-acetic acid (IAA), 2-oxindole-3-acetic acid (OxIAA), jasmonic acid (JA), salicylic acid (SA), root length (RL), root fresh weight (RFW) and shoot fresh weight (SFW). 
In several cases, significant correlations were found between levels of hormone compounds (Table 1). Among CK metabolites, $t Z$ positively correlated with its two precursor metabolites, tZRMP and tZR. The two CK $N$-glucosides (tZ7G and tZ9G) showed highly significant positive correlations between each other, and both were positively correlated with tZOG. A significantly positive correlation was found between the two CK ribosides (tZR and cZR), and both of them showed similar pattern of correlations to other hormones. 2-oxindole-3-acetic acid (OxIAA) that is known as an irreversible catabolite of IAA (Tanaka et al., 2014; Zhao et al., 2013) negatively correlated with IAA. Low correlation values were observed between ABA and other hormones. Also, some correlations between phenotypic traits and hormones were found. Root fresh weight (RFW) and root length (RL) showed significantly negative correlations with levels of JA, whereas levels of CK glucosides positively correlated with RFW and shoot fresh weight (SFW).

Table 1. Correlations between hormone levels and phenotypic traits in roots of 35-day-old plants of the Ler $\times$ Cvi RIL population. Star marker $\left(^{*}\right)$ indicates the significant correlation $(\mathrm{p}<0.01)$. Abbreviations for hormone compounds and phenotypic traits are: isopentenyl riboside monophosphate (iPRMP), isopentenyladenine (iP), trans-zeatin riboside monophosphate (tZRMP), trans-zeatin riboside (tZR), trans-zeatin (tZ), trans-zeatin-O-glucoside (tZOG), trans-zeatin-7,9-glucoside (tZ7,9G) dihyro-zeatin-O-glucoside (DZOG), cis-zeatin riboside (cZR), indole-3-acetic acid (IAA), 2-oxindole-3-acetic acid (OxIAA), abscisic acid (ABA), jasmonic acid (JA), salicylic acid (SA), root length (RL), root fresh weight (RFW) and shoot fresh weight (SFW).

\begin{tabular}{|c|c|c|c|c|c|c|c|c|c|c|c|c|c|c|c|}
\hline Variables & iPRMP & iP & tZRMP & tZR & tz & tZ7G & tZOG & tZ9G & DZOG & cZR & IAA & OxIAA & $\mathrm{ABA}$ & $J A$ & SA \\
\hline iP & 0.18 & & & & & & & & & & & & & & \\
\hline tZRMP & 0.21 & 0.02 & & & & & & & & & & & & & \\
\hline tZR & $-0.23^{*}$ & -0.12 & $0.31^{*}$ & & & & & & & & & & & & \\
\hline tz & 0.10 & 0.03 & $0.22 *$ & $0.42^{*}$ & & & & & & & & & & & \\
\hline tZ7G & 0.06 & 0.13 & -0.11 & -0.06 & 0.16 & & & & & & & & & & \\
\hline tZOG & -0.01 & 0.19 & -0.13 & -0.12 & 0.10 & $0.22^{*}$ & & & & & & & & & \\
\hline tZ9G & 0.19 & 0.21 & -0.14 & -0.20 & 0.12 & $0.83^{*}$ & $0.22^{*}$ & & & & & & & & \\
\hline DZOG & 0.10 & 0.17 & -0.03 & $-0.22 *$ & 0.07 & $0.38^{*}$ & 0.12 & $0.35^{*}$ & & & & & & & \\
\hline cZR & $-0.40 *$ & $-0.24 *$ & -0.04 & $0.57^{*}$ & $0.21 *$ & 0.09 & -0.10 & -0.12 & -0.18 & & & & & & \\
\hline IAA & 0.20 & 0.11 & 0.14 & 0.05 & 0.03 & $-0.24 *$ & -0.05 & -0.09 & -0.01 & -0.08 & & & & & \\
\hline OxIAA & -0.19 & -0.09 & 0.11 & $0.22^{*}$ & 0.16 & 0.16 & -0.02 & 0.00 & -0.06 & $0.29^{*}$ & $-0.33^{*}$ & & & & \\
\hline$A B A$ & -0.02 & 0.10 & 0.05 & -0.02 & -0.03 & -0.06 & -0.09 & -0.06 & 0.01 & 0.10 & 0.19 & -0.05 & & & \\
\hline$J A$ & -0.13 & $-0.23^{*}$ & -0.03 & $0.32^{*}$ & $0.23 *$ & 0.13 & -0.07 & -0.12 & -0.18 & $0.51^{*}$ & -0.18 & $0.38^{*}$ & -0.04 & & \\
\hline SA & 0.12 & 0.01 & -0.01 & 0.00 & -0.09 & $-0.27^{*}$ & 0.04 & -0.16 & 0.00 & -0.08 & $0.33^{*}$ & $-0.23 *$ & 0.11 & -0.04 & \\
\hline $\mathrm{RL}$ & 0.21 & 0.04 & -0.07 & -0.11 & 0.00 & -0.05 & $0.21^{*}$ & 0.09 & -0.08 & -0.18 & 0.15 & $-0.23 *$ & -0.08 & $-0.21^{*}$ & 0.02 \\
\hline RFW & 0.19 & 0.20 & -0.06 & $-0.30^{*}$ & -0.05 & 0.14 & $0.30^{*}$ & $0.32 *$ & 0.04 & $-0.27^{*}$ & -0.03 & 0.05 & -0.05 & $-0.28^{*}$ & -0.20 \\
\hline SFW & 0.03 & 0.08 & -0.07 & -0.05 & 0.07 & $0.35^{*}$ & $0.30 *$ & $0.39 *$ & 0.00 & -0.06 & -0.15 & $0.31 *$ & -0.13 & 0.01 & $-0.29 *$ \\
\hline
\end{tabular}




\section{QTLs for CKs, SA, JA and root phenotypic traits}

Among five classes of hormone, significant QTLs for CK, JA, SA and several phenotypic traits were observed (Fig 2, S2 Table). The explained variances of these loci for the various traits ranged from $5 \%$ (tZOG on the chromosome 4) up to $25 \%$ (tZ9G on the chromosome 5). Twelve QTL regions related to CK metabolites and only one QTL for JA and SA were observed. Within CK metabolites, a few QTLs co-localized, but the directions of additive effects of these were not the same. No significant QTLs were found for IAA, OxIAA and ABA.

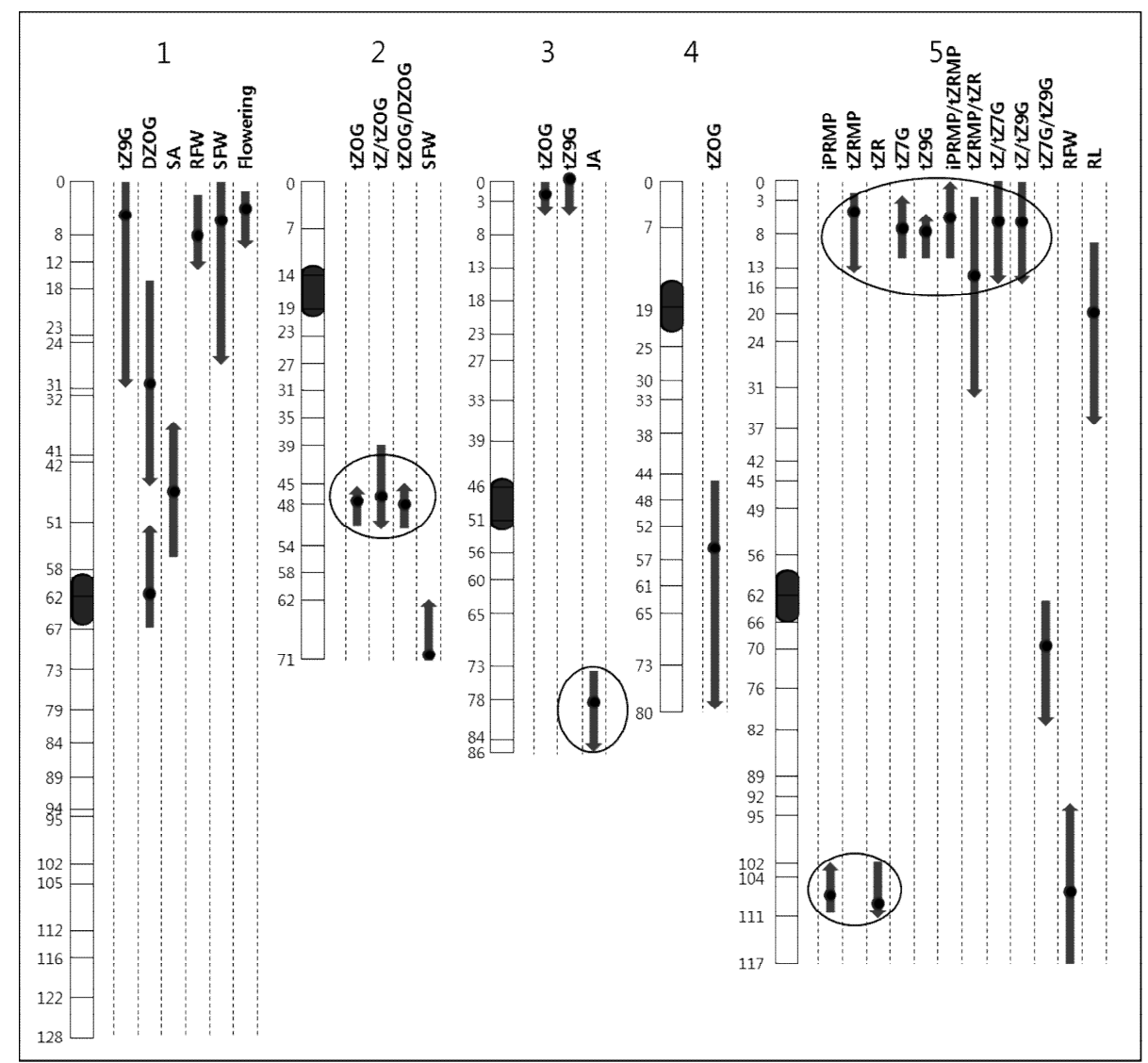

Figure 2. Genetic locations of hormonal and root phenotypic QTLs. Numbered bars represent chromosomes; the numbers along the bars are genetic map position in centi-Morgans. Directions of arrows indicate additive (allelic) effects: upward, Ler alleles increase trait values; downward, Cvi alleles increase trait values. Lengths of arrows indicate 2-LOD intervals (95\% confidence). Dots in arrows indicate the highest LOD position for each QTL. Circles indicate four QTL regions that were tested with related NILs for QTL confirmation analysis. 
QTLs for levels of tZ9G, DZOG and SA were detected at the upper arm of chromosome 1, but with different positions. The QTLs for tZ9G co-localised with those for RFW, SFW and flowering time, and they all showed the same allelic effect. This region coincides with the earlier reported QTLs for flowering time and root growth in the same RIL population (El-Din El-Assal et al., 2001; Keurentjes et al., 2007a; Sergeeva et al., 2006). Interestingly, a QTL for tZ9G was found, but no significant QTL for its isomer (tZ7G) was detected at the same region.

Biosynthetic pathways in plant metabolism are composed of a series of precursor-to-product pathway, in which rate-controlling steps (usually enzymatic reactions) are involved (Harpaz-Saad et al., 2007; Hasunuma et al., 2010). Several cases of such precursor-to-product relation existed within CK metabolites measured in this study. Since most of the conversions are catalysed by enzymes, which are encoded by genes, we expected that QTLs for ratios of metabolic compounds should be also detectable. Indeed, within CK metabolites, six metabolite ratio QTLs were found. Most of these QTLs co-localized with those of single CK compounds except one for $\mathrm{tZ7G/tZ9G} \mathrm{ratio,} \mathrm{which} \mathrm{was}$ newly detected at the lower arm of chromosome 5 .

\section{Development stage-dependent QTLs for hormone levels}

At the time the samples for hormone analyses were taken ( 35 day), 51\% ( 76 out of total 149 ) of the lines were visibly bolting. Therefore, we wondered if QTL analysis could be done for vegetative and flowering lines separately, and to what extent the results would be different from the analysis based on the whole population. Using the same RIL population, Keurentjes et al. (2007b) demonstrated that values of LOD and the number of detected QTLs decreased with decreasing population size and that the degree of the changes differed from trait to trait. In our case, most QTLs detected in the whole population were detectable in the sub-populations, but some of them were no longer significant. Among 23 significant QTLs for hormones and root traits found in the whole population, 13 QTLs appeared to be below the significance threshold (2.5 LOD score) due to lower power in the smaller subsets. Interestingly, some new QTLs were detected in the subsets that had not been detected in the full set of lines.

Fifteen of the QTLs from the sub-sets could be categorized into three classes: vegetative-specific (8 QTLs), flowering-specific (5 QTLs) and non-development-specific (2 QTLs) (Fig 3). Among the 8 QTLs specific for the vegetative stage, two QTLs for SA and RL were newly detected in vegetative lines and had not been found in the whole population ( ${ }^{\S}$ superscript marked in Fig 3, S2 Table). In some cases, two developmentally distinct QTLs for the same trait were observed: two QLTs for RFW, one for the vegetative stage on the top of chromosome 1, the other specific for the flowering stage on chromosome 5; two QTLs for tZ9G, one for vegetative stage on the top of chromosome 3 and the other for flowering stage on chromosome 5. For the level of tZOG, two QTLs were found, one on the 
top of chromosome 3 was flowering-specific, and the other on chromosome 2 was non-developmentspecific. Among four QTLs for metabolite-ratio traits, developmental specificities of three QTLs were the same as those for either precursors or products, e.g., flowering-specific QTLs for the level of tZRMP and tZRMP/tZR ratio on chromosome 5. This implies that the same genetic loci may underlie both QTLs, being functional during the same developmental stage.

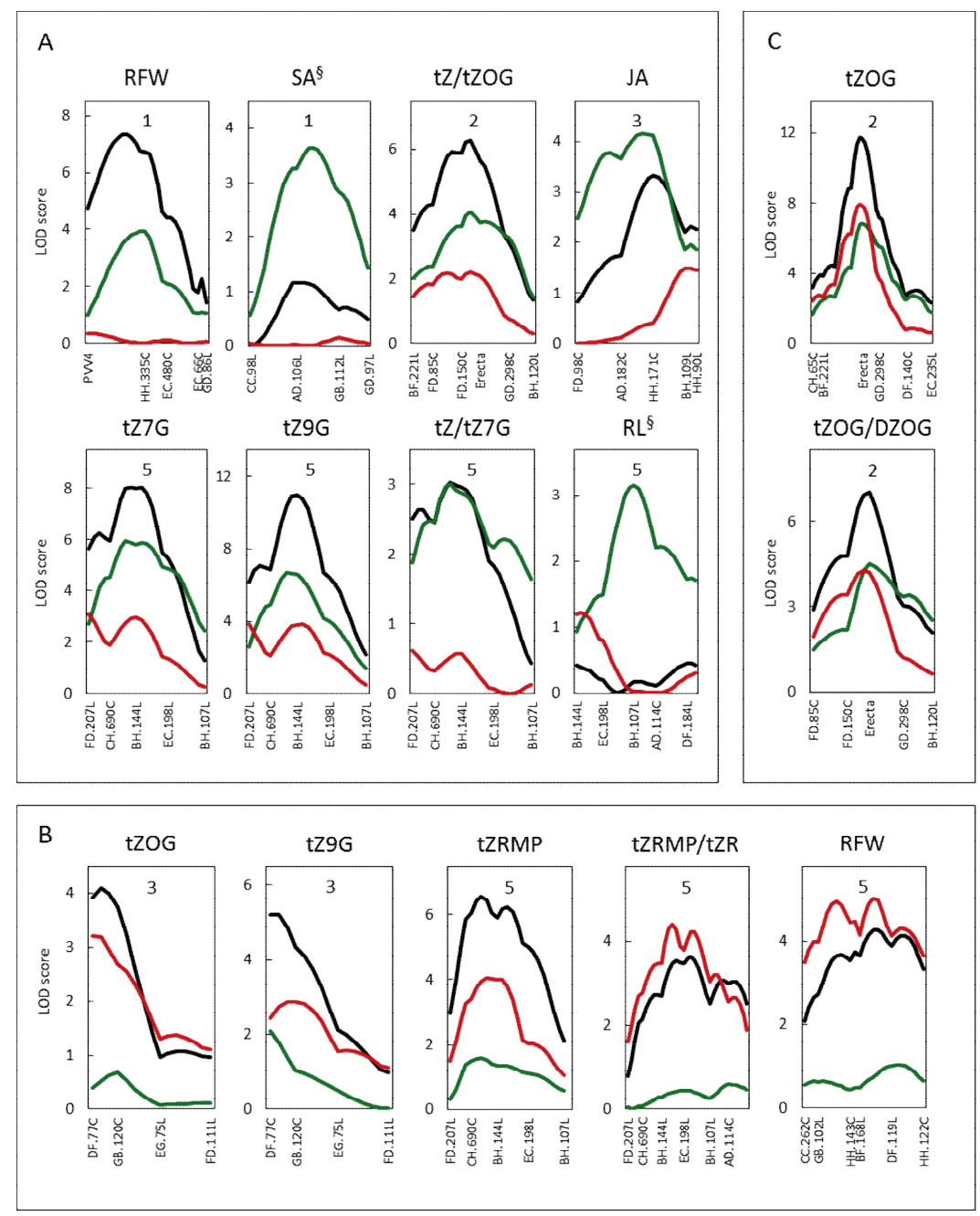

Figure 3. Development-specific QTLs based on vegetative- and flowering lines. (A) vegetative-specific QTLs. (B) flowering-specific QTLs. (C) non-development-specific QTLs. X-axes present genetic markers. Black lines are QTL-profiles when all lines (149) were included, green lines from only vegetative plants, and red lines from only flowering plants. Numbers in the boxes refer to chromosome number. Only the parts of chromosomes containing significant QTLs are depicted. 


\section{Confirmation analyses of hormone QTLs}

Four QTL regions, affecting levels of CK and JA, were further investigated using available NILs, with small Cvi introgressions into Ler background (Keurentjes et al., 2007a).

a. QTL for CK metabolites at chromosome 5: Maps of two NILs (5-1 and 5-2) and QTL regions for three CK compounds and their metabolite-ratios at the top of chromosome 5 are described in Fig 4A. For tZ7G and tZ9G, significant lower levels of the compounds were observed in both NILs, and NIL5-1 showed significantly lower levels of compounds than NIL5-2 (Fig 4B). It implies that the QTL region may include two different loci, being divided around BH.144L, one in the upper position having a stronger effect than the other in the lower position. Both NILs showed higher levels of tZRMP compared to Ler, which is consistent with the presence of a locus affecting tZRMP level, although the differences were not significant.

b. QTL for tZOG at chromosome 2: A large-effect QTL (11.7 LOD score) for the level of tZOG on chromosome 2 was confirmed using three NILs (S1A Fig). Significant lower levels of tZOG were observed in both NIL2-17 and NIL2-18, but not in NIL2-8 (Fig 5A). This might indicate that a responsible gene is located slightly below the above-described major QTL position, or that more than one locus is involved. In the overlapping introgression region of NIL2-17 and NIL2-18 several genes related to CK biosynthetic pathways are located (LOG2, LOG3, UGT73C1 and UGT73C5) (Hou et al., 2004; Kuroha et al., 2009).

c. QTL for iPRMP and tZR at chromosome 5: A locus for levels of iPRMP and tZR on the bottom of chromosome 5 was evaluated using NIL5-15. The NIL showed significantly lower level of iPRMP than Ler, but the level of tZR was similar to that of Ler (Fig 5B, S1B Fig). These results suggest that a locus for the lower level of iPRMP is indeed located in the Cvi introgression region. While, a locus for the higher level of tZR may be located below CH.331L-Col because the introgression region for Cvi alleles in NIL5-15 does not fully cover the QTL region between CH.331L-Col and EG.205L. However, since these were only minor QTLs (2.5 3.0 LOD scores), the statistical power in the analyses might have been too low to detect allelic effects in the NILs. A known CK oxidase/dehydrogenase (CKX3) locates at the top of both QTL and the Cvi introgression in NIL5-15 (Bartrina et al., 2011). 

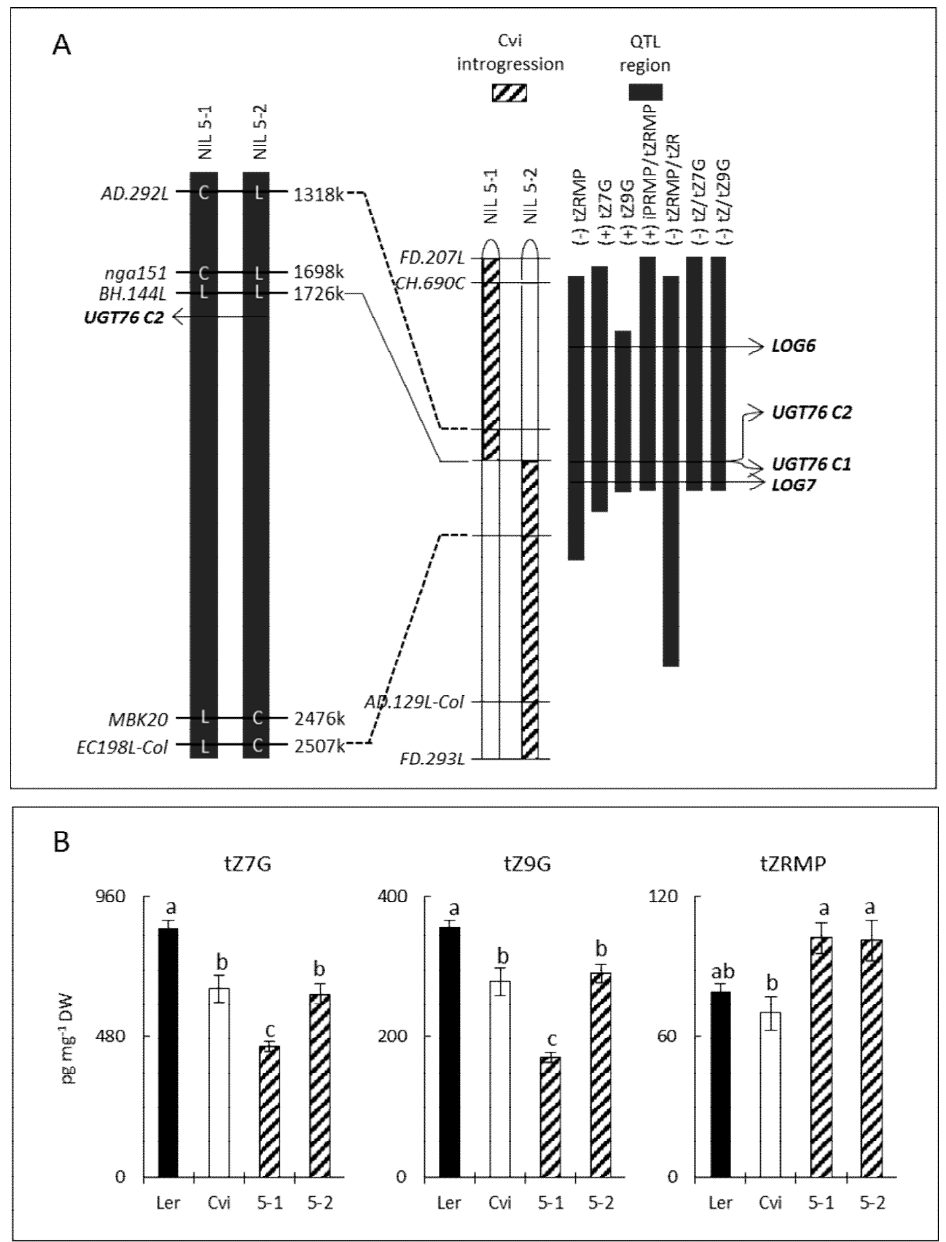

Figure 4. Confirmation of QTLs for levels of tZ7G, tZ9G and tZRMP. (A), genotype of NIL5-1 and NIL5-2. $\mathrm{L}$ (Ler-0) and C (Cvi-0) in the black columns on the left indicate allelic identities of parental lines at the markers found in the two NILs. In the middle bars, diagonally-striped areas represent Cvi introgression regions in the NILs. On the right, black columns indicate QTL regions in 2-LOD confidence interval for traits of tZ7G, tZ9G, tZRMP and CK metabolite-ratios. Plus and minus in parentheses in front of trait names indicate additive effects. Horizontal arrows represent positions of known genes related to CK biosynthetic pathways in Arabidopsis: two Lonely Guy (LOG) genes and two CK $N$-glucosyltransferases (CK-UGT). (B), compound levels in tested lines, (Ler-0, Cvi-0, NIL5-1 and NIL5-2). Vertical lines on the bars present standard errors and letters on the top of each bar indicate significant differences between lines with a confidence interval of $95 \%$ (ANOVA, Duncan). 

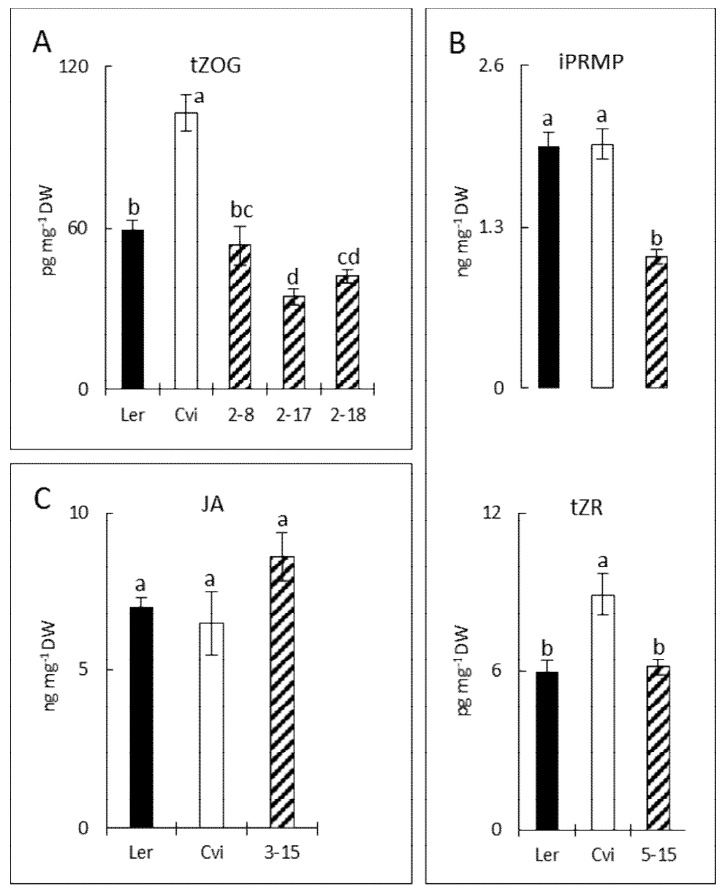

Figure 5. Confirmation of QTLs for tZOG, iPRMP, tZR and JA. (A), tZOG levels found in parental lines and three NILs, with Cvi-0 introgression in Ler-0 background (2-8, 2-17 and 2-18). For details, see maps of NILs in the supporting information (S1A Fig). (B), levels of iPRMP and tZR in NIL5-15 (S1B Fig). (C), JA levels in NIL3-15 (S1C Fig). Vertical bars present standard errors and letters on the top of each bar indicate significant differences between lines with a confidence interval of $95 \%$ (ANOVA, Duncan).

d. QTL for JA at chromosome 3: The QTL for the level of JA at the bottom of chromosome 3 was evaluated with NIL3-15 (S1C Fig). The level of JA in NIL3-15 was higher than that in Ler, although there was no significant difference (Fig 5C). Since the region of Cvi introgression in NIL3-15 fully covers the significant interval of the QTL, a locus for the higher level of JA in Cvi can be located in between AD.182C and BH.109L-Col.

\section{Effects of loss-of-function of $U G T 76 C 2$ on CK metabolites and root phenotypic traits}

To further elucidate genes affecting levels of CKs (tZRMP, tZ7G and tZ9G), we zoomed in on QTL regions at the top of chromosome 5. At this region (Fig 4A), containing QTLs for tZRMP, tZ7G, tZ9G and some of CK metabolite-ratios, several known CK genes are situated: two LOGs (LOG6 and LOG7) and two UGTs (UGT76C2 and UGT76C1) (Kuroha et al., 2009). Lonely Guy (LOG) converts cytokinin nucleoside 5'-monophosphates (e.g., iPRMP and tZRMP) to free-bases (e.g., iP and tZ) 
(Kurakawa et al., 2007). Biologically active free bases are deactivated by $N$-glucosyltransferase, resulting in e.g., tZ7G (Hou et al., 2004; Kuroha et al., 2009).

All these biosynthetic genes are supposed to be directly responsible for determining concentrations of tZRMP, tZ7G and tZ9G detected in the present QTL analysis. Thus, we chose LOG7 gene (At5g06300) and two zeatin $N$-glucosyltransferase genes (At5g05860 for UGT76C2 and At5g05870 for UGT76C1) that are known to be clearly expressed in roots of young seedling stages, as genetic components for further $\mathrm{KO}$ analyses (Winter et al., 2007).

Among the tested $\mathrm{KO}$ lines for these genes, we obtained a homozygous T-DNA insertion line (SAIL 801B03) of UGT76C2 gene (Fig 6A, S2 Fig), which is known to be higher expressed in roots during the vegetative stage, as compared to UGT76C1 (Smehilova et al., 2016). For genes of LOG7 and UGT76C1, no homozygous T-DNA insertion line was obtained from the given KO lines.

Fig 6B illustrates part of the CK biosynthetic pathways and the changes of metabolic contents between the wild type and the KO plant. The KO plant of UGT76C2 showed dramatic decreases of concentrations of tZ7G and tZ9G in roots. Levels of other CK $N$-glucosides (iP9G, DZ7G and DZ9G) from different side chains also decreased, which may be direct consequences of the loss-of-function of the gene. However, levels of CK $O$-glucosides (iPOG and tZOG) in both roots and leaves were hardly changed, showing $\mathrm{N}$-glucosylation specificity of UGT76C2. The knocking-out of the gene also affected a wide range of levels of other CK metabolites. In roots, levels of the various metabolites (iPR, iP, tZRMP, tZR, tZ7G, tZ9G, DZ7G, DZ9G, cZR and cZG) decreased, whereas in leaves also opposite effects were found (iP and cZ). For this KO plant, significant morphological changes in roots were also observed. RL and RFW decreased by more than $60 \%$ in the KO plant compared to the wild type (Fig 6C), while only $9 \%$ reduction of SFW was observed.

\section{Discussion}

\section{Hormone quantities and their correlations}

A wide range of concentrations of hormones and their metabolites were found in the RILs. Except for $\mathrm{ABA}$ and cZR, concentration differences were several ten-fold between extremes in the segregating population, which was in strong contrast to the very limited variations of hormone levels found in 23day-old roots of 13 Arabidopsis natural accessions in our previous study (e.g., iP and tZ, < 1.5 fold differences) (Lee et al., 2017). Transgressive segregation has been found in many studies using biparent populations (Bentsink et al., 2010; Keurentjes et al., 2007a; Vreugdenhil et al., 2004). 


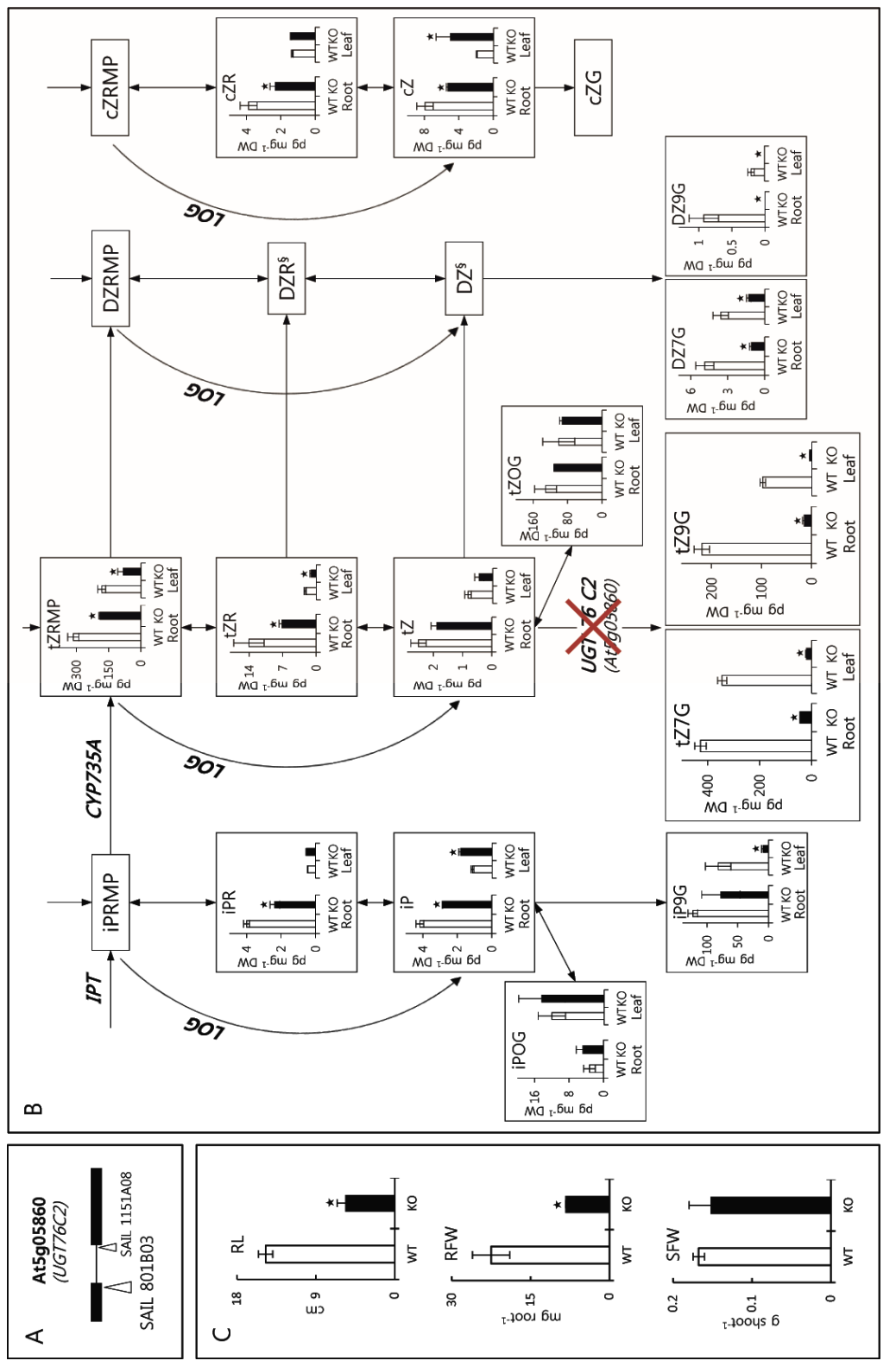

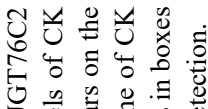

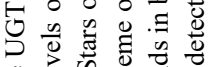

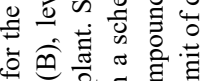
น 品怘导导 일

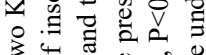
उ 400

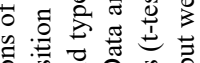

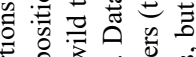

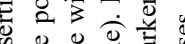

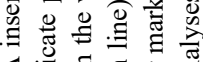
$\varangle$ 淃 1 识 क

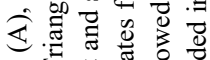
부

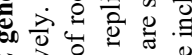

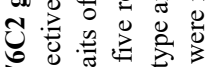
5 क

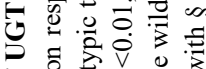

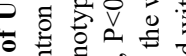

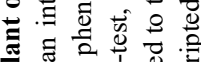

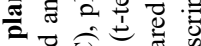
○芯包芯芯

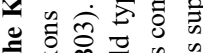

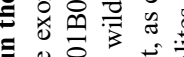

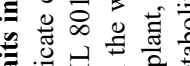

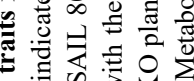

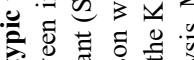

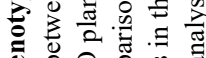
을

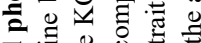

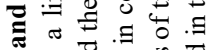

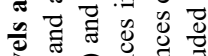
¿

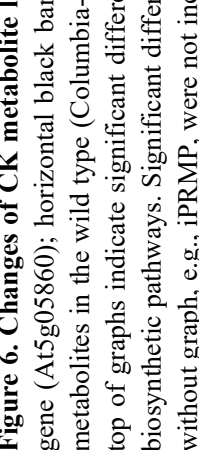


The most likely explanation for this phenomenon is the presence of both positive and negative alleles in each of the parental lines. In the RILs new allelic combinations will occur, leading to more extreme phenotypes. The limited natural variation, as observed in the accessions, points to natural selection leading to less extreme phenotypes.

Positive correlations between levels of $\mathrm{tZ} \mathrm{N}$-glucosides and RFW are similar to our previous results on natural accessions $\left(r^{2}=0.48 \sim 0.57\right)$ (Lee et al., 2017). These are in agreement with the findings of Novak et al. (Kollmer et al., 2014) showing that the reduction of CK $\mathrm{N}$-glucoside levels caused by $\mathrm{CK}$ oxidase/dehydrogenase (CKX7) overexpression resulted in the reduction of primary root elongation and lateral root growth. In the present study, similar morphological changes in root development were found in the $\mathrm{KO}$ plant of $\mathrm{CK} N$-glucosyltransferase, proving a significant role of $\mathrm{CK} N$-glucoside in root growth and development (Fig 6C). The underlying mechanism on how $\mathrm{CK}$ glucosides play a role in promoting root growth is unknown, but it has been used for exogenous application as a sugar-conjugate plant growth regulator (Nonomura et al., 2011). The significant role of CK $\mathrm{N}$-glucoside in plant productivity suggests that the regulation of CK glucoside contents can be a biochemical target in breeding programs, particularly for root systems.

Significant correlations between hormone compounds may point to common molecular mechanisms in their metabolism. It is noteworthy that a high correlation $\left(\mathrm{r}^{2}=0.83\right)$ between $\mathrm{tZ7G}$ and tZ9G was observed. The average concentration ratio (peak area comparison in chromatograms) observed in the whole RILs was $0.97: 1$ (tZ7G : tZ9G). The transgressive segregation of this trait (Fig 1) suggests that the ratio may be a consequence of polygenic inheritance. Hou et al. (2004) suggested that the abundance of $\mathrm{tZ7G}$ compared to $\mathrm{tZ9G}$ may result from the specificity of $\mathrm{CK} \mathrm{N}$ glucosyltransferases (thus a biological reaction), but the amount of tZ9G may also be the result of tautomerism between two constitutional isomers in aqueous solution (non-biological reaction). But Leon et al. (Leon, 1998) estimated that $N 9$-glucosylation of $\mathrm{tZ}$ is rather stable in relation with entropic contribution $\left(\Delta \mathrm{G}^{\circ}\right)$, concluding that approximately equal or lower amounts of $N 7$-isomer should be present at room temperature. In the present study, the existence of at least two independent QTLs for tZ9G level provides empirical evidence that the quantitative genetic component is involved in determining the trait (Fig 2), implying that an unknown enzymatic pathway (isomerization) might be involved in the conversion of two CK $N$-glucoside isomers.

\section{QTLs for hormone metabolites and their ratio traits}

The total number of significant QTLs for 15 metabolites in five groups of hormones was 14 . The average number of QTL per hormone metabolite was strikingly lower compared to those of diverse growth-related traits, mineral contents, or seed traits as reported in previous studies using the same RIL population (Alonso-Blanco et al., 1999; Alonso-Blanco et al., 1998a; Bentsink et al., 2003; 
Sergeeva et al., 2004; Vreugdenhil et al., 2004). Except for the two loci for tZOG, tZ7G and tZ9G, most QTLs explained less than $20 \%$ of phenotypic variance, suggesting that other minor-effect loci remained undetected. It is also likely that hormone variations found in the RILs are partly contributed by non-genetic factors, e.g. environmental perturbation, despite all attempts to control and standardize the growth conditions of the plants.

Another reason to explain the relative low number of hormone QTLs is differential spatial distributions of hormone metabolites in distinct root tissues and/or cell types. The described auxin maximum in the root apex indicates that local biosynthesis and polar transport results in auxin gradients and differential distribution (Petersson et al., 2009). Antoniadi et al. (2015) reported that a concentration gradient and cell-type-specific distribution for CKs are present in the Arabidopsis root apex. Since hormone quantification in our study was determined from homogeneous powders of the whole root system, it is impossible to draw conclusions on differential hormone concentrations in different cell types. Such mean effects present in the root samples could influence QTL numbers and their relative contribution on LOD scores for hormones and their metabolites. It can also explain why no QTLs for levels of auxin and ABA were above the significant threshold (data not included). A way to avoid this problem-local dissection of the complex root system before extraction-would have also brought about heterogeneity of sampling due to unclear boundaries in the continuous meristemelongation-differentiation-transition zones, and the sensitivity of the detection method might have been too low, and hence why it was not done in this study.

The co-localization of QTLs for metabolite-ratios with those for single compound suggests that the underpinning gene(s) can be involved in the metabolism of either early or late pathways (e.g., at chromosome 2 and 5 in Fig 2). In addition, a newly observed QTL for the ratio between tZ7G and tZ9G in chromosome 5 would be another indication that the metabolic conversion of these $\mathrm{tZ} \mathrm{N}$ glucosides is controlled genetically, differing with the previous assertion that it resulted from either non-biological reaction or enzymatic $N$-glucosylation (Hou et al., 2004).

\section{Detection of developmental-specific QTLs from vegetative and flowering lines}

The difference of QTL profiles between vegetative and flowering sub-populations suggests that some QTLs related to hormone levels in roots are development-specific (Fig 3). The transition to flowering can trigger changes of source-sink relationships in leaves through hormone signalling (e.g., gibberellins in Arabidopsis) (Xing et al., 2015; Yamaguchi et al., 2014). Our finding of differential QTL profiles for two distinct development stages implies that the transition to flowering affects root traits and hormone contents.

Using rather mature plants provides advantages to analyse the flowering-specific loci that would not have been possible in seedlings. QTLs for specific developmental stages have been also identified 
in other studies, e.g., for linolenic acid content in soybean seed, plant height in Brassica and fruit size in cucumber (Han et al., 2011; Wang et al., 2015; Weng et al., 2015). All these studies used whole RIL populations repetitively for different developmental stages, and then a QTL for each group was re-analysed separately. Our approach can be applied to find whether an interesting QTL is temporally functional at a particular stage, especially in roots, that do not show such obvious developmental changes as seen in shoots. In the present study, numbers of significant QLTs for the three categories were $8: 5: 2$ (vegetative : flowering : non-development-specific or constant). It implies that a large number of loci for root growth and hormone levels are temporally functional rather than being continuously active for the two developmental stages.

In the vegetative lines, two QTLs were newly found that were detected neither in the whole populations, nor in the flowering lines, viz., for SA and RL. Thus, these loci are only (or mainly) active in the vegetative stage, and including data from flowering plants only adds non-genetic variation, thus obscuring the QTLs. RFW has a vegetative- and a flowering-specific QTL, but RL only has a vegetative-specific QTL, suggesting that at the flowering stage root weight is still regulated by other genetic factor, or triggered by signalling from upper parts of the plant.

\section{Pleiotropic effects of UGT76C2 gene on CK metabolism and different roles of CK glucosides in roots and leaves}

Since the two trans-zeatin N-glucosyltransferase genes (At5g05860 and At5g05870) both closely positioned to BH-144L on chromosome 5 where LOD scores of the QTL regions were highest, it is likely that one of these genes (or both) are responsible for determining levels of tZ7G and tZ9G. However, due to lack of detailed makers in the region between the end-point of Cvi introgression in NIL5-1 and the start-point of Cvi introgression in NIL5-2, it was uncertain which of the two genes caused (most of) the effect.

Pleotropic effects of UGT76C2 gene, encoding an $N$-glucosyltransferase, on many CK metabolites were found in the present study. In Arabidopsis roots, the loss-of-function of this gene greatly reduced levels of not only $\mathrm{N}$-glucosides, but also other upstream metabolites in both $\mathrm{tZ}$ pathway and other side (lateral) chains in CK metabolism. In leaves, contents of CK metabolites were less affected, except compounds in the $\mathrm{tZ}$ pathway, as also found in a recent study (Smehilova et al., 2016). Wang et al. (Wang et al., 2011) also elucidated the functional role of UGT76C2 in Arabidopsis seedling ( 2 weeks) using KO plants and overexpressors. They reported significant reductions of levels of CK $N$-glucosides but little changes of other CK metabolites. In comparison with the findings of Wang et al. (Wang et al., 2011), the observation of more extensive changes of the contents of CK metabolites in the present study may be caused by differences of organs (only roots in the present study) and age (5 weeks). Unlike $\mathrm{CK} N$-glucosides, levels of $O$-glucosides in the KO plants hardly 
changed in both studies. This is consistent with another study that two $\mathrm{N}$-glucosyltransferases ( $U G T 76 C 2$ and $U G T 76 C 1$ ) in Arabidopsis are involved in CK $N$-glucosylation, and three other UGTs are separately responsible for $O$-glucosylation (Hou et al., 2004). The reduction of CK $N$-glucoside levels resulted in significant dwarfism of root phenotypes in our study. This is consistent with the findings of Kollmer et al. (2014) showing that levels of CK $N$-glucosides positively correlated with root growth and development. However, it is difficult to evaluate to what extent the changes of levels of other CK metabolites directly affected root growth.

The decrease of levels of $\mathrm{N}$-glucosides in both roots and leaves is in agreement with transcriptomic data studied by Schmid et al. (2005), showing that the At5g05860 gene, coding for UGT76C2, is substantially transcribed in both roots and leaves at the early vegetative stage, but becomes inactive in roots during flowering. The reduction of levels of CK $N$-glucosides in leaves hardly affected shoot weight and other aerial phenotypes. This is consistent with vegetative-specificity of the QTLs for levels of $\mathrm{tZ7G}$ and tZ9G, although it is hard to conclude whether only UGT76C2 is responsible for the QTL or if other genes are also involved. Based on presented data, it is likely that $\mathrm{CK} \mathrm{N}$-glucosides have different physiological roles in roots compared to shoots since depletion of $\mathrm{N}$ glucosides only significantly affected root growth (Fig 6C).

\section{Conclusion}

In conclusion, QTL analysis based on hormone levels in Arabidopsis roots revealed genetic loci involved in regulation and/or metabolism of CK. Flowering has a profound effect on many of the QTLs detected. Differential regulations of hormone QTLs in the vegetative vs. flowering stages suggest that it may be interesting to study other RIL populations derived from late-flowering lines, in which all progenies can be in the vegetative stage at the sampling time. For a better understanding of hormone regulation at the whole-plant level, hormone-metabolic QTL studies should be extended to other organs, e.g., leaves. The loss-of-function analysis of $U G T 76 C 2$, a gene underlying a major QTL for levels of tZ7G and tZ9G suggests that CK $N$-glucosides play an important role in root development.

\section{Acknowledgements}

The authors thank Dr. Leonie Bentsink for providing seeds of Ler $\times$ Cvi RIL populations, Dr. Joost Keurentjes for NILs and AFLP map and Ms. Kaili Kim for the correction of manuscript. 


\section{[Supplementary Information]}

Table S1. Summary of multiple reaction monitor (MRM) transitions used for hormone quantification in UPLC-ESI-TQ mass spectrometer.

\begin{tabular}{|c|c|c|c|c|c|c|c|c|c|c|c|c|}
\hline Analytes & $\begin{array}{c}\text { UPLC } \\
\text { Gradient }\end{array}$ & RT & ESI & $\begin{array}{c}\text { MRM } \\
\text { Function }\end{array}$ & Transition & $\begin{array}{c}\text { Cone } \\
\text { voltage }\end{array}$ & $\begin{array}{l}\text { Collision } \\
\text { energy }\end{array}$ & $\begin{array}{l}\text { Internal } \\
\text { standard }\end{array}$ & RT & Transition & $\begin{array}{c}\text { Cone } \\
\text { voltage }\end{array}$ & $\begin{array}{l}\text { Collision } \\
\text { energy }\end{array}$ \\
\hline$\overline{\mathrm{tZ}}$ & 1 & 3.78 & + & 2 & $220.1>136.1$ & 20 & 18 & {$\left[{ }^{2} \mathrm{H}_{6}\right] \mathrm{tZ}$} & 3.75 & $225.1>136.9$ & 20 & 18 \\
\hline tZR & 1 & 5.04 & + & 3 & $352.1>136.1$ & 28 & 20 & {$\left[{ }^{2} \mathrm{H}_{5}\right] \mathrm{tZR}$} & 5.01 & $357.1>136.9$ & 28 & 20 \\
\hline iP & 1 & 6.15 & + & 1 & $204.1>136.1$ & 20 & 18 & {$\left[{ }^{2} \mathrm{H}_{6}\right] \mathrm{iP}$} & 6.11 & $210.1>137.1$ & 20 & 18 \\
\hline iPR & 1 & 7.60 & + & 1 & $336.1>204.1$ & 28 & 18 & {$\left[{ }^{2} \mathrm{H}_{6}\right] \mathrm{iPR}$} & 7.55 & $342.2>210.2$ & 28 & 18 \\
\hline$c Z$ & 1 & 4.01 & + & 2 & $220.1>136.1$ & 20 & 18 & {$\left[{ }^{2} \mathrm{H}_{6}\right] \mathrm{tZ}$} & 3.75 & $225.1>136.9$ & 20 & 18 \\
\hline CZR & 1 & 5.31 & + & 3 & $352.1>136.1$ & 28 & 16 & {$\left[{ }^{2} \mathrm{H}_{5}\right] \mathrm{tZR}$} & 5.01 & $357.1>136.9$ & 28 & 20 \\
\hline tZOG & 1 & 3.84 & + & 2 & $382.1>220.1$ & 30 & 16 & {$\left[{ }^{2} \mathrm{H}_{5}\right] \mathrm{tZ9G}$} & 4.02 & $387.2>225.2$ & 30 & 20 \\
\hline $\mathrm{tZ7G}$ & 1 & 3.86 & + & 2 & $382.1>220.1$ & 30 & 20 & {$\left[{ }^{2} \mathrm{H}_{5}\right]$ tZ9G } & 4.02 & $387.2>225.2$ & 30 & 20 \\
\hline tZ9G & 1 & 4.04 & + & 2 & $382.1>220.1$ & 30 & 20 & {$\left[{ }^{2} \mathrm{H}_{5}\right] \mathrm{tZ9G}$} & 4.02 & $387.2>225.2$ & 30 & 20 \\
\hline $\mathrm{DZ}$ & 1 & 3.95 & + & 2 & $222.1>136.1$ & 20 & 18 & {$\left[{ }^{2} \mathrm{H}_{3}\right] \mathrm{DZ}$} & 3.91 & $225.1>136.1$ & 20 & 18 \\
\hline DZR & 1 & 5.10 & + & 3 & $354.1>136.1$ & 28 & 22 & {$\left[{ }^{2} \mathrm{H}_{3}\right] \mathrm{DZR}$} & 5.06 & $357.2>149.1$ & 28 & 22 \\
\hline DZOG & 1 & 4.18 & + & 2 & $384.1>222.1$ & 30 & 16 & {$\left[{ }^{2} \mathrm{H}_{5}\right]$ tZ9G } & 4.02 & $387.2>225.2$ & 30 & 20 \\
\hline DZ7G & 1 & 4.07 & + & 2 & $384.1>136.1$ & 30 & 20 & {$\left[{ }^{2} \mathrm{H}_{5}\right] \mathrm{tZ9G}$} & 4.02 & $387.2>225.2$ & 30 & 20 \\
\hline DZ9G & 1 & 4.17 & + & 2 & $384.1>136.1$ & 30 & 20 & {$\left[{ }^{2} \mathrm{H}_{5}\right]$ tZ9G } & 4.02 & $387.2>225.2$ & 30 & 20 \\
\hline tZRMP & 1 & 3.58 & + & 2 & $432.1>220.1$ & 34 & 22 & & & & & \\
\hline IAA & 2 & 4.15 & + & 1 & $176.1>130.1$ & 18 & 16 & {$\left[{ }^{13} \mathrm{C}_{6}\right]$ IAA } & 4.14 & $182.1>136.1$ & 18 & 16 \\
\hline OxIAA & 2 & 3.62 & + & 1 & $191.9>145.9$ & 18 & 16 & & & & & \\
\hline $\mathrm{ABA}$ & 2 & 5.28 & + & 2 & $265.2>247.2$ & 18 & 14 & {$\left[{ }^{2} \mathrm{H}_{6}\right] \mathrm{ABA}$} & 5.26 & $271.2>253.2$ & 18 & 14 \\
\hline$J A$ & 2 & 6.00 & + & 3 & $211.2>133.1$ & 18 & 16 & {$\left[{ }^{2} \mathrm{H}_{4}\right] \mathrm{JA}$} & 5.98 & $216.2>135.3$ & 18 & 16 \\
\hline SA & 2 & 4.76 & - & 2 & $136.9>92.9$ & 25 & 15 & {$\left[{ }^{2} \mathrm{H}_{4}\right] \mathrm{SA}$} & 4.75 & $140.9>96.9$ & 25 & 15 \\
\hline
\end{tabular}


Table S2. Significant QTLs for hormone levels and root phenotypic traits. ${ }^{\S}$ indicates loci for traits observed by QTL analysis using only vegetative lines.

\begin{tabular}{|c|c|c|c|c|c|c|c|c|}
\hline & Trait & $\begin{array}{l}\text { Chromo- } \\
\text { some }\end{array}$ & $\begin{array}{c}\mathrm{cM} \\
\text { interval }\end{array}$ & $\begin{array}{c}\text { LOD } \\
\text { (MQM) }\end{array}$ & $\begin{array}{l}\text { Locus } \\
\text { region }\end{array}$ & $\begin{array}{l}\text { Nearest } \\
\text { marker }\end{array}$ & $\begin{array}{c}\text { Explained (\%), } \\
\text { MQM }\end{array}$ & $\begin{array}{l}\text { Additive } \\
\text { effect }\end{array}$ \\
\hline \multirow{14}{*}{$\begin{array}{l}\text { Hormone } \\
\text { compounds }\end{array}$} & iPRMP & 5 & $94.9 \sim 117$ & 2.5 & $\begin{array}{c}\text { GB.102L- } \\
\text { Col/105C HH.122C/120L }\end{array}$ & DF.199L & 8 & + \\
\hline & tZRMP & 5 & $2 \sim 14.8$ & 6.6 & FD.207L EC.198L-Col & $\mathrm{CH} .690 \mathrm{C}$ & 16 & - \\
\hline & tZR & 5 & $95.9 \sim 116$ & 2.7 & $\begin{array}{c}\text { GB.102L- } \\
\text { Col/105C HH.122C/120L }\end{array}$ & DF.119L & 8 & - \\
\hline & \multirow{3}{*}{ tZOG } & 2 & $45.6^{\sim} 51.2$ & 11.7 & FD.150C GD.298C & Erecta & 24 & + \\
\hline & & 3 & $0 \sim 5.8$ & 4.1 & DF.77C EG.75L & DF.77C & 7 & - \\
\hline & & 4 & $39.6 \sim 80.2$ & 3.1 & $\begin{array}{c}\text { CD.84C- } \\
\text { Col/85L BH.342C/DHS1 }\end{array}$ & CH.70L/71C-Col & 5 & - \\
\hline & tZ7G & 5 & $1 \sim 11.6$ & 8.0 & FD.207L EC.198L-Col & BH.144L & 20 & + \\
\hline & \multirow{3}{*}{ tZ9G } & 1 & $0 \sim 30.9$ & 2.9 & PVV4 CC.98L-CH.160L-Col & PVV4 & 6 & - \\
\hline & & 3 & $0 \sim 5.8$ & 5.2 & DF.77C EG.75L & DF.77C & 11 & - \\
\hline & & 5 & $5.3 \sim 10.6$ & 11.0 & CH.690C EC.198L-Col & BH.144L & 25 & + \\
\hline & \multirow{2}{*}{ DZOG } & 1 & $16.3 \sim 46.7$ & 2.9 & EC.480C $\sim$ GB.112L & CH.160L-Col & 7 & - \\
\hline & & 1 & $52.1 \sim 67$ & 3.3 & GB.112L CD.89C & GD.97L & 9 & + \\
\hline & $\mathrm{JA}$ & 3 & $69.3 \sim 85.8$ & 3.3 & FD.98C $\mathrm{HH} .90 \mathrm{~L}-\mathrm{Col}$ & HH.171C-Col/173L & 9 & - \\
\hline & SA & $1^{\S}$ & $35.7^{\sim} 56.1$ & 3.6 & CC.98L-Col/101C GD.97L & AD.106L-Col & 20 & + \\
\hline \multirow{7}{*}{$\begin{array}{c}\text { Ratios } \\
\text { (substrate- } \\
\text { to- product) }\end{array}$} & iPRMP/tZRMP & 5 & $0 \sim 10.6$ & 5.9 & FD.207L EC.198L-Col & BH.144L & 17 & + \\
\hline & tZRMP/tZR & 5 & $2 \sim 33.1$ & 3.6 & FD.207L DF.184L-Col & EC.198L-Col & 10 & - \\
\hline & $\mathrm{tZ} / \mathrm{tZOG}$ & 2 & $38.4 \sim 51.2$ & 6.3 & FD.85C GD.298C & Erecta & 16 & - \\
\hline & tZ/tZ7G & 5 & $0 \sim 15.8$ & 3.0 & FD.207L BH.107L-Col & BH.144L & 8 & - \\
\hline & $\mathrm{tZ/tZ9G}$ & 5 & $0 \sim 15.8$ & 3.0 & FD.207L BH.107L-Col & BH.144L & 9 & - \\
\hline & $\mathrm{tZ7G/tZ9G}$ & 5 & $62.5^{\sim} 81.5$ & 3.3 & BH.96L-Col AD.75C-Col & CD.116L & 10 & - \\
\hline & tZOG/DZOG & 2 & $44.6^{\sim} 51.2$ & 7.0 & FD.85C GD.298C & Erecta & 19 & + \\
\hline \multirow{6}{*}{$\begin{array}{l}\text { Phenotypic } \\
\text { traits }\end{array}$} & $\mathrm{RL}$ & $5^{\S}$ & $9.6 \sim 36.8$ & 3.2 & BH.144L GH.117C & BH.107L-Col & 16 & - \\
\hline & \multirow{2}{*}{ RFW } & 1 & $2 \sim 15.1$ & 7.3 & PVV4 EC.480C & HH.335C-Col/PhyA & 16 & - \\
\hline & & 5 & $93 \sim 117$ & 4.3 & CC.262C HH.122C/120L & DF.119L & 10 & + \\
\hline & \multirow{2}{*}{ SFW } & 1 & $0 \sim 27.3$ & 5.6 & PVV4 CH.160L-Col & PVV4 & 14 & - \\
\hline & & 2 & $61.2^{\sim} 70.8$ & 3.2 & BH.120L-Col EC.235L-Col & EC.235L-Col & 7 & + \\
\hline & $\begin{array}{c}\text { Flowering, stalk } \\
\text { number }\end{array}$ & 1 & $0 \sim 10$ & 15.8 & PVV4 HH.335C-Col/PhyA & PVV4 & 34 & - \\
\hline
\end{tabular}



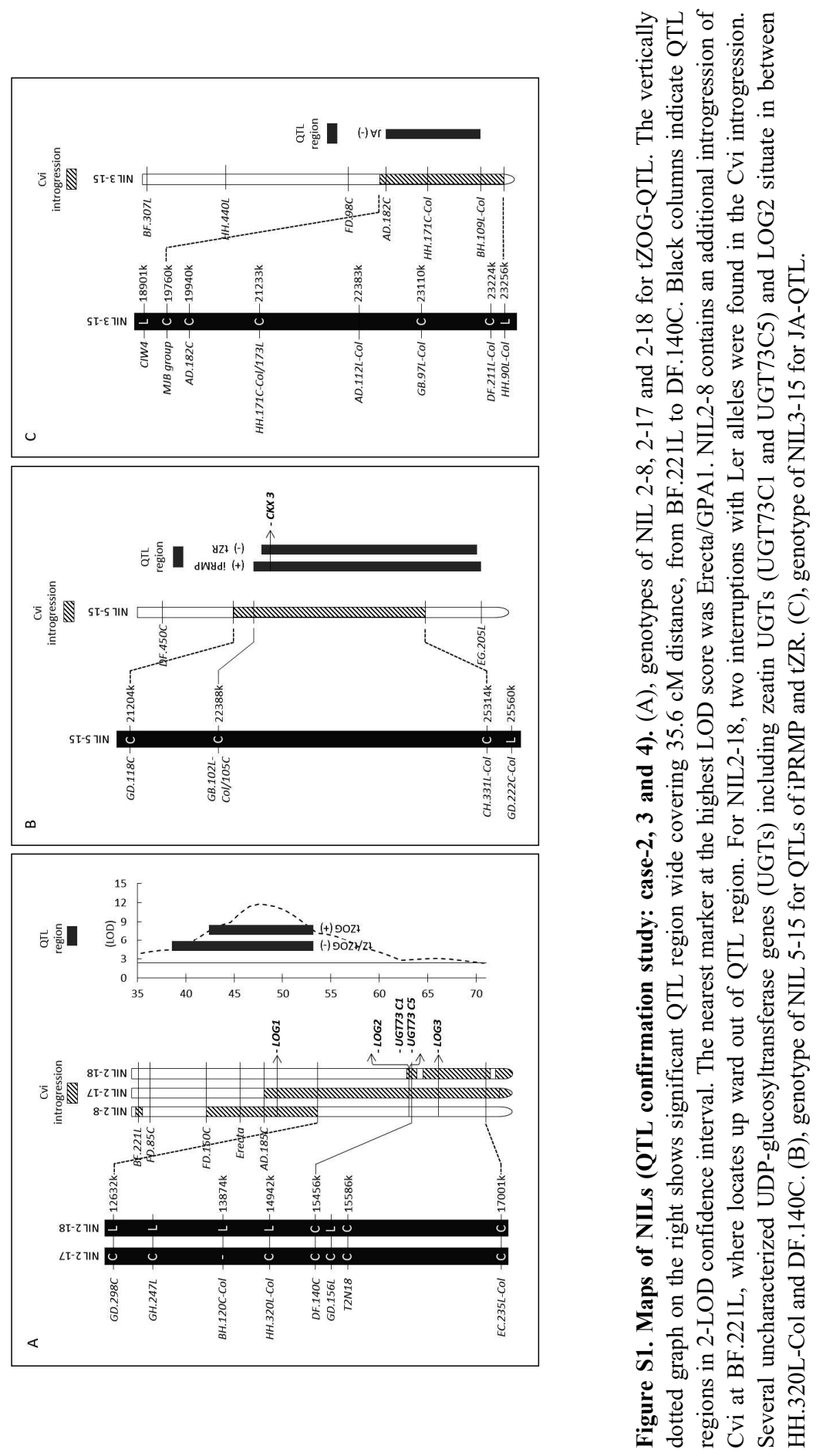


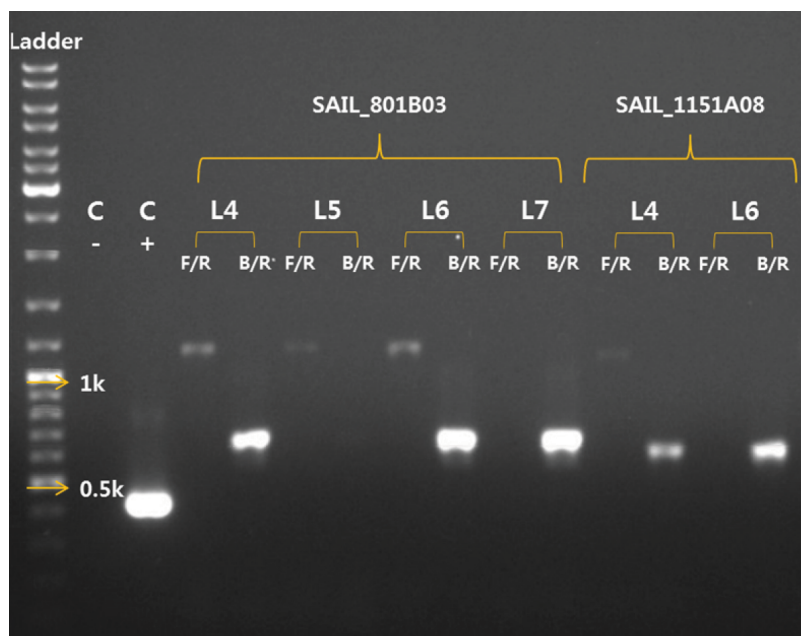

Figure S2. Homozygosity test of two KO lines (SAIL 801B03 and SAIL 1151A08). The genotyping of TDNA insertion was based on a comparison between two PCR reactions: a set of the forward and the reverse primer in the gene, F/R; a set of Bp (BPos in T-DNA) and the reverse primer of the gene, B/R. For SAIL 801B03, Line 4, Line5 and Line 6 were heterozygous, but Line 7 was homozygous, which was chosen for the comparison test between the wild type and the KO plant (Fig 6B). For SAIL 1151A08, Line 6 was homozygous, but significant changes of levels of tZ $N$-glucosides were not observed (S3 Fig) because T-DNA insertion might be taken place in the intron region (Fig 6A).

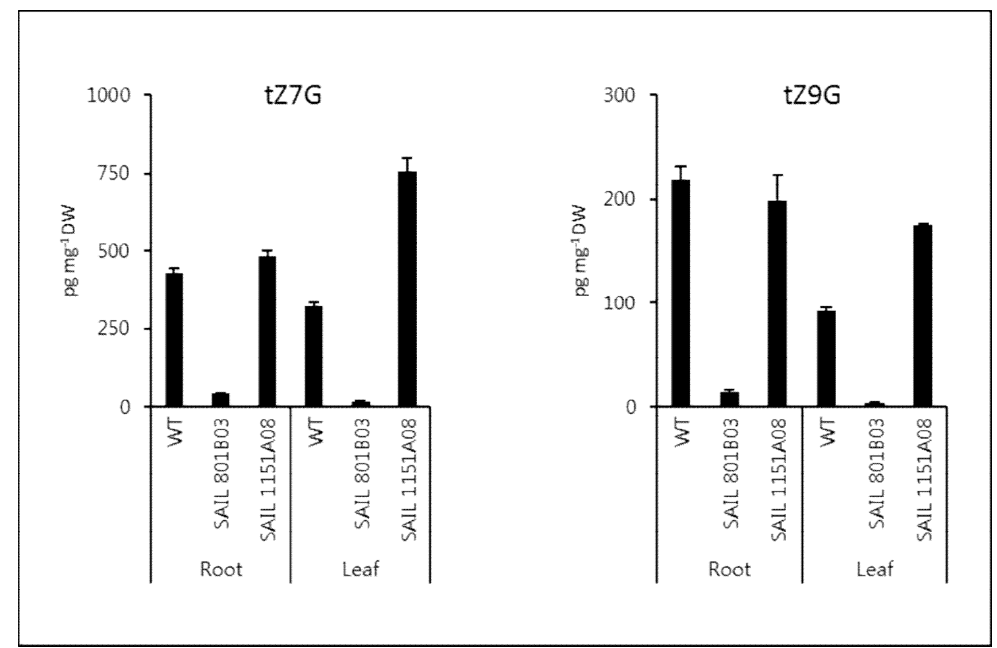

Figure S3. Levels of tZ7G and tZ9G in two KO lines for At5g05860. 



\section{Chapter 5}

\section{Quantitative trait loci for hormone levels in Arabidopsis leaves}

Sangseok Lee, Lidiya I. Sergeeva, Harro J. M. Bouwmeester and Dick Vreugdenhil 


\begin{abstract}
Linkage mapping analysis is a powerful tool to analyse complex traits, such as plant hormone levels. In this study, 5 groups of hormones were quantitatively analysed in the leaves of an Arabidopsis recombinant inbred line (Ler $\times \mathrm{Cvi})$ population using tandem mass spectrometry coupled with liquid chromatography. QTL analysis showed a multitude of significant loci for levels of various hormones, viz., IAA, ABAs and CKs. Development-specific QTLs were detected in two sub-populations, vegetative and flowering lines. QTLs for ratios between hormone intermediates belonging to the same class and also to different classes were found. Many of these ratio-QTLs co-localized with QTLs for one of the hormones in the pairs and additionally several new QTL regions were found. This suggests that hormone balances may partly be controlled by new specific loci and partly by loci for the single hormone compounds. The present results provide new insight towards the understanding of the genetic basis of hormone metabolism in plants.
\end{abstract}

Key words: Arabidopsis thaliana, quantitative trait loci (QTLs), plant hormones, recombinant inbred lines (RILs), leaves 


\section{Introduction}

Quantitative trait loci (QTL) analysis has been deployed to unravel genomic regions that are responsible for diverse phenotypic traits of interest in a wide range of organisms. In plants, the identification of genes affecting polygenic traits has increased enormously over the last decades (Alonso-Blanco et al., 2009). Linkage mapping analysis has been applied not only to visible developmental and physiological traits (e.g. flowering time and dormancy), but also to biochemical properties of plants (Alonso-Blanco and Mendez-Vigo, 2014; Feng et al., 2012; Kliebenstein, 2009). The integration of QTL results, obtained at molecular and phenotypic levels may lead to a better understanding of plant-environment interactions. For instance, in Arabidopsis, QTL analysis of glucosinolates led to the identification of epithospecifier modifier-1 (ESM1), a gene controlling glucosinolate hydrolysis that induces structural diversity and herbivore resistance (Pfalz et al., 2009; Zhang et al., 2006).

Plant hormones play important roles in growth and development as internal messengers at very low quantities. Hormone contents, as well as sensitivities towards hormones, are likely to be polygenic traits. However, the quantitative regulation of levels of plant hormones has hardly been studied despite their significant roles in plant development. In Arabidopsis, plant hormone levels are quantitative traits varying among natural accessions in roots (Lee et al., 2017). Our previous QTL study (Lee et al., 2019) on roots using recombinant inbred lines (RILs) of Arabidopsis revealed several significant QTLs for different groups of hormones. Co-localization of QTLs for cytokinin (CK) metabolites suggested that various hormones can be regulated by a common molecular pathway resulting in pleiotropic effects. For the level of a single hormone metabolite, e.g., tZ9G, multiple loci were involved and many loci were development-specific. This study on roots shows the feasibility of QTL mapping of hormones in plants, and hence raises the question about the presumed polygenic regulation of hormones in above-ground organs, further allowing us to compare hormone QTLs in leaves with those found in roots.

Within plants, hormones are translocated by various transport systems from biosynthetic sites to distant organs. Long-distance transport of hormones is achieved by distinct hormone transporters through the vascular system (Galweiler et al., 1998; Ko et al., 2014; Lacombe and Achard, 2016). Metabolic conversion may occur along the transport pathway, for example in the xylem, where ratios between precursors and products, e.g., tZR and tZ, may depend on environmental conditions (Kang et al., 2015; Osugi et al., 2017). It is likely that multiple genetic components regulate hormone content and that there are differences between roots and leaves, but to our knowledge this has not been studied through quantitative genetic analysis. 
In this study, we conducted quantitative analysis of five groups of plant hormones (auxin, ABA, $\mathrm{CK}$, salicylic acid (SA), and jasmonic acid (JA)) including some metabolites of ABA and CK, in rosette leaves of Ler $\times$ Cvi recombinant inbred lines (RILs) of Arabidopsis. We asked which genetic loci are associated with hormone levels in leaves and how these loci can be differentially functional compared with those for hormones in roots. In order to compare correlations between hormones and phenotypic traits and between two different organs, we combined the quantitative data of hormones in leaves with those in roots obtained from our previous study using the same RIL population. Significant correlations between levels of various hormones were only observed within the same tissue, but not between the two distant organs. However, for CKs several QTLs in leaves co-localized with those in roots. According to developmental stages at the time of sampling, the RIL lines could be divided into two sub-populations, vegetative and flowering lines, resulting in development-specific QTLs. Comparative analyses of hormone QTLs between leaves and roots and between different hormones may provide a comprehensive understanding of how plants coordinate hormone levels in different organs and during various development stages.

\section{Materials and Methods}

\section{Plant materials and phenotyping}

Ler $\times$ Cvi RILs, developed and genotyped by Alonso-Blanco et al. (1998b), were chosen based on our study on natural variation of hormone levels in roots of Arabidopsis accessions (Lee et al., 2017). Seeds of 149 lines were placed on wet filter paper in a Petri-dish at $4{ }^{\circ} \mathrm{C}$ for 4 days in darkness and subsequently sown on the top of a $0.5 \mathrm{~mL}$ cylindrical plastic tube, from which the bottom had been cut off, and that was filled with $0.5 \%$ agar in half strength Hoagland's nutrient solution ( $\mathrm{pH} 5.5$ ). Seedlings were grown in hydroponic containers ( 70 plants per 10 litres, renewing the nutrient solution once a week). Plants were grown at $21^{\circ} \mathrm{C}$ during the light period $(10 \mathrm{~h})$ and at $18^{\circ} \mathrm{C}$ during the dark period (14 h). Light intensity and humidity were fixed at $125 \mu \mathrm{Mol} \mathrm{m}^{-2} \mathrm{~s}^{-1}$ and $70 \%$ respectively. After 35 days of culture, two largest rosette leaves per plant were chosen and the leaves of 4 plants were pooled for a replicate. On the day of harvest, the developmental stage (vegetative or flowering) of each line was recorded. If any of the replicates within a line showed visible bolting, the line was scored as 'flowering'.

\section{Hormone extraction and purification}

For each RIL, a replicate was used to extract endogenous hormones and further analysed for hormone quantification. For parental lines, five biological replicates were used. Powder of lyophilized leaves $(2.5 \mathrm{mg}$ ) was extracted and purified with the same methods used in our previous study (Lee et al., 2017). 


\section{Quantitative analysis of plant hormones}

For the hormone quantification, the conditions of UPLC tandem mass spectrometry were the same as those of our previous study (Lee et al., 2017, 2019). For CKs, ten microliter of sample was injected onto an Acquity UPLC HSS column $(50 \times 2.1 \mathrm{~mm}, 1.8 \mu \mathrm{m}$; Waters $)$ and eluted by binary mobile phases, A (15mM ammonium formate, $\mathrm{pH} 4.0)$ and $\mathrm{B}$ (100\% methanol), with at constant flow rate $(0.25 \mathrm{ml} \mathrm{min}-1)$ at $40^{\circ} \mathrm{C}$ for $14 \mathrm{~min}$. The linear gradient elution was performed as follows: $0 \sim 0.01 \mathrm{~min}$, $10 \%$ eluent $\mathrm{B}$; $0.01 \sim 8.0 \mathrm{~min}, 10$ to $50 \%$ eluent $\mathrm{B}$; 8.0 8.5 min, 50 to $100 \%$ eluent $\mathrm{B}$; $8.5 \sim 9.5 \mathrm{~min}$, $100 \%$ eluent B; 9.5 10.5 min, 100 to $10 \%$ eluent B. At the end of gradient, the column was equilibrated to initial conditions for $3.5 \mathrm{~min}$. Instrumental conditions for ESI-tandem mass spectrometer were same as our previous study (see Methods and Table S1 in Chapter 4).

\section{QTL analysis}

QTL analysis was performed using MapQTL software (Ooijen, 2004) as described by Bentsink et al. (2003). A LOD score threshold of 2.5 was applied to declare a significant QTL, which corresponds to a general genome-wide significance of $\mathrm{P}<0.05$, as determined by permutation tests ( 1,000 repetitions). For the purpose of revealing developmental stage-specific QTLs, all RILs were sorted into two groups according to the transition of plants for flowering at day 35, i.e., the day of sampling. For each trait in the two groups, QTL regions were independently determined by MQM analysis with automated suggested cofactors.

\section{Results}

\section{Hormone levels in leaves of Ler $\times$ Cvi RIL population}

Seventy-six lines $(51 \%)$ of the Ler $\times$ Cvi RIL population were in the flowering-stage at the time of harvest, which was the same as the flowering rate of $51 \%$ in the previous experiment for hormone QLT analysis in roots. In total 22 hormones including $15 \mathrm{CK}$ - and 4 ABA-metabolites were quantified in the leaves of 35-day-old plants of the RIL population (Figure 1). For CKs, levels of monophosphates and glucosides were strikingly higher than those of ribosides and free bases, as also reported in a previous study (Bielach et al., 2012). The level of $t Z$, the biologically most active CK was the lowest, followed by iP and cZ, which are both less active. Within the group of CK glucosides, the concentrations of $\mathrm{tZ} \mathrm{N}$-glucosides (tZ7G and tZ9G) were the highest. For ABAs, the level of ABA was lower than those of three ABA metabolites but fold-differences between RIL lines were not as large as those of CK metabolites. 


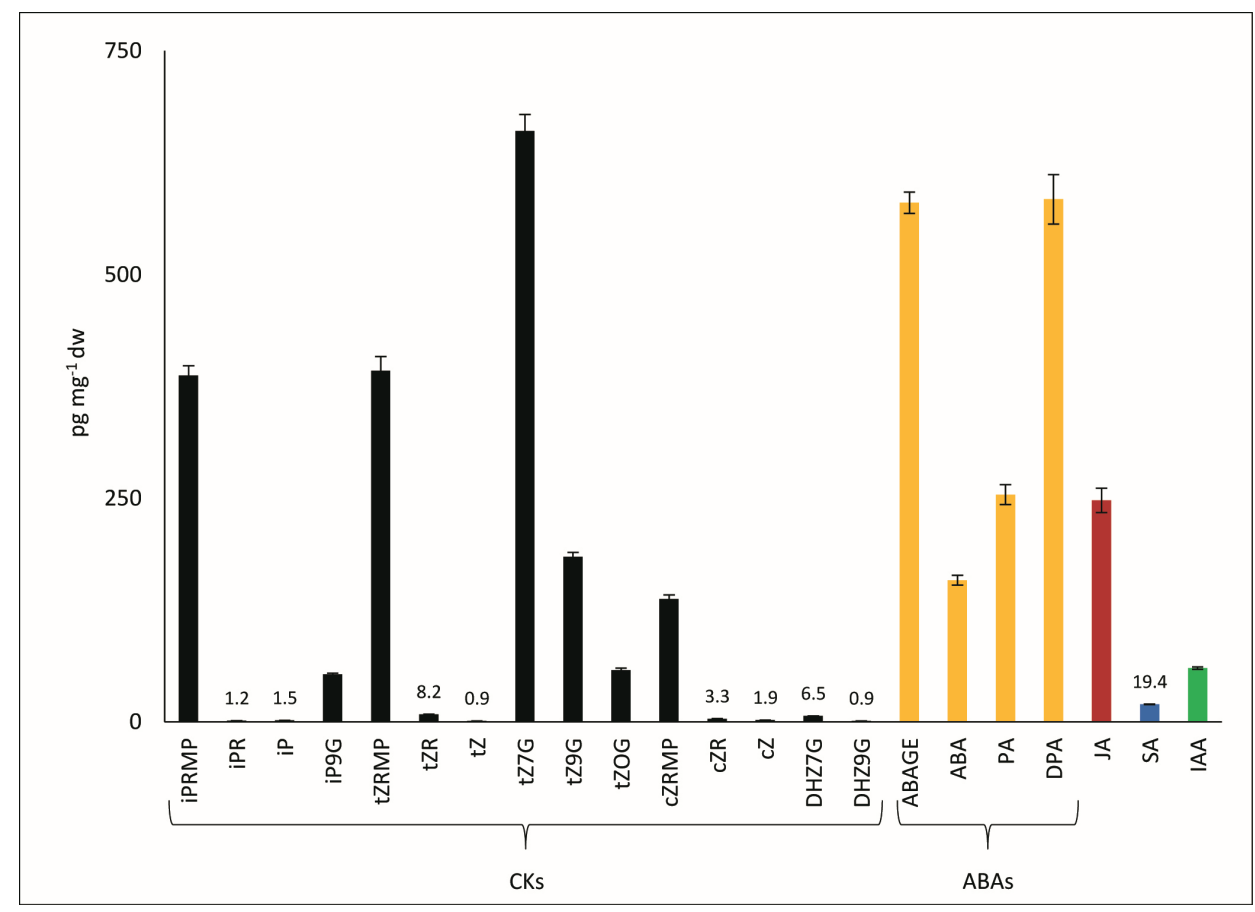

Figure 1. Levels of hormones in 35-days-old leaves of Ler×Cvi RILs. Data are averages observed in 149 lines of the population; Vertical bars on columns indicate standard errors.

Despite the small variations of hormone levels in the parental lines, large variations were found among the RILs, showing that transgressive segregation of hormone levels is present in the population (Figure 2). These variations ranged from 3-fold (ABAGE) to 160-fold (tZRMP) between extremes. Except for a few hormones, most frequency distributions were skewed towards the higher concentrations.

\section{Correlations between hormones}

For CKs and ABA, correlations between metabolites within the same hormone group were analysed (Table 1). For the CKs, significant correlations were observed between metabolites in the main pathways and the side branches (Table 1; Figure 3). Significantly positive correlations were observed between $\mathrm{tZ}$ and its glucosides. CK glucosides were significantly correlated with each other, but significantly negative correlations were observed between cZRMP and ribosides (iPR, tZR) in other side branches. Significant positive correlations were observed between ABA and its metabolites, of which dihydrophaseic acid (DPA) had the highest correlation with ABA. These correlations suggest that the inactivation of ABA by conjugation (to ABAGE) and catabolism (to PA and DPA) is controlled by a common regulatory mechanism. 

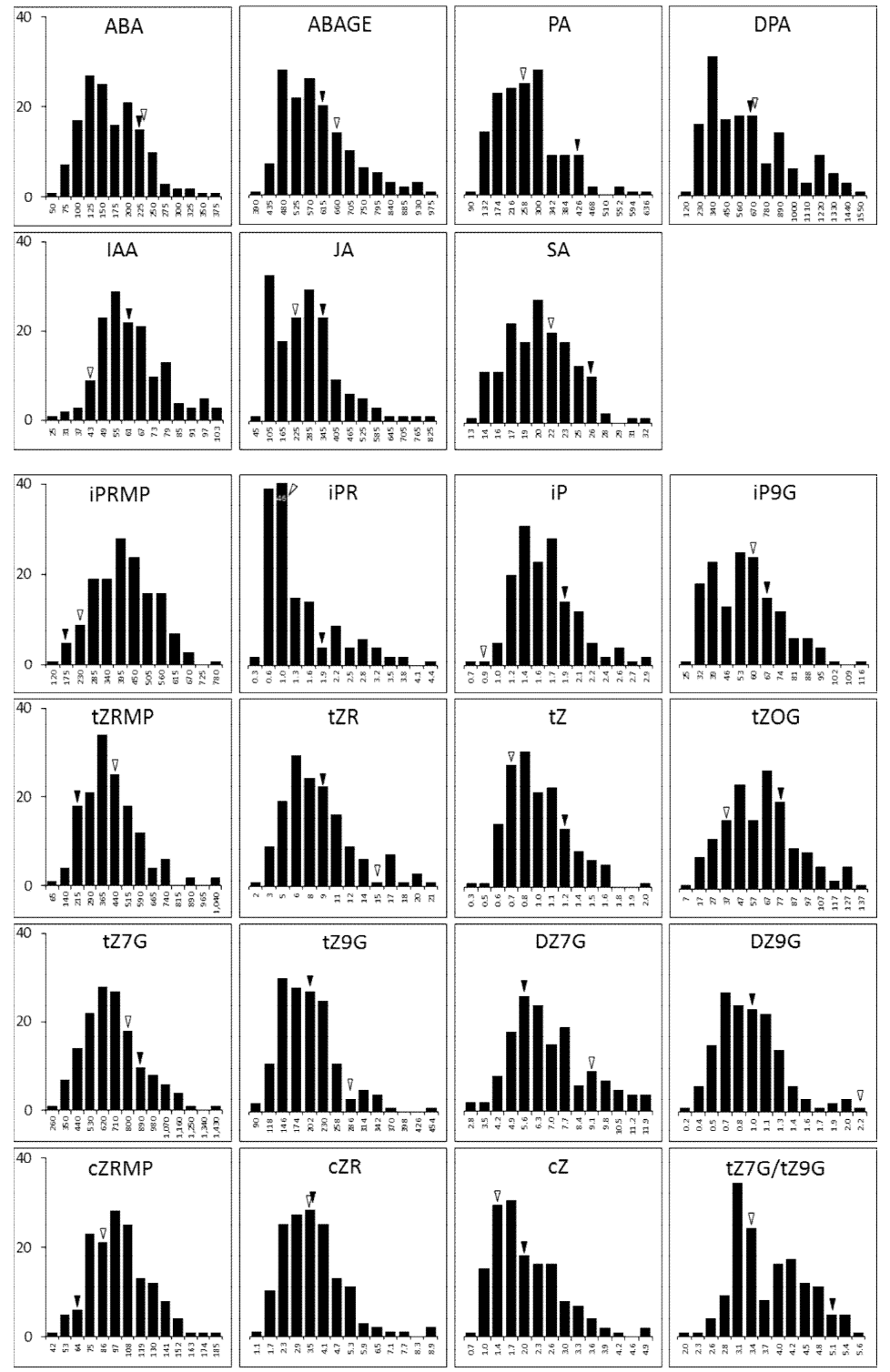

Figure 2. Frequency distributions of hormone metabolites in rosette-leaves of Arabidopsis Ler $\times$ Cvi RI ( $x$ axes: $\mathrm{pg} \mathrm{mg}^{-1} . \mathbf{d w}, y$ axes: numbers of line). Abbreviations for hormone compounds are: ABA, abscisic ac ABAGE, ABA glucose ester; PA, phaseic acid; DPA, dihydrophaseic acid; IAA, indole-3-acetic acid; J jasmonic acid; SA, salicylic acid; iPRMP, isopentenyl riboside monophosphate; iPR, isopentenyladeni riboside; iP, isopentenyladenine; iP9G, isopentenyladenine-9-glucoside; tZRMP, trans-zeatin ribosi monophosphate ; tZR, trans-zeatin riboside; tZ, trans-zeatin; tZOG, trans-zeatin-O-glucoside; tZ7,9G, trar zeatin-7,9-glucoside; DZ7,9G, dihydrozeatin-7,9-glucoside; cZRMP, cis-zeatin riboside monophosphate; cZ cis-zeatin riboside; cZ, cis-zeatin. 
Table 1. Correlations of levels of various hormone metabolites within classes of CKs and ABA, as determined in leaves of Arabidopsis Ler $\times$ Cvi RILs. Significant correlations $(p<0.05)$ are marked with an asterisk and bold figures. Blue and red colours indicate negative and positive correlations, respectively. Correlations between $\mathrm{ABA}$ and its metabolites are given separately in the upper right corner.

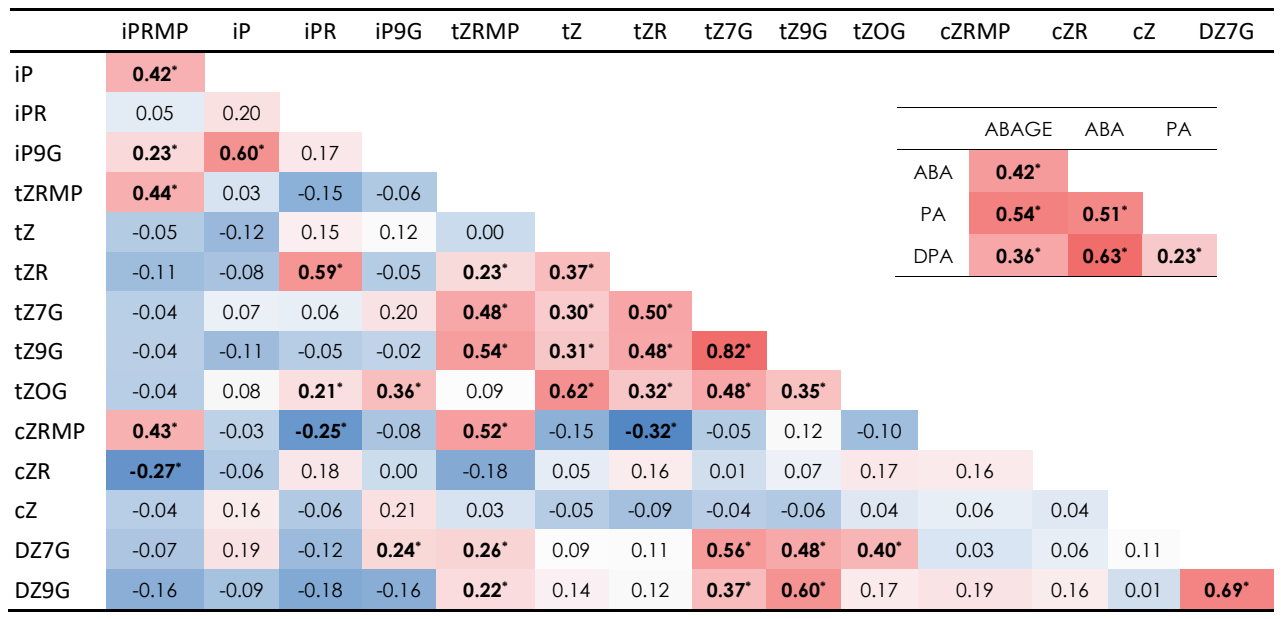

\section{QTLs for hormonal metabolites and their developmental-specificities}

The levels of the 22 hormones belonging to 5 different groups as analysed in the entire RIL population were used for QTL analysis. Among these hormones quantified in the leaves, 18 showed one or multiple significant QTLs. The average number of significant QTLs per compound was close to 3.5. No significant QTLs for JA or SA were detected. In some cases, QTLs for a hormone metabolite co-localized with those for metabolites in the same hormone group or for different kind of hormones (Figure 4, 5). For example, the QTL region on the top of chromosome 3 (DF.77C $\sim$ EG.75L) was responsible for the levels of IAA, ABA and ABA catabolites. It implies that such QTLs might be responsible for determining levels of multiple hormones.

In our previous QTL study for root hormones using the same RIL population, developmentspecific QTLs were found in sub-populations, viz., vegetative vs. flowering lines. We therefore questioned whether QTLs for leaf hormones are also development-specific. For this analysis, the whole population was split into two sub-populations, viz., vegetative and flowering lines. In many cases, QTLs detected in the whole population consisted of multiple QTLs with different developmental specificities. Interestingly, several QTLs that were not detected in the whole population were newly detected when sub-populations were used for analyses. 


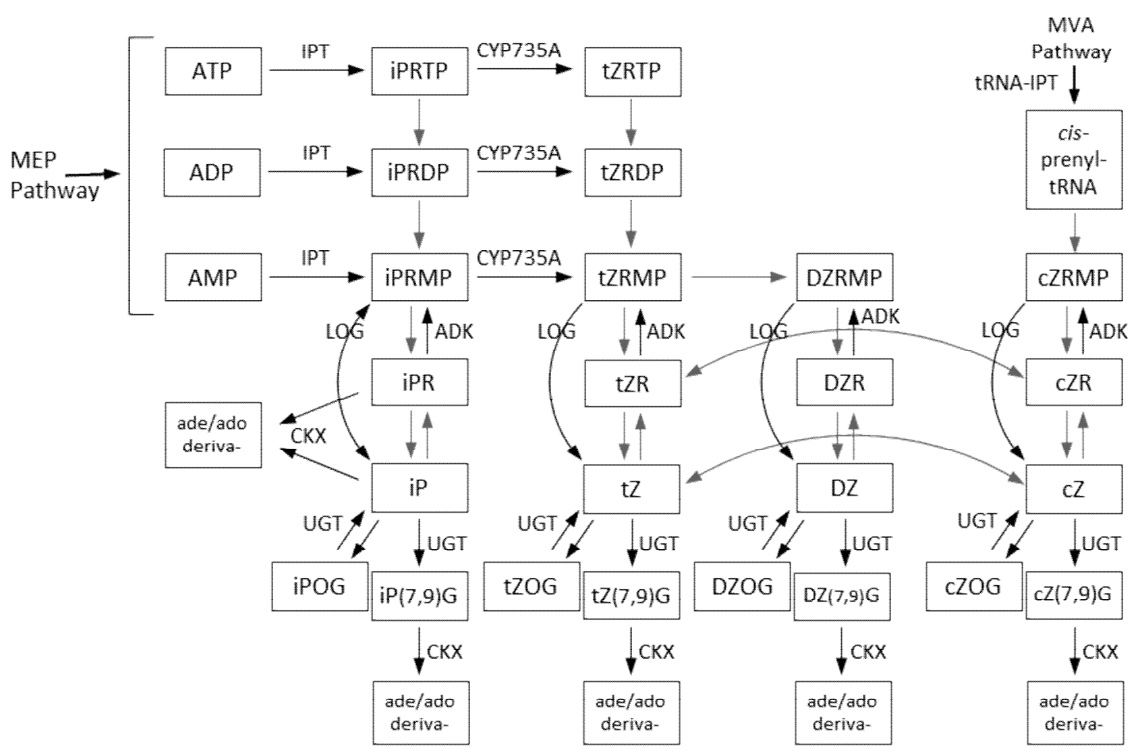

Figure 3. Scheme of CK pathways in higher plants. The pathways were adopted from review papers written by Kamada-Nobusada and Sakakibara (2009) and Werner and Schmulling (2009) with modification. Vertical arrows describe linear pathways, for example, from iPRMP to iPR to iP to iPGs. Horizontal arrows describe the pathways of side chain, for example, from iPRMP to tZRMP to DZRMP. Dark and gray arrows represent the metabolic flow with known genes and the possible pathway of unknown genes, respectively. Abbreviations of biosynthetic enzymes are: IPT, adenosine phosphate-isopentenyltransferase; CYP375A, cytochrome P450 monooxygenase; ADK, adenosine kinase; LOG, cytokinin riboside 5'-monophosphate phosphoribohydrolase (Lonely Guy); UGT, cytokinin glucosyltransferase; CKY, cytokinin oxidase/dehydrogenase.

\section{a. Development-specific QTLs for IAA and ABA metabolites}

Figure 4 shows positions of significant QTLs for the levels of IAA and 4 ABA-metabolites (see Table S5 for details).

In the whole population, two significant QLTs for IAA were found, which were present also in the vegetative stage. Three additional significant QTLs for IAA were detected only for the flowering lines.

In total, 13 QTLs for ABA and its metabolites were detected. Five of them were for ABA and the strongest QTL was located at the top of chromosome 3, explaining $11.5 \%$ of the phenotypic variance. In this region, co-localized QTLs for PA and DPA were found as well. Two QTLs for ABAGE on chromosome 1 were vegetative- and flowering-specific, respectively. Five significant QTLs for DPA were observed, explaining 3 to $22 \%$ of the phenotypic variance. Two of them were functional for the vegetative stage and one for the flowering stage. 


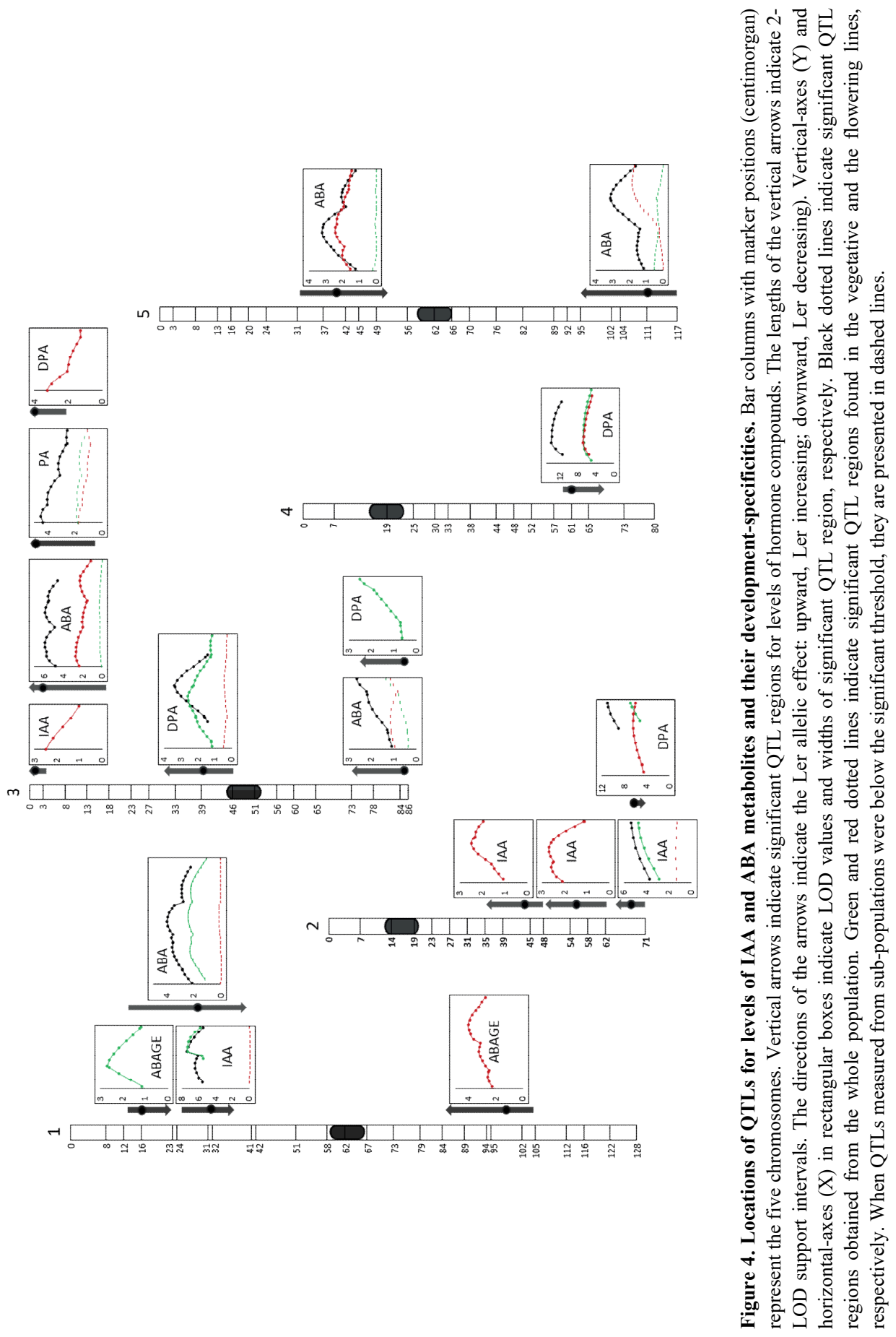



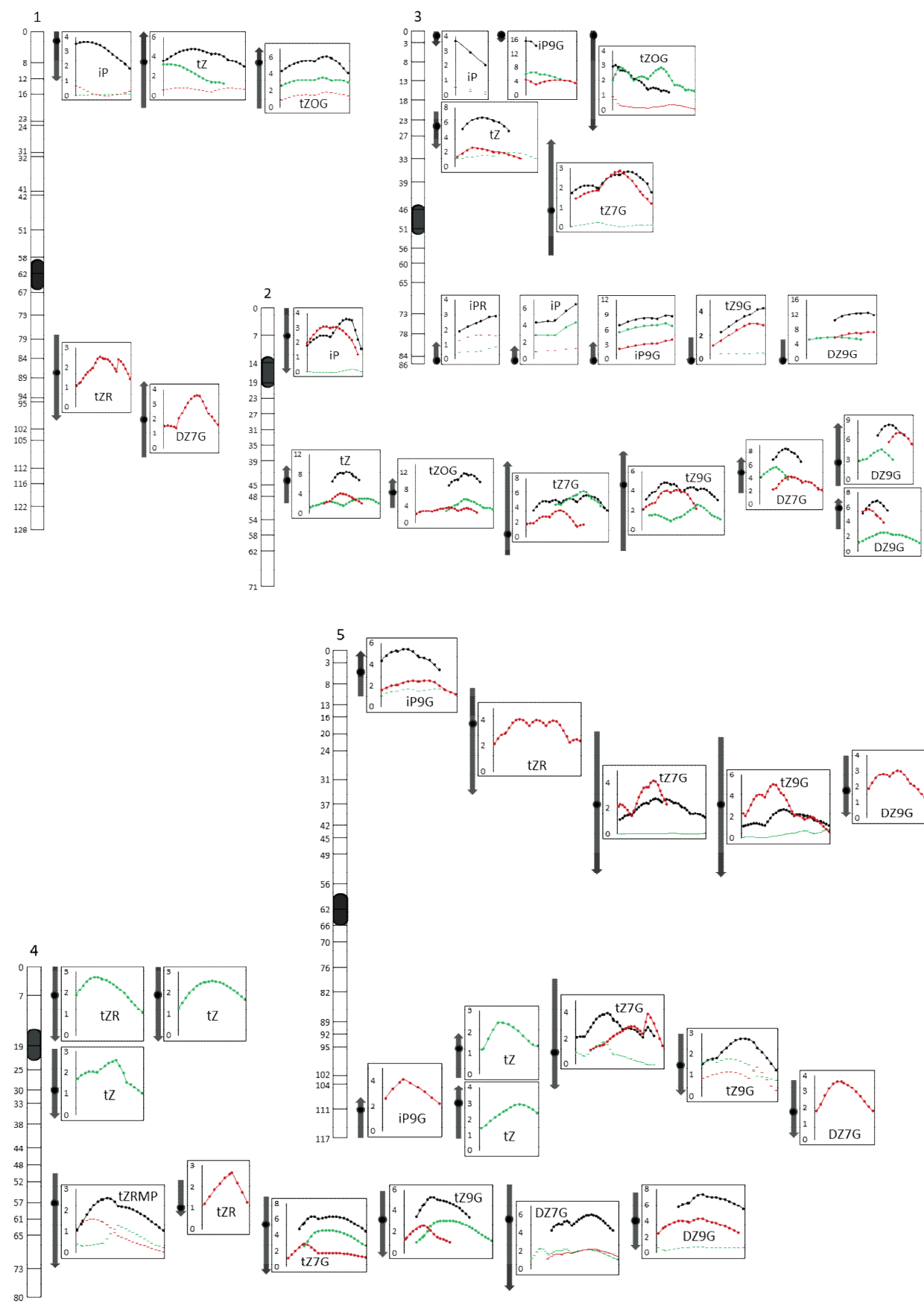

Figure 5. Locations of QTLs for levels of CK metabolites in Arabidopsis leaves and their developmentspecificities. For details, see legends of Fig 4. 


\section{b. Development-specific QTLs for CK metabolites}

The different pathways of CK metabolism are partially inter-connected, as shown in Figure 3 (Kamada-Nobusada and Sakakibara, 2009; Werner and Schmulling, 2009). In our experiments, we found more significant QTLs for $\mathrm{tZ}$ and its metabolites than for other CK metabolites, such as iP and its metabolites (Table S6). This shows that more genetic regulators for levels of $\mathrm{tZ}$ and its metabolites are present in this population compare with those in other pathways.

Figure 5 shows the locations of significant QTLs for levels of CK metabolites. Each of the CK metabolites had one or multiple loci, explaining 4 up to $30 \%$ of the phenotypic variance. In many cases, individual QTLs for different CK metabolites co-localized or partially overlapped with each other, indicating that a single locus may affect (part of) the entire pathway rather than the level of a single metabolite. In some of the co-localized regions, QTLs found in the whole population were composed of different development-specific QTLs. For instance, at the lower arm of chromosome 2, QTLs for $\mathrm{tZ}$ and CK glucosides comprised vegetative- and flowering-specific QTLs, as detected in sub-populations. Detection of such QTLs shows that multiple and complex genetic components may underlie a single QTL. These development-specificities were also found at other QTLs for both CK $\mathrm{N}$-glucosides in chromosome 4 and 5. A possible explanation might be that the QTL region contains more than one regulating locus, or that the effect of the locus on the various metabolites is development-specific, probably in interaction with other loci.

It is noteworthy that two QTLs, responsible only for $9 \mathrm{~N}$-glucosides (and not for $7 \mathrm{~N}$-glucosides) were observed on chromosome 3, one for iP9G at the top and the other for iP9G, tZ9G and DZ9G at the bottom of the same chromosome. Similarly, several QTLs only responsible for $7 \mathrm{~N}$-glucosides were found at chromosomes 1 and 3. Taken together, it is conceivable that within the $N$-glucosides, specific loci play a role in determining levels of $7 \mathrm{~N}$ - or $9 \mathrm{~N}$-glucosides separately.

\section{QTLs for metabolic ratios in precursor-product relationships}

The flux through a metabolic pathway is regulated by external and internal factors, often including the metabolites themselves in feedback regulations (Farre et al., 2014; Li et al., 2014). Illig et al. (2010) and Suhre et al. (2011) found that the use of metabolite-ratios in human biochemical phenotypes increased the statistical robustness of genome-wide association studies and detected QTLs in nearly all cases directly related to the biochemical function of an enzyme or transporter gene. Therefore, we questioned whether QTLs for metabolites-ratios can be found, by choosing precursor-product related compounds. Intriguingly, several QTLs for metabolite-ratios in the ABA and CK pathways were found (Figure 6, 7). In most cases, these QTLs co-localized with QTLs for single compounds in the analysed precursor-product pair. However, not all QTLs for metabolite ratios were at the same position as the QTL for the single compound. Interestingly, two QTLs were newly found for 
iPRMP/tZRMP and iPR/tZR at chromosome 2 and 5, where a QTL for the single compounds of these pairs was not detected.

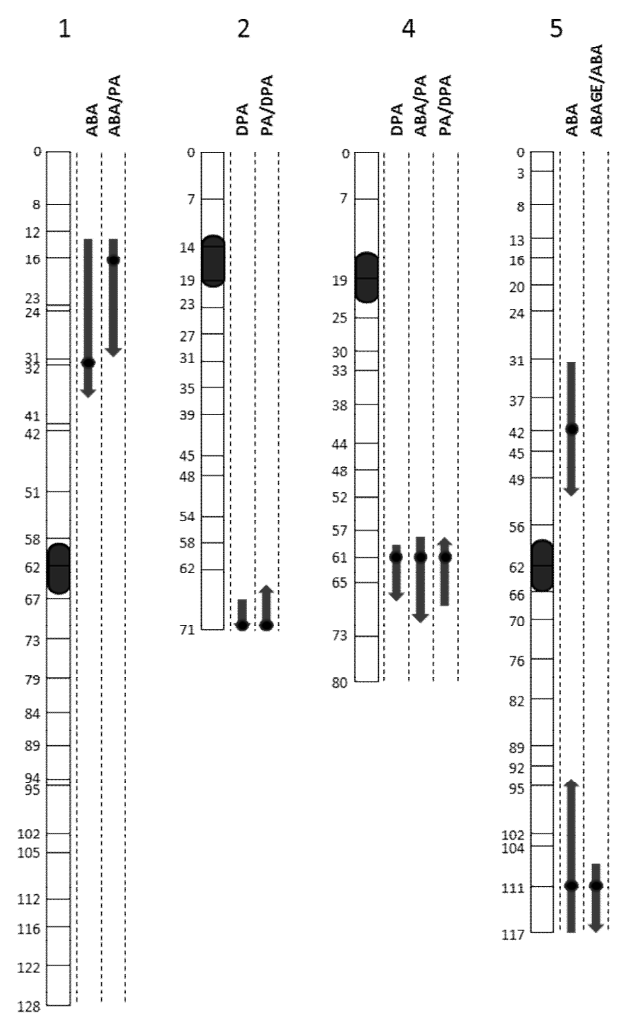

Figure 6. Significant QTLs for precursor-product ratios within ABA metabolites in Arabidopsis leaves.

\section{QTLs for hormone-hormone ratios in relationship of different groups of hormone}

Plant hormone responses are the result of complex networks of interactions involving multiple hormones, viz., hormone crosstalk (Chandler, 2009). Molecular mechanisms are likely to (partly) control such hormone interactions. Therefore, we speculated that genetic component involved in hormone crosstalk may be detected as QTLs for traits of hormone-hormone ratios between different groups of hormone. Interestingly, several loci corresponding with these kinds of trait were found (Figure 8A, Table S9). Most of the QTL were co-localized with those for the single compounds in pairs. Among these QTL regions, some of them were responsible for multiple hormones, e.g., IAAABAs-SA-JA, which were located at the bottom of chromosome 2. 

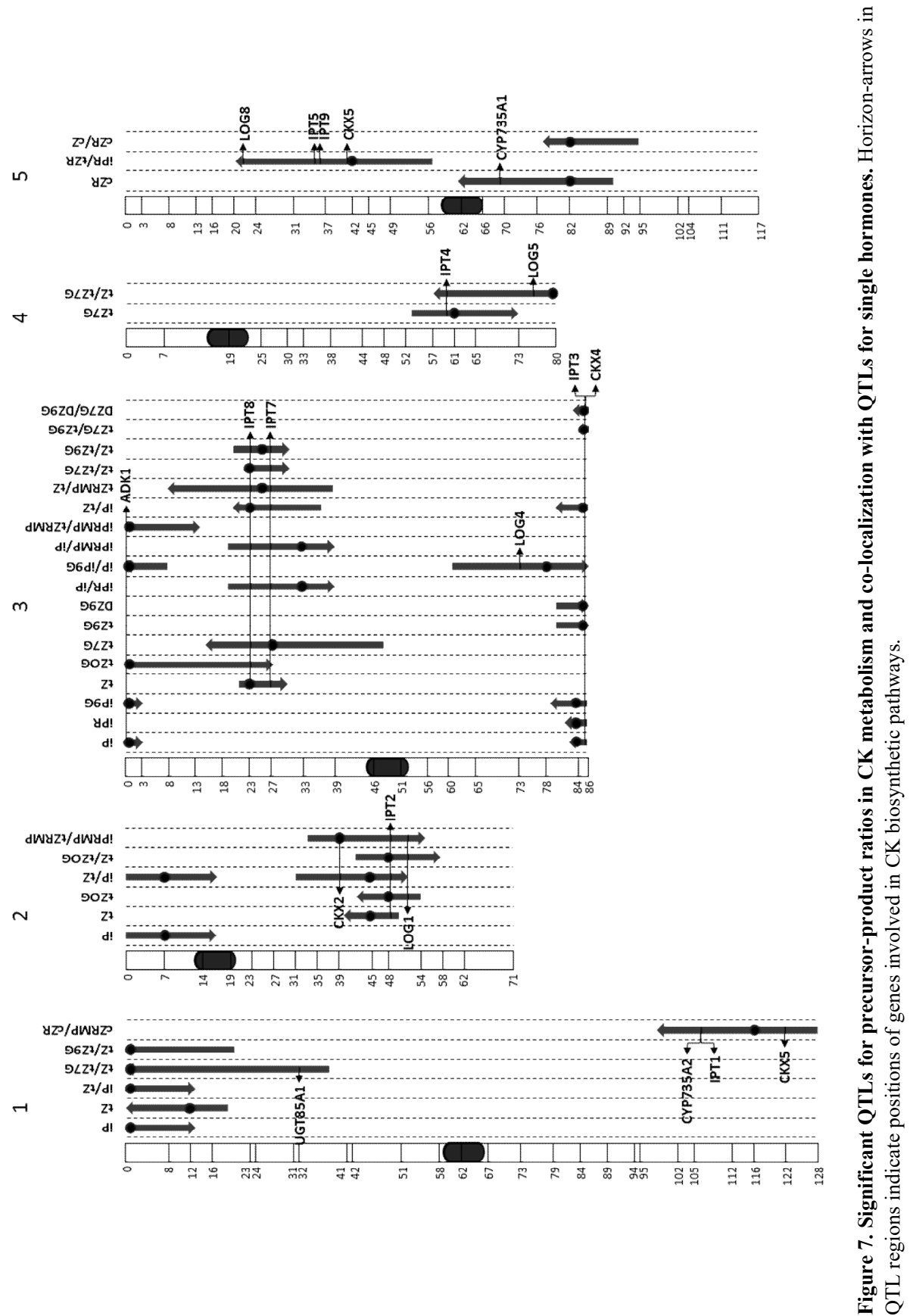


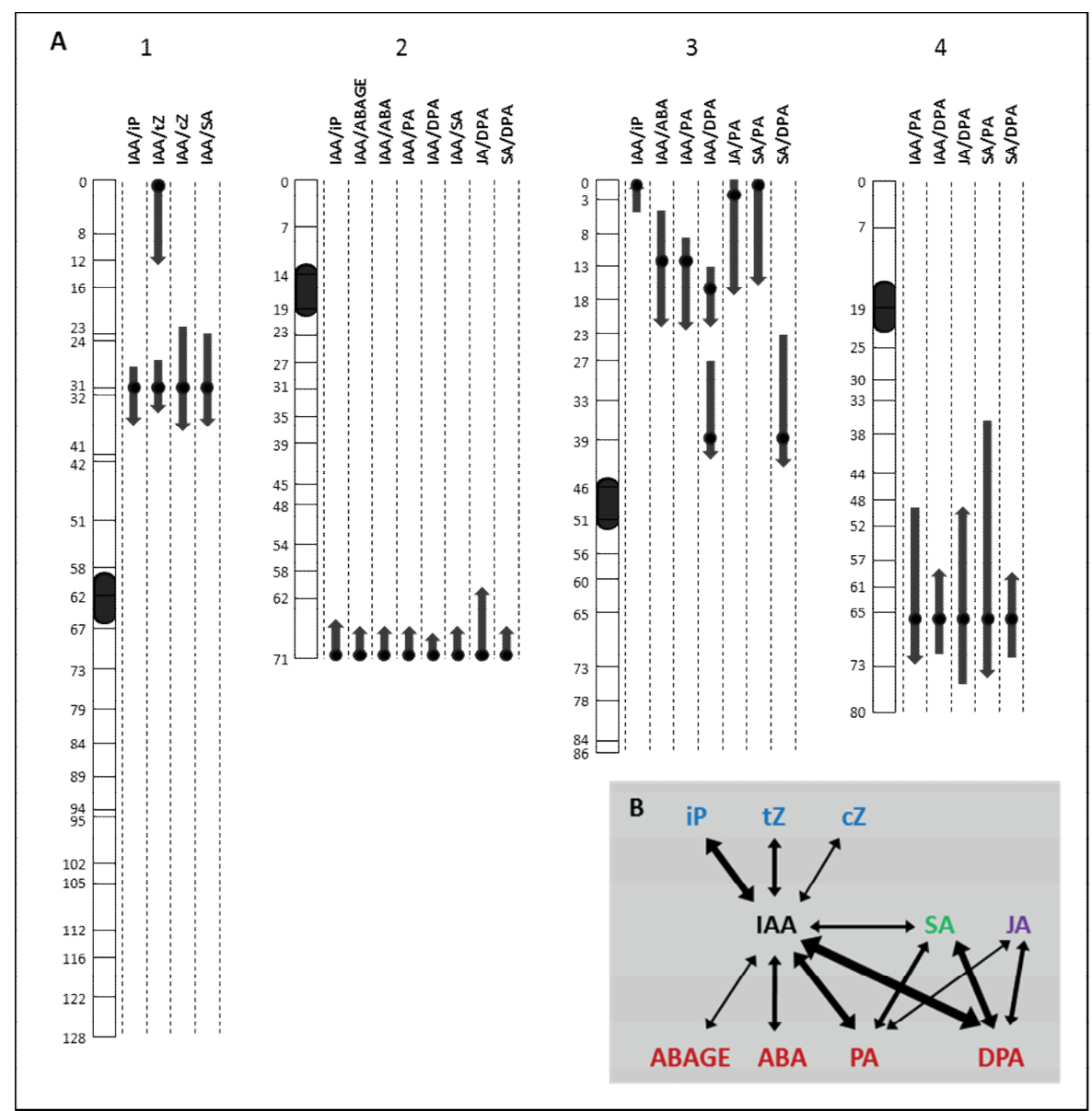

Figure 8. Significant QTLs for metabolite-metabolite ratios between different groups of hormone. (A), genetic locations of the QTLs. (B), schematic representation of numbers of significant QTLs. Thickness of the arrows represents numbers of metabolite-metabolite ratio QTLs found in pairs. The thinnest line is one QTL, e.g., IAA/SA and IAA/ABAGE. The thickest line (of IAA/DPA ratio QTL) represents 4 QTLs. IAA was the hormone showing the highest number of 'hormone-paired' QTLs, viz., 18 QTLs in total.

\section{QTLs for balances of CKs and SA between leaves and roots}

In order to find a QTL that might be involved in the hormone balances between leaves and roots, we obtained concentration ratios of hormones by combining hormone data (IAA, ABA, JA, SA, some CK metabolites) from leaves (as determined in the present paper) and roots as reported before (Lee et al., 2019). Figure 9 represents seven newly discovered QTL regions (diagonal stripes) for hormone ratios of CKs and SA between the two organs. For CK ratios, a new QTL was found for the leaftZRMP/root-tZRMP ratio at the lower arm of chromosome 1; five other ratio-QTLs co-localized with 
QTLs for single CK metabolites in either leaves or roots. A locus for the L/R-SA ratio was detected on the lower arm of chromosome 4, which positioned differently as compared to that for the SA level in roots found at the upper-arm of chromosome 1.

\section{1}

竎

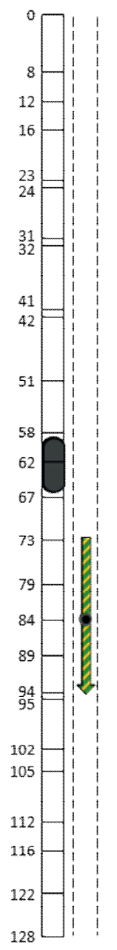

2
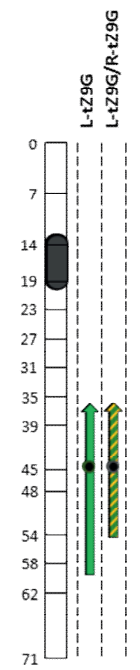

3

نั

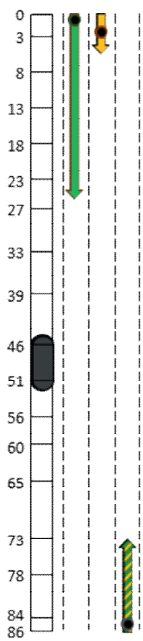

4
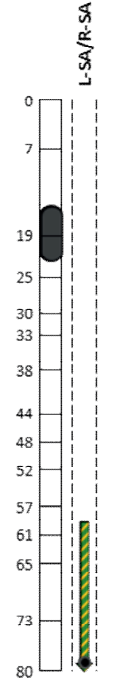

紊

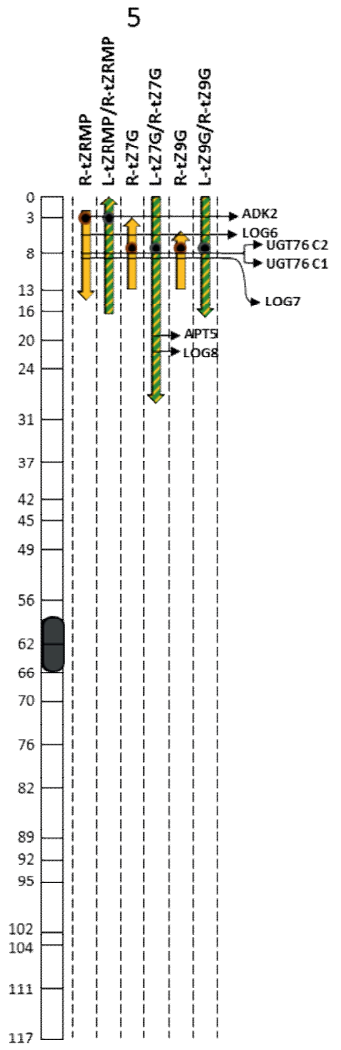

Figure 9. Hormone-ratio QTLs between two spatial organs, leaves and roots and their co-localizations with QTLs for single hormones. L- and R- indicate leaves and roots, respectively. Green arrows indicate single hormone QTL regions found in leaves, and yellow ones in roots. Diagonal arrows with alternate green and yellow stripes present hormone-ratios QTLs between two distant organs, leaves and roots. Horizon-arrows in QTL regions indicate positions of genes involved in CK biosynthetic pathways. See Table S7 to read exact QTL regions. 


\section{Discussion}

Fourteen hormone metabolites were commonly analyzed in leaves and roots of plants of the Arabidopsis RIL population (this paper and (Lee et al., 2019)). We compared levels of these hormones in leaves to those in roots observed in the two parental lines and the RILs (Table S2). Levels of CK monophosphates in leaves were several ten-folds lower than those in roots. Leaves also contained several fold lower levels of CK ribosides and IAA as compared to roots, but slightly higher levels of $\mathrm{CK}$ free bases (iP, tZ) and $\mathrm{ABA}$. Although there might be slight inaccuracies when comparing hormone quantities between two data sets obtained from different series of plantings and subsequent analyses, these data are in line with previous reports on differences in hormone levels between above-ground and below-ground organs of plants (Cai et al., 2016; Novak et al., 2008; Novak et al., 2012; Smehilova et al., 2016; Svacinova et al., 2012). Differences in hormone levels may be the results of: i), local synthesis of particular compounds; ii), organ-specific conversion to other metabolites, or breakdown; iii), hormone transport between organs. For example, auxin and activated forms of JA are spatiotemporally distributed in the root system, thus coordinating plant responses to environmental signals and developmental processes (Brunoud et al., 2012; Larrieu et al., 2016). QTLs for hormone levels are due to natural variation in the genes involved in these processes.

\section{Development-specific QTLs for hormone levels}

In the present study, development-specific QTLs were found in two sub-populations, viz., vegetative and flowering, as also found in roots of plants using the same RILs (Lee et al., 2017). The number of non-development-specific QTLs was relatively small compared with those that were developmentspecific, i.e., were observed either in the vegetative or the flowering stage. For IAA, all five detected QTLs displayed developmental changes in leaves and three of them were only detected in the subpopulation at the flowering stage (Figure 4). In some cases, significant QTLs observed in the whole population were composed of multiple development-specific QTLs, which were present either in the vegetative stage or in the flowering stage, e.g., co-localized QTLs at the lower arm of chromosome 2 for $\mathrm{tZ}$ and CK glucosides (Figure 5). Such complex, development-specific QTLs were frequently observed when LOD values of the QTL detected in the whole population were relatively high. It implies that, in several cases, QTLs for hormone levels consisted of more than one developmentspecific locus. Development-specific QTLs for linolenic acid and chlorophyll were reported in soybean and cotton, respectively (Han et al., 2011; Song and Zhang, 2010). Both growth-related traits and contents of primary and secondary metabolites are regulated by coordination of both life-long and development-specific QTLs (Bian et al., 2015; Li et al., 2015; Wurschum et al., 2014). In the quoted studies, whole populations were grown repetitively to study different developmental stages. An advantage of sampling when half of the lines in the whole population is vegetative, and the other half started to flower, is that development-specific QTLs can be determined in one experiment. This 
approach reduces time, labour and costs of instrumental analysis, and also excludes unwanted environmental variation due to varying culture conditions in consecutive experiments. A possible drawback might be that the power to detect QTLs might be different in sub-populations when differing too much in number of lines. Obviously this can be minimized when the number of genotypes in two sub-populations is nearly same, as described by Keurentjes et al. (2007a).

\section{The detection of hormone-QTLs depends on the tissue}

It is remarkable that multiple significant QTLs for levels of IAA and ABA were found in leaves, whereas, in our previous study, no loci for these hormones were observed in roots (Lee et al., 2019). This implies that the chance to detect QTLs is different according to organs and tissues, especially, in the case of hormones and/or other metabolites. This may be due to organ- or tissue-specific localization of biosynthesis of a particular hormone. Relatively larger quantities of IAA and ABA are synthesized in leaves, and in case of IAA it is basipetally transported to the root system and concentrated in the root tips (Ikeda et al., 2009; Merilo et al., 2015; Novak et al., 2012). CKs are synthesized in both roots and leaves, although some forms of CKs, for example, tZR, are transported from the root to the shoot via the xylem (Lacombe and Achard, 2016). These differences may have caused the detection of QTLs for CKs in both leaves and roots, but only in the leaves for IAA and ABAs.

It may also be that the possibility to detect hormone QTLs is influenced by differences in structural features of the analysed tissues between the two organs. Arabidopsis shoots consist mainly of leaves, which are repetitive anatomical structures, containing mesophyll cells and a vascular system spread out through the whole leaf. It means that the concentration of a hormone per square unit of leaf tissue is likely to be similar to that of other regions in the same leaf, and also probably not so much different from other leaves in the same rosette, although some variation is expected due to the age of leaves. This leads to a higher feasibility to detect a QTL for various groups of hormones. On the other hand, the root system is composed of different cell types horizontally and vertically, which may cause significant variation in hormone levels (Petersson et al., 2009). Such differential distribution of hormones in the root system is not taken into account when samples are prepared from whole roots, consequently leading to a lower power to detect QTLs. This might explain why no QTLs for IAA and ABA were found in the roots in our previous study (Lee et al., 2019).

\section{The ratio between two $\mathrm{CK} \mathrm{N}$-glucoside isomers may be a polygenic trait}

In the present study using the leaves of the Ler $\times$ Cvi RIL population, the concentration ratio between two CK conformational isomers (tZ7G : tZ9G) was $3.57: 1$, which was strikingly different from $0.97: 1$ in roots of the same RILs (Table S4). These results provide metabolic evidence that the conversion of $\mathrm{tZ7G}$ to $\mathrm{tZ9G}$ occurs biochemically. If the ratio resulted from a non-enzymatic process 
(tautomerism) as assumed in a previous study (Hou et al., 2004), the ratios should be the same or similar to each other irrespective of the difference of investigated organs. Therefore, we speculated that tZ9G is enzymatically converted from tZ7G. Moreover, the detection of significant QTLs for tZ9G and DZ9G on the bottom of chromosome 3 (Figure 5, Table S6) supports this possibility because these QTLs were only found without any QTL for tZ7G in the same region. Intriguingly, two QTLs for tZ7G/ tZ9G ratio in leaves and roots were separately located at chromosome 3 and 5, suggesting that the traits are polygenic and tissue-specific (Table 2, Lee et al. (2019)).

Table 2. Significant QTLs for metabolites ratios between $\mathrm{CK}-\mathrm{N}$-glucosides in leaves and roots.

\begin{tabular}{|c|c|c|c|c|c|c|c|c|}
\hline Organ & Ratio trait & $\begin{array}{c}\text { Chromo- } \\
\text { some }\end{array}$ & $\begin{array}{c}\text { cM } \\
\text { interval }\end{array}$ & $\begin{array}{c}\text { LOD } \\
\text { (MQM) }\end{array}$ & $\begin{array}{l}\text { Locus } \\
\text { region }\end{array}$ & $\begin{array}{l}\text { Nearest } \\
\text { marker }\end{array}$ & $\begin{array}{c}\text { Explained } \\
\text { Variance (\%) }\end{array}$ & $\begin{array}{c}\text { Additive } \\
\text { effect }\end{array}$ \\
\hline \multirow{2}{*}{ leaves } & tZ7G/tZ9G & 3 & $85 \sim 85.8$ & 25.8 & BH.109L-Col HH.90L-Col & HH.90L-Col & 54.9 & + \\
\hline & DZ7G/DZ9G & 3 & $82.4 \sim 85.8$ & 14.7 & HH.171C-Col/173L $\sim H H .90 L-C o l$ & HH.9OL-Col & 36.5 & + \\
\hline roots & tZ7G/tZ9G & 5 & $62.5 \sim 81.5$ & 3.3 & BH.96L-Col AD.75C-Col & CD.116L & 10 & - \\
\hline
\end{tabular}

In Arabidopsis, a few isomerases have been reported, for example, those of chorismate phosphoglycerate (Konishi et al., 2007; Romero et al., 1995; Zhao and Assmann, 2011). Hence, we suppose that the determination of isomeric compounds in a quantitative genetics study and the use of ratio data may lead us to find new genetic elements involved in biosynthetic pathways. In addition, in the present study precursor-product ratio QTLs co-localized with QTLs for single compounds of either substrates or products in pair, suggesting that some of the QTLs for the levels of single compounds also regulate the balance between metabolites in a given biosynthetic pathway.

\section{QTLs for hormone balance between different classes of hormone}

Plant hormones, e.g., auxin and CK interact with each other to modulate growth and development of plants (Schaller et al., 2015). CK plays a role as a positive regulator of auxin biosynthesis, resulting in a homeostatic feedback mechanism in roots and shoots (Jones et al., 2010). Within five different groups of hormone and their metabolites, the number of hormone-hormone ratio QTLs was different according to sorts of hormone-hormone pairs. Figure 8B depicts QTLs for the chemical pairs (lines) and their numbers (thickness of lines). Auxin had a central position in terms of the number of QTLs for specific hormone-hormone ratios and the number of interactions with other hormones. Except for the matching with auxin, no other QTL for ratios of CK metabolites-to-other hormones (ABAs, SA and JA) were observed. The detection of a multitude of QTLs for ratios of auxin-to-other hormones is consistent with the physiologically central role of auxin as interconnecting mediator in plant development and growth (Jaillais and Chory, 2010). This is also in the line with accumulated findings 
that a large proportion of auxin hormone crosstalk is associated with effects on biosynthesis or metabolism of most other hormones (Nemhauser et al., 2006).

\section{Gene clusters related to CK biosynthetic pathways on CK QTLs}

It is noteworthy that many genes related to CK metabolism locate in significant QTL regions for single hormones and metabolite-ratios (Figure 6B). Regarding CK biosynthetic pathways (Figure 3), these genes include IPTs responsible for the first step of isoprenoid CK biosynthesis and up to CKXs for direct inactivation of biologically active CKs. The frequent observation of co-localized QTLs for metabolite-ratios (relations in precursor-product) and single hormones suggests that some of the genetic elements underlying QTLs are likely to be directly involved in CK biosynthetic pathways. This idea can be partially supported by a previous finding that IPTs play a role in the rate-limiting step for iP and $\mathrm{tZ}$ in CK biosynthesis (Miyawaki et al., 2006).

Not all QTLs for CK contain such genes directly involved in the biosynthetic pathways. For example, QTL regions at the top of chromosome 1 and 2 did not hold any known genes for CK metabolism. These QTLs may rather include transcription factors involved in CK signalling pathway because it has been known that phosphorylation and signal transduction are required for the function in negative feedback regulation, resulting in CK metabolic balances (Werner and Schmulling, 2009). In cases of ratio QTLs between different groups of hormone, underlying genes may be more likely involved in signalling components via receptors because it is hard to conceive that a specific biosynthetic enzyme in a hormone metabolic pathway reacts to structurally unrelated metabolites $(\mathrm{Li}$ et al., 2014). A QTL for ratios between different groups of hormones can be an interesting target region for further studies in breeding programs. This is because changes in the ratios of hormones have a significant role in determining stress tolerances (Wilkinson et al., 2012). For instance, the sensitivity of stress responses is dependent on the ratio between ethylene and ABA, more so than the single hormones alone (Acharya and Assmann, 2009; Wilkinson and Davies, 2010).

\section{Genetically regulated hormone balances between leaves and roots}

In Arabidopsis, several transporters for hormones have been reported such as PINs for polar auxin transport (Friml et al., 2003; Sabatini et al., 1999) and ATP-binding cassette transporters (ABCGs) for long distance translocation of CK (Ko et al., 2014; Zhang et al., 2014). In the present study, the detection of QTLs for shoot-root ratios of hormone levels suggests that the underlying regions contain genes to be responsible for hormone balances, especially, at two loci for CK ratios (tZRMP in chromosome 1 and tZOG in chromosome 2) and a locus for SA on chromosome 4, which did no colocalize with the QTL for the compounds that determine the ratio in either the leaves or the roots. All of these five regions did not include any known transport-related genes for hormones (Figure 7). Remarkably, in our study no QTLs for the balance of auxin and ABA were found, but only QTLs for 
balances of tZRMP, tZ7G and tZ9G, although these compounds are not known as transport forms of CK (Osugi et al., 2017). These results suggest that the chance to detect a QTL for controlling hormone balances between leaves and roots is relatively low and the hormone levels might be mainly determined by metabolic fluxes in local tissues, rather than translocation through the vascular system.

\section{Conclusion}

We have found a multitude of QTLs determining the levels of several different hormones and their metabolites in leaves of Arabidopsis. In two sub-populations (vegetative and flowering), development-specific QTLs were found. In many cases, hormone ratio QTLs co-localized with the QTLs for either one of the single compounds in these pairs, suggesting that relevant loci also affect the synthesis and degradation of intermediates in hormone metabolism. The detection of QTLs for ratios between structurally unrelated hormones (e.g., auxin : ABA) opens up the way to unravel genetic elements underlying hormonal interactions in the regulation of plant development and stress responses.

\section{Acknowledgements}

The authors thank Dr. Leonie Bentsink for providing seeds of the Ler $\times$ Cvi RIL populations. 


\section{[Supplementary Information]}

Table S1. Summary of multiple reaction monitor (MRM) transitions used for hormone quantification in UPLC-ESI-TQ MS.

\begin{tabular}{|c|c|c|c|c|c|c|c|c|c|c|c|}
\hline Analytes & $\begin{array}{c}\text { UPLC } \\
\text { Gradient }\end{array}$ & $\mathrm{RT}$ & $\begin{array}{c}\text { ESI } \\
\text { mode }\end{array}$ & Transition & $\begin{array}{c}\text { Cone } \\
\text { voltage }\end{array}$ & $\begin{array}{c}\text { Collision } \\
\text { energy }\end{array}$ & $\begin{array}{l}\text { Internal } \\
\text { standard }\end{array}$ & RT & Transition & $\begin{array}{c}\text { Cone } \\
\text { voltage }\end{array}$ & $\begin{array}{c}\text { Collision } \\
\text { energy }\end{array}$ \\
\hline$\overline{\text { iPRMP }}$ & 1 & 4.56 & + & $416.2>204.1$ & 34 & 22 & {$\left[{ }^{2} \mathrm{H}_{5}\right]$ tZRMP } & 1.85 & $437.2>225.1$ & 34 & 22 \\
\hline $\mathrm{iPR}$ & 1 & 7.60 & + & $336.1>204.1$ & 28 & 18 & {$\left[{ }^{2} \mathrm{H}_{6}\right] \mathrm{iPR}$} & 7.55 & $342.1>210.1$ & 28 & 18 \\
\hline iP & 1 & 6.15 & + & $204.1>136.1$ & 20 & 18 & {$\left[{ }^{2} \mathrm{H}_{6}\right] \mathrm{iP}$} & 6.11 & $210.1>137.1$ & 20 & 18 \\
\hline iP9G & 1 & 6.52 & + & $366.2>204.1$ & 30 & 20 & {$\left[{ }^{2} \mathrm{H}_{6}\right]$ iP9G } & 6.46 & $372.2>210.1$ & 30 & 20 \\
\hline tZRMP & 1 & 1.86 & + & $432.2>220.1$ & 34 & 22 & {$\left[{ }^{2} \mathrm{H}_{5}\right]$ tZRMP } & 1.85 & $437.2>225.1$ & 34 & 22 \\
\hline tZR & 1 & 5.04 & + & $352.1>136.1$ & 28 & 18 & {$\left[{ }^{2} \mathrm{H}_{5}\right]$ tZR } & 5.01 & $357.1>136.9$ & 28 & 18 \\
\hline $\mathrm{tz}$ & 1 & 3.78 & + & $220.1>136.1$ & 20 & 18 & {$\left[{ }^{2} \mathrm{H}_{5}\right] \mathrm{tZ}$} & 3.75 & $225.1>136.9$ & 20 & 18 \\
\hline tZOG & 1 & 3.84 & + & $382.1>220.1$ & 30 & 20 & {$\left[{ }^{2} \mathrm{H}_{5}\right]$ tZ9G } & 4.02 & $387.2>225.2$ & 30 & 20 \\
\hline $\mathrm{tZ7G}$ & 1 & 3.86 & + & $382.1>220.1$ & 30 & 20 & {$\left[{ }^{2} \mathrm{H}_{5}\right] \mathrm{tZ9G}$} & 4.02 & $387.2>225.2$ & 30 & 20 \\
\hline tZ9G & 1 & 4.04 & + & $382.1>220.1$ & 30 & 20 & {$\left[{ }^{2} \mathrm{H}_{5}\right] \mathrm{tZ9G}$} & 4.02 & $387.2>225.2$ & 30 & 20 \\
\hline $\mathrm{DZ}$ & 1 & 3.95 & + & $222.1>136.1$ & 20 & 18 & {$\left[{ }^{2} \mathrm{H}_{3}\right] \mathrm{DZ}$} & 3.91 & $225.1>136.1$ & 20 & 18 \\
\hline DZR & 1 & 5.10 & + & $354.1>136.1$ & 28 & 22 & {$\left[{ }^{2} \mathrm{H}_{3}\right] \mathrm{DZR}$} & 5.06 & $357.2>149.1$ & 28 & 22 \\
\hline DZ7G & 1 & 2.97 & + & $384.2>222.1$ & 30 & 20 & {$\left[{ }^{2} \mathrm{H}_{5}\right]$ tZ9G } & 4.02 & $387.2>225.2$ & 30 & 20 \\
\hline DZ9G & 1 & 3.36 & + & $384.2>222.1$ & 30 & 20 & {$\left[{ }^{2} \mathrm{H}_{5}\right] \mathrm{tZ9G}$} & 4.02 & $387.2>225.2$ & 30 & 20 \\
\hline cZRMP & 1 & 1.92 & + & $432.2>220.1$ & 34 & 22 & {$\left[{ }^{2} \mathrm{H}_{5}\right]$ tZRMP } & 1.85 & $437.2>225.1$ & 34 & 22 \\
\hline cZR & 1 & 4.49 & + & $352.2>220.1$ & 28 & 22 & {$\left[{ }^{2} \mathrm{H}_{5}\right] \mathrm{tZR}$} & 5.01 & $357.1>136.9$ & 28 & 18 \\
\hline$c Z$ & 1 & 4.42 & + & $220.1>136.1$ & 20 & 18 & {$\left[{ }^{2} \mathrm{H}_{6}\right] \mathrm{tZ}$} & 3.75 & $225.1>136.9$ & 20 & 18 \\
\hline IAA & 2 & 4.23 & + & $176.3>130.2$ & 25 & 15 & {$\left[{ }^{13} \mathrm{C}_{6}\right]$ IAA } & 4.22 & $182.1>135.9$ & 25 & 15 \\
\hline ABA & 2 & 5.03 & - & $263.1>153.1$ & 25 & 10 & {$\left[{ }^{2} \mathrm{H}_{6}\right] \mathrm{ABA}$} & 5.01 & $269.1>159.1$ & 25 & 10 \\
\hline ABAGE & 2 & 3.53 & - & $425.0>262.7$ & 30 & 15 & {$\left[{ }^{2} \mathrm{H}_{5}\right]$ ABAGE } & 3.50 & $430.0>267.7$ & 30 & 15 \\
\hline PA & 2 & 3.71 & - & $279.0>138.9$ & 30 & 15 & {$\left[{ }^{2} \mathrm{H}_{3}\right] \mathrm{PA}$} & 3.69 & $282.0>142.2$ & 30 & 15 \\
\hline DPA & 2 & 1.78 & - & $281.0>171.2$ & 30 & 20 & {$\left[{ }^{2} \mathrm{H}_{3}\right]$ DPA } & 1.76 & $284.0>174.0$ & 30 & 20 \\
\hline JA & 2 & 5.82 & - & $209.2>58.8$ & 30 & 15 & {$\left[{ }^{2} \mathrm{H}_{6}\right] \mathrm{JA}$} & 5.85 & $215.0>58.8$ & 30 & 15 \\
\hline SA & 2 & 5.22 & - & $137.06>92.8$ & 25 & 15 & {$\left[{ }^{2} \mathrm{H}_{4}\right] \mathrm{SA}$} & 5.17 & $141.1>96.8$ & 25 & 15 \\
\hline
\end{tabular}


Table S2. Quantitative fold differences of hormone metabolites between roots and leaves in parental lines and the RILs. Numerical values were obtained from averages of hormone levels ( $\left.\mathrm{pg} \mathrm{mg}^{-1} \mathrm{dw}\right)$ in root divided by hormone levels in leaves.

\begin{tabular}{lccc}
\hline & Ler & Cvi & RILs \\
\hline iPRMP & 26.3 & 15.3 & 12.7 \\
iP & 0.4 & 0.3 & 0.2 \\
tZRMP & 102.1 & 55.5 & 58.5 \\
tZR & 9.8 & 4.1 & 5.7 \\
tZ & 0.7 & 1.6 & 0.5 \\
tZOG & 4.1 & 8.9 & 4.1 \\
tZ7G & 0.6 & 0.4 & 0.5 \\
tZ9G & 3.0 & 1.2 & 1.8 \\
cZR & 14.2 & 11.0 & 7.1 \\
cZ & 1.6 & 1.3 & 1.1 \\
IAA & 7.8 & 20.0 & 18.5 \\
ABA & 0.3 & 0.3 & 0.4 \\
JA & 21.4 & 26.9 & 11.7 \\
SA & 134.2 & 168.0 & 294.3 \\
\hline
\end{tabular}




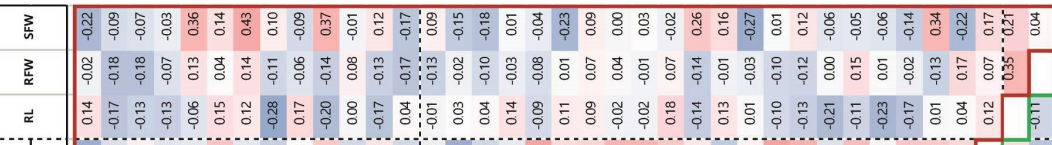

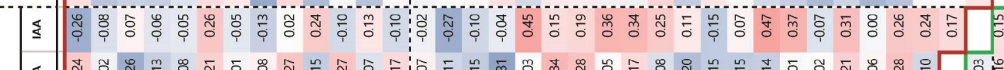

๘

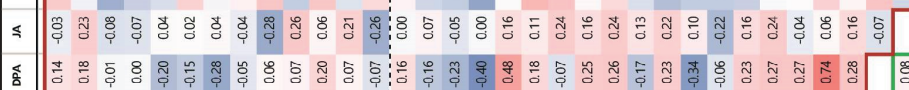

䢓

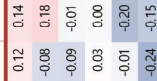

要

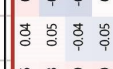

崖

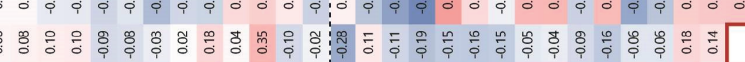

范

ป

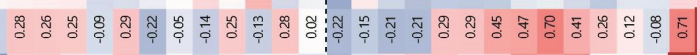

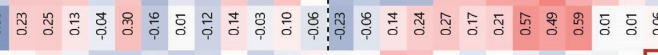

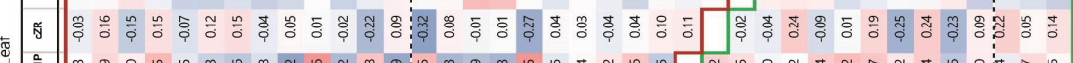

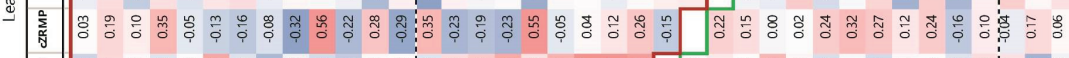

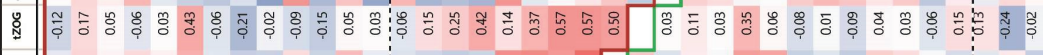

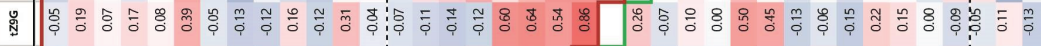

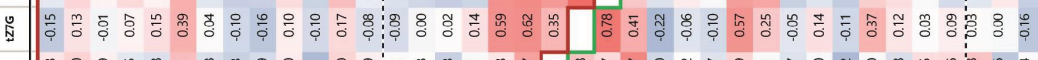

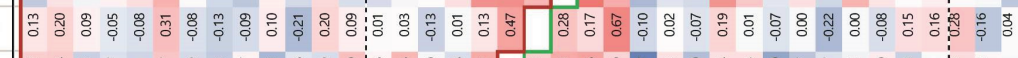

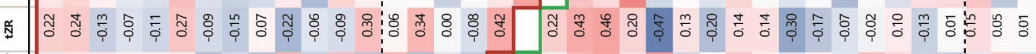

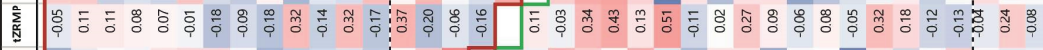

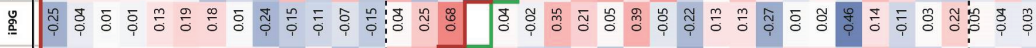

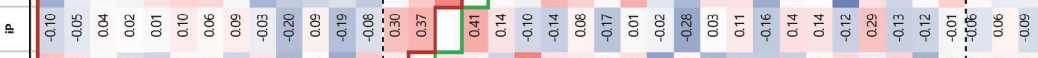

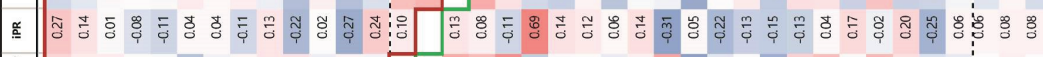

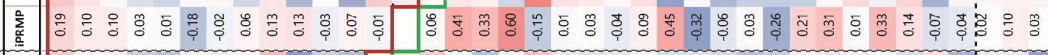

ธ

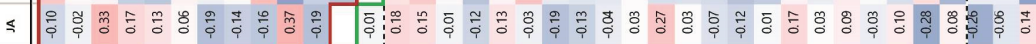

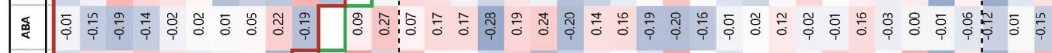

新

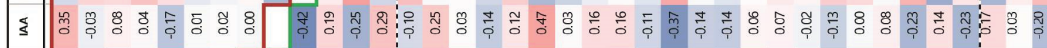

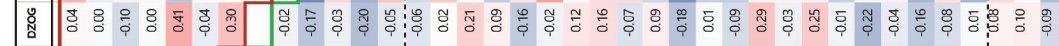

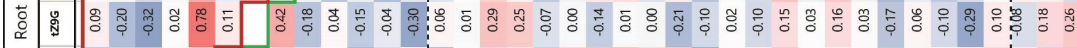

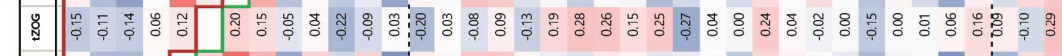

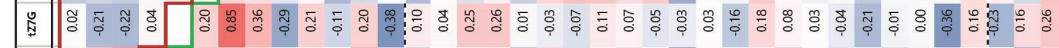

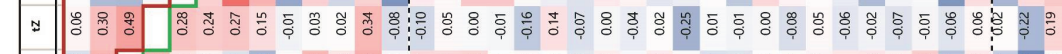

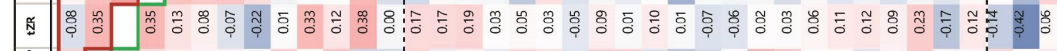

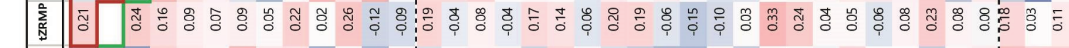

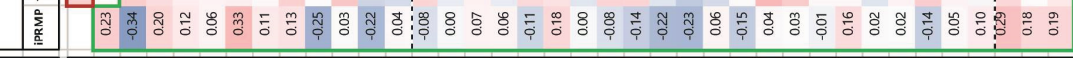

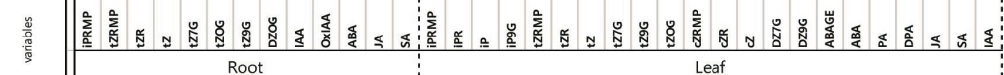

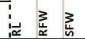


Table S4. tZ7G/tZ9G ratios observed in roots and leaves.

\begin{tabular}{lllll}
\hline Organs & Ler & Cvi & RILs & Col-0 \\
\hline roots & 0.91 & 1.13 & 0.97 & 1.95 \\
leaves & 4.83 & 3.32 & 3.57 & 3.50 \\
\hline
\end{tabular}

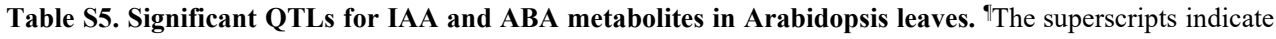
flowering-specific QTLs that were detected from only the flowered sub-population. ${ }^{\S}$ The superscripts indicate vegetative-specific QTLs that were detected from only the vegetative sub-population.

\begin{tabular}{|c|c|c|c|c|c|c|c|}
\hline Trait & $\begin{array}{c}\text { Chromo- } \\
\text { some }\end{array}$ & $\begin{array}{c}\text { cM } \\
\text { interval }\end{array}$ & $\begin{array}{c}\text { LOD } \\
\text { (MQM) }\end{array}$ & $\begin{array}{l}\text { Locus } \\
\text { region }\end{array}$ & $\begin{array}{l}\text { Nearest } \\
\text { marker }\end{array}$ & $\begin{array}{c}\text { Explained } \\
\text { Variance (\%) }\end{array}$ & $\begin{array}{c}\text { Additive } \\
\text { effect }\end{array}$ \\
\hline \multirow{5}{*}{ IAA } & 1 & $25.3 \sim 36.7$ & 7.4 & GD.86L AD.106L-Col & CC.98L-Col/101C & 16 & - \\
\hline & \multirow{3}{*}{2} & $36.4 \sim 47.6$ ๆ & 2.5 & BF.221L Erecta/GPA & FD.150C & 12.5 & + \\
\hline & & $49.2 \sim 62.3$ ๆ & 2.7 & Erecta/GPA DF.140C & GD.298C & 14.1 & + \\
\hline & & $65.3^{\sim 70.8}$ & 5.4 & DF.140C EC.235L-Col/247C & EC.235L-Col/247C & 11.3 & + \\
\hline & 3 & $0 \sim 3.8$ ๆ & 2.5 & DF.77C EG.75L & DF.77C & 13.2 & + \\
\hline \multirow{2}{*}{ ABAGE } & \multirow{2}{*}{1} & $13.1 \sim 22.5^{\S}$ & 2.7 & HH.335C-Col/PhyA EC.66C & EC. $480 \mathrm{C}$ & 14.9 & - \\
\hline & & $85.4 \sim 104.1$ ๆ & 4.0 & EC.88C GH.157L-Col & CH.215L & 21.3 & + \\
\hline \multirow{5}{*}{$\mathrm{ABA}$} & 1 & $13.1 \sim 39.7$ & 4.0 & HH.335C-Col/PhyA AD.106L-Col & GD.86L & 7.5 & - \\
\hline & \multirow{2}{*}{3} & $0 \sim 17.1$ & 5.9 & DF.77C BF.270L-Col/271C & GB.120C-Col/GAPC & 11.5 & + \\
\hline & & $72.9 \sim 85.8$ & 2.7 & AD.182C HH.9OL-Col & HH.9OL-Col & 4.9 & + \\
\hline & \multirow{2}{*}{5} & $32.1 \sim 52.6$ & 3.2 & DF.184L-Col BH.96L-Col & GH.121L-Col & 6.0 & - \\
\hline & & $94.9 \sim 117$ & 3.2 & GB.102L-Col/105C HH.122C/120L & DF.119L & 5.9 & + \\
\hline \multirow[t]{3}{*}{ PA } & 3 & $0 \sim 15.1$ & 4.6 & DF.77C BF.270L-Col/271C & DF.77C & 12.5 & + \\
\hline & 2 & $67.3 \sim 70.8$ & 11.1 & DF.140C EC.235L-Col/247C & EC. $235 \mathrm{~L}-\mathrm{Col} / 247 \mathrm{C}$ & 16.9 & - \\
\hline & & $0 \sim 8.8$ ๆ & 3.3 & DF.77C EG.75L & DF.77C & 3.3 & + \\
\hline \multirow[t]{3}{*}{ DPA } & 3 & $31.4 \sim 45.5$ & 3.4 & EC. $83 \mathrm{C} / 84 \mathrm{~L} \sim \mathrm{BH} .225 \mathrm{C}-\mathrm{Col}$ & GB.210L & 4.5 & + \\
\hline & & $75.9 \sim 85.8^{\S}$ & 2.5 & AD.182C HH.90L-Col & HH.90L-Col & 6.2 & + \\
\hline & 4 & $59 \sim 68$ & 13.9 & CH.70L/71C-Col BH.342C & HH.159C-Col & 21.8 & - \\
\hline
\end{tabular}


Table S6. Significant QTLs of CKs in rosette leaves. ` The superscripts indicate flowering-specific QTLs that were detected from only the flowered sub-population. ${ }^{\S}$ The superscripts indicate vegetative-specific QTLs that were detected from only the vegetative sub-population.

\begin{tabular}{|c|c|c|c|c|c|c|c|}
\hline Trait & $\begin{array}{c}\text { Chromo- } \\
\text { some }\end{array}$ & $\begin{array}{c}\text { cM } \\
\text { interval }\end{array}$ & $\begin{array}{c}\text { LOD } \\
\text { (MQM) }\end{array}$ & $\begin{array}{l}\text { Locus } \\
\text { region }\end{array}$ & $\begin{array}{l}\text { Nearest } \\
\text { marker }\end{array}$ & $\begin{array}{c}\text { Explained } \\
\text { Variance (\%) }\end{array}$ & $\begin{array}{c}\text { Additive } \\
\text { effect }\end{array}$ \\
\hline iPR & 3 & $81.4 \sim 85.8$ & 2.9 & HH.171C-Col/173L HH.90L-Col & HH.90L-Col & 8.2 & + \\
\hline \multirow{4}{*}{ iP } & 1 & $0 \sim 13.1$ & 3.6 & PVV4 HH,335C-Col/PhyA & PVV4 & 7.3 & - \\
\hline & 2 & $0 \sim 16.9$ & 3.6 & AD.156C FD.81L & GH.580L & 7.1 & - \\
\hline & \multirow{2}{*}{3} & $0 \sim 2$ & 3.7 & DF.77C GB.120C-Col/GAPC & DF.77C & 7.5 & - \\
\hline & & $82.4 \sim 85.8$ & 6.5 & HH.171C-Col/173L HH.90L-Col & HH.90L-Col & 13.6 & + \\
\hline \multirow{4}{*}{ iP9G } & \multirow{2}{*}{3} & $0 \sim 2$ & 15.7 & DF.77C GB.120C-Col/GAPC & DF.77C & 30 & - \\
\hline & & $79.4 \sim 85.8$ & 8.7 & HH.171C-Col/173L HH.90L-Col & HH.9OL-Col & 14.7 & + \\
\hline & \multirow{2}{*}{5} & $0 \sim 11.6$ & 5.5 & FD.207L EC.198L-Col & $\mathrm{CH} .690 \mathrm{C}$ & 8.7 & + \\
\hline & & $107.5^{\sim 115}$ ๆ & 4.1 & BF.168L-Col HH.122C/120L & DF.119L & 11.6 & - \\
\hline tZRMP & 4 & $50.1 \sim 73$ & 2.6 & CD.329C-Col BH.342C/DHS1 & CH.70L/71C-Col & 6.8 & - \\
\hline \multirow{4}{*}{ tZR } & 1 & $77.4 \sim 100.3$ ๆ & 2.5 & CH.200C BF.116C & GD.160C & 9.7 & - \\
\hline & \multirow{2}{*}{4} & $0 \sim 18.3^{\S}$ & 2.8 & ANL2 CH.169C & GH.250C/GA1 & 16.4 & - \\
\hline & & $51.1 \sim 60$ ๆ & 2.7 & CD.329C-Col HH.159C-Col & CH.70L/71C-Col & 10.7 & - \\
\hline & 5 & $9.6 \sim 34.1$ ๆ & 4.0 & BH.144L GH.117C & BH.107L-Col & 16.8 & - \\
\hline \multirow{7}{*}{$\mathrm{tZ}$} & 1 & $0 \sim 20.3$ & 4.8 & PVV4 EC. $66 \mathrm{C}$ & HH.335C-Col/PhyA & 9.8 & + \\
\hline & 2 & $40.4 \sim 50.2$ & 8.6 & FD.85C GD.298C & FD.150C & 18.8 & + \\
\hline & 3 & $21.1 \sim 30.4$ & 6.8 & BF.270L-Col/271C AD.92L & GH.390L & 14.3 & - \\
\hline & \multirow{2}{*}{4} & $0^{\sim} 18.3^{\S}$ & 2.6 & ANL2 CH.169C & GH.250C/GA1 & 7.5 & - \\
\hline & & $20.2^{\sim} 37.1^{\S}$ & 2.6 & CH.169C CD.84C-Col & $\mathrm{BH} .92 \mathrm{~L}-\mathrm{Col}$ & 7.6 & - \\
\hline & \multirow{2}{*}{5} & $91.7^{\sim} 102.7^{\S}$ & 2.5 & HH.445L-Col HH.143C & GB.102L-Col & 7.2 & + \\
\hline & & $104.5^{\sim} 117^{\S}$ & 3.0 & BF.168L-Col HH.122C/120L & DF.119L & 8.6 & + \\
\hline \multirow{3}{*}{ tZOG } & 1 & $4 \sim 20.3$ & 6.1 & PVV4 EC.66C & EC. $480 \mathrm{C}$ & 12.3 & + \\
\hline & 2 & $43.4 \sim 51.2$ & 12.7 & FD.85C GD.298C & Erecta/GPA1 & 29.5 & + \\
\hline & 3 & $0 \sim 26.1$ & 3.0 & DF.77C EC.83C/84L & DF.77C & 5.5 & - \\
\hline \multirow{5}{*}{$\mathrm{tZ7G}$} & 2 & $39.4 \sim 63.3$ & 5.7 & FD.85C DF.140C & GD.298C & 11.4 & + \\
\hline & 3 & $28.4^{\sim} 72.3$ & 2.8 & EC.83C/84L AD.182C & $\mathrm{HH} .440 \mathrm{~L}$ & 5.4 & + \\
\hline & 4 & $55.6 \sim 74$ & 6.3 & FD.167L-Col BH.342C/DHS1 & HH.159C-Col & 12.6 & - \\
\hline & \multirow{2}{*}{5} & $19.5 \sim 54.6$ & 2.7 & BH.107L-Col BH.96L-Col & GH.117C & 5.2 & - \\
\hline & & $79.5^{\sim} 105.5$ & 4.0 & DFR BF.168L-Col & $\mathrm{HH} .445 \mathrm{~L}-\mathrm{Col}$ & 7.6 & - \\
\hline \multirow{5}{*}{ tZ9G } & 2 & $36.4 \sim 60.2$ & 4.9 & BF.221L BH.120L-Col & FD.150C & 10.9 & + \\
\hline & 3 & $80.4 \sim 85.8$ & 4.3 & HH.171C-Col/173L HH.90L-Col & HH.9OL-Col & 8.4 & - \\
\hline & 4 & $54.6 \sim 71$ & 5.3 & FD.167L-Col BH.342C/DHS1 & HH.159C-Col & 11.7 & - \\
\hline & \multirow{2}{*}{5} & $21.5 \sim 54.6$ & 2.7 & BH.107L-Col BH.96L-Col & GH.117C & 7.3 & - \\
\hline & & $92 \sim 108.5$ & 2.8 & CC.262C DF.119L & HH.143C & 7.3 & - \\
\hline \multirow{4}{*}{ DZ7G } & 1 & $90.7^{2} 108$ ๆ & 3.5 & GD.160C CC.318C & BF.116C & 9.6 & + \\
\hline & 2 & $38.4 \sim 47.6$ & 8.5 & BF.221L Erecta/GPA1 & FD.85C & 16.8 & + \\
\hline & 4 & $52.6 \sim 78$ & 6.0 & FD.167L-Col BH.342C/DHS1 & HH.159C-Col & 11.3 & - \\
\hline & 5 & $103.5^{\sim 117}$ ๆ & 3.7 & BF.168L-Col HH.122C/120L & DF.119L & 9.9 & - \\
\hline \multirow{5}{*}{ DZ9G } & \multirow{2}{*}{2} & $29.1 \sim 45.6$ & 2.5 & FD.222L-Col Erecta/GPA1 & FD.85C & 4.1 & + \\
\hline & & $49.2 \sim 56.6$ & 6.8 & Erecta/GPA1 BH.120L-Col & GD.298C & 6.8 & + \\
\hline & 3 & $80.4 \sim 85.8$ & 12.5 & HH.171C-Col/173L HH.90L-Col & $\mathrm{HH} .90 \mathrm{~L}-\mathrm{Col}$ & 23.8 & - \\
\hline & 4 & $54.6 \sim 69$ & 7.4 & FD.167L-Col BH.342C/DHS1 & HH.159C-Col & 12.8 & - \\
\hline & 5 & $25.3 \sim 40.8$ ๆ & 3.0 & AD.114C-Col GH.121L-Col & DF.184L-Col & 6.9 & - \\
\hline \multirow{2}{*}{ cZR } & 3 & $0 \sim 8.8$ & 4.7 & DF.77C FD.111L-Col/136C & DF.77C & 11.3 & - \\
\hline & 5 & $64.5^{\sim 90.7}$ & 3.8 & CD.179L CC.262C & AD.75C-Col & 9.9 & + \\
\hline$c Z$ & 4 & $4 \sim 23.2$ & 2.9 & ANL2 EC.306L & GH.250C/GA1 & 8.4 & + \\
\hline
\end{tabular}


Table S7. Significant QTLs of hormone ratios between two organs, rosette leaves and roots.

\begin{tabular}{cccccccc}
\hline Trait & $\begin{array}{c}\text { Chromo- } \\
\text { some }\end{array}$ & $\begin{array}{c}\text { cM } \\
\text { interval }\end{array}$ & $\begin{array}{c}\text { LOD } \\
\text { (MQM) }\end{array}$ & $\begin{array}{c}\text { Locus } \\
\text { region }\end{array}$ & $\begin{array}{c}\text { Nearest } \\
\text { marker }\end{array}$ & $\begin{array}{c}\text { Explained } \\
\text { Variance (\%) }\end{array}$ & $\begin{array}{c}\text { Additive } \\
\text { effect }\end{array}$ \\
\hline \multirow{2}{*}{ tZRMP } & 1 & $72 \sim 94.4$ & 4.1 & BF.206L-Col HH.375L & EC.88C & 12 & - \\
\cline { 2 - 8 } & 5 & $0 \sim 16.8$ & 3.2 & FD.207L BH.107-Col & CH.690C & 9.3 & + \\
\hline \multirow{2}{*}{ tZOG } & 1 & $0 \sim 19.3$ & 4.0 & PVV4 EC.66C & HH.335-Col/PhyA & 9.7 & + \\
\cline { 2 - 9 } & 4 & $72.3 \sim 85.8$ & 2.9 & AD.182C HH.90L-Col & HH.90L-Col & 7 & + \\
\hline tZ7G & 5 & 0 28.3 & 3.2 & FD.207L AD.114C-Col & BH.144L & 9.4 & - \\
\hline \multirow{2}{*}{ tZ9G } & 2 & $35.4 \sim 54.6$ & 3.2 & BF.221L GD.298C & FD.150C & 8.9 & + \\
\cline { 2 - 8 } & 5 & $2 \sim 16.8$ & 4.8 & FD.207L BH.107L-Col & BH.144L & 13.6 & - \\
\hline SA & 4 & $59 \sim 80.2$ & 3.1 & HH.159C-Col BH.342C/DHS1 & BH.342C/DHS1 & 9.1 & - \\
\hline
\end{tabular}

Table S8. Significant QTLs for precursor-product ratios in ABA catabolites.

\begin{tabular}{|c|c|c|c|c|c|c|c|}
\hline Trait & $\begin{array}{l}\text { Chromo- } \\
\text { some }\end{array}$ & $\begin{array}{c}\mathrm{cM} \\
\text { interval }\end{array}$ & $\begin{array}{c}\text { LOD } \\
\text { (MQM) }\end{array}$ & $\begin{array}{l}\text { Locus } \\
\text { region }\end{array}$ & Nearest marker & $\begin{array}{c}\text { Explained } \\
\text { Variance (\%) }\end{array}$ & $\begin{array}{c}\text { Additive } \\
\text { effect }\end{array}$ \\
\hline ABAGE/ABA & 5 & $107.5^{\sim 117}$ & 5.0 & BF.168L-Col HH.122C/120L & DF.119L & 12 & - \\
\hline \multirow{2}{*}{$\mathrm{ABA} / \mathrm{PA}$} & 1 & $13.1 \sim 30.9$ & 5.0 & HH.335C-Col/PhyA CH.160L-Col & EC. $480 \mathrm{C}$ & 9.3 & - \\
\hline & 4 & $58^{\sim 71}$ & 6.5 & CH.70L/71C-Col BH.342C/DHS1 & HH.159C-Col & 12.4 & - \\
\hline \multirow{2}{*}{ PA/DPA } & 2 & $65.3^{\sim} 70.8$ & 4.4 & DF.140C EC.235L-Col/247C & EC.235L-Col/247C & 7.7 & + \\
\hline & 4 & $58 \sim 68$ & 15.9 & CH.70L/71C-Col BH.342C/DHS1 & HH.159C-Col & 33.8 & + \\
\hline \multirow{3}{*}{ ABA/DPA } & 2 & $65.3 \sim 70.8$ & 8.4 & DF.140C EC.235L-Col/247C & EC.235L-Col/247C & 15.0 & + \\
\hline & 3 & $25.1 \sim 43.1$ & 4.5 & GH.390L BH.225C-Col & GB.210L & 7.6 & - \\
\hline & 4 & $58 \sim 69$ & 12.5 & CH.70L/71C-Col BH.342C/DHS1 & HH.159C-Col & 23.9 & + \\
\hline \multirow[t]{2}{*}{ ABAGE/PA } & 3 & $0 \sim 6.8$ & 6.9 & DF.77C EG.75L & DF.77C & 17.2 & - \\
\hline & 2 & $65.3^{\sim} 70.8$ & 6.8 & DF.140C EC.235L-Col/247C & EC.235L-Col/247C & 12.2 & + \\
\hline \multirow[t]{2}{*}{ ABAGE/DPA } & 3 & $31.4 \sim 41.1$ & 6.3 & EC. $83 \mathrm{C} / 84 \mathrm{~L} \sim \mathrm{BH} .225 \mathrm{C}-\mathrm{Col}$ & GB.210L & 11.2 & - \\
\hline & 4 & $59 \sim 69$ & 10.9 & CH.70L/71C-Col BH.342C/DHS1 & HH.159C-Col & 21.1 & + \\
\hline
\end{tabular}


Chapter 5

Table S9. Significant QTLs for substrate-product ratios in CKs.

\begin{tabular}{|c|c|c|c|c|c|c|c|}
\hline Trait & $\begin{array}{c}\text { Chromo- } \\
\text { some }\end{array}$ & $\begin{array}{c}\mathrm{cM} \\
\text { interval }\end{array}$ & $\begin{array}{c}\text { LOD } \\
\text { (MQM) }\end{array}$ & $\begin{array}{l}\text { Locus } \\
\text { region }\end{array}$ & $\begin{array}{l}\text { Nearest } \\
\text { marker }\end{array}$ & $\begin{array}{c}\text { Explained } \\
\text { Variance (\%) }\end{array}$ & $\begin{array}{c}\begin{array}{c}\text { Additive } \\
\text { effect }\end{array} \\
\end{array}$ \\
\hline $\mathrm{iPR} / \mathrm{iP}$ & 3 & $19.1 \sim 38.7$ & 2.8 & BF.270L-Col/271C GB.210L & AD.92L & 7.8 & - \\
\hline \multirow{2}{*}{ iP/iP9G } & \multirow{2}{*}{3} & $0 \sim 8.8$ & 5.3 & DF.77C EG.75L & DF.77C & 13.1 & + \\
\hline & & $61.2 \sim 85.8$ & 2.7 & GD.296C-Col HH.90L-Col & HH.171C-Col/173L & 6.4 & - \\
\hline iPRMP/iP & 3 & $19.1 \sim 38.7$ & 2.8 & BF.270L-Col/271C $\sim$ GB.210L & AD.92L & 7.8 & - \\
\hline \multirow{2}{*}{ iPRMP/tZRMP } & 2 & $33.4 \sim 55.6$ & 2.5 & CH.65C GD.298C & FD.85C & 6.4 & - \\
\hline & 3 & $0 \sim 13.1$ & 2.9 & DF.77C FD.111L-Col/136C & DF.77C & 7.5 & - \\
\hline $\mathrm{iPR} / \mathrm{tZR}$ & 5 & $20.5 \sim 57.5$ & 2.6 & BH.107L-Col BH.96L-Col & AD.129L-Col & 6.0 & + \\
\hline \multirow{5}{*}{$\mathrm{iP} / \mathrm{tZ}$} & 1 & $0 \sim 14.1$ & 5.9 & PVV4 EC.480C & PVV4 & 12.6 & - \\
\hline & \multirow{2}{*}{. } & $0 \sim 17.9$ & 3.5 & AD.156C FD.81L & GH.580L & 7.7 & - \\
\hline & & $32.4 \sim 52.2$ & 4.6 & CH.65C GD.298C & FD.85C & 10.1 & - \\
\hline & \multirow{2}{*}{3} & $20.1 \sim 36.7$ & 3.2 & BF.270L-Col GB.210L & GH.390L & 6.6 & + \\
\hline & & $80.4 \sim 85.8$ & 3.1 & HH.171C-Col HH.9OL-Col & HH.9OL-Col & 6.5 & + \\
\hline $\mathrm{tZRMP} / \mathrm{tZ}$ & 3 & $7.8 \sim 38.7$ & 3.6 & EG.75L GB.210L & $\mathrm{EC} .83 \mathrm{C} / 84 \mathrm{~L}$ & 10.4 & + \\
\hline tZ/tZOG & 2 & $42.4 \sim 58.2$ & 5.4 & FD.85C BH.120L-Col & Erecta/GPA1 & 14.7 & - \\
\hline \multirow{3}{*}{$\mathrm{tZ} / \mathrm{tZ7G}$} & 1 & $0 \sim 37.7$ & 2.9 & PVV4 AD.106L-Col & PVV4 & 5.3 & + \\
\hline & 3 & $22.1 \sim 31.4$ & 9.7 & BF.270L-Col/271C AD.92L & GH.390L & 19.7 & - \\
\hline & 4 & $58 \sim 80.2$ & 3.3 & CH.70L/71C-Col BH.342C/DHS1 & BH.342C/DHS1 & 6.0 & + \\
\hline \multirow{2}{*}{ tZ/tZ9G } & 1 & $1 \sim 20.3$ & 4.1 & PPV4 EC.66C & PVV4 & 8.6 & + \\
\hline & 3 & $20.1 \sim 30.4$ & 8.7 & BF.270L/271C AD.92L & $\mathrm{EC} .83 \mathrm{C} / 84 \mathrm{~L}$ & 19.6 & - \\
\hline $\mathrm{tZ7G/tZ9G}$ & 3 & $85 \sim 85.8$ & 25.8 & BH.109L-Col HH.9OL-Col & HH.9OL-Col & 54.9 & + \\
\hline DZ7G/DZ9G & 3 & $82.4 \sim 85.8$ & 14.7 & HH.171C-Col/173L HH.90L-Col & HH.9OL-Col & 36.5 & + \\
\hline cZRMP/cZR & 1 & $98.3 \sim 127.5$ & 3.0 & CH.215L HH.360L-Col & CD.173L/175C-Col & 8.0 & + \\
\hline $\mathrm{cZR} / \mathrm{cZ}$ & 5 & $77.5^{\sim 94}$ & 3.1 & DFR GB.102L-Col/105C & AD.75C-Col & 7.6 & + \\
\hline
\end{tabular}


Table S10. Significant QTLs for metabolite-ratios between different groups of hormones. ${ }^{\S}$ The superscripts indicate QTL regions newly detected from hormone-hormone ratios, at which any locus for single hormones in pair is not observed.

\begin{tabular}{|c|c|c|c|c|c|c|c|}
\hline Trait & $\begin{array}{c}\text { Chromo- } \\
\text { some }\end{array}$ & $\begin{array}{c}\mathrm{cM} \\
\text { interval }\end{array}$ & $\begin{array}{c}\text { LOD } \\
\text { (MQM) }\end{array}$ & $\begin{array}{l}\text { Locus } \\
\text { region }\end{array}$ & $\begin{array}{l}\text { Nearest } \\
\text { marker }\end{array}$ & $\begin{array}{c}\text { Explained } \\
\text { Variance (\%) }\end{array}$ & $\begin{array}{c}\text { Additive } \\
\text { effect }\end{array}$ \\
\hline \multirow{3}{*}{ IAA/iP } & 1 & $27.3 \sim 37.3$ & 5.3 & GD.86L AD.106L-Col & CC.98L-Col/101C & 12.6 & - \\
\hline & 2 & $65.3^{\sim} 70.8$ & 3.1 & DF.140C EC.235L-Col/247C & EC.235L-Col/247C & 6.9 & + \\
\hline & 3 & $0 \sim 5.8$ & 2.7 & DF.77C EG.75L & DF.77C & 6.2 & + \\
\hline \multirow{2}{*}{ IAA/tZ } & \multirow{2}{*}{1} & $0 \sim 13.1$ & 5.2 & PVV4 EC. $480 C$ & PVV4 & 10 & - \\
\hline & & $26.3 \sim 34.7$ & 4.7 & GD.86L AD.106L-Col & CC.98L-Col/101C & 4.7 & - \\
\hline $\mathrm{IAA} / \mathrm{cZ}$ & 1 & $22.3^{\sim} 37.7$ & 4.2 & EC.480C AD.106L-Col & CC.98L-Col/101C & 11.7 & - \\
\hline IAA/ABAGE & 2 & $66.3^{\sim} 70.8$ & 6.0 & DF.140C EC.235L-Col/247C & EC.235L-Col/247C & 15.2 & + \\
\hline \multirow{2}{*}{ IAA/ABA } & 2 & $66.3^{\sim} 70.8$ & 4.1 & DF.140C EC.235L-Col/247C & EC.235L-Col/247C & 10.2 & + \\
\hline & 3 & $4.8 \sim 22.1$ & 3.3 & GB.120C-Col/GAPC GH.390L & FD.111L-Col/136C & 7.9 & - \\
\hline \multirow{3}{*}{ IAA/PA } & 2 & $66.3^{\sim} 70.8$ & 3.7 & DF.140C EC.235L-Col/247C & EC.235L-Col/247C & 8.6 & + \\
\hline & 3 & $8.8 \sim 23.1$ & 5.6 & EG.75L GH.390L & FD.111L-Col/136C & 13 & - \\
\hline & 4 & $49.1^{\sim} 73^{\S}$ & 2.8 & CD.329C-Col/CH42 BH.342C/DHS1 & HH.159C-Col & 6.3 & - \\
\hline \multirow{4}{*}{ IAA/DPA } & 2 & $68.3^{\sim} 70.8$ & 11.0 & DF.140C EC.235L-Col/247C & EC.235L-Col/247C & 20.9 & + \\
\hline & \multirow{2}{*}{3} & $13.1^{\sim} 22.1^{\S}$ & 3.9 & FD.111L-Col/136C GH.390L & BF.270L-Col/271C & 7.3 & - \\
\hline & & $27.1 \sim 42.1$ & 3.6 & $\mathrm{EC} .83 \mathrm{C} / 84 \mathrm{~L} \sim \mathrm{BH} .225 \mathrm{C}-\mathrm{Col}$ & GB.210L & 6 & - \\
\hline & 4 & $58 \sim 71$ & 6.7 & CH.70L/71C-Col BH.342C/DHS1 & HH.159C-Col & 11.8 & + \\
\hline \multirow{2}{*}{ IAA/SA } & 1 & $23.5^{\sim 36.7}$ & 6.6 & EC.66C AD.106L-Col & CC.98L-Col/101C & 15.1 & - \\
\hline & 2 & $66.3^{\sim} 70.8$ & 5.8 & DF.140C EC.235L-Col/247C & EC.235L-Col/247C & 13.2 & + \\
\hline JA/PA & 3 & $0 \sim 17.1$ & 3.0 & DF.77C BF.270L-Col/271C & GB.120C-Col/GAPC & 8.7 & - \\
\hline \multirow{2}{*}{ JA/DPA } & 2 & $60.2^{\sim} 70.8$ & 3.4 & BH.120L-Col EC.235L-Col/247C & EC.235L-Col/247C & 8.5 & + \\
\hline & 4 & $49.1 \sim 76$ & 5.1 & CD.329C-Col/CH42 BH.342C/DHS1 & HH.159C-Col & 13.2 & + \\
\hline \multirow{2}{*}{ SA/PA } & 3 & $0 \sim 16.1$ & 4.0 & DF.77C BF.270L-Col/271C & DF.77C & 10.4 & - \\
\hline & 4 & $36.1^{\sim} 75^{\S}$ & 2.8 & CD.84C-Col/85L BH.342C/DHS1 & HH.159C-Col & 7 & - \\
\hline \multirow{3}{*}{ SA/DPA } & 2 & $66.3^{\sim} 70.8$ & 5.8 & DF.140C EC.235L-Col/247C & EC.235L-Col/247C & 11.7 & + \\
\hline & 3 & $23.1 \sim 44.1$ & 3.6 & GH.390L BH.225C-Col & GB.210L & 6.9 & - \\
\hline & 4 & $59 \sim 72$ & 9.7 & CH.70L/71C-Col BH.342C/DHS1 & HH.159C-Col & 20.5 & + \\
\hline
\end{tabular}





\section{Chapter 6}

General Discussion 
Plants as sedentary organisms have the ability to readily react to changes of the environment during growth and developmental processes. Roots and shoots communicate to optimize growth in response to internal and external stimuli. This communication goes beyond the supply of photosynthates from shoots to roots, and that of water and nutrients in the other direction. Sensing and communication among cells, tissues and organs often depend on chemical messengers, the plant hormones, moving from one part to another. Some of the plant hormones are synthesized in one tissue and act on target sites via transport mechanisms, but most plant hormones also act on neighbouring cells in the vicinity of the site of biosynthesis.

Hormone metabolism in plants shows complexities of metabolic redundancy and plasticity to maintain hormone levels in response to environmental changes and developmental conditions, referred to as hormone homeostasis. The process of hormone metabolism is at least partly dependent on the genetic make-up of the plants.

The variation of quantitative traits in populations is largely contributed by genetic variation of DNA sequences. In the post-genome era accumulated resources of molecular markers and DNA polymorphisms have been developed in Arabidopsis thaliana and several other plant species. Linkage mapping based on bi-parental populations has been used as a genetic tool for detecting alleles that have a large effect on the phenotypic traits of interest (Sergeeva et al., 2006). More recently, genomewide-association (GWA) mapping, a powerful approach to provide high mapping resolution, has been extensively applied to many plant species (Bergelson and Roux, 2010). These two main techniques of quantitative genetics have been used to elucidate biochemical pathways underlying primary and secondary metabolites in several plants (Fernie and Tohge, 2017). However, to date as far as I know, no study has been done in quantitative genetics of hormone levels as phenotypic traits in Arabidopsis or other plant species.

In this General Discussion, I will first discuss the importance of using proper methods in the quantitative analysis of hormones, which is crucial for the reliability of data throughout all chapters. Subsequently I will discuss how to assess results of QTL analysis and how to continue with this type of research.

\section{Accuracy of hormone measurements}

Chapter two describes a validation study for simultaneous analysis of a range of different plant hormones using UPLC-ESI-MS/MS. Accurate quantitative data of actual levels of metabolites are a prerequisite for success in natural variation studies and further analysis of the genetic and biochemical bases of variation. In addition, I wanted to reduce sample size as much as possible to reduce sample preparation costs. As I also wanted to improve our understanding of hormone-hormone interactions, 
it was important for me to target multiple groups of plant hormones. This approach would create the possibility that I could find overlapping QTLs for different hormones, pointing at joint regulation.

I anticipated that it would be challenging to analyse different groups of hormones using a single SPE cartridge, and at the same time attain a nearly zero level of matrix interference. In the present study, the hydrophilic-lipophilic balance (HLB) sorbent that has a balanced ratio of two monomers, divinylbenzene and $N$-vinylpyrrolidine, was finally selected for the rest of the experiment using the full set of lines of the RIL population. A drawback of this material is the limited capacity of capturing and retaining ionic analytes. Two major concerns, impurities and recovery rates, seemed to influence each other, but understanding their relations is complicated. As discussed in Chapter 2, values of recovery rates of the analytes in the semi-purified samples did not represent their real recovery rates. For example, in Fig. 2 in Chapter 2, none of the SPE sorbents was really better than the others, because recovery rates themselves do not provide information on matrix interference caused by impurities. When ion suppression and enhancement are sufficiently controlled, we can assure that true recovery rates of analytes are obtained, and consequently, that quantitative data are reliable.

It is remarkable that some articles for plant hormone analysis using LC-MS seem to consider matrix effects of impurities negligible in quantitative determination (Cai et al., 2016; Durgbanshi et al., 2005; Pan et al., 2010; Segarra et al., 2006). Those authors determined quantities of plant hormones in crude extracts without suitable purification such as solid phase extraction. When levels of hormones are determined by UV-Visible spectrophotometry, matrix effects are irrelevant. But it is different when an LC-MS system is applied for the analysis. Comparatively, although LC-ESIMS/MS provides high sensitivity and selectivity through the use of mass/charge $(\mathrm{m} / \mathrm{z})$ of fragment ions of the analytes, signal intensities presented in chromatograms are likely to be influenced dramatically by impurities, especially polar compounds (Bonfiglio et al., 1999). Therefore, the concern of matrix effects in LC-MS should be properly evaluated in the process of method development, in order to attain a close-to-zero-level of ion suppression/enhancement for the analytes.

Natural variation of a quantitative trait is the sum of genetic and non-genetic factors. If inaccuracy of the method of hormone quantification increases, the non-genetic factors (e.g., environmental perturbation and experimental errors) will have a great influence on the quality of data, eventually decreasing the chance of finding genetic elements. In Chapter 3, I illustrated that natural variation for the levels of endogenous hormones is low, so that the use of a suitable purification protocol is critical and a prerequisite for QTL analysis. The accuracy of the quantitative data I generated is indirectly supported by the detection of significant QTLs for several hormones and confirmations of these QTLs using NILs in Chapter 4. 


\section{Choice of populations}

Chapter 3 describes natural variation in hormone levels in the roots of 13 Arabidopsis accessions and interprets the roles of these hormone levels in the process of shaping root phenotypes. I found that variation in the biologically active trans-zeatin and IAA were very limited, within the tested set of accessions. However, inactive intermediates e.g., tZGs in CK, showed relatively large variations (Fig. 5 in Chapter 3). Regarding these results, I already discussed a limitation of quantitative analysis based on a whole-root extract that may obscure a possible differential distribution of hormones on the cellular or tissue level. Additionally, it is likely that genetic alleles for biologically active forms of hormone are highly conserved. This assumption was a reason that I preferred conventional linkage mapping as the start of quantitative genetic analysis rather than association mapping. It is also relevant that association mapping, in general, is less efficient than linkage mapping when the genetic diversity in a species is low, as seems to be the case for hormones. On the other hand, association mapping based on natural populations reflecting the long history of recombination events provide high resolution, down to the gene level and works well when more allelic variation is expected for the trait of interest (Fernie and Tohge, 2017).

Both approaches (association- and linkage mapping) are often complementary. A bi-parental population seemed to be less risky for my study than natural collections because of several reasons, as reviewed in Myles et al. (2009). First of all, bi-parental populations consist of a relatively small number of lines, as compared to association populations. This makes the experiment easier to handle. Indeed a collection of 360 Arabidopsis accessions was established and used for many association studies, considerably more than the 152 lines of the Ler $\times$ Cvi RIL population used in my study. Secondly, Ler and Cvi seemed to be divergent accessions for variations in hormone levels (Chapter 3), suggesting that they may contain rarely occurring alleles that have a large effect on my traits of interest. In addition there was also a criterion that association mapping has a reduced power to detect a QTL when rare alleles or weak-effect alleles are involved.

\section{Duration of physiological antagonism between IAA and CK for root growth}

Principal component analysis (PCA) is often used to interpret possible interactions between variables (traits) in different groups of phenotypic characteristics. In Figure 6 of Chapter 3, I suggested physiological interactions between hormones and root phenotypic traits by comparing accumulated knowledge of hormone functions for root system architecture. A limitation of Figure 6 in Chapter 3 is that the analysis has been carried out in a specific time point (23-day-old roots) rather than on several different growth stages during the whole plant life. In order to assess the effect of developmental stage, I compared the results of Chapter 3 with data from the older plants, obtained with the Ler $\times$ Cvi RIL population, harvested at 35 days. In the $35 \mathrm{~d}$ old plants, root fresh weight (RFW), IAA and tZ showed 
antagonistic relationships (Fig. 1), similar as in 23-day-old roots of 13 natural accessions. This antagonistic relationship was also observed in two different sub-populations, vegetative lines and flowering lines of the RILs (data not shown). However, there were slight changes in the topology between IAA and $\mathrm{tZ}$ for RFW in quadrants of Cartesian coordinate system (X and Y). Approximately, $40 \%$ of the variation was explained by the first two principal components, which was lower than $58 \%$ of the variation in 23-day-old roots of 13 natural accessions. This suggests that hormonal interactions for root growth gradually change with plant development.

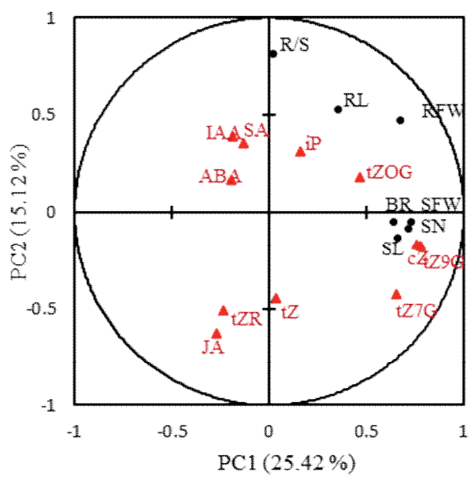

Figure 1. PCA of hormones and phenotypic traits in a Ler $\times$ Cvi RIL population. Abbreviations of observable phenotypic traits follow as: R/S, root-shoot weight ratio; RL, root length; RFW, root fresh weight; $\mathrm{BR}$, bolting rate; SFW, shoot fresh weight; SN, shoot branch number; SL, shoot length.

\section{Numbers of significant QTLs found between different organs}

In Chapter 4, I pointed out that the average number of significant QTLs per hormonal compound in roots was relatively small. However, in Chapter 5 the analysis of rosette leaves showed the existence of more QTLs for individual hormones. What caused this difference, despite that the harvest time was the same? Does it mean that more genes are expressed for hormone metabolism in the leaf? In the root system the missing heritability for levels of biologically active hormones suggests that the variation in a single gene cannot account for much of the variation of the phenotypic trait and rare but large-effect alleles do not exist in the parental lines (Manolio et al., 2009). In other words, it is hard to detect a responsible QTL when the rare and large-effect allele driven by a parental line exists but is poorly expressed at the time point of sampling. The QTL analysis using two sub-populations (vegetative and flowering lines) revealed that in many cases QTLs were composed of more than one locus, functional for either vegetative or flowering stage. Taken together, it is recommended that QTL analysis for hormone levels should be conducted at both the early stage of development, when all lines of the RIL 
are vegetative, and the later stage when all lines are flowering. I envisage that these 4 sets of QTL analysis according to distinct time points of development and different organs would provide a better systematic understanding of the genetic regulation of hormone metabolism.

It has been suggested that the majority of metabolite traits display additive and dominant modes of inheritance, wherein primary metabolite abundance is dominantly and secondary metabolite abundance recessively inherited (Fernie and Tohge, 2017). Alseekh et al. (2015) demonstrated that in tomato the variance for secondary metabolites is greater but the number of loci responsible for a given metabolite is far fewer than for primary metabolites. I found that the variation in hormone quantities was small and in general the number of QTLs responsible for these levels was low. In hormone metabolism, it is interesting that variation in hormone levels was limited like the case of primary metabolites, although most of them are generated in biosynthetic pathways separately from primary metabolism.

\section{QTL of interest in ABA catabolism}

For the levels of DPA as an ABA catabolite, I paid attention to two large-effect QTLs that were found on the bottom of chromosome 2 and on the lower arm of chromosome 4 (Fig. 4 and Table S5 in Chapter 5). These QTLs are interesting target for a following-up study because they are present in both the vegetative and flowering stage. In ABA catabolism, there are two main pathways for $\mathrm{ABA}$ inactivation (Fig. 2): one is hydroxylation by a cytochrome P450 and the other is the conversion to a glucose ester by an ABA glucosyltransferase. Except for CYP707A, to date none of the genes encoding for the biosynthetic pathway beyond 8 '-hydroxy-ABA has been elucidated (Endo et al., 2014). It is possible that this would include a so far unidentified reductase for the production of DPA from its precursor(s). The QTLs for the levels of the ABA catabolites, DPA, found in this study may provide opportunities to identify the missing gene(s) involved in this catabolism. Confirmation of the QTLs detected using NILs will be necessary and should be followed by fine mapping using a population derived from a cross between Ler and a selected NIL covering the whole significant QTL region. 


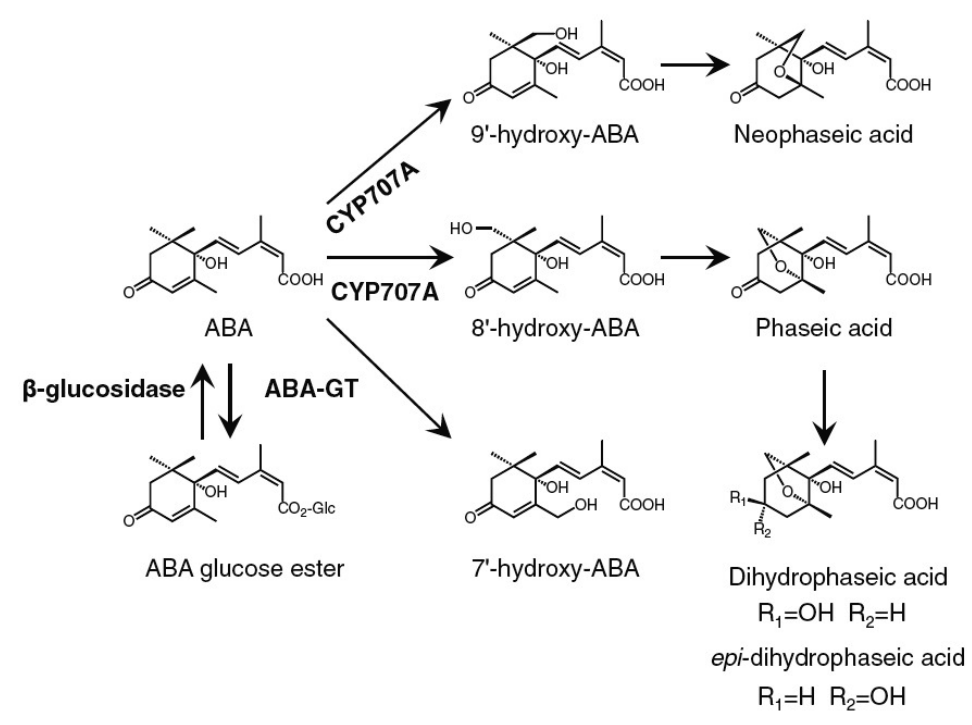

Figure 2. ABA catabolic pathway in higher plants. The drawing was adopted from (Endo et al., 2014).

\section{Perspective remarks}

Metabolite profiling has extensively been used to study the regulation of the accumulation of primary and secondary metabolites. I would like to exemplify the study of Keurentjes et al. (2006) as an outstanding study of metabolic QTL analysis for a huge number of analytes in the same RIL population. By virtue of such efforts through traditional linkage mapping analysis, considerable understanding in metabolic regulation has been achieved. Association mapping analysis has also been enthusiastically used in the study of plant metabolic regulation (Fang et al., 2019). The major advantage of association mapping exists in its high resolution power of QTL in the broad genomic region because genotypic data of genome-wide-association mapping obtained from hundreds of natural lines provides a better picture of species-wide allelic diversity, frequency, and combination than linkage mapping does. Actually only a very small portion of QTL has been cloned even after countless loci were found in plants.

In order to detect more distinct QTLs responsible for hormone metabolism, quantitative analysis as a further study can be done based on different tissues. For example, sampling the region of root tip only, may increase the chance of finding hormonal QTLs that were not found in samples of entire root systems.

My study showed that hormonal QTLs could be mapped to known responsible loci/genes, (e.g., cytokinin $N$-glucosyltransferase), and also revealed new loci, in Arabidopsis. Hormone QTL mapping 
is likely also applicable to populations of other plant species (Cardoso et al., 2014). A possible suggestion is that natural variation studies using more extended Arabidopsis ecotypes can be designed to uncover lines with extreme hormone metabolism phenotypes, subsequently followed by linkage mapping analysis with a suitable segregating population. On the other hand, association panels and RIL populations should be regarded as complements to each other. For quantitative genetics of hormone metabolism, acquisition of more diverse chemical standards is also important to refine quantitative data covering each metabolite in complex biosynthetic pathways. 


\section{References}

Acharya B, Assmann S. 2009. Hormone interactions in stomatal function. Plant Molecular Biology 69, 451462.

Aloni R, Aloni E, Langhans M, Ullrich CI. 2006. Role of cytokinin and auxin in shaping root architecture: Regulating vascular differentiation, lateral root initiation, root apical dominance and root gravitropism. Annals of Botany 97, 883-893.

Alonso-Blanco C, Aarts MG, Bentsink L, Keurentjes JJ, Reymond M, Vreugdenhil D, Koornneef M. 2009. What has natural variation taught us about plant development, physiology, and adaptation? Plant Cell 21, 1877-1896.

Alonso-Blanco C, Blankestijn-de Vries H, Hanhart CJ, Koornneef M. 1999. Natural allelic variation at seed size loci in relation to other life history traits of Arabidopsis thaliana. Proceedings of the National Academy of Sciences of the United States of America 96, 4710-4717.

Alonso-Blanco C, El-Assal SED, Coupland G, Koornneef M. 1998a. Analysis of natural allelic variation at flowering time loci in the Landsberg erecta and Cape Verde islands ecotypes of Arabidopsis thaliana. Genetics 149, 749-764.

Alonso-Blanco C, Mendez-Vigo B. 2014. Genetic architecture of naturally occurring quantitative traits in plants: an updated synthesis. Current Opinion in Plant Biology 18, 37-43.

Alonso-Blanco C, Peeters AJM, Koornneef M, Lister C, Dean C, van den Bosch N, Pot J, Kuiper MTR. 1998b. Development of an AFLP based linkage map of Ler, Col and Cvi Arabidopsis thaliana ecotypes and construction of a Ler/Cvi recombinant inbred line population. Plant Journal 14, 259-271.

Alseekh S, Tohge T, Wendenberg R, Scossa F, Omranian N, Li J, Kleessen S, Giavalisco P, Pleban T, Mueller-Roeber B, Zamir D, Nikoloski Z, Fernie AR. 2015. Identification and mode of inheritance of quantitative trait loci for secondary metabolite abundance in tomato. Plant Cell 27, 485-512.

Alvim R, Thomas S, Saunders PF. 1978. Seasonal-Variation in Hormone Content of Willow .2. Effect of Photoperiod on Growth and Abscisic-Acid Content of Trees under Field Conditions. Plant Physiology 62, 779780.

Annesley TM. 2003. Ion suppression in mass spectrometry. Clinical Chemistry 49, 1041-1044.

Antoniadi I, Plackova L, Simonovik B, Dolezal K, Turnbull C, Ljung K, Novak O. 2015. Cell-TypeSpecific Cytokinin Distribution within the Arabidopsis Primary Root Apex. Plant Cell 27, 1955-1967.

Armengaud P, Zambaux K, Hills A, Sulpice R, Pattison RJ, Blatt MR, Amtmann A. 2009. EZ-Rhizo: integrated software for the fast and accurate measurement of root system architecture. Plant Journal 57, 945956.

Atwell S, Huang YS, Vilhjalmsson BJ, Willems G, Horton M, Li Y, Meng DZ, Platt A, Tarone AM, Hu TT, Jiang R, Muliyati NW, Zhang X, Amer MA, Baxter I, Brachi B, Chory J, Dean C, Debieu M, de Meaux J, Ecker JR, Faure N, Kniskern JM, Jones JDG, Michael T, Nemri A, Roux F, Salt DE, Tang CL, Todesco M, Traw MB, Weigel D, Marjoram P, Borevitz JO, Bergelson J, Nordborg M. 2010. Genome-wide association study of 107 phenotypes in Arabidopsis thaliana inbred lines. Nature 465, 627-631.

Bai YL, Cai BD, Luo XT, Ye TT, Feng YQ. 2018. Simultaneous Determination of Abscisic Acid and Its Catabolites by Hydrophilic Solid-Phase Extraction Combined with Ultra High Performance Liquid Chromatography-Tandem Mass Spectrometry. Journal of Agricultural and Food Chemistry 66, 10906-10912.

Bari R, Jones J. 2009. Role of plant hormones in plant defence responses. Plant Molecular Biology 69, 473488.

Bartrina I, Otto E, Strnad M, Werner T, Schmulling T. 2011. Cytokinin Regulates the Activity of Reproductive Meristems, Flower Organ Size, Ovule Formation, and Thus Seed Yield in Arabidopsis thaliana. Plant Cell 23, 69-80. 
Bentsink L, Hanson J, Hanhart CJ, Blankestijn-de Vries H, Coltrane C, Keizer P, El-Lithy M, AlonsoBlanco C, de Andres MT, Reymond M, van Eeuwijk F, Smeekens S, Koornneef M. 2010. Natural variation for seed dormancy in Arabidopsis is regulated by additive genetic and molecular pathways. Proceedings of the National Academy of Sciences of the United States of America 107, 4264-4269.

Bentsink L, Yuan K, Koornneef M, Vreugdenhil D. 2003. The genetics of phytate and phosphate accumulation in seeds and leaves of Arabidopsis thaliana, using natural variation. Theoretical and Applied Genetics 106, 1234-1243.

Bergelson J, Roux F. 2010. Towards identifying genes underlying ecologically relevant traits in Arabidopsis thaliana. Nat Rev Genet 11, 867-879.

Bian YL, Gu X, Sun DL, Wang YJ, Yin ZT, Deng DX, Wang YQ, Li GS. 2015. Mapping dynamic QTL of stalk sugar content at different growth stages in maize. Euphytica 205, 85-94.

Bielach A, Podlesakova K, Marhavy P, Duclercq J, Cuesta C, Muller B, Grunewald W, Tarkowski P, Benkova E. 2012. Spatiotemporal Regulation of Lateral Root Organogenesis in Arabidopsis by Cytokinin. Plant Cell 24, 3967-3981.

Bishopp A, Benkova E, Helariutta Y. 2011. Sending mixed messages: auxin-cytokinin crosstalk in roots. Current Opinion in Plant Biology 14, 10-16.

Bonfiglio R, King RC, Olah TV, Merkle K. 1999. The effects of sample preparation methods on the variability of the electrospray ionization response for model drug compounds. Rapid Communications in Mass Spectrometry 13, 1175-1185.

Borevitz JO, Maloof JN, Lutes J, Dabi T, Redfern JL, Trainer GT, Werner JD, Asami T, Berry CC, Weigel D, Chory J. 2002. Quantitative trait loci controlling light and hormone response in two accessions of Arabidopsis thaliana. Genetics 160, 683-696.

Brachi B, Faure N, Horton M, Flahauw E, Vazquez A, Nordborg M, Bergelson J, Cuguen J, Roux F. 2010. Linkage and Association Mapping of Arabidopsis thaliana Flowering Time in Nature. Plos Genetics 6.

Brunoud G, Wells DM, Oliva M, Larrieu A, Mirabet V, Burrow AH, Beeckman T, Kepinski S, Traas J, Bennett MJ, Vernoux T. 2012. A novel sensor to map auxin response and distribution at high spatio-temporal resolution. Nature 482, 103-U132.

Buntjer JB, Sorensen AP, Peleman JD. 2005. Haplotype diversity: the link between statistical and biological association. Trends in Plant Science 10, 466-471.

Cai WJ, Ye TT, Wang Q, Cai BD, Feng YQ. 2016. A rapid approach to investigate spatiotemporal distribution of phytohormones in rice. Plant Methods 12, 47.

Cao J, Schneeberger K, Ossowski S, Gunther T, Bender S, Fitz J, Koenig D, Lanz C, Stegle O, Lippert C, Wang X, Ott F, Muller J, Alonso-Blanco C, Borgwardt K, Schmid KJ, Weigel D. 2011. Whole-genome sequencing of multiple Arabidopsis thaliana populations. Nature Genetics 43, 956-U960.

Cardoso C, Zhang Y, Jamil M, Hepworth J, Charnikhova T, Dimkpa So, Meharg C, Wright MH, Liu J, Meng X, Wang Y, Li J, McCouch SR, Leyser O, Price AH, Bouwmeester HJ, Ruyter-Spira C. 2014. Natural variation of rice strigolactone biosynthesis is associated with the deletion of two MAX1 orthologs. Proceedings of the National Academy of Sciences of the United States of America 111, 2379-2384.

Carreno-Quintero N, Acharjee A, Maliepaard C, Bachem CWB, Mumm R, Bouwmeester H, Visser RGF, Keurentjes JJB. 2012. Untargeted Metabolic Quantitative Trait Loci Analyses Reveal a Relationship between Primary Metabolism and Potato Tuber Quality. Plant Physiology 158, 1306-1318.

Chambers E, Wagrowski-DiehI DM, Lu ZL, Mazzeo JR. 2007. Systematic and comprehensive strategy for reducing matrix effects in LC/MS/MS analyses. Journal of Chromatography B-Analytical Technologies in the Biomedical and Life Sciences 852, 22-34.

Chandler JW. 2009. Auxin as compere in plant hormone crosstalk. Planta 231, 1-12.

Chayut N, Yuan H, Ohali S, Meir A, Yeselson Y, Portnoy V, Zheng Y, Fei ZJ, Lewinsohn E, Katzir N,

Schaffer AA, Gepstein S, Burger J, Li L, Tadmor Y. 2015. A bulk segregant transcriptome analysis reveals 
metabolic and cellular processes associated with Orange allelic variation and fruit beta-carotene accumulation in melon fruit. Bmc Plant Biology 15.

Chen W, Gao Y, Xie W, Gong L, Lu K, Wang W, Li Y, Liu X, Zhang H, Dong H, Zhang W, Zhang L, Yu S, Wang G, Lian $\mathbf{X}$, Luo J. 2014. Genome-wide association analyses provide genetic and biochemical insights into natural variation in rice metabolism. Nat Genet 46, 714-721.

Chen WQ, Gai Y, Liu SC, Wang RX, Jiang XN. 2010. Quantitative Analysis of Cytokinins in Plants by High Performance Liquid Chromatography: Electronspray Ionization Ion Trap Mass Spectrometry. Journal of Integrative Plant Biology 52, 925-932.

Chiwocha SDS, Abrams SR, Ambrose SJ, Cutler AJ, Loewen M, Ross ARS, Kermode AR. 2003. A method for profiling classes of plant hormones and their metabolites using liquid chromatography-electrospray ionization tandem mass spectrometry: an analysis of hormone regulation of thermodormancy of lettuce (Lactuca sativa L.) seeds. Plant Journal 35, 405-417.

Clark RM, Schweikert G, Toomajian C, Ossowski S, Zeller G, Shinn P, Warthmann N, Hu TT, Fu G, Hinds DA, Chen HM, Frazer KA, Huson DH, Schoelkopf B, Nordborg M, Raetsch G, Ecker JR, Weigel D. 2007. Common sequence polymorphisms shaping genetic diversity in Arabidopsis thaliana. Science 317, 338342.

Clark RT, Famoso AN, Zhao KY, Shaff JE, Craft EJ, Bustamante CD, Mccouch SR, Aneshansley DJ, Kochian LV. 2013. High-throughput two-dimensional root system phenotyping platform facilitates genetic analysis of root growth and development. Plant Cell and Environment 36, 454-466.

Cubillos FA, Coustham V, Loudet O. 2012. Lessons from eQTL mapping studies: non-coding regions and their role behind natural phenotypic variation in plants. Current Opinion in Plant Biology 15, 192-198.

Davis PJ. 2010. Plant hormones; biosynthesis, signal transduction, action. Springer.

De Smet I, White PJ, Bengough AG, Dupuy L, Parizot B, Casimiro I, Heidstra R, Laskowski M, Lepetit M, Hochholdinger F, Draye X, Zhang HM, Broadley MR, Peret B, Hammond JP, Fukaki H, Mooney S, Lynch JP, Nacry P, Schurr U, Laplaze L, Benfey P, Beeckman T, Bennett M. 2012. Analyzing Lateral Root Development: How to Move Forward. Plant Cell 24, 15-20.

Delker C, PoschI Y, Raschke A, Ullrich K, Ettingshausen S, Hauptmann V, Grosse I, Quint M. 2010. Natural Variation of Transcriptional Auxin Response Networks in Arabidopsis thaliana. Plant Cell 22, 2184-2200. Dello Ioio R, Nakamura K, Moubayidin L, Perilli S, Taniguchi M, Morita MT, Aoyama T, Costantino P, Sabatini S. 2008. A Genetic Framework for the Control of Cell Division and Differentiation in the Root Meristem. Science 322, 1380-1384.

Dobon A, Canet JV, Perales L, Tornero P. 2011. Quantitative genetic analysis of salicylic acid perception in Arabidopsis. Planta 234, 671-684.

Dobrev PI, Havlicek L, Vagner M, Malbeck J, Kaminek M. 2005. Purification and determination of plant hormones auxin and abscisic acid using solid phase extraction and two-dimensional high performance liquid chromatography. Journal of Chromatography A 1075, 159-166.

Dubrovsky JG, Forde BG. 2012. Quantitative Analysis of Lateral Root Development: Pitfalls and How to Avoid Them. Plant Cell 24, 4-14.

Durgbanshi A, Arbona V, Pozo O, Miersch O, Sancho JV, Gomez-Cadenas A. 2005. Simultaneous determination of multiple phytohormones in plant extracts by liquid chromatography-electrospray tandem mass spectrometry. J Agric Food Chem 53, 8437-8442.

El-Din El-Assal S, Alonso-Blanco C, Peeters AJ, Raz V, Koornneef M. 2001. A QTL for flowering time in Arabidopsis reveals a novel allele of CRY2. Nat Genet 29, 435-440.

EI-Lithy ME, Clerkx EJM, Ruys GJ, Koornneef M, Vreugdenhil D. 2004. Quantitative trait locus analysis of growth-related traits in a new Arabidopsis recombinant. Plant Physiology 135, 444-458. 
El-Lithy ME, Reymond M, Stich B, Koornneef M, Vreugdenhil D. 2010. Relation among plant growth, carbohydrates and flowering time in the Arabidopsis Landsberg erecta $\mathrm{x}$ Kondara recombinant inbred line population. Plant Cell Environ 33, 1369-1382.

Endo A, Okamoto M, Koshiba T. 2014. ABA Biosynthetic and Catabolic Pathways. In: Zhang D-P, ed. Abscisic Acid: Metabolism, Transport and Signaling. Dordrecht: Springer Netherlands, 21-45.

Fang C, Fernie AR, Luo J. 2019. Exploring the Diversity of Plant Metabolism. Trends Plant Sci 24, 83-98.

Farre G, Blancquaert D, Capell T, Van Der Straeten D, Christou P, Zhu C. 2014. Engineering complex metabolic pathways in plants. Annu Rev Plant Biol 65, 187-223.

Feng J, Long Y, Shi L, Shi JQ, Barker G, Meng JL. 2012. Characterization of metabolite quantitative trait loci and metabolic networks that control glucosinolate concentration in the seeds and leaves of Brassica napus. New Phytologist 193, 96-108.

Fernie AR, Tohge T. 2017. The Genetics of Plant Metabolism. Annu Rev Genet 51, 287-310.

Fontanals N, Marce RM, Borrull F. 2007. New materials in sorptive extraction techniques for polar compounds. Journal of Chromatography A 1152, 14-31.

Friml J, Vieten A, Sauer M, Weijers D, Schwarz H, Hamann T, Offringa R, Jurgens G. 2003. Effluxdependent auxin gradients establish the apical-basal axis of Arabidopsis. Nature 426, 147-153.

Galweiler L, Guan CH, Muller A, Wisman E, Mendgen K, Yephremov A, Palme K. 1998. Regulation of polar auxin transport by AtPIN1 in Arabidopsis vascular tissue. Science 282, 2226-2230.

Ganal MW, Altmann T, Roder MS. 2009. SNP identification in crop plants. Current Opinion in Plant Biology 12, 211-217.

Garay-Arroyo A, Sanchez MD, Garcia-Ponce B, Azpeitia E, Alvarez-Buylla ER. 2012. Hormone symphony during root growth and development. Developmental Dynamics 241, 1867-1885.

Han YP, Xie DW, Teng WL, Zhang SZ, Chang W, Li WB. 2011. Dynamic QTL analysis of linolenic acid content in different developmental stages of soybean seed. Theoretical and Applied Genetics 122, 1481-1488.

Harpaz-Saad S, Azoulay T, Arazi T, Ben-Yaakov E, Mett A, Shiboleth YM, Hortensteiner S, Gidoni D, Gal-On A, Goldschmidt EE, Eyal Y. 2007. Chlorophyllase is a rate-limiting enzyme in chlorophyll catabolism and is posttranslationally regulated. Plant Cell 19, 1007-1022.

Hasunuma T, Harada K, Miyazawa SI, Kondo A, Fukusaki E, Miyake C. 2010. Metabolic turnover analysis by a combination of in vivo C-13-labelling from (CO2)-C-13 and metabolic profiling with CE-MS/MS reveals rate-limiting steps of the C-3 photosynthetic pathway in Nicotiana tabacum leaves. Journal of Experimental Botany 61, 1041-1051.

Hedden P, Thomas SG. 2012. Gibberellin biosynthesis and its regulation. Biochemical Journal 444, 11-25. Horton MW, Hancock AM, Huang YS, Toomajian C, Atwell S, Auton A, Muliyati NW, Platt A, Sperone FG, Vilhjalmsson BJ, Nordborg M, Borevitz JO, Bergelson J. 2012. Genome-wide patterns of genetic variation in worldwide Arabidopsis thaliana accessions from the RegMap panel. Nat Genet 44, 212-216.

Hou B, Lim EK, Higgins GS, Bowles DJ. 2004. N-glucosylation of cytokinins by glycosyltransferases of Arabidopsis thaliana. J Biol Chem 279, 47822-47832.

Hoyerova K, Gaudinova A, Malbeck J, Dobrev PI, Kocabek T, Solcova B, Travnickova A, Kaminek M. 2006. Efficiency of different methods of extraction and purification of cytokinins. Phytochemistry 67, 11511159.

Huang XH, Zhao Y, Wei XH, Li CY, Wang A, Zhao Q, Li WJ, Guo YL, Deng LW, Zhu CR, Fan DL, Lu YQ, Weng QJ, Liu KY, Zhou TY, Jing YF, Si LZ, Dong GJ, Huang T, Lu TT, Feng Q, Qian Q, Li JY, Han B. 2012. Genome-wide association study of flowering time and grain yield traits in a worldwide collection of rice germplasm. Nature Genetics 44, 32-U53.

Huang XQ, Paulo MJ, Boer M, Effgen S, Keizer P, Koornneef M, van Eeuwijk FA. 2011. Analysis of natural allelic variation in Arabidopsis using a multiparent recombinant inbred line population. Proceedings of the National Academy of Sciences of the United States of America 108, 4488-4493. 
Ikeda Y, Men SZ, Fischer U, Stepanova AN, Alonso JM, Ljung K, Grebe M. 2009. Local auxin biosynthesis modulates gradient-directed planar polarity in Arabidopsis. Nature Cell Biology 11, 731-U770.

Illig T, Gieger C, Zhai GJ, Romisch-Margl W, Wang-Sattler R, Prehn C, Altmaier E, Kastenmuller G, Kato BS, Mewes HW, Meitinger T, de Angelis MH, Kronenberg F, Soranzo N, Wichmann HE, Spector TD, Adamski J, Suhre K. 2010. A genome-wide perspective of genetic variation in human metabolism. Nature Genetics 42, 137-U166.

Ingvarsson PK, Street NR. 2011. Association genetics of complex traits in plants. New Phytologist 189, 909922.

Iyer-Pascuzzi AS, Symonova O, Mileyko Y, Hao YL, Belcher H, Harer J, Weitz JS, Benfey PN. 2010. Imaging and Analysis Platform for Automatic Phenotyping and Trait Ranking of Plant Root Systems. Plant Physiology 152, 1148-1157.

Jaillais Y, Chory J. 2010. Unraveling the paradoxes of plant hormone signaling integration. Nature Structural \& Molecular Biology 17, 642-645.

Jeong Y, Schaffer A, Smith K. 2017. Equilibrium partitioning of organic compounds to OASIS HLB (R) as a function of compound concentration, $\mathrm{pH}$, temperature and salinity. Chemosphere 174, 297-305.

Jessome LL, Volmer DA. 2006. Ion suppression: A major concern in mass spectrometry. Lc Gc North America, 83-89.

Jones B, Gunneras SA, Petersson SV, Tarkowski P, Graham N, May S, Dolezal K, Sandberg G, Ljung K. 2010. Cytokinin Regulation of Auxin Synthesis in Arabidopsis Involves a Homeostatic Feedback Loop Regulated via Auxin and Cytokinin Signal Transduction. Plant Cell 22, 2956-2969.

Kamada-Nobusada T, Sakakibara H. 2009. Molecular basis for cytokinin biosynthesis. Phytochemistry 70, 444-449.

Kanaujia PK, Pardasani D, Gupta AK, Dubey DK. 2007. Extraction of chemical warfare agents from water with hydrophilic-lipophilic balance and C-18 cartridges: Comparative study. Journal of Chromatography A 1139, 185-190.

Kaneko M, Inukai Y, Ueguchi-Tanaka M, Itoh H, Izawa T, Kobayashi Y, Hattori T, Miyao A, Hirochika H, Ashikari M, Matsuoka M. 2004. Loss-of-function mutations of the rice GAMYB gene impair alpha-amylase expression in aleurone and flower development. Plant Cell 16, 33-44.

Kang J, Yim S, Choi H, Kim A, Lee KP, Lopez-Molina L, Martinoia E, Lee Y. 2015. Abscisic acid transporters cooperate to control seed germination. Nature Communications $\mathbf{6}$.

Kanno Y, Jikumaru Y, Hanada A, Nambara E, Abrams SR, Kamiya Y, Seo M. 2010. Comprehensive Hormone Profiling in Developing Arabidopsis Seeds: Examination of the Site of ABA Biosynthesis, ABA Transport and Hormone Interactions. Plant and Cell Physiology 51, 1988-2001.

Kellermeier F, Chardon F, Amtmann A. 2013. Natural Variation of Arabidopsis Root Architecture Reveals Complementing Adaptive Strategies to Potassium Starvation. Plant Physiology 161, 1421-1432.

Keurentjes JJ, Bentsink L, Alonso-Blanco C, Hanhart CJ, Blankestijn-De Vries H, Effgen S, Vreugdenhil D, Koornneef M. 2007a. Development of a near-isogenic line population of Arabidopsis thaliana and comparison of mapping power with a recombinant inbred line population. Genetics 175, 891-905.

Keurentjes JJ, Fu J, de Vos CH, Lommen A, Hall RD, Bino RJ, van der Plas LH, Jansen RC, Vreugdenhil D, Koornneef M. 2006. The genetics of plant metabolism. Nat Genet 38, 842-849.

Keurentjes JJ, Fu J, Terpstra IR, Garcia JM, van den Ackerveken G, Snoek LB, Peeters AJ, Vreugdenhil D, Koornneef M, Jansen RC. 2007b. Regulatory network construction in Arabidopsis by using genome-wide gene expression quantitative trait loci. Proc Natl Acad Sci U S A 104, 1708-1713.

Keurentjes JJB, Koornneef M, Vreugdenhil D. 2008a. Quantitative genetics in the age of omics. Current Opinion in Plant Biology 11, 123-128. 
Keurentjes JJB, Sulpice R, Gibon Y, Steinhauser MC, Fu JY, Koornneef M, Stitt M, Vreugdenhil D. 2008b. Integrative analyses of genetic variation in enzyme activities of primary carbohydrate metabolism reveal distinct modes of regulation in Arabidopsis thaliana. Genome Biology 9.

Keurentjes JJB, Willems G, van Eeuwijk F, Nordborg M, Koornneef M. 2011. A comparison of population types used for QTL mapping in Arabidopsis thaliana. Plant Genetic Resources-Characterization and Utilization $\mathbf{9}$, 185-188.

Kim S, Plagnol V, Hu TT, Toomajian C, Clark RM, Ossowski S, Ecker JR, Weigel D, Nordborg M. 2007. Recombination and linkage disequilibrium in Arabidopsis thaliana. Nature Genetics 39, 1151-1155.

Kliebenstein DJ. 2009. Advancing Genetic Theory and Application by Metabolic Quantitative Trait Loci Analysis. Plant Cell 21, 1637-1646.

Kliebenstein DJ, Gershenzon J, Mitchell-Olds T. 2001a. Comparative quantitative trait loci mapping of aliphatic, indolic and benzylic glucosinolate production in Arabidopsis thaliana leaves and seeds. Genetics 159, 359-370.

Kliebenstein DJ, Kroymann J, Brown P, Figuth A, Pedersen D, Gershenzon J, Mitchell-Olds T. $2001 \mathrm{~b}$. Genetic control of natural variation in Arabidopsis glucosinolate accumulation. Plant Physiology 126, 811-825.

Ko D, Kang J, Kiba T, Park J, Kojima M, Do J, Kim KY, Kwon M, Endler A, Song WY, Martinoia E, Sakakibara H, Lee Y. 2014. Arabidopsis ABCG14 is essential for the root-to-shoot translocation of cytokinin. Proceedings of the National Academy of Sciences of the United States of America 111, 7150-7155.

Kojima M, Kamada-Nobusada T, Komatsu H, Takei K, Kuroha T, Mizutani M, Ashikari M, UeguchiTanaka M, Matsuoka M, Suzuki K, Sakakibara H. 2009. Highly Sensitive and High-Throughput Analysis of Plant Hormones Using MS-Probe Modification and Liquid ChromatographyTandem Mass Spectrometry: An Application for Hormone Profiling in Oryza sativa. Plant and Cell Physiology 50, 1201-1214.

Kollmer I, Novak O, Strnad M, Schmulling T, Werner T. 2014. Overexpression of the cytosolic cytokinin oxidase/dehydrogenase (CKX7) from Arabidopsis causes specific changes in root growth and xylem differentiation. Plant Journal 78, 359-371.

Konishi T, Takeda T, Miyazaki Y, Ohnishi-Kameyama M, Hayashi T, O'Neill MA, Ishii T. 2007. A plant mutase that interconverts UDP-arabinofuranose and UDP-arabinopyranose. Glycobiology 17, 345-354.

Koornneef M, Alonso-Blanco C, Vreugdenhil D. 2004. Naturally occurring genetic variation in Arabidopsis thaliana. Annual Review of Plant Biology 55, 141-172.

Korstanje R, Paigen B. 2002. From QTL to gene: the harvest begins. Nature Genetics 31, 235-236.

Kover PX, Valdar W, Trakalo J, Scarcelli N, Ehrenreich IM, Purugganan MD, Durrant C, Mott R. 2009. A Multiparent Advanced Generation Inter-Cross to Fine-Map Quantitative Traits in Arabidopsis thaliana. Plos Genetics 5.

Kramer EM, Bennett MJ. 2006. Auxin transport: a field in flux. Trends in Plant Science 11, 382-386.

Kurakawa T, Ueda N, Maekawa M, Kobayashi K, Kojima M, Nagato Y, Sakakibara H, Kyozuka J. 2007. Direct control of shoot meristem activity by a cytokinin-activating enzyme. Nature 445, 652-655.

Kuroha T, Tokunaga H, Kojima M, Ueda N, Ishida T, Nagawa S, Fukuda H, Sugimoto K, Sakakibara H. 2009. Functional analyses of LONELY GUY cytokinin-activating enzymes reveal the importance of the direct activation pathway in Arabidopsis. Plant Cell 21, 3152-3169.

Lacombe B, Achard P. 2016. Long-distance transport of phytohormones through the plant vascular system. Curr Opin Plant Biol 34, 1-8.

Laplaze L, Benkova E, Casimiro I, Maes L, Vanneste S, Swarup R, Weijers D, Calvo V, Parizot B, Herrera-Rodriguez MB, Offringa R, Graham N, Doumas P, Friml J, Bogusz D, Beeckman T, Bennett M. 2007. Cytokinins act directly on lateral root founder cells to inhibit root initiation. Plant Cell 19, 3889-3900.

Larrieu A, Champion A, Legrand J, Lavenus J, Mast D, Brunoud G, Oh J, Guyomarc'h S, Pizot M, Farmer EE, Turnbull C, Vernoux T, Bennett MJ, Laplaze L. 2016. A fluorescent hormone biosensor reveals the dynamics of jasmonate signalling in plants (vol 6, 6043, 2015). Nature Communications 7. 
Lee S, Sergeeva LI, Vreugdenhil D. 2017. Natural variation of hormone levels in Arabidopsis roots and correlations with complex root architecture. J Integr Plant Biol.

Lee S, Sergeeva LI, Vreugdenhil D. 2019. Quantitative trait loci analysis of hormone levels in Arabidopsis roots. PLoS One 14, e0219008.

Leon S. 1998. Ab initio G2(MP2,SVP) study of the reaction of hydroxymethylene with formaldehyde. Chemical Physics Letters 296, 292-298.

Li BH, Gaudinier A, Tang M, Taylor-Teeples M, Nham NT, Ghaffari C, Benson DS, Steinmann M, Gray JA, Brady SM, Kliebenstein DJ. 2014. Promoter-Based Integration in Plant Defense Regulation. Plant Physiology 166, 1803-1820.

Li H, Wang CC, Zhu Q, Du HX, Guan S, Wang FE, Zhang WJ, Fan WJ, Chen ZL, Yang GS, Aboul-Enein HY. 2016. Reduction of matrix effects through a simplified QuEChERS method and using small injection volumes in a LC-MS/MS system for the determination of 28 pesticides in fruits and vegetables. Analytical Methods 8, 5061-5069.

Li HM, Liang H, Li Z, Tang ZX, Fu SL, Geng YY, Yan BJ, Ren ZL. 2015. Dynamic QTL analysis of protein content and glutamine synthetase activity in recombinant inbred wheat lines. Genetics and Molecular Research 14, 8706-8715.

Lisec J, Steinfath M, Meyer RC, Selbig J, Melchinger AE, Willmitzer L, Altmann T. 2009. Identification of heterotic metabolite QTL in Arabidopsis thaliana RIL and IL populations. Plant Journal 59, 777-788.

Mackay TFC, Stone EA, Ayroles JF. 2009. The genetics of quantitative traits: challenges and prospects. Nature Reviews Genetics 10, 565-577.

Manolio TA, Collins FS, Cox NJ, Goldstein DB, Hindorff LA, Hunter DJ, McCarthy MI, Ramos EM, Cardon LR, Chakravarti A, Cho JH, Guttmacher AE, Kong A, Kruglyak L, Mardis E, Rotimi CN, Slatkin M, Valle D, Whittemore AS, Boehnke M, Clark AG, Eichler EE, Gibson G, Haines JL, Mackay TF, McCarroll SA, Visscher PM. 2009. Finding the missing heritability of complex diseases. Nature 461, 747-753. Marhavy P, Bielach A, Abas L, Abuzeineh A, Duclercq J, Tanaka H, Parezova M, Petrasek J, Friml J, Kleine-Vehn J, Benkova E. 2011. Cytokinin Modulates Endocytic Trafficking of PIN1 Auxin Efflux Carrier to Control Plant Organogenesis. Developmental Cell 21, 796-804.

Marhavy P, Duclercq J, Weller B, Feraru E, Bielach A, Offringa R, Friml J, Schwechheimer C, Murphy A, Benkova E. 2014. Cytokinin Controls Polarity of PIN1-Dependent Auxin Transport during Lateral Root Organogenesis. Current Biology 24, 1031-1037.

Mashiguchi K, Tanaka K, Sakai T, Sugawara S, Kawaide H, Natsume M, Hanada A, Yaeno T, Shirasu K, Yao H, McSteen P, Zhao YD, Hayashi K, Kamiya Y, Kasahara H. 2011. The main auxin biosynthesis pathway in Arabidopsis. Proceedings of the National Academy of Sciences of the United States of America 108, 18512-18517.

Matsuda F, Nakabayashi R, Yang ZG, Okazaki Y, Yonemaru J, Ebana K, Yano M, Saito K. 2015. Metabolome-genome-wide association study dissects genetic architecture for generating natural variation in rice secondary metabolism. Plant Journal 81, 13-23.

McKhann HI, Camilleri C, Berard A, Bataillon T, David JL, Reboud X, Le Corre V, Caloustian C, Gut IG, Brunel D. 2004. Nested core collections maximizing genetic diversity in Arabidopsis thaliana. Plant Journal 38, 193-202.

Merilo E, Jalakas P, Laanemets K, Mohammadi O, Horak H, Kollist H, Brosche M. 2015. Abscisic Acid Transport and Homeostasis in the Context of Stomatal Regulation. Molecular Plant 8, 1321-1333.

Mitchell-Olds T, Schmitt J. 2006. Genetic mechanisms and evolutionary significance of natural variation in Arabidopsis. Nature 441, 947-952.

Miyawaki K, Tarkowski P, Matsumoto-Kitano M, Kato T, Sato S, Tarkowska D, Tabata S, Sandberg G, Kakimoto T. 2006. Roles of Arabidopsis ATP/ADP isopentenyltransferases and tRNA isopentenyltransferases in cytokinin biosynthesis. Proc Natl Acad Sci U S A 103, 16598-16603. 
Monchgesang S, Strehmel N, Schmidt S, Westphal L, Taruttis F, Muller E, Herklotz S, Neumann S, Scheel D. 2016. Natural variation of root exudates in Arabidopsis thaliana-linking metabolomic and genomic data. Scientific Reports 6.

Moubayidin L, Di Mambro R, Sabatini S. 2009. Cytokinin-auxin crosstalk. Trends in Plant Science 14, 557562.

Moubayidin L, Perilli S, Dello Ioio R, Di Mambro R, Costantino P, Sabatini S. 2010. The Rate of Cell Differentiation Controls the Arabidopsis Root Meristem Growth Phase. Current Biology 20, 1138-1142.

Muller B, Sheen J. 2008. Cytokinin and auxin interaction in root stem-cell specification during early embryogenesis. Nature 453, 1094-U1097.

Myles S, Peiffer J, Brown PJ, Ersoz ES, Zhang Z, Costich DE, Buckler ES. 2009. Association mapping: critical considerations shift from genotyping to experimental design. Plant Cell 21, 2194-2202.

Nam YJ, Herman D, Blomme J, Chae E, Kojima M, Coppens F, Storme V, Van Daele T, Dhondt S, Sakakibara H, Weigel D, Inze D, Gonzalez N. 2017. Natural Variation of Molecular and Morphological Gibberellin Responses. Plant Physiology 173, 703-714.

Nemhauser JL, Hong F, Chory J. 2006. Different plant hormones regulate similar processes through largely nonoverlapping transcriptional responses. Cell 126, 467-475.

Nonomura AM, Benson AA, Biel KY. 2011. The Path of Carbon in Photosynthesis. Xxvii. Sugar-Conjugated Plant Growth Regulators Enhance General Productivity. Journal of Plant Nutrition 34, 653-664.

Nordborg M, Borevitz JO, Bergelson J, Berry CC, Chory J, Hagenblad J, Kreitman M, Maloof JN, Noyes T, Oefner PJ, StahI EA, Weigel D. 2002. The extent of linkage disequilibrium in Arabidopsis thaliana. Nature Genetics 30, 190-193.

Nordborg M, Innan H. 2002. Molecular population genetics. Current Opinion in Plant Biology 5, 69-73.

Novak O, Hauserova E, Amakorova P, Dolezal K, Strnad M. 2008. Cytokinin profiling in plant tissues using ultra-performance liquid chromatography-electrospray tandem mass spectrometry. Phytochemistry 69, 22142224.

Novak O, Henykova E, Sairanen I, Kowalczyk M, Pospisil T, Ljung K. 2012. Tissue-specific profiling of the Arabidopsis thaliana auxin metabolome. Plant Journal 72, 523-536.

Novakova M, Motyka V, Dobrev PI, Malbeck J, Gaudinova A, Vankova R. 2005. Diurnal variation of cytokinin, auxin and abscisic acid levels in tobacco leaves. Journal of Experimental Botany 56, 2877-2883.

Ooijen V. 2004. MapQTL. Software for the mapping of quantitative trait loci in experimental populations.

Osugi A, Kojima M, Takebayashi Y, Ueda N, Kiba T, Sakakibara H. 2017. Systemic transport of transzeatin and its precursor have differing roles in Arabidopsis shoots. Nature Plants $\mathbf{3}$.

Pan XQ, Welti R, Wang XM. 2010. Quantitative analysis of major plant hormones in crude plant extracts by high-performance liquid chromatography-mass spectrometry. Nature Protocols 5, 986-992.

Pernisova M, Klima P, Horak J, Valkova M, Malbeck J, Soucek P, Reichman P, Hoyerova K, Dubova J, Friml J, Zazimalova E, Hejatko J. 2009. Cytokinins modulate auxin-induced organogenesis in plants via regulation of the auxin efflux. Proceedings of the National Academy of Sciences of the United States of America 106, 3609-3614.

Petersson SV, Johansson AI, Kowalczyk M, Makoveychuk A, Wang JY, Moritz T, Grebe M, Benfey PN, Sandberg G, Ljung K. 2009. An Auxin Gradient and Maximum in the Arabidopsis Root Apex Shown by HighResolution Cell-Specific Analysis of IAA Distribution and Synthesis. Plant Cell 21, 1659-1668.

Pfalz M, Vogel H, Kroymann J. 2009. The Gene Controlling the Indole Glucosinolate Modifier1 Quantitative Trait Locus Alters Indole Glucosinolate Structures and Aphid Resistance in Arabidopsis. Plant Cell 21, 985-999.

Pilet PE, Saugy M. 1987. Effect on Root-Growth of Endogenous and Applied Iaa and Aba - a Critical Reexamination. Plant Physiology 83, 33-38. 
Powell AE, Paleczny AR, Olechowski H, Emery RJN. 2013. Changes in cytokinin form and concentration in developing kernels correspond with variation in yield among field-grown barley cultivars. Plant Physiology and Biochemistry 64, 33-40.

Price AH. 2006. Believe it or not, QTLs are accurate! Trends in Plant Science 11, 213-216.

Puertolas J, Conesa MR, Ballester C, Dodd IC. 2015. Local root abscisic acid (ABA) accumulation depends on the spatial distribution of soil moisture in potato: implications for ABA signalling under heterogeneous soil drying. Journal of Experimental Botany 66, 2325-2334.

Rahman A, Bannigan A, Sulaman W, Pechter P, Blancaflor EB, Baskin TI. 2007. Auxin, actin and growth of the Arabidopsis thaliana primary root. Plant Journal 50, 514-528.

Ristova D, Rosas U, Krouk G, Ruffel S, Birnbaum KD, Coruzzi GM. 2013. RootScape: A Landmark-Based System for Rapid Screening of Root Architecture in Arabidopsis. Plant Physiology 161, 1086-1096.

Romero RM, Roberts MF, Phillipson JD. 1995. CHORISMATE MUTASE IN MICROORGANISMS AND PLANTS. Phytochemistry 40, 1015-1025.

Routaboul JM, Dubos C, Beck G, Marquis C, Bidzinski P, Loudet O, Lepiniec L. 2012. Metabolite profiling and quantitative genetics of natural variation for flavonoids in Arabidopsis. Journal of Experimental Botany 63, 3749-3764.

Ruzicka K, Simaskova M, Duclercq J, Petrasek J, Zazimalova E, Simon S, Friml J, Van Montagu MCE, Benkova E. 2009. Cytokinin regulates root meristem activity via modulation of the polar auxin transport. Proceedings of the National Academy of Sciences of the United States of America 106, 4284-4289.

Sabatini S, Beis D, Wolkenfelt H, Murfett J, Guilfoyle T, Malamy J, Benfey P, Leyser O, Bechtold N, Weisbeek P, Scheres B. 1999. An auxin-dependent distal organizer of pattern and polarity in the Arabidopsis root. Cell 99, 463-472.

Santner A, Estelle M. 2009. Recent advances and emerging trends in plant hormone signalling. Nature 459, 1071-1078.

Sauvage C, Segura V, Bauchet G, Stevens R, Do PT, Nikoloski Z, Fernie AR, Causse M. 2014. GenomeWide Association in Tomato Reveals 44 Candidate Loci for Fruit Metabolic Traits. Plant Physiology 165, 11201132.

Schafer M, Brutting C, Baldwin IT, Kallenbach M. 2016. High-throughput quantification of more than 100 primary- and secondary-metabolites, and phytohormones by a single solid-phase extraction based sample preparation with analysis by UHPLC-HESI-MS/MS. Plant Methods 12.

Schaller GE, Bishopp A, Kieber JJ. 2015. The Yin-Yang of Hormones: Cytokinin and Auxin Interactions in Plant Development. Plant Cell 27, 44-63.

Schmid M, Davison TS, Henz SR, Pape UJ, Demar M, Vingron M, Scholkopf B, Weigel D, Lohmann JU. 2005. A gene expression map of Arabidopsis thaliana development. Nature Genetics 37, 501-506.

Segarra G, Jauregui O, Casanova E, Trillas I. 2006. Simultaneous quantitative LC-ESI-MS/MS analyses of salicylic acid and jasmonic acid in crude extracts of Cucumis sativus under biotic stress. Phytochemistry 67, 395-401.

Sergeeva LI, Keurentjes JJ, Bentsink L, Vonk J, van der Plas LH, Koornneef M, Vreugdenhil D. 2006. Vacuolar invertase regulates elongation of Arabidopsis thaliana roots as revealed by QTL and mutant analysis. Proc Natl Acad Sci U S A 103, 2994-2999.

Sergeeva LI, Vonk J, Keurentjes JJB, van der Plas LHW, Koornneef M, Vreugdenhil D. 2004. Histochemical analysis reveals organ-specific quantitative trait loci for enzyme activities in Arabidopsis. Plant Physiology 134, 237-245.

Shahidi F, Chavan UD, Bal AK, McKenzie DB. 1999. Chemical composition of beach pea (Lathyrus maritimus L.) plant parts. Food Chemistry 64, 39-44.

Shkolnik-Inbar D, Bar-Zvi D. 2010. ABI4 Mediates Abscisic Acid and Cytokinin Inhibition of Lateral Root Formation by Reducing Polar Auxin Transport in Arabidopsis. Plant Cell 22, 3560-3573. 
Slovak R, GoschI C, Su XX, Shimotani K, Shiina T, Busch W. 2014. A Scalable Open-Source Pipeline for Large-Scale Root Phenotyping of Arabidopsis. Plant Cell 26, 2390-2403.

Smehilova M, Dobruskova J, Novak O, Takac T, Galuszka P. 2016. Cytokinin-Specific Glycosyltransferases Possess Different Roles in Cytokinin Homeostasis Maintenance. Frontiers in Plant Science $\mathbf{7}$.

Smith S, De Smet I. 2012. Root system architecture: insights from Arabidopsis and cereal crops Introduction. Philosophical Transactions of the Royal Society B-Biological Sciences 367, 1441-1452.

Sofo A, Vitti A, Nuzzaci M, Tataranni G, Scopa A, Vangronsveld J, Remans T, Falasca G, Altamura MM, Degola F, di Toppi LS. 2013. Correlation between hormonal homeostasis and morphogenic responses in Arabidopsis thaliana seedlings growing in a $\mathrm{Cd} / \mathrm{Cu} / \mathrm{Zn}$ multi-pollution context. Physiologia Plantarum 149, 487498.

Song XL, Zhang TZ. 2010. Molecular mapping of quantitative trait loci controlling chlorophyll content at different developmental stages in tetraploid cotton. Plant Breeding 129, 533-540.

Spiess GM, Hausman A, Yu P, Cohen JD, Rampey RA, Zolman BK. 2014. Auxin Input Pathway Disruptions Are Mitigated by Changes in Auxin Biosynthetic Gene Expression in Arabidopsis. Plant Physiology 165, 10921104.

Stahnke H, Kittlaus S, Kempe G, Alder L. 2012. Reduction of Matrix Effects in Liquid ChromatographyElectrospray Ionization-Mass Spectrometry by Dilution of the Sample Extracts: How Much Dilution is Needed? Analytical Chemistry 84, 1474-1482.

Stirnberg P, Ward S, Leyser O. 2010. Auxin and strigolactones in shoot branching: intimately connected? Biochemical Society Transactions 38, 717-722.

Suhre K, Shin SY, Petersen AK, Mohney RP, Meredith D, Wagele B, Altmaier E, Deloukas P, Erdmann J, Grundberg E, Hammond CJ, de Angelis MH, Kastenmuller G, Kottgen A, Kronenberg F, Mangino M, Meisinger C, Meitinger T, Mewes HW, Milburn MV, Prehn C, Raffler J, Ried JS, Romisch-Margl W, Samani NJ, Small KS, Wichmann HE, Zhai GJ, Illig T, Spector TD, Adamski J, Soranzo N, Gieger C, CardioGram. 2011. Human metabolic individuality in biomedical and pharmaceutical research. Nature 477, 54-U60.

Sutka M, Li GW, Boudet J, Boursiac Y, Doumas P, Maurel C. 2011. Natural Variation of Root Hydraulics in Arabidopsis Grown in Normal and Salt-Stressed Conditions. Plant Physiology 155, 1264-1276.

Svacinova J, Novak O, Plackova L, Lenobel R, Holik J, Strnad M, Dolezal K. 2012. A new approach for cytokinin isolation from Arabidopsis tissues using miniaturized purification: pipette tip solid-phase extraction. Plant Methods 8, 17.

Swarup R, Parry G, Graham N, Allen T, Bennett M. 2002. Auxin cross-talk: integration of signalling pathways to control plant development. Plant Molecular Biology 49, 411-426.

Swarup R, Perry P, Hagenbeek D, Van Der Straeten D, Beemster GTS, Sandberg G, Bhalerao R, Ljung K, Bennett MJ. 2007. Ethylene upregulates auxin biosynthesis in Arabidopsis seedlings to enhance inhibition of root cell elongation. Plant Cell 19, 2186-2196.

Takehisa H, Sato Y, Igarashi M, Abiko T, Antonio BA, Kamatsuki K, Minami H, Namiki N, Inukai Y, Nakazono M, Nagamura Y. 2012. Genome-wide transcriptome dissection of the rice root system: implications for developmental and physiological functions. Plant Journal 69, 126-140.

Tanaka K, Hayashi K, Natsume M, Kamiya Y, Sakakibara H, Kawaide H, Kasahara H. 2014. UGT74D1 Catalyzes the Glucosylation of 2-Oxindole-3-Acetic Acid in the Auxin Metabolic Pathway in Arabidopsis. Plant and Cell Physiology 55, 218-228.

Tanimoto E. 2005. Regulation of root growth by plant hormones - Roles for auxin and gibberellin. Critical Reviews in Plant Sciences 24, 249-265.

Tarkowska D, Novak O, Flokova K, Tarkowski P, Tureckova V, Gruz J, Rolcik J, Strnad M. 2014. Quo vadis plant hormone analysis? Planta 240, 55-76. 
Taylor JLS, vanStaden J. 1997. Variation in the level and type of cytokinin with the stage of root development in Impatiens wallerana Hook. f. stem cuttings. Plant Growth Regulation 22, 175-180.

Taylor PJ. 2005. Matrix effects: The Achilles heel of quantitative high-performance liquid chromatographyelectrospray-tandem mass spectrometry. Clinical Biochemistry 38, 328-334.

Teale WD, Ditengou FA, Dovzhenko AD, Li X, Molendijk AM, Ruperti B, Paponov I, Palme K. 2008. Auxin as a model for the integration of hormonal signal processing and transduction. Molecular Plant 1, 229237.

Tian HY, De Smet I, Ding ZJ. 2014. Shaping a root system: regulating lateral versus primary root growth. Trends in Plant Science 19, 426-431.

Tine Y, Diop A, Diatta W, Desjobert JM, Boye CSB, Costa J, Wele A, Paolini J. 2017. Chemical Diversity and Antimicrobial Activity of Volatile Compounds from Zanthoxylum zanthoxyloides Lam. according to Compound Classes, Plant Organs and Senegalese Sample Locations. Chemistry \& Biodiversity 14.

Todesco M, Balasubramanian S, Hu TT, Traw MB, Horton M, Epple P, Kuhns C, Sureshkumar S, Schwartz C, Lanz C, Laitinen RAE, Huang Y, Chory J, Lipka V, Borevitz JO, Dangl JL, Bergelson J, Nordborg M, Weigel D. 2010. Natural allelic variation underlying a major fitness trade-off in Arabidopsis thaliana. Nature 465, 632-U129.

Toubiana D, Semel Y, Tohge T, Beleggia R, Cattivelli L, Rosental L, Nikoloski Z, Zamir D, Fernie AR, Fait A. 2012. Metabolic Profiling of a Mapping Population Exposes New Insights in the Regulation of Seed Metabolism and Seed, Fruit, and Plant Relations. Plos Genetics 8.

Tuberosa R, Sanguineti MC, Landi P, Salvi S, Casarini E, Conti S. 1998. RFLP mapping of quantitative trait loci controlling abscisic acid concentration in leaves of drought-stressed maize (Zea mays L.). Theoretical and Applied Genetics 97, 744-755.

Unterholzner SJ, Rozhon W, Papacek M, Ciomas J, Lange T, Kugler KG, Mayer KF, Sieberer T, Poppenberger B. 2015. Brassinosteroids Are Master Regulators of Gibberellin Biosynthesis in Arabidopsis. Plant Cell 27, 2261-2272.

Van Eeckhaut A, Lanckmans K, Sarre S, Smolders I, Michotte Y. 2009. Validation of bioanalytical LCMS/MS assays: evaluation of matrix effects. J Chromatogr B Analyt Technol Biomed Life Sci 877, 2198-2207.

Vanstraelen M, Benkova E. 2012. Hormonal interactions in the regulation of plant development. Annu Rev Cell Dev Biol 28, 463-487.

Verpoorte R. 1998. Exploration of nature's chemodiversity: the role of secondary metabolites as leads in drug development. Drug Discovery Today 3, 232-238.

Vreugdenhil D, Aarts MGM, Koornneef M, Nelissen H, Ernst WHO. 2004. Natural variation and QTL analysis for cationic mineral content in seeds of Arabidopsis thaliana. Plant Cell and Environment 27, 828-839.

Wajs-Bonikowska A, Stojakowska A, Kalemba D. 2012. Chemical Composition of Essential Oils from a Multiple Shoot Culture of Telekia speciosa and Different Plant Organs. Natural Product Communications 7, 625628.

Wang J, Ma XM, Kojima M, Sakakibara H, Hou BK. 2011. N-Glucosyltransferase UGT76C2 is Involved in Cytokinin Homeostasis and Cytokinin Response in Arabidopsis thaliana. Plant and Cell Physiology 52, 22002213.

Wang L, Xie WB, Chen Y, Tang WJ, Yang JY, Ye RJ, Liu L, Lin YJ, Xu CG, Xiao JH, Zhang QF. 2010. A dynamic gene expression atlas covering the entire life cycle of rice. Plant Journal 61, 752-766.

Wang XD, Wang H, Long Y, Liu LZ, Zhao YJ, Tian JH, Zhao WG, Li BJ, Chen L, Chao HB, Li MT. 2015. Dynamic and comparative QTL analysis for plant height in different developmental stages of Brassica napus $L$. Theoretical and Applied Genetics 128, 1175-1192.

Weigel D. 2012. Natural variation in Arabidopsis: from molecular genetics to ecological genomics. Plant Physiol 158, 2-22.

Weigel D, Mott R. 2009. The 1001 Genomes Project for Arabidopsis thaliana. Genome Biology 10. 
Weng JK. 2014. The evolutionary paths towards complexity: a metabolic perspective. New Phytologist 201, 1141-1149.

Weng YQ, Colle M, Wang YH, Yang LM, Rubinstein M, Sherman A, Ophir R, Grumet R. 2015. QTL mapping in multiple populations and development stages reveals dynamic quantitative trait loci for fruit size in cucumbers of different market classes. Theoretical and Applied Genetics 128, 1747-1763.

Went FW, Thimann KV. 1937. Phytohormones. Macmillan, New York.

Werner T, Schmulling T. 2009. Cytokinin action in plant development. Curr Opin Plant Biol 12, 527-538.

Wilkinson S, Davies WJ. 2010. Drought, ozone, ABA and ethylene: new insights from cell to plant to community. Plant Cell and Environment 33, 510-525.

Wilkinson S, Kudoyarova GR, Veselov DS, Arkhipova TN, Davies WJ. 2012. Plant hormone interactions: innovative targets for crop breeding and management. Journal of Experimental Botany 63, 3499-3509.

Winter D, Vinegar B, Nahal H, Ammar R, Wilson GV, Provart NJ. 2007. An "Electronic Fluorescent Pictograph" Browser for Exploring and Analyzing Large-Scale Biological Data Sets. Plos One 2.

Wurschum T, Liu WX, Busemeyer L, Tucker MR, Reif JC, Weissmann EA, Hahn V, Ruckelshausen A, Maurer HP. 2014. Mapping dynamic QTL for plant height in triticale. Bmc Genetics 15, 8.

Xing LB, Zhang D, Li YM, Shen YW, Zhao CP, Ma JJ, An N, Han MY. 2015. Transcription Profiles Reveal Sugar and Hormone Signaling Pathways Mediating Flower Induction in Apple (Malus domestica Borkh.). Plant and Cell Physiology 56, 2052-2068.

Yamaguchi N, Winter CM, Wu MF, Kanno Y, Yamaguchi A, Seo M, Wagner D. 2014. Gibberellin Acts Positively Then Negatively to Control Onset of Flower Formation in Arabidopsis. Science 344, 638-641.

Yu JM, Holland JB, McMullen MD, Buckler ES. 2008. Genetic design and statistical power of nested association mapping in maize. Genetics 178, 539-551.

Zhang JH, Tardieu F. 1996. Relative contribution of apices and mature tissues to ABA synthesis in droughted maize root systems. Plant and Cell Physiology 37, 598-605.

Zhang KW, Novak OR, Wei ZY, Gou MY, Zhang XB, Yu Y, Yang HJ, Cai YH, Strnad M, Liu CJ. 2014. Arabidopsis ABCG14 protein controls the acropetal translocation of root-synthesized cytokinins. Nature Communications $\mathbf{5}$.

Zhang ZY, Ober JA, Kliebenstein DJ. 2006. The gene controlling the quantitative trait locus EPITHIOSPECIFIER MODIFIER1 alters glucosinolate hydrolysis and insect resistance in Arabidopsis. Plant Cell 18, 1524-1536.

Zhao ZG, Zhang YH, Liu X, Zhang X, Liu SC, Yu XW, Ren YL, Zheng XM, Zhou KN, Jiang L, Guo XP, Gai Y, Wu CY, Zhai HQ, Wang HY, Wan JM. 2013. A Role for a Dioxygenase in Auxin Metabolism and Reproductive Development in Rice. Developmental Cell 27, 113-122.

Zhao ZX, Assmann SM. 2011. The glycolytic enzyme, phosphoglycerate mutase, has critical roles in stomatal movement, vegetative growth, and pollen production in Arabidopsis thaliana. Journal of Experimental Botany 62, 5179-5189.

Zhu JM, Ingram PA, Benfey PN, Elich T. 2011. From lab to field, new approaches to phenotyping root system architecture. Current Opinion in Plant Biology 14, 310-317. 


\section{Summary}

Plants synthesize a myriad of metabolites, far more than those produced by most other organisms. Plant hormones are a unique set of compounds, with distinctive metabolism and properties that affect physiological processes during development and growth. Their underlying genetic variation will have attracted much research attention by virtue of junctions of NGS technologies and metabolomics.

The aim of this thesis was to study natural variation of plant hormones in Arabidopsis. To obtain reliable quantitative data of levels of hormone using LC-ESI-MS, I first developed a set of methodological processes related to extraction and purification. A set of Arabidopsis ecotypes was chosen, and studied to earn various aspects of natural variation with traits of mature roots. Based on results of these works, my study has moved to classical linkage mapping analysis to find genetic loci responsible for levels of a few sets of hormone in roots and leaves of Arabidopsis.

Chapter 2 describes the problem of matrix effects caused by impurities in semi-purified extracts, on the accuracy of data derived from LC-ESI-mass spectrometry. Matrix effects may result in both ion suppression and enhancement, and severely affects quantitative data of hormone levels. Without proper ways to minimize matrix effects, hormone data would be unreliable, and would not allow accurate QTL mapping. I validated a few key-points that are critical for determining the levels of a wide range of plant hormones in Arabidopsis extracts based on a one-step solid phase extraction (SPE) method. For the simplified purification of Arabidopsis extracts, a commercially available HLB sorbent was chosen to recover a wide range of chemically diverse series of hormone analytes. Dilution using a much smaller starting sample (e.g., $2.5 \mathrm{mg}$ ) reduced the matrix effects considerably but additional measures were required for most of the analytes. Flushing the HLB-SPE column with acidic methanol was more effective to reduce matrix effect than acetonitrile based eluent. At the end, I proposed a series of steps and procedures to optimize the protocol for hormone analysis in LC-ESIMS.

Chapter 3 describes natural variation of hormone levels found in 13 Arabidopsis ecotypes. Variations of hormone level among the accessions were remarkably small, viz., less than three-fold difference between extremes. For CKs, relatively larger variations were found for ribosides and glucosides, as compared to the free bases. Root phenotypic traits of these accessions were also measured, using a new parameter (mature root unit) for complex root systems, and correlation analyses were done between hormone data and mature root traits. For root phenotyping, length-related traits-lateral root length and total root length-showed larger variations than lateral root numberrelated ones. Antagonistic interactions between hormones (IAA and trans-zeatin) were detected for root weight. These findings provide enough basis to warrant a quantitative genetic analysis in plant 
Chapter 4 reports diverse QTLs that are responsible for hormone levels of CKs, SA and JA in roots of Arabidopsis Ler $\times$ Cvi RIL population. QTL analysis of two sub-populations, viz., vegetative and flowering plants revealed that many of the QTLs were development-specific, suggesting that the transition to flowering has a profound effect on hormone metabolism. Using near-isogenic lines, several significant QTLs were confirmed; three co-localized QTL regions were responsible for determining several CK metabolites. Using a knock-out plant, a functional role of zeatin Nglucosyltransferase gene (UGT76C2) underlying a large-effect QTL for levels of tZ N-glucosides and tZRMP was evaluated in CK metabolism. Pleotropic effects of this gene were found for levels of CK in both roots and leaves, but significant changes of morphological traits were observed only in roots. This suggests that $\mathrm{CK} N$-glucosides play an important role in root development. I also advocated the possibility of genetic regulation of concentration ratio between tZ7G and tZ9G based on a newly observed QTL of the trait.

As a further step of QTL analysis after Chapter 4, the study was extended to leaves. Chapter 5 describes the analysis of 5 groups of hormones in rosette leaves of the same RIL population. QTL analysis showed a multitude of significant loci for levels of IAA, ABAs and CKs. Also for leaves, development-specific QTLs were detected in two sub-populations, vegetative and flowering lines. QTLs for ratios between hormone metabolites belonging to the same group but also to different groups were found and some of them partly co-localized with those of single compounds, implying that QTLs for single hormones may also affect the balance between hormones. The detection of QTLs for ratios between structurally unrelated hormones (e.g. auxin : ABA) and further fine mapping may help unravelling genetic elements underlying hormone interactions in the regulation of plant development and stress responses.

Finally, in Chapter 6, several issues arising from the separate experiments are taken into consideration. The main significance of this $\mathrm{PhD}$ thesis is the experimental confirmation that finding QTLs for hormone metabolism is feasible and worth being extended to other populations of Arabidopsis and to crop plants. I anticipate that in the near future metabolomics study towards natural variation of plant hormones will be part of interesting theme in quantitative genetics. It will provide us to gain a better understanding of the complexity of molecular mechanism underlying hormone metabolism in plants. 


\section{Acknowledgements}

My PhD journey started on 18 January 2012 in WUR. I remember the moment and scene through pictures taken the early morning of the day when we (Gyujin, Euijin and Chongiee) first arrived at Schiphol Airport in a little bit of tense mood ourselves. I thank Dr. Jiwoon Lee (RDA, South Korea) for coming to the airport with a van to bring us to Wageningen. He gave me a series of help especially, including his own car as emergency ambulance under "colicky pain" due to urinary stones. This became the opportunity I could experience the Dutch hospital. I also thank medical staff of Gelderse Vallei Hospital who provided safe treatments to me and my family.

I would present my special gratitude and respect to my supervisor, Dick who has taken care of every step of my $\mathrm{PhD}$ course for years, even after his retirement. I may not be able to reach this stage of graduation if he didn't have long-standing patience on me and provide continuous supports to my works. My special thanks to daily supervisor, Lidiya who had cared of my works in the lab and around Plant Physiology Group. I acknowledge my promotor, Harro who gave me cordial greetings and talks often coming to my desk. He was also the first contact scholar and benefactor who gave me an opportunity to become $\mathrm{PhD}$ candidate in the Netherlands. I thank to teaching staff in the Lab of Plant Physiology, Christa, Sander, Leonie, Wilco, Robert and Henk who provided precious advices in $\mathrm{PhD}$ meetings, literature discussion and various talks. I also would like to mention technical supports and helps given by Francel, Bas, Iris, Richard, Leo, Marielle, Rina, Diaan, Karen and Juriaan.

My warm thanks to PhD students: Bing, Yanxia, Yanting, Jun, Umidjon, Yuanyuan, Mark, Nasr, Johanna, Xi, Beatriz, Mariafe, Arman, Margriet, Gonda, Esmer, Emilie, Elise, Wei, Bo, Nafiseh, Natalia, Melissa, Giovanni, Alexandre, Marielle. I am grateful to Korean students who shared memorable activities in Wageningen and in the Netherlands, especially to 현권, 재윤, 세종, 제승.

I want to express special gratitude to the Korean Church (사랑의교회) members in Eindhoven, who gave us strength and comfort. For this, I would like to present special thanks to Pastor, 권준 and his family. Almost every weekend for 4.5 years, we could have been able to attend worship service due to Toyota Aygo, a tiny but reliable to drive. We thank the church members for occasional invitations to dinner and talks after the service. My family could have friendship with a few Korean ladies who married Dutch guys and lived around in Ede, Bennekom and Veenendaal. I appreciate their contacts and gatherings for Chongiee, especially special thanks to Mee-ae

My respect to Tim Vos, a veteran, in Wageningen who was one of 5,322 Dutch participants in the Korean War. He was the special elder who reminded me of desperate and miserable historic time in South Korea. I appreciate his dedication and affection toward my country. 
I acknowledge Dutch teachers who taught and cared my daughters in Wageningen and Bennekom, in particular to teachers in charge of their classes and Mr Driessen (principal in those days) in EbenHaezerschool. I also thank to Dutch parents of daughter's friends who greeted and presented us in hospitality on the way of going to school.

I would present my appreciation to colleagues of Organic Agriculture Research Institute in GBARES who would compensate my long absence with their frequent weekend/holiday-duty with increased work-force.

In memoir, my love to father, 이태희 and mother-in-law, 김금지 who I couldn't see his and her last breaths in this world. I appreciate enormous care, love and prayer of my siblings: my brothers, 이상욱, 이상탁; sister, 이순영; sister-in-laws, 김은숙, 김순영; brother-in-law, 김성진; nephews and nieces, 효인,정호, 보형, 예진, 지형(승윤), 유경. I am also very grateful to Chongiee's family: father-in-law, 조차근; sister-in-laws, 조선주, 조효주; brother-in-laws, 조철희, 조용세; and their families. My special thanks to 서문영 who visited us twice while living in the Netherlands. My love to daughters, 규진, 의진 who unexpectedly had to learn new language and study in the Netherlands. Both of you had been the essence of my comfort as you've been today and further. Finally, I would like to present my love and honour to wife, 조청이 who have trusted and supported me all the days of my life. 


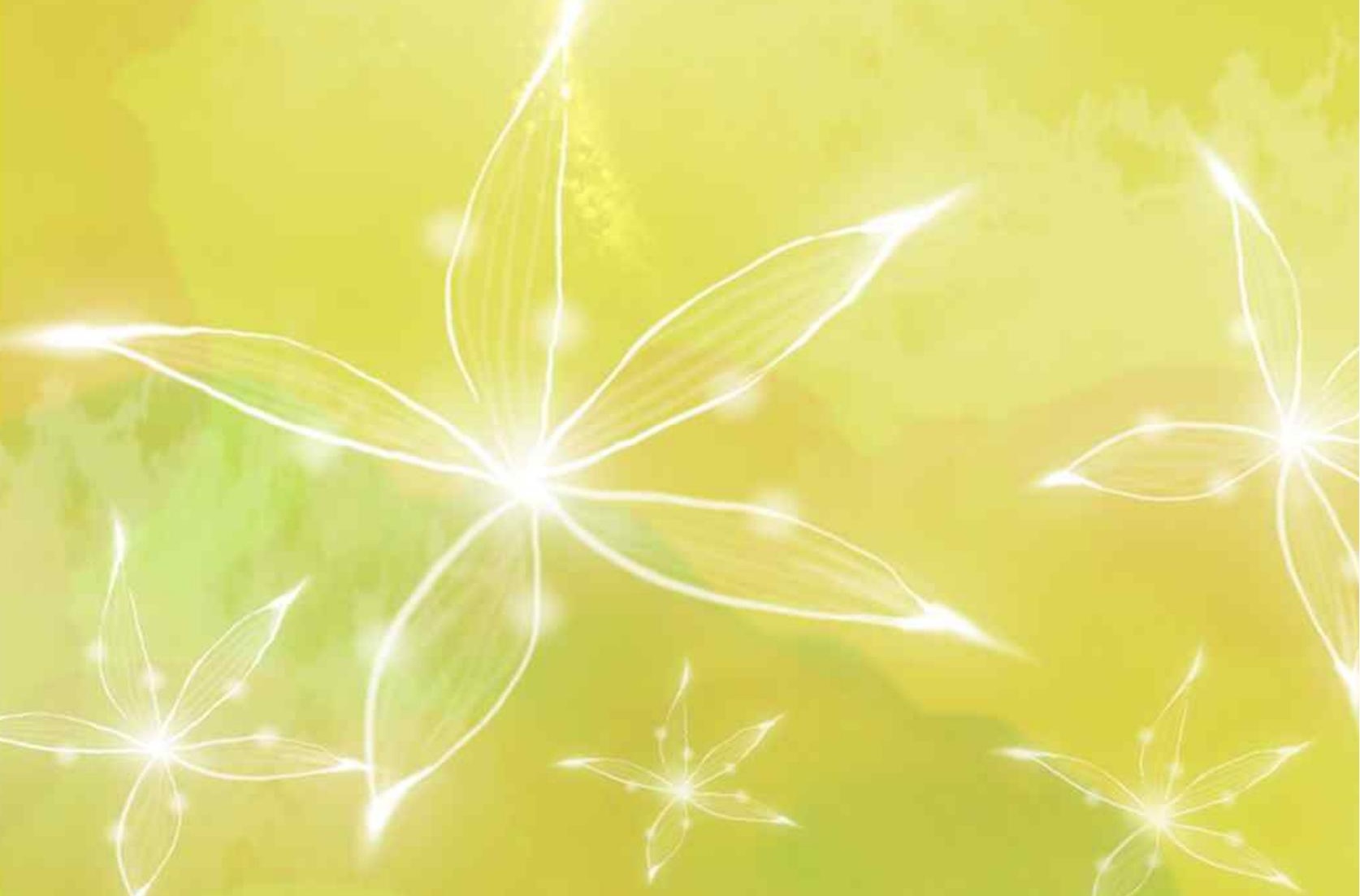

